\title{
Infrared Spectra of High Polymers
}

\author{
By \\ S. KRIMM \\ Harrison M. Randall Laboratory of Physics \\ The University of Michigan, Ann Arbor, Mich.
}

With 22 Figures

Table of Contents

I. Introduction. . . . . . . . . . . . . . . . 52

A. Origin of Vibrational Spectra . . . . . . . . . . . . . 52

B. Group Frequencies . . . . . . . . . . . . . . . . . 58

II. Some Experimental Techniques . . . . . . . . . . . . . . . . . . 70

A. Far Infrared Spectroscopy . . . . . . . . . . . . 70

B. Polarization Measurements . . . . . . . . . . . . . . . . . . 71

C. Specimen Preparation . . . . . . . . . . . . . . . 75

III. Methods of Spectral Analysis of High Polymers . . . . . . . . . . . 78

A. Theoretical Methods of Analysis. . . . . . . . . . . . . . . 78

1. Symmetry Analysis . . . . . . . . . . . . . . . . 78

2. Normal Vibration Analysis. . . . . . . . . . . . . 87

B. Experimental Methods of Analysis . . . . . . . . . . . . 90

1. Far Infrared and Raman Spectra . . . . . . . . . . . 90

2. Deuteration Studies. . . . . . . . . . . . . . 91

3. Polarized Radiation Studies . . . . . . . . . . . . . 95

4. Specimen Variation . . . . . . . . . . . . . . 100

IV. Spectra of High Polymers . . . . . . . . . . . . . . 102

A. Polyethylene . . . . . . . . . . . . . . 103

B. Polytetrafluoroethylene . . . . . . . . . . . . 115

C. Polyvinyl Chloride . . . . . . . . . . . 120

D. Polyvinyl Alcohol . . . . . . . . . . . . . . . . . . . 127

E. Polypropylene . . . . . . . . . . . . . . . 135

F. Polystyrene . . . . . . . . . . . . . . . 140

G. Polyvinylidene Chloride . . . . . . . . . . . . . . 147

H. Polyethylene Terephthalate . . . . . . . . . . . . . 153

V. Conclusion .................. . 160

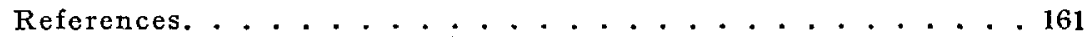




\section{Introduction}

The study of the vibrational and rotational spectra of small molecules has provided a powerful method for probing certain aspects of molecular structure and intramolecular forces. In great part this has resulted from the highly developed theoretical tools which have been available and from the feasibility of applying these tools to the detailed analysis of such molecules [Herzberg (76); Wilson, Decius, Cross (245)]. A comparable analysis of the spectra of large molecules is far from having been achieved. Although the basic principles underlying the origin of the spectra of large molecules are understood, the complexity of the problem has hindered the achievement of the kind of detailed solution which is possible for small molecules. As a result it has been observed that " . . . the most that can be stated at present about a large organic molecule or polymer is that it does or does not contain certain chemical groups, and even this statement has frequently to be hedged about with qualifications" [SUTHERLAND (213)]. The implication of this situation is that if a complete analysis of the spectrum of a large molecule could be achieved, additional significant information would undoubtedly be a consequence. In recent years increasing efforts have been made in this direction, particularly in the case of high polymers. This has been a result of the exploitation of certain simplifying features of polymer spectra, the development of techniques which have extended and amplified our experimental data, and the realization that infrared spectroscopy provides a structural tool which often complements other methods such as x-ray diffraction [SutherLAND (213); KrIMm (96)]. The aims of this article are to discuss the kind of approach to the complete analysis of high polymer spectra which is developing at the present time, and to examine the results of its application to the spectra of several high polymers. We will begin by reviewing briefly the theory of the origin of vibrational spectra and by indicating how this theory was initially applied to the spectroscopy of complex molecules.

\section{A. Origin of Vibrational Spectra}

The energy of a molecule is determined by several factors: the state of motion (in the classical sense) of its electrons, the vibrations of its nuclei, the rotation of the molecule as a unit, and its translation in space. The translation of a molecule does not lead to any interaction with electromagnetic radiation, and we will therefore neglect it in this discussion. The rotation of a molecule can give rise to the absorption or Raman scattering of radiation, but since, under the usual conditions in which high polymers are examined, such free rotation of the entire molecule is not feasible, we will leave out of consideration this contribution to the energy. The energy of such a non-rotating molecule is therefore 
determined by electronic and vibrational contributions. In general, it would not be possible a priori to separate these two, i. e., to treat the vibrational motion of the nuclei as taking place in a constant force field (determined by the electronic configuration). Were this so, the problem would be a quite complex one, comparable to discussing the motion of masses connected by springs whose force constants changed during the vibrational motion. However, to a very good approximation, the total energy of a molecule can be considered as the sum of an electronic energy (due solely to the state of motion of the electrons) and a vibrational energy (due solely to the vibration of the nuclei). This arises because (speaking classically), although the electrons and the nuclei are subjected to comparable (electrical) forces, the mass of the former is several orders of magnitude smaller than that of the latter, and the electronic motions are therefore of much higher frequency than the nuclear oscillations. Since these oscillations are also of very small amplitude compared to interatomic distances, the nuclei vibrate essentially in an averaged electron distribution. Another way of stating this is to say that when two oscillatory systems of widely different frequencies are coupled together, the interaction between them leaves the original frequencies essentially unaltered.

The interaction of such a system with an electromagnetic field is of course determined by the well-known quantum conditions. The total energy of the molecule (considered here to consist of electronic plus vibrational energies) cannot assume any of a continuous range of values but only certain discrete (quantized) values. An absorption band will result when the energy of the molecule is changed from one discrete value to a higher one by the absorption of a quantum of radiation. The frequency of such a band, in $\mathrm{cm}^{-1}$, is given by the Bohr relation

$$
\omega \equiv \frac{10^{4}}{\lambda}=\frac{E^{\prime}-E^{\prime \prime}}{h c}
$$

where $\lambda$ is the wavelength in microns, $E^{\prime}$ and $E^{\prime \prime}$ the energies, in ergs, of the upper and lower states respectively, $h$ is Planck's constant, and $c$ is the velocity of light. In order to change the electronic energy from its ground state value, radiation quanta in the visible and ultraviolet regions must be absorbed by the molecule. Absorption of the lower energy quanta of the infrared region (roughly from $10 \mathrm{~cm}^{-1}$ to $12,500 \mathrm{~cm}^{-1}$ ) results in changes only in the vibrational energy of the molecule while it is in its ground electronic state, and it is with such vibrational spectra that we are concerned. The basic problem of vibrational spectroscopy is to correlate the experimentally observable energy levels with the structure and force function of the molecule.

The solution of this problem must of course be approached quantum mechanically. When this is done, however, it can be shown that, in the 
harmonic oscillator approximation, it is reducible to a classical analysis of the vibrating system. This comes about as follows: when the Schrödinger wave equation for a system of $N$ particles is set up in order to obtain the vibrational energy levels, it is found [PAULING and WILson (165)] that this equation is separable and readily soluble if it is written in terms of so-called normal coordinates rather than Cartesian coordinates. The normal coordinates describe modes of oscillation (normal modes) having the characteristic that in such a mode all nuclei in the molecule move (generally in phase) with simple harmonic motion of the same frequency. The result of using normal coordinates is that the total energy can be expressed as the sum of the energies associated with the individual normal modes, each quantized according to the relation

$$
E_{i}=h v_{i}\left(v_{i}+\frac{1}{2}\right) \quad v_{i}=0,1,2, \ldots
$$

where $\nu_{i}$ is the classical oscillation frequency for the normal vibration $i$, and $v_{i}$ is the vibrational quantum number. The problem is therefore reduced to determining the classical vibration frequencies associated with the normal modes of oscillation. This also constitutes the classical solution of the vibrational problem, because, since the normal modes are the only simple periodic vibrations of the molecule, the normal frequencies will be the ones absorbed by the molecule in its interaction with the periodically varying electric field of the incident radiation.

The determination of these normal frequencies, and the forms of the normal vibrations, thus becomes the primary problem in correlating the structure and internal forces of the molecule with the observed vibrational spectrum. It is the complexity of this problem for large molecules which has hindered the kind of detailed solution that can be achieved with small molecules. In the general case, a solution of the equations of motion in normal coordinates is required. Let the Cartesian displacement coordinates of the $N$ nuclei of a molecule be designated by $q_{1}, q_{2}, \ldots q_{3 N}$. The potential energy of the oscillating system is not accurately known in the absence of a solution to the quantum mechanical problem of the electronic energies, but for small displacements it can be quite well approximated by a power series expansion in the displacements:

$$
V=\frac{1}{2}\left[V_{0}+\sum_{i=1}^{3 N}\left(\frac{\partial V}{\partial q_{i}}\right)_{0} q_{i}+\sum_{i, j=1}^{3 N}\left(\frac{\partial^{2} V}{\partial q_{i} \partial q_{j}}\right)_{0} q_{i} q_{j}+\cdots\right]
$$

By choosing the equilibrium position as the zero energy, $V_{0}$ can be set equal to zero. Also, since the cnergy of the molecule is a minimum at the equilibrium position, the $\left(\frac{\partial V}{\partial q_{i}}\right)_{0}$ are all zero. Therefore, if we neglect higher order terms,

$$
V=\frac{1}{2} \sum_{i, j=1}^{3 N}\left(\frac{\partial^{2} V}{\partial q_{i} \partial q_{j}}\right)_{0} q_{i} q_{j}=\frac{1}{2} \sum_{i, j=1}^{3 N} k_{i j} q_{i} q_{j}
$$


where the $k_{i j}$ are known as force constants. The kinetic energy of the system is given by

$$
T=\frac{1}{2} \sum_{i=1}^{3 N} m_{i} \dot{q}_{i}^{2}
$$

where $m_{i}$ is the mass of nucleus $i$. By analogy with equation (4) this can be written

where in this case

$$
T=\frac{1}{2} \sum_{i, j=1}^{3 N} b_{i j} \dot{q}_{i} \dot{q}_{j}
$$

$$
b_{i j}=0 \text { for } i \neq j, \quad b_{11}=b_{22}=b_{33}=m_{1}, \quad b_{44}=b_{55}=b_{66}=m_{2}, \ldots
$$

The normal coordinates are required to be combinations of the $q_{i}$ such that the total energy, when expressed in terms of these coordinates, becomes the sum of the energies of individual harmonic oscillators. This means that no cross terms should appear in $V$ and $T$ when written in normal coordinates. For an oscillator of mass 1 , we would therefore have

and

$$
V=\frac{1}{2} \sum_{i=1}^{3 N} \lambda_{i} Q_{i}^{2}
$$

$$
T=\frac{1}{2} \sum_{i=1}^{3 N} \dot{Q}_{i}^{2}
$$

where the $Q_{i}$ are the normal coordinates, and $\lambda_{i}=4 \pi^{2} \nu_{i}^{2}$. Equations (8) and (9) are obtained from (4) and (6) by a substitution of the form

$$
q_{k}=\sum_{i=1}^{3 N} c_{k i} Q_{i} \quad k=1,2, \ldots 3 N
$$

This transformation will exist if there is a linear transformation expressing the $Q$ 's in terms of the $q$ 's, and equations (8) and (9) will result by suitably choosing the coefficients $c_{k i}$. By introducing equations (8) and (9) into the Lagrangian form of the equations of motion, viz.,

$$
\frac{d}{d t}\left(\frac{\partial T}{\partial \dot{Q}_{l}}\right)+\frac{\partial V}{\partial Q_{l}}=0 \quad l=1,2, \ldots 3 N
$$

we obtain for the equations of motion

$$
Q_{l}+\lambda_{l} Q_{l}=0 \quad l=1,2, \ldots 3 N
$$

The solutions of these equations are

$$
Q_{l}=Q_{l}^{0} \sin \left(\sqrt{\lambda_{l}} t+\varphi_{l}\right) \quad l=1,2, \ldots 3 N
$$

where the $Q_{2}^{0}$ are amplitude constants. From equation (10) we see, therefore, that in a given normal mode, $Q_{l}$, all of the nuclei oscillate with the same frequency; that is

$$
\begin{aligned}
q_{k} & =c_{k l} Q_{l}^{0} \sin \left(\sqrt{\lambda_{l}} t+\varphi_{l}\right) \quad k=1,2, \ldots 3 N \\
& =A_{k} \quad \sin \left(\sqrt{\lambda_{l}} t+\varphi_{l}\right)
\end{aligned}
$$


In order to determine the normal frequencies, the general form of equation (14) is introduced into the Lagrangian equations of motion in terms of the $q$ 's. The result is

$$
-b_{i i} \lambda A_{i}+\sum_{j=1}^{3 N} k_{i j} A_{j}=0 \quad i=1,2, \ldots 3 N
$$

As is well known, such a set of $3 N$ simultaneous linear homogeneous equations has a solution other than the trivial one $A_{1}=A_{2}=\cdots=A_{3 N}=0$ only when the determinant of the coefficients is zero. The normal frequencies are given by the $3 N$ roots of this secular equation:

$$
\left|\begin{array}{cclc}
k_{11}-b_{11} \lambda & k_{12} & \ldots & k_{1,3 N} \\
k_{21} & k_{22}-b_{22} \lambda & \ldots & k_{2,3 N} \\
\vdots & & & \\
k_{3 N, 1} & \ldots & & k_{3 N, 3 N}-b_{3 N, 3 N} \lambda
\end{array}\right|=0
$$

i. e., the $3 N$ values of $\lambda$ which satisfy this equation. It should be noted that of these $3 N$ frequencies at least three, representing translations, are zero. If the molecule were free to rotate, there would be three more zero

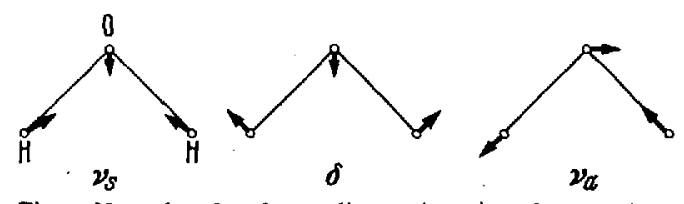

Fig. 1. Normal modes of a non-linear triatomic molecule such as $\mathrm{H}_{2} \mathrm{O}$ (schematic). $\nu_{8}$ : symmetric stretching, $\delta$ : symmetric bending, $v_{a}$ : asymmetric stretching frequencies (two in the case of a linear molecule). The form of the normal vibration for a particular mode, say $l$, is determined by substituting $\lambda_{l}$ in equation (15) and solving for the ratios of the amplitude constants $A_{k}$. These ratios are given by the ratios of the minors of any row of the determinant of equation (16) after putting $\lambda=\lambda_{l}$. An example of the resulting normal modes for a non-linear triatomic molecule, such as $\mathrm{H}_{2} \mathrm{O}$, is given in Fig. 1. The most general internal vibration of a molecule is a superposition of such normal vibrations.

The determination of the normal vibrations does not specify the conditions under which interaction with electromagnetic radiation is possible. For our purposes these may be taken to be the classical requirements. According to the latter, if there is a change in the dipole moment of the molecule during the normal vibration, there can be absorption of electromagnetic energy. The intensity of the resulting absorption band will be proportional to the square of the change in dipole moment (quantum mechanically, to the square of the transition moment). In many cases there is no change in the dipole moment of a molecule during a particular normal vibration. There will therefore be no possibility of inducing a transition in the energy state associated with this vibration by the absorption of infrared radiation. However, a transition can frequently be induced by a different mechanism, viz., Raman scattering. 
In this effect light scattered by the molecule is found to contain frequencies other than that of the incident monochromatic light; the differences between these frequencies and that of the incident light correspond to normal vibration frequencies of the molecule. Raman scattering can occur only when there is a change in the polarizability of the molecule during the normal vibration. It is important to emphasize in this connection that knowledge of the complete vibrational spectrum often requires both Raman and infrared data. This is particularly true of molecules with a center of symmetry, because the mutual exclusion rule for this case requires that vibrations which are infrared active, viz., those which are antisymmetric with respect to the center of symmetry, be Raman inactive, and vice versa [HERzBerg (76)].

The foregoing discussion has been based on the assumption of a harmonic potential function, i. e., one in which no terms higher than the quadratic ones appear. A consequence of this is that the normal modes are of the simple harmonic oscillator type, and therefore only the fundamental frequencies appear in infrared absorption and in Raman scattering. In actual molecules, of course, the forces are not strictly harmonic (if they were, infinite energy would be required to break a chemical bond). This means that cubic and quartic terms in $V$ are not negligible. When such terms are included in the potential function, the wave equation cannot be separated as before into a number of independent equations. As a consequence the total energy is no longer the sum of independent terms corresponding to the normal vibrations. A perturbation calculation shows that the total energy now contains cross terms involving the quantum numbers of two or more normal vibrations. The result of this is that overtone and combination bands can now appear in the infrared and Raman spectra. Although such bands are usually weaker than the fundamentals, they are often strong enough so that their presence cannot be neglected. It might be noted that, because the anharmonic constants are usually negative, the frequencies of combinations and overtones are generally less than the sum of the fundamentals.

The above discussion has outlined the theoretical approach to the determination of the fundamental vibration frequencies of a molecule. The practical solution of the problem as formulated above presents, however, certain more or less serious difficulties. For example, the completely general potential function of equation (4) is generally not usable even for small molecules, because it contains more independent constants than can be determined from the experimental data. However, by making certain assumptions about the nature of the force field in the molecule, the number of constants can be reduced. One assumption which often works quite well in practice is that of a valence force field [HERZBERG (76)]. This assumes that contributions to the potential energy 
arise only from the stretching of chemical bonds and from the bending of angles between chemical bonds. The advantages of such a potential function are essentially two-fold: (a) The number of independent force constants which it contains is usually smaller than the number of observed fundamental frequencies. This makes it possible to check the validity of the potential function by using some of the observed frequencies to compute the force constants and then using these constants to calculate the remaining frequencies. (b) The potential defined by a valence force field can be given a simple physical meaning. It permits the internal vibrations of a molecule to be characterized essentially as bondstretching and angle-bending vibrations. In many cases, however, such a force field turns out to be too much of an oversimplification. For example, the kind of check mentioned above may lead to calculated frequencies which deviate by as much as $10 \%$ from the observed frequencies. An improvement can be made, especially when there are fewer valence force constants than observed fundamentals, by including in the potential function interaction terms which represent the existence of weak central forces between non-bonded atoms. Such a mixed potential function was first introduced by UREY and BRADLEY (234), and has been shown to provide an adequate description of the force field in a molecule [Simanouti (199)]. It is thus possible to set up the vibrational problem in a form which can be meaningfully related to the experimental data.

The determination of the normal modes and their frequencies, however, depends upon solving the secular equation, a $3 N \times 3 N$ determinant. This rapidly becomes nontrivial as $N$ increases. Methods do exist which somewhat simplify the computational problem. Thus, if the molecule has symmetry, the $3 N \times 3 N$ determinant can be resolved into sub-determinants of lower order, each of which involves only normal frequencies of a given symmetry class. These determinants are of course easier to solve. (We will return shortly to the subject of symmetry considerations since they not only aid in the solution of the secular equation, but they permit the determination - without any other information about the molecule - of many characteristics of the normal modes, such as their number, activity in the infrared and Raman spectra, possibilities of interaction, and so on.) In addition, special techniques have been developed for facilitating the setting up and solving of the secular equation [Wilson, Decius, and Cross (245)]. Even these, however, become prohibitive for the large $N$ encountered in complex molecules such as high polymers.

\section{B. Group Frequencies}

It would seem from the previous discussion that there is little hope of interpreting the spectra of complex molecules. The solution of the 
vibrational problem is immensely difficult for systems with such a large number of particles, and it might be supposed therefore that not much could be said about the origin of the fundamental frequencies of a large molecule. The situation, in fact, is much more hopeful than appears at first sight, and this is in part a consequence of the nature of the binding forces in molecules.

Quantum-mechanical considerations of the stability of molecules [Coulson (40)] indicate that in most cases the orbitals which describe the electronic configurations within the molecule are localized and, to a first approximation, independent of one another. The localization implies that electrons are concentrated in certain characteristic directions about an atom. The strongest bonding between atoms results when there is maximum overlap between an orbital of one atom and that of another. These principles are the basis of the concept of directed valence bonds in molecules, and lead to the expectation that the force field characteristic of a particular kind of bond should remain practically invariant from molecule to molecule. Consider $\mathrm{CH}_{4}$ as an example. We know that maximum binding will occur if the valence electrons of carbon form four tetrahedrally oriented hybrid orbitals, in this case of the $\mathrm{sp}^{3}$ type. A $\mathrm{C}-\mathrm{H}$ bond results when such a localized tetrahedral orbital of carbon overlaps a 1s orbital of a hydrogen atom. If we replace one of the $\mathrm{H}$ atoms by a $\mathrm{CH}_{3}$ group, we do not to a first approximation influence the orbitals about the original carbon atom, and therefore essentially no change takes place in the strengths of the remaining $\mathrm{C}-\mathrm{H}$ bonds. This means that, in terms of a particular force field (such as a valence or a Urey-Bradley ficld), it should be possible to transfer force constants for a particular bond from one molecule to another. This has indeed been shown to be the case [SUTHERLAND and Dennison (275); Crawford and BrinkLey (42); Decius (48); Meister, Dowling, and Bielecki (130)]. It is necessary to keep in mind that this invariance of force constant is only true in first approximation, and then mainly for bond-stretching force constants. Angle-bending force constants, which are generally about an order of magnitude smaller than the stretching constants, are much more sensitive to the environment, as are interaction constants. Nevertheless, the significant point is the essential constancy of bond force constants from molecule to molecule.

The important consequence of the constancy of force constants is that, under certain conditions, the frequencies associated with the motion of particular atoms or groups of atoms will remain essentially constant from molecule to molecule. These are known as group frequencies, and they provide an important tool for the spectral characterization of a molecule. It is therefore important to inquire into the validity and limitations of this concept. 
We have already noted the necessity of similarity in environment as a prerequisite for constancy of force constants. Variations in the environment give rise to changes (usually small) in the characteristic frequency. Thus, for example, the frequencies associated with normal modes that involve primarily the stretching of the $\mathrm{C}-\mathrm{H}$ bond will depend upon whether the remaining bonds on the carbon atom are single, double, or triple, i. e., the $\mathrm{C}-\mathrm{H}$ force constant, and therefore stretching frequency, will be a function of the kind of hybridized orbitals around the carbon atom. In saturated compounds such frequencies are found in the range of roughly $2850-2950 \mathrm{~cm}^{-1}$, whereas when the carbon atom participates in a double or a triple bond this frequency occurs at approximately 3000 to $3100 \mathrm{~cm}^{-1}$ or $3300 \mathrm{~cm}^{-1}$ respectively [BeLLAMY (13)]. This represents a change in the $\mathrm{C}-\mathrm{H}$ stretching force constant of about $1 \times 10^{5} \mathrm{dynes} / \mathrm{cm}$, from about $4.9 \times 10^{5}$ dynes $/ \mathrm{cm}$ in methane to $5.9 \times 10^{5}$ dynes $/ \mathrm{cm}$ in acetylene (Coulson (40)]. Although a rather large change in the characteristic frequency occurs between these various types of $\mathrm{C}-\mathrm{H}$ bonds, it is important to note that the frequencies are all found in a fairly restricted region of the spectrum. In many cases the range is smaller than for $\mathrm{C}-\mathrm{H}$ bonds, because of the smaller variation in the electronic environment. Thus, the absorption arising from the stretching motion of the $\mathrm{C}=\mathrm{O}$ bond occurs in the region of about $1650-1750 \mathrm{~cm}^{-1}$ for a wide range of compounds containing this group. In this case there are also subranges within this range which are characteristic of the various kinds of carbonyl-containing compounds. While we understand in a general way the reasons for these small differences in the frequency associated with a given group in different molecules, we do not as yet have a detailed enough theory which would permit the a priori prediction of such frequency shifts. We may therefore say that to a first approximation the electronic environment of certain small groups of atoms remains constant from molecule to molecule, such that the force field and the vibration frequencies associated with this group are approximately invariant. In higher approximations, however, the environment is rarely identical, and as a consequence variations in the frequency associated with a group will occur. Some sources of such variability will be considered later.

While constancy of the electronic environment is necessary in order to insure transferable force constants, it is not sufficient to guarantee the existence of separable group frequencies. The essential characteristic of the latter is that in a particular normal vibration of the molecule as a whole the displacements may validly be said to be confined primarily to the atoms of this group. Not only this, but also such a group normal mode should remain basically unchanged from molecule to molecule. The existence of such a group frequency is intimately connected with the extent of interaction between the characteristic motions of this group and 
those of the rest of the molecule. When this interaction is small, it is possible to talk of a group mode; when it is large, this is no longer feasible, and we must consider the motions of all of the atoms in the molecule.

As an illustration of the factors which determine the separability of such group modes, we will consider the stretching vibrations of a linear triatomic molecule, $\mathrm{XYZ}$. Such a molecule has $3 \times \mathbf{3 - 5}=\mathbf{4}$ internal vibrational modes, of which two involve stretching of the $X-Y$ and $\mathrm{Y}-\mathrm{Z}$ bonds and the other two (which are degenerate, i. e., of the same frequency) bending of the XYZ angle. By the method outlined in section I. 1. we can obtain the frequencies and forms of the stretching modes when the masses and force constants are specified. The secular equation is

$$
\left|\begin{array}{ccc}
k_{X Y}-m_{X} \lambda & -k_{X Y} & 0 \\
-k_{X Y} & \left(k_{X Y}+k_{Y Z}\right)-m_{X} \lambda-k_{Y Z} \\
0 & -k_{Y Z} & k_{Y Z}-m_{Z} \lambda
\end{array}\right|=0
$$

the frequencies being given by

$$
\begin{gathered}
\lambda_{1,2}=4 \pi^{2} v_{1,2}^{2}=\frac{k_{X Y}}{2}\left(\frac{1}{m_{X}}+\frac{1}{m_{Y}}\right)+\frac{k_{Y Z}}{2}\left(\frac{1}{m_{Y}}+\frac{1}{m_{Z}}\right) \\
\pm \frac{1}{2} \sqrt{\left[k_{X Y}\left(\frac{1}{m_{X}}+\frac{1}{m_{Y}}\right)+k_{Y Z}\left(\frac{1}{m_{Y}}+\frac{1}{m_{Z}}\right)\right]^{2}-4 k_{X Y} k_{Y Z}\left(\frac{1}{m_{X} m_{Y}}+\frac{1}{m_{Y} m_{Z}}+\frac{1}{m_{Z} m_{X}}\right)}
\end{gathered}
$$

The results, for several values of the masses and force constants, are given in Table 1, where we have chosen the masses to correspond roughly to $\mathrm{H}, \mathrm{C}$, and $\mathrm{N}$ atoms and the force constants to be approximately those of single, double, and triple bonds. In the drawings of the normal modes the scale of the displacements is not the same as that of the internuclear

\begin{tabular}{|c|c|c|c|c|c|c|c|c|c|c|}
\hline & $\dot{m}_{x}$ & $m_{Y}$ & $m_{z}$ & $k_{X Y}^{* *}$ & $k_{Y Z}$ & & Mode & $\begin{array}{c}\text { Frequency } \\
\mathrm{cm}^{-1}\end{array} \mid$ & $V_{X Y}$ & $V_{X Z}$ \\
\hline I. & 1 & 12 & 12 & 5 & 5 & $\begin{array}{l}\nu_{1}: \\
\nu_{2}:\end{array}$ & $\underset{1.6}{\stackrel{2.5}{a .3}} \stackrel{2.5}{\longrightarrow .5}$ & $\begin{array}{l}3030 \\
1170\end{array}$ & $\begin{array}{r}98.7 \\
0.7\end{array}$ & $\begin{array}{r}1.3 \\
99.3\end{array}$ \\
\hline II. & 12 & 12 & 14 & 5 & 5 & $\begin{array}{l}v_{1}: \\
\nu_{2}: \frac{\overleftrightarrow{35}}{27.2}\end{array}$ & $\underset{2.1}{\frac{\overrightarrow{20}}{25.0}}$ & $\begin{array}{r}1435 \\
810\end{array}$ & $\begin{array}{l}55.7 \\
48.0\end{array}$ & $\begin{array}{l}44.3 \\
52.0\end{array}$ \\
\hline III. & 12 & 12 & 14 & 5 & 10 & 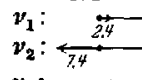 & $\frac{8.2}{1.5}$ & $\begin{array}{r}1790 \\
927\end{array}$ & $\begin{array}{l}24.3 \\
75.8\end{array}$ & $\begin{array}{l}75.7 \\
24.2\end{array}$ \\
\hline IV. & 12 & 12 & 14 & 10 & 5 & 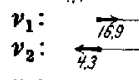 & $\begin{array}{r}\frac{12.6}{1.7} \\
-\frac{1}{2}\end{array}$ & $\begin{array}{r}1830 \\
903\end{array}$ & 80.5 & $\begin{array}{l}19.5 \\
76.5\end{array}$ \\
\hline v. & 12 & 12 & 14 & 5 & 15 & $\begin{array}{l}v_{1}: \\
v_{2}: \frac{\pi}{2.6}\end{array}$ & 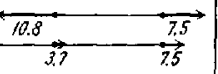 & $\begin{array}{r}2100 \\
960\end{array}$ & $\begin{array}{l}14.4 \\
86.0\end{array}$ & $\begin{array}{l}85.6 \\
14.0\end{array}$ \\
\hline VI. & 12 & 12 & 14 & 15 & 5 & $\begin{array}{l}\nu_{1}: \\
\nu_{2}: \overleftrightarrow{4.20}\end{array}$ & $\underset{7.5}{\stackrel{7.5}{\longrightarrow}}$ & $\begin{array}{r}2170 \\
930\end{array}$ & $\begin{array}{l}88.5 \\
11.7\end{array}$ & $\begin{array}{l}11.5 \\
88.3\end{array}$ \\
\hline
\end{tabular}
distances, nor is the scale of displacements the same in all modes. Only

Table 1. Stretching Modes of a Linear XYZ Molecule 
in a given mode are the relative displacements in the proper ratio. The last two columns of the table give the percentage of the energy of the vibration which is localized in the stretching of the $\mathrm{X}-\mathrm{Y}$ and $\mathrm{Y}-\mathrm{Z}$ bonds respectively.

This example illustrates several characteristic features of separable group frequencies. First, as can be seen from case $I$ in the table, when one of the masses is small in comparison with the others, one mode of vibration of the system involves essentially the motion of only this mass. This is true even when the force constant for the motion of this mass is comparable to those in other parts of the molecule. Another way of seeing this is to observe that when, say, $m_{X}<m_{Y}$, $m_{Z}$, equation (18) gives

$$
\lambda_{\mathrm{I}}=4 \pi^{2} v_{1}^{2} \cong \frac{k_{X Y}}{m_{X}} .
$$

That is, one vibration corresponds essentially to the motion of the light atom against an infinite mass, and therefore to a first approximation it will be independent of the nature of this mass. This is why the stretching vibration of a $\mathrm{H}$ atom can generally be treated very satisfactorily as a separable group frequency. Second, when the masses and force constants are roughly the same throughout a molecule, one usually cannot talk of group frequencies. As can be seen from the $V$ values in case II of Table 1, both modes contain substantial contributions from the stretching of both the $\mathrm{X}-\mathrm{Y}$ and $\mathrm{Y}-\mathrm{Z}$ bonds. In general, in such a case all of the atoms will have significant displacements during the normal vibration, and one cannot consider the motion to be localized in one group of the molecule. The determining factor with respect to localization is the degree of coupling between the groups. In the above case this coupling is strong, because the two groups share a common atom. The result is a resonance between the two vibrations, with resultant modes which represent essentially in-phase and out-of-phase combinations of the vibrations of the individual groups. The frequencies of these modes generally fall both above and below the frequency of the single group. This is evident in case II, where the interacting oscillators ( $\mathrm{X}-\mathrm{Y}$ and $\mathrm{Y}-\mathrm{Z}$ ) are practically of the same frequency (about $1190 \mathrm{~cm}^{-1}$ and $1150 \mathrm{~cm}^{-1}$ for $X-Y$ and $\mathrm{Y}-\mathrm{Z}$ respectively, taken as diatomic molecules). This will, of course, also be true of two identical groups in the same molecule whose vibrations can couple. Thus, in the case of the $\mathrm{H}$ stretching modes in $\mathrm{H}_{2} \mathrm{O}$ (see Fig. 1) we cannot talk of separate stretching vibrations for each $\mathrm{O}-\mathrm{H}$ bond, but expect to find two vibrational modes in each of which both $\mathrm{H}$ atoms move. While this coupling is naturally expected when groups are adjacent to each other, we will see that it can also occur when the groups are separated by several atoms and even when they are on different molecules. On the other hand, in many cases proximity does not necessarily lead to 
strong coupling. Thus, the presence of a benzene ring in a molecule usually results in the retention of identity of most of the characteristic modes of the ring [RANDLE and WhIFFEN (183)]. Each case must be considered individually. The only general rule which must hold in all cases is that the vibrations which couple must be of the same symmetry [HERZBERG (76)]. Third, when the masses in a molecule are of the same order of magnitude but the force constants differ significantly, separability of group frequencies is again approached. This is seen by comparing cases II and IV in Table 1, where the change in one force constant from a single to a double bond value has changed the potential energy distribution in the stretching of the two bonds in $v_{1}$ from about $55 \%-45 \%$ to $80 \%-20 \%$. It is important to note, incidentally, the sense in which we speak of group frequencies: we require that a major portion of the energy of the vibration be localized in the motion of a small group of atoms, but often this is appreciably less than $100 \%$. Significant displacements of atoms other than those in the group do occur. In some cases, such as determining the direction of the transition moment, it is important to know such details of the normal vibration; unfortunately our present knowledge of this aspect of normal modes in complex molecules is quite meager.

The above example illustrates the general principle that the group frequency concept is most valid when there is minimum interaction between the characteristic vibrations of the given group and those of the rest of the molecule. That this situation exists in first approximation in complex molecules is attested to by the fact that numerous correlations have been worked out from the detailed study of smaller molecules and have been successfully applied to the study of larger molecules [RANDALL, Fowler, Fuson, and Dangl (782); Colthup (37); Bellamy (13); Jones and SANDORFY, (82)]. Most spectroscopic studies of high polymers have been characterized mainly by an effort to identify such well-known group frequencies in the infrared spectrum [HummeI (81)]. However, when we examine the spectrum in greater detail, particularly from the point of view of attempting to achieve a complete assignment, it becomes clear that group modes in complex molecules are often significantly modified in form, frequency, and intensity. In order to achieve a detailed understanding of the spectra of large molecules we must therefore give consideration to the specific kinds of factors which affect such modes. There have been several discussions of some of these factors [LORD and Miller (120); Bellamy (13)]. We will review briefly those which are most pertinent in polymer spectra.

Mass Effects. Changes in the mass distribution in a portion of a molecule, either in magnitude or in the configuration, can alter the form as well as the frequency of the normal vibration. For example, in cases III and 
IV of Table 1 a small change in the masses (with respect to the potential field) has altered the potential energy distribution from about $75 \%-25 \%$ to $80 \%-20 \%$. The effect in this instance is quite small because of the small change in mass, but for a larger mass change (such as the substitution of $\mathrm{D}$ for $\mathrm{H}$ ) it could be quite significant. In such a case not only will there be a more pronounced change in the vibration from one characteristic of one group to one characteristic of another, but bondstretching modes can change to bond-bending modes [HERzBERG (76), p. 200; Kohlrausch (92), p. 227]. (This cannot happen in the present case because the two types belong to different symmetry species, and no mixing of modes of different symmetry can occur.)

Although this effect is generally not a significant factor when dealing with the modes of most small groups within a large molecule [cf. the case of the carbonyl group, HALForD (71)], there are cases where it is quite important. For example, although the skeletal modes of planar zig-zag polymer chains are usually treated as if they were similar in all instances it is clear that this cannot be the case. Thus, the modes of a polyvinyl chloride chain will not be the same as those of a polyethylene chain: the substitution of a $\mathrm{Cl}$ atom for a $\mathrm{H}$ atom would be expected to alter somewhat the form as well as the frequency of the modes of the carbon backbone. We presume that this is not an effect of major magnitude, but unfortunately little work has been done to study the quantitative effects of mass variations in such a case (to say nothing of the skeletal modes of non-planar-zig-zag chains). We must recognize the possible existence of such an effect, but as yet we can only treat it qualitatively.

A situation in which the mass configuration plays a dominant role is rotational isomerism. Rotation around a $\mathrm{C}-\mathrm{C}$ single bond is in general not completely free. For example, in ethane there is a barrier of height about $3 \mathrm{kcal} / \mathrm{mole}$ hindering rotation [HERzBERG (76)]. A similar barrier exists when all of the substituents are not the same, as for example in 1,2 dichloroethane[Mizushima (137); SHEPPARD (194 a)]. Such hindered rotation about. a C-C bond is characterized by a potential with three minima, corresponding in the case of 1,2 dichloroethane to one configuration in which the two $\mathrm{Cl}$ atoms are trans to each other and two configurations in which they are gauche [Mrzushrma (137)]. A barrier of the above height is not large enough to stabilize such rotational isomers: interconversion between the isomeric forms is very rapid in the gas or liquid phase at ordinary temperatures, even though one of the isomers may be of lower energy than the others. The molecule does, however, spend enough time in each of its isomeric states so that many oscillations of any given normal vibration can occur. The possibility therefore exists that rotational isomers will be spectroscopically distinguishable. This is in fact often the case, and is due primarily to the different normal frequencies which are to be expected for 
the different mass configurations, the latter being essentially different structures. Thus, for example, the $\mathrm{C}-\mathrm{Cl}$ stretching frequencies of the trans form of 1,2 dichloroethane are predicted at $797 \mathrm{~cm}^{-1}$ and $737 \mathrm{~cm}^{-1}$ while the corresponding ones of the gauche form are expected at $671 \mathrm{~cm}^{-1}$ and $691 \mathrm{~cm}^{-1}$ [Mizushima (137)].

There are two aspects of rotational isomerism that are worth emphasizing. First, crystallization will usually result in the stabilization of the lowest energy isomer, whereas all forms are present in the liquid. Since the amorphous state in polymers is essentially a disordered state comparable to liquids, the spectrum of a partially crystalline polymer in which rotational isomerism can occur will be a superposition of the spectra of the various isomers. Second, rotational isomers generally have different symmetry, so that the number of modes associated with a given group can be different for the various isomers. Thus, the trans form of 1,2 dichlorethane has a center of symmetry while the gauche form does not, and therefore, in accordance with the mutual exclusion rule, the former will exhibit only one infrared active $\mathrm{C}-\mathrm{Cl}$ stretching mode while the latter will have two. (It might be noted in this connection that any stabilized departure of a molecular configuration from the one having a center of symmetry would be expected to give rise to new infrared bands.) Both of these effects seem to occur in the spectra of high polymers.

Electrical Effects. Any agent which affects the electron distribution in a bond will obviously influence the force constant and therefore the characteristic vibrational frequency of the atoms associated with this bond. Such electrical effects can arise from internal or external influences on the molecule. The effect of neighboring electronegative groups in the molecule is an example of the former; the dielectric effect of a solvent, the electric field of a crystal, and hydrogen-bonding are examples of the latter. With respect to internal effects, atoms are known to differ in their electron-attracting power, or electronegativity [Coulson (40)]. It is therefore to be expected that the characteristic frequencies, and possibly also intensities, of a group will be modified when it is adjacent to atoms of varying electronegativity. In some cases, as for example with the carbonyl group [KAGARISE (83)], it has been possible to show that the frequency shift is quantitatively related to the electronegativities of the adjacent substituents. This is also true of $\mathrm{CH}$ frequencies when this group is adjacent to, for example, halogen atoms [WILMSHURST (244)]. In other cases the arguments are less quantitative. The situation is thus one in which the occurrence of the effect can frequently be predicted in complex molecules but the magnitude in general cannot. With respect to external electrical effects, the influence of solvents in lowering the vapor phase frequency is very small, and will only be of interest when polymer 
solution spectra are under consideration. Other external electrical effects will be considered later.

Intramolecular Interactions. As was pointed out above, whenever two group vibrations in a molecule have frequencies near each other, the possibility exists (unless they belong to different symmetry species) that interaction between them will occur. The effect of this interaction is to mix the two modes, giving normal vibrations which are no longer describable in terms of a single group alone. This can occur when the two groups are identical and situated near each other (e. g., two $\mathrm{CH}_{3}$ groups attached to the same carbon atom) so that their motions can couple. Such interaction can also take place between similar or dissimilar modes of different groups, such as between stretching vibrations of heavy groups and bending vibrations of lighter units. We have little detailed information on such cases, although some is beginning to appear. For instance, the assignment of the in-plane deformation frequency of the $\mathrm{H}$ atom in the $\mathrm{OH}$ group has been the subject of much inquiry [SUTHERLAND (213); TARTe and DeponthiEre (224)]. Recent studies on the spectrum of polyvinyl alcohol have indicated [KRIMM, LIANG, and SUTHERLAND (103)] that the $\mathrm{OH}$ deformation mode of the $-\mathrm{C}_{\mathrm{OH}}$ group of primary and secondary hydroxyl compounds probably interacts and mixes with the $\mathrm{C}-\mathrm{H}$ deformation mode associated with the common carbon atom. This has been confirmed by a detailed study of the normal vibrations of methanol [Tanaka, Kuratani, and Mizushima (223)]. We therefore know that the $\mathrm{OH}$ deformation mode is not an isolatable group frequency in such molecules. A similar problem has existed with respect to the frequencies associated with the amide group, - CONH- [SUTHERLAND (214); Bellamy (13)]. In this case the NH deformation and CN stretching modes are expected to have frequencies near each other, and it was pointed out [FRASER and PRICE (62)] that the various modes of the amide group are therefore probably strongly coupled. This has been substantiated by a recent detailed analysis of the normal vibrations of N-methylacetamide [Miyazawa, Shimanouchi, and Mizushima (136)], in which the forms of the normal modes have been obtained. The analysis clearly shows that the supposition of separable CO stretching, $\mathrm{CN}$ stretching, and $\mathrm{NH}$ deformation modes for the amide groups is an oversimplification, and that the observed frequencies actually derive from normal modes which represent combinations of these various motions. In a sense, separable frequencies can be associated only with the - CONH - unit, not with its component groups. We see, therefore, that caution is often necessary in attempting detailed assignments in complex molecules: the form of the normal vibration may be significantly altered 
as a result of intramolecular interactions, with consequent changes in the frequency associated with the group of atoms in question.

Intermolecular Interactions. The electrical forces associated with intermolecular interactions are usually (except in cases like hydrogen bonding, which we will consider separately) much weaker than intramolecular forces. We might therefore expect that normal modes and their frequencies would be altered relatively little when molecules in the gas phase are condensed to the crystalline state. This is indeed found to be the case, in the absence of specific effects like hydrogen bonding [HoRNIG $(79,80)$; HALFORD $(73)]$. The implication of this result with respect to polymers is that in general the normal frequencies of chains in the amorphous phase will be nearly the same as those in the crystalline phase of the specimen. (The infrared and Raman activity of some modes of amorphous chains may of course be different from their activity in the crystalline chain as a result of changes in the symmetry of the molecule. Since the symmetry is generally lower in the amorphous state, we expect, as we shall see later, more bands to be present in the amorphous state spectrum.) Although frequencies are not significantly affected by crystallization, band contours and intensities may be altered, and dichroic properties of the ensemble of molecules may no longer be simply related to those of local groups. We will discuss the latter effect in more detail later. A few remarks on the former are in order here.

The effect of intermolecular interactions on molecules in the crystalline state (where such interactions are most specific) is best approached by a consideration of the vibrational spectra of crystals. The solution to this problem proceeds in a manner similar to that for a molecule. The potential energy, which is of salient interest here, is given by [HORNIG $(80)]$

$$
V=V_{L}+\sum_{j}\left(V_{j}^{0}+V_{j}^{\prime}\right)+\sum_{j} \sum_{k} V_{j k}^{\prime \prime}+V_{L j}^{\prime \prime \prime}
$$

where $V_{L}$ is the potential function of the lattice, i. e., the potential energy when the molecules are considered as rigid bodies; $V_{j}^{0}$ is the potential energy function of a free molecule, and corresponds to equation (3); and $V_{j}^{\prime}, V_{j k}^{\prime \prime}$, and $V_{L_{j}^{\prime \prime}}^{\prime \prime}$ are perturbations on $V_{j}^{0}$. The first of these, $V_{j}^{\prime}$, describes the effect of the equilibrium field of the crystal on $V_{j}^{0}$; the second represents the interaction between the internal displacement coordinates of the $j^{\text {th }}$ and $k^{\text {th }}$ molecules; and the third represents the interaction between internal and lattice motions. The interaction terms are usually small. If they are neglected entirely, the problem can be resolved, because of the usually large difference in frequency, into problems of the lattice vibrations and the internal modes of free molecules. We have already considered the latter. The former has also been worked out in detail [BorN and HuANG (18)], though not in the case of polymers (such lattice 
vibrations are responsible for the long wavelength reststrahlen frequencies of ionic crystals). With the inclusion of the $V_{j}^{\prime}$ terms the problem is still separable [HoRNIG (79)], but deviations from the spectrum of a free molecule are now possible. In the first place, the change in the magnitude of the potential field at the molecule can lead to a frequency shift for some of the internal modes of the molecule. Since the crystal field is generally weak in comparison with the internal field of the molecule, such frequency shifts are usually small. Second, because the symmetry of the potential field in which the molecule finds itself in the crystal can be lower than that of the free molecule, changes in selection rules can occur which will permit for example the appearance of new bands or the splitting of degeneracies. With respect to polymers, this is most likely to occur in comparing the spectrum of the crystal with that of an isolated chain having the same configuration as in the crystal [LIANG, KRIMm, and Sutherland (117); Krimm, Liang, and Sutherland (102)]. In comparing spectra of the amorphous and crystalline polymers, since there is usually an increase in the symmetry of the molecule on crystallization there will often be fewer bands in the latter case. Third, in the anharmonic oscillator approximation, combinations of internal and lattice modes are permitted [HoRnig (79)]. The results of this can be that bands are broadened in the crystal, and that forbidden fundamentals may seem to be present because of the occurrence of their allowed combinations with lattice modes. Such effects have not been unambiguously identified in the spectra of polymers.

If the crystal contains $\mathrm{N}$ molecules per unit cell, the solution of the vibrational problem in the above approximation will yield $\mathrm{N}$-fold degenerate crystal modes for each non-degenerate normal vibration of free molecule. The addition of the $V_{j k}^{\prime \prime}$ term in the potential energy can resolve this degeneracy, In other words, a given internal mode in a molecule gives rise to $\mathrm{N}$ crystal modes in which this internal vibration is repeated in the $\mathrm{N}$ molecules of the unit cell with different relative phases consistent with the symmetry of the crystal. When $V_{j k}^{\prime \prime}=0$ the frequencies of all of these crystal modes are the same. When $V_{j k}^{\prime \prime} \neq 0$ the possibility exists that the frequencies of these modes will be different, the magnitude of the splitting being a function of the strength of this coupling. A splitting of this kind seems to occur in the spectrum of n-paraffins and polyethylene [KRIMM (93)]. The effect of the lattice vibrations on the internal modes, given by $V_{L j}^{\prime \prime \prime}$, seems to be negligible [HORNIG (80)], and we will therefore leave it out of consideration.

In summary, we see that intermolecular interactions such as occur in the crystalline state are not likely to affect greatly the frequencies of group normal vibrations, but they will influence the detailed structure of the absorption band associated with a given mode as well as its intensity. 
Hydrogen Bonding. A special case of an intermolecular interaction (which in some instances can be intramolecular) with a large influence on a group frequency is hydrogen bonding. This interaction, with energies of the order of $5 \mathrm{kcal} / \mathrm{mole}$, is much weaker than the energies of covalent bonds, of order $100 \mathrm{kcal} /$ mole [STUART (211)], but it is large enough to give rise to appreciable shifts in frequency (of the order of $100 \mathrm{~cm}^{-1}$ ). Hydrogen bonding involves the specific interaction between an $-\mathrm{X}-\mathrm{H}$ bond on one molecule and a $\mathrm{Y}$ atom on another. It is favored by highly electronegative $\mathrm{X}$ atoms $(\mathrm{O}, \mathrm{N}, \mathrm{F})$, and requires $\mathrm{Y}$ atoms with lone pair electrons [Cannon (37); Pimentel and McClellan (172a)]. The interaction, which may be described schematically by $-\mathrm{X}-\mathrm{H} \cdots \mathrm{Y}-\mathrm{Z}-$, is partially electrostatic and partially quantum-mechanical in origin. Its primary effect is to alter the stretching and deformation frequencies of the $-\mathrm{X}-\mathrm{H}$ and $\mathrm{Y}-\mathrm{Z}-$ groups, although changes in band intensity and width also occur. As a result of the force of attraction represented by the weak $-\mathrm{H} \cdots \mathrm{Y}-$ bond, the stretching frequencies of the $-\mathrm{X}-\mathrm{H}$ and $\mathrm{Y}-\mathrm{Z}-$ groups will be lowered on hydrogen bonding, whereas the deformation frequencies (associated with the motion of the $\mathrm{H}$ and $\mathrm{Y}$ atoms perpendicular to their respective bonds) will be increased. The magnitude of the frequency shift depends on the strength of the $-\mathrm{H} \cdots \mathrm{Y}$ - bond, and can therefore be related to the $\mathrm{X}-\mathrm{Y}$ distance [LIPPINCOTT and Schroeder (119)]. In some cases, such as the $-\mathrm{N}-\mathrm{H} \cdots \mathrm{O}=\mathrm{C}-$ bond, a colinear arrangement of all four atoms is expected to be most stable [PAULING (164)] and it appears that deviations from such colinearity give rise to interpretable variations in the frequency shift [KrImM (95)]. Hydrogen bonding effects are usually easy to identify in the spectrum because we can predict their presence on the basis of the chemical structure of the molecule.

In summarizing this discussion of group frequencies, we may say that experimentally and theoretically it is expected that the vibrational frequencies of particular groups of atoms will remain relatively constant from molecule to molecule. It is this principle, for example, which validates the procedure of identifying group modes in large molecules by comparing their frequencies with smaller molecules in which the vibrations of this group have been determined in detail. On the other hand, many factors operate to modify group frequencies and normal modes, more or less strongly depending upon the nature of the environmental influences. These become particularly important when we utilize the concept of group frequencies as a tool in undertaking a complete assignment of a spectrum. We turn to this more general approach to the interpretation of spectra after a brief consideration of experimental techniques. 


\section{Some Experimental Techniques}

The general techniques used to obtain infrared spectra have been covered in detail elsewhere [BRÜGEL (24); KAYE (85); CLARK (35); Smith, Jones, and Chasmer (203); LecomTe (109)], and we will therefore not discuss them here. Some remarks are, however, in order with respect to particular methods pertinent to the investigation of high polymer spectra, as well as areas in which further development in experimental techniques is clearly needed [see also EILIOTt (57a)].

\section{A. Far Infrared Spectroscopy}

The operation of several commercial spectrometers is described in the above references. In general, these instruments had been designed for use in the spectral region covered by a $\mathrm{NaCl}$ prism, viz., at frequencies above about $650 \mathrm{~cm}^{-1}$, although operation with a $\mathrm{KBr}$ prism down to $400 \mathrm{~cm}^{-1}$ was possible. As a result, the infrared spectra of high polymers have rarely been examined at frequencies below $650 \mathrm{~cm}^{-1}$. The first systematic investigation of polymer spectra in the low frequency region was begun in 1950 [KRIMM and SUTHERLAND (100)], using a grating spectrometer [RANDALL (181)]. Since spectra in this region are important for the purpose of complete vibrational assignments, we will review briefly the experimental techniques presently available for study in this region.

The difficulties associated with obtaining low frequency infrared spectra arise from several factors. First, suitable dispersive materials for use in prisms do not exist over the desired range (say, down to $50 \mathrm{~cm}^{-1}$ ). Recent development of cesium iodide prisms, which can be used in commercial spectrometers, has extended the workable region down to about $185 \mathrm{~cm}^{-1}$ [ACQUiSTA and PLYLER (2)], but gratings must be used for the lower frequencies. Nevertheless, the use of a cesium iodide prism provides a significant extension of the observable spectrum, one which has not yet been fully taken advantage of. Second, because of the blackbody emission characteristics of incandescent solids, the usual sources give relatively little energy at the longer wavelengths. This gives rise to two problems. Not only is the detection problem a more difficult one because of the small amount of energy available, but a more serious problem, with grating instruments, is the large amount of energy present in the unwanted higher spectral orders. For example, it has been estimated [Oetjen, Haynie, Ward, Hansler, Schauwecker, and Bell (162)] that at $100 \mathrm{~cm}^{-1}$ the ratio of unwanted to wanted radiation passing through the exit slit is of the order of magnitude of $10^{5}$. The elimination of such unwanted higher orders is one of the main problems of far infrared spectrometry. It is accomplished by judicious use of gratings, filters, beam choppers, and reststrahlen reflection plates [MCCuBbin and Sinton 
(128); Oetjen, Haynie, Ward, Hansler, Schauwecker, and Bell (162); Liang, KrImM, and Sutherland (117); LoRd and McCubbin (121)]. In this way far infrared radiation of acceptable purity can be obtained, if somewhat at the expense of energy. The energy factor is much less critical in studying polymer spectra, since absorption bands are inherently much broader than for small molecules in the vapor phase, and therefore it is possible to use wider slits. Third, detectors such as ordinary thermocouples become less efficient at the longer wavelengths because they do not absorb the radiation as efficiently as at the shorter wavelengths, i. e., they are not as "black". It becomes necessary to use a Golay cell for most efficient detection at long wavelengths. Fourth, water vapor has a very intense rotational spectrum in this low frequency region. It is therefore necessary to dry the air in, or evacuate, the spectrometer in order to observe the spectrum of a sample.

Despite these various difficulties attending spectral study in this region, several spectrometers have been built and operated successfully. Some of these have been large grating instruments [RANDALL (181); McCubbin and Sinton (128); Oetjen, Haynie, Ward, Hansler, Schauwecker, and Bell (162); YoshinaGA et al. (252)] whereas others have used small gratings in combination with commercial instruments [Lord and McCubbin (121); Plyler and Acouista (175); Plyler and BlaINe (176)]. A commercial far infrared spectrometer appears to be under development [Marshall and MikLus (124)]. The importance of extending spectral observations into the low frequency region cannot be overemphasized, particularly in connection with making complete frequency assignments. The Raman spectroscopy of polymers, which would furnish with relative ease information on some of the vibrational modes in this region, is beset with problems related to the high fluorescent background which presumably results from impurities [NIELSEN and WoolletT, (160); Simon, MÜCKIICH, Kunath, and Heintz (201)]. In any event, Raman spectra often provide only part of the necessary data. It is to be hoped that the near future will witness more extensive studies of polymer spectra in the far infrared.

\section{B. Polarization Measurements}

The study of oriented polymers with polarized infrared radiation is an equally important tool in the detailed analysis of the vibrational spectrum, since it permits us, within certain restrictions, to determine the orientation (with respect to the molecular structure) of the transition moment for a given normal mode. This makes it possible, as we shall see, not only to classify bands in the spectrum but to establish their origin. Although polarizers are available with commercial spectrometers, their use has not yet become as general as would be desirable. Some comments 
on the techniques and problems associated with such measurements may therefore be useful.

Although under certain conditions it may be feasible to study orientation effects with unpolarized radiation [STEWART (210)], such studies on polymers are most effectively achieved with polarized radiation. Polarized infrared radiation is obtained by reflection of a beam from a suitable surface set at Brewster's angle. The reflected or transmitted beam (if the surface is transparent to the radiation) can be used. Although the reflected beam is of higher purity, recent designs have favored the transmission polarizer because of the simpler optical geometry involved. If a number of sheets (say six) of the material is used, the transmitted beam can be made fairly pure without an inadmissible loss of intensity. Until recently most thought has been given to polarizers for the $\mathrm{NaCl}$ region of the spectrum. These have used either thin films of selenium deposited on a supporting membrane [PFund (170); Ellrott, Ambrose, and Temple (52); Conn and Eaton (38)] or thin sheets of silver chloride [WRIGHT (250); Newman and Halford (756); MAKas and ShurclifF (123)]. These materials are not transparent in the far infrared, and the gradual extension of observations has motivated a search for polarizers suitable for this region. One method is to use materials such as KRS-5 [LAGEMANN and Mrller (108)] and polyethylene [Shimanouchi and KAWANO (195a)] which are transparent at the longer wavelengths. Another avenue which has been explored is the use of gratings to polarize the radiation. For example, radiation reflected from an echelette grating at angles near or greater than the blaze angle contains a higher proportion of the component with electric vector perpendicular to the grooves [Peters, ZipF, and Derbel (769)], and this has been used to obtain qualitative polarization measurements at about $200 \mathrm{~cm}^{-1}$ [LIANG and KRIMM (111)]. Wire strip gratings ruled on polyethylene film have apparently been successfully used as polarizers in the 100 to $20 \mathrm{~cm}^{-1}$ region [MERTZ (732)]. Essentially no polarization studies have been made on polymers at frequencies lower than $200 \mathrm{~cm}^{-1}$, and relatively few measurements are available in the 650 to $300 \mathrm{~cm}^{-1}$ region, the region recently opened up by new prism materials. This represents a significant gap in the data, since it is particularly in the low frequency region where group frequency assignments have been least studied and are least secure.

The above remarks have been concerned with the experimental aspects of qualitative polarization measurements. Such measurements permit the determination, for example, of whether the transition moment associated with a given band is parallel or perpendicular to the orientation direction of the polymer. Relatively little consideration has been given, however, to quantitative polarization measurements, which are 
often of importance in providing structural information and in verifying assignments. The experimental quantity which is related to the orientation of the transition moment for a given mode is the dichroic ratio of the corresponding absorption band. The dichroic ratio is defined by

$$
R_{0}=\frac{\int_{0}^{\infty} \varepsilon_{\pi}(v) d v}{\int_{0}^{\infty} \varepsilon_{\sigma}(v) d v}
$$

where $\varepsilon_{\pi}(v)$ and $\varepsilon_{\sigma}(v)$ are extinction coefficients for plane polarized radiation incident normal to the orientation direction and with the electric vector oriented respectively parallel and perpendicular to this direction. It can be shown [Fraser (58)] that, if the absorption band may be represented by a Lorentz type equation, viz.,

$$
\varepsilon(v)=\frac{a^{2}}{\left(\nu-v_{0}\right)^{2}+b^{2}}
$$

where $v_{0}$ is the frequency at maximum absorption and $a$ and $b$ are constants, then

$$
R_{0}=\frac{\varepsilon_{\pi}\left(v_{0}\right)}{\varepsilon_{\sigma}\left(v_{0}\right)}
$$

The experimentally determined dichroic ratio is

$$
R=\frac{D_{\pi}\left(\nu_{0}\right)}{D_{\sigma}\left(\nu_{0}\right)}
$$

where $D$ is the optical density, $\log _{10}\left(I_{0} / I\right), I_{0}$ being the incident intensity and $I$ the transmitted intensity. The problem in obtaining quantitative values of the dichroic ratio is that $R$ can differ from $R_{0}$ for various reasons, related to the characteristics of the spectrum, the sample, and the spectrometer.

If the absorption band in question is overlapped by neighboring bands, it is clear that the determination of the true values of $D_{\pi}\left(v_{0}\right)$ and $D_{\sigma}\left(v_{0}\right)$ can be ambiguous. In such cases it is necessary to resolve the band of interest from the complex absorption of which it is a part. This is usually done by a graphical analysis, which assumes symmetrical bands of a Lorentz shape centered at the absorption maxima and simple summation of these to give the observed spectrum. The results of such a resolution are of course subject to the uncertainty of the true band shape and width, so that an indeterminate, if nevertheless small, error can be introduced in this manner.

With respect to the sample, several problems arise. First, reflection at the surface and scattering in the interior will modify the $I_{0}$ value from what it is observed to be in the absence of a sample. These losses are usually corrected for by subtracting from the peak optical density the optical density in a nearby region which is free of absorption bands (if 
such can be found), but because of the rapid change of refractive index with frequency through the absorption band this procedure is subject to unknown uncertainties. The reflection problem is apparently not too serious for unoriented polymers: a determination [RoBINSON (186)] of the extinction ccefficient for the $2850 \mathrm{~cm}^{-1}$ band of polyethylene gives a value of 0.24 from reflection measurements and 0.25 from transmission measurements. It may, however, be important for oriented specimens: if the slight increase noted above in the transmission measurement varies with the direction of polarization of the incident radiation, then the measured dichroic ratio will be in error. No careful study has been made of the latter effect. Second, for birefringent specimens (a property characteristic of oriented polymers in the infrared as well as in the visible) the propagation of a beam of plane polarized radiation can be different in the interior of the specimen from what it is in air. In particular, if the electric vector of the incident light is not parallel to a principal axis of the (complex) refractive index ellipsoid, then the incident beam of plane polarized radiation will be resolved into two plane polarized components at right angles to each other whose orientation is determined by the symmetry of the crystal structure. Thus it would be possible for absorption to occur in a crystal even if, for example, the electric vector in the incident radiation were perpendicular to a transition moment in the sample. This difficulty has been noted before [NEWMAN and HALFORD (157)], as well as the observation that when the incident electric vector is parallel to a principal axis the radiation will traverse the specimen with its plane of polarization unaltered. This means that in the case of oriented polymers, which usually exhibit uniaxial or double orientation of their crystallites (the latter usually being small compared to the wavelength), optical density measurements made with the electric vector parallel or perpendicular to the fiber axis will be meaningful, but the same will not be true if the electric vector makes any other angle with the fiber axis. The symmetry of such oriented polymer specimens determines that, although maximum absorption occurs when the electric vector is parallel to a transition moment, for the gross specimen maximum absorption occurs with the electric vector along or perpendicular to the fiber axis. It might be noted that for bands with low extinction efficient (in a single crystal) the direction of maximum absorption always coincides with a principal direction regardless of the orientation of the transition moment [WARD, (239)]. Third, the specimen may exhibit form dichroism. An isotropic absorbing material of anisotropic shape will exhibit dichroism when embedded in a medium of different refractive index [WIENER (241)]. Obviously this must be subtracted from the observed dichroism in order to obtain the structural dichroism. The theory, however, has been worked out only for dilute systems in which definite phase boundaries exist, so 
it is difficult to evaluate the importance of this factor for the case of samples such as polymer films.

The spectrometer contributes at least three kinds of possible errors to the measurement of the true dichroic ratio. Of these, imperfect polarization by the polarizer and polarization by the spectrometer are probably the most important [CHARNeY (33)]. The radiation from a transmission polarizer will always contain a small percentage of the unwanted component, about $\mathbf{7 \%}$ in the case of a six-plate $\mathrm{AgCl}$ polarizer. Because the incident radiation strikes the prism at angles other than $90^{\circ}$, the prism acts as a polarizer. The operation of these two factors is such as to reduce the magnitude of the dichroism, the measured dichroic ratio deviating by as much as a factor of two from the true value [CHARNEY (33)]. This is true even if the second factor is eliminated by setting the two planes of polarization at $45^{\circ}$ to the spectrometer slit [ElLIOTT, AMBrose, and Temple (52)]. Only if one has a perfect polarizer is the dichroic ratio independent of the polarization introduced by the prism. It might be noted that the situation is more complex in the case of a grating than for a prism, because in the former instance the intensity ratio of the two polarized components transmitted by the spectrometer is a function of the angle of incidence on the grating and can be greater or less than one [Yamaguchi, Ichishima, and Mizushima (251)]. The third instrumental factor is the convergence of the beam, which has been investigated in detail [WoOD and MITRA (249)]. The effect on the dichroic ratio is small for oriented samples as usually examined, but can be quite large in the case of a transition moment oriented parallel to the incident beam direction.

If computations of orientation and transition moment direction are to be meaningfully correlated with the observations, it is clear that factors of the type just discussed must be carefully taken into consideration.

\section{Specimen Preparation}

For purposes of a complete analysis of the infrared spectrum of a polymer, the ideal specimen would be one in which all of the chains are perfectly ordered along their length and in the same relative orientation to the beam. Although something approaching this can be achieved with low molecular weight crystallizable analogs of a polymer (such as the $\mathrm{n}$-paraffins in relation to polyethylene), in the usual case this is not realizable. The closest approximation to this situation which can be obtained in practice is a solid specimen of definable crystallinity in which a known orientation has been given to the chains. We will consider below various experimental methods associated with the attempt to achieve this.

To begin with, we obviously require specimens which are chemically pure, i. e., contain no other component than the polymer species. This may 
be a problem if commercial samples are used, since they often contain significant amounts of plasticizers. If the plasticizer in the polymer is known, its contribution to the spectrum can be corrected for by reference to a catalog of plasticizer spectra [KENDALL, HAMPTON, HAUSDORFF, and Pristera (88)]. It may also be possible to remove the plasticizer from the polymer by extraction with organic solvents or by means of vacuum distillation. When a film is prepared by casting from a solution, it is of course necessary to be certain that no solvent remains in the specimen. In some cases the solvent may be difficult to remove completely, and allowance for this must be made in interpreting the spectrum of the polymer.

The primary dimensional requirement on a polymer sample is that it be sufficiently thin. (It is possible to obtain reflection spectra of polymers [RoBINSON and PRICE $(787,188)$ ], in which case thin specimens are not required, but the use of this technique has thus far not proven to be as fruitful as transmission spectra, and we will not consider it here.) In the $\mathrm{NaCl}$ prism region (roughly 650 to $3500 \mathrm{~cm}^{-1}$ ) specimens as thin as $0.002 \mathrm{~mm}$ may be required in order to avoid essentially $100 \%$ absorption at some band peaks. The average thickness required in this region for most bands is usually about $0.02 \mathrm{~mm}$. Thicknesses about ten times larger are optimum for frequencies above $3500 \mathrm{~cm}^{-1}$ (the overtone and combination region) and below $650 \mathrm{~cm}^{-1}$ (the far infrared region). Samples areas down to 1 by $3 \mathrm{~mm}$ are usable [WooD (247)], and even smaller if a microspectrometer is employed [BLouT (16)].

There are several techniques for producing thin films. If the polymer is soluble, it can be dissolved and a solution of the appropriate concentration cast on the surface of glass or of mercury. When the solvent has evaporated, the film is stripped from the surface (sometimes under running water for films cast on glass), and if necessary oven dried in order to remove any remaining solvent. If the polymer "melts" without decomposing, a thin film can be obtained by heating between thin $\mathrm{AgCl}$ or mica sheets and pressing the sheets together [SANDS and TuRner (192)]. This technique is most suitable when it is feasible to examine the entire laminated assembly in the spectrometer (making use of the transparency of the $\mathrm{AgCl}$ or mica in a particular spectral region), since it is usually not easy to remove the polymer film. To obtain an isolated film, it is often possible to enclose the polymer, for example in powder form, in metal foil, press under heat, and then strip the film from the foil. Some polymers resist solution or melt techniques, and in such cases thin sections can often be obtained by use of a microtome [AMBRose and ElLiotT (5)]. Another method that has been used with success [ElliotT (51); WoOD (248)] is to grind down a thicker specimen with abrasives after having cemented the specimen to a firm support. Effectively thin specimens can, of course, be prepared by the alkali halide pressed-disk 
technique, but in such cases care must be exercised that no significant changes occur in the spectrum as a result of this preparative method [FARMER (54)].

Orientation of the polymer chains in a specimen can be produced in several ways. The simplest is to stretch the film (or fiber), after heating or swelling it if necessary. Or the film can be rolled between rollers, which can also be heated. Sometimes the rolling is done after sandwiching the material between $\mathrm{AgCl}$ sheets [Elliott, Ambrose, and Temple (53)], the $\mathrm{AgCl}$ being removed if necessary by soaking in sodium thiosulfate solution [Ambrose, Elliott, and Temple (6)]. Oriented films have also been produced by spreading out a suitably concentrated solution of the polymer with a single stroke of a razor blade [AMBROSE and ELLIOTT (4)]. It is important to note that stretching and rolling usually produce different kinds of chain orientation in a polymer film. In a stretched specimen, a particular crystallographic axis (usually the long chain axis) will tend to become aligned parallel to the stretching direction, but all orientations of the crystallites about this axis will be equally probable. This is known as uniaxial orientation. In a rolled specimen, not only is such an axis aligned with the rolling direction, but particular crystallographic planes are also oriented parallel to the plane of the film. This double orientation can in some cases affect the relative intensities of bands in the infrared spectrum, and care must also be taken in the interpretation of polarized spectra for such cases. It is desirable in such instances to verify the presence or absence of double orientation by an $\mathrm{x}$-ray diffraction study, so that the dichroic results are not misinterpreted.

When changes in the spectrum occur with crystallization of the polymer, it is desirable to be able to examine a series of polymers of varying crystallinity. Often the maximum achievable degree of crystallinity in a specimen is determined by the method of polymerization. Lower degrees of crystallinity can then be obtained by heating the specimen (usually enclosed in a cell). Sometimes a completely amorphous sample can be obtained by rapid quenching of a melted specimen. Conversely, the crystallinity of a specimen can often be improved by annealing. Such changes should be verified by x-ray diffraction. In some instances the crystallizable or non-crystallizable portion of a specimen can be removed by extraction with a solvent. This will also permit obtaining specimens with varying degrees of crystallinity. X-ray diffraction techniques exist for determining quantitatively the degree of crystallinity in a polymer [Hermans and Weidinger (75); Matthews, Peiser, and Richards (127); KrImm and Tobolsky (99)], but this is often not necessary. It may be sufficient to know whether a band increases or decreases in intensity with increasing crystallinity, the latter being qualitatively determined from $x$-ray diffraction photographs. 


\section{Methods of Spectral Analysis of High Polymers}

In order to make a complete assignment of the vibrational spectrum of a high polymer, two primary requirements must be met. First, it is necessary to be able to predict the nature of the expected spectrum. This should include information on the number of expected fundamental frequencies, their activity in the infrared and Raman spectra, the dichroic properties of such fundamentals and of overtones and combination bands, the form of each normal vibration, and, if possible, the calculated frequency of each normal vibration. Second, it is necessary to be able to correlate unambiguously the observed bands with the results of the above predictions. This implies having experimental information on the entire spectrum, both infrared and Raman, as well as polarization measurements, spectra of isotopically substituted molecules, and any other data which will aid in identifying the observed bands. While the ideal implied in the above requirements is far from having been reached, it is as a result of recent developments in many of the areas mentioned that significant progress toward complete spectral assignments for polymers has been made. In the following sections we will discuss these various methods which contribute to the spectral analysis of high polymers.

\section{A. Theoretical Methods of Analysis}

\section{Symmetry Analysis}

As was noted earlier, when a molecule possesses symmetry it is possible to determine many features of its spectrum without any additional knowledge. The extent of such spectral information is directly related to the degree of symmetry of the molecule. The technique for doing this, viz. group theory, has been utilized extensively in the spectroscopic study of small molecules, but has only recently been applied to the investigation of high polymers. We cannot develop here the foundation of this method. This has been covered in a number of books [BHAGavantam and VenKatarayudu (15); Wilson, Decuis, and Cross (245); Wigner (242)] and articles [Rosenthal, and Murphy (189); Meister, Cleveland, and Murray (129)], and applied to the analysis of the spectra of crystals [Halford (72); Hornig (79); Winston and Halford (246); Walnut (235)] and of polymers [Tobin (230); Liang, Krimm, and SutherLand (117); LIANG (110)]. We will quote the results of this theory and show how they can be applied to the analysis of high polymer spectra.

The basis of the application of group theory to the classification of the normal vibrations of a molecule lies in the fact that the potential and kinetic energies of a molecule are invariant to symmetry operations. A symmetry operation is a physical transformation of the molecule, such as reflection in a mirror plane of symmetry or rotation through $120^{\circ}$ about 
a 3-fold symmetry axis in the molecule, which brings it into a configuration totally indistinguishable from that which it had before the symmetry operation was performed. The invariance follows from the fact that, for example, the potential energy is assumed to depend only on the displacements of atoms from their equilibrium posititions, i. e., on internuclear distances, and these are not altered by a symmetry transformation. The set of symmetry operations having this common property is always found to form a group, in the mathematical sense (i. e., the product of two or more operations is equivalent to some operation in the set, the set contains an identity operation, every operation of the set has an inverse which is also in the set, and the associative law of multiplication holds between members of the set). Furthermore, the set of matrices which give the analytical transformations corresponding to the physical operations forms a group isomorphic with that of the symmetry operations, i. e., a one-to-one correspondence can be made between elements of the two groups such that the product of two elements in one group corresponds to that in the other. For instance, a $\frac{2 \pi}{\varphi}$-fold rotation axis parallel to the $z$ axis will transform the coordinates $x, y, z$ of an atom into coordinates $x^{\prime}, y^{\prime}, z^{\prime}$, where

$$
\begin{aligned}
& x^{\prime}=\quad x \cos \varphi+y \sin \varphi \\
& y^{\prime}=-x \sin \varphi+y \cos \varphi \\
& z^{\prime}=
\end{aligned}
$$

The matrix of this transformation is

$$
\left\|\begin{array}{ccc}
\cos \varphi & \sin \varphi & 0 \\
-\sin \varphi & \cos \varphi & 0 \\
0 & 0 & 1
\end{array}\right\|
$$

and analogous matrices exist for the other symmetry operations. Such a set of matrices is known as a representation of the group. Usually not only are these matrices isomorphic with the group of physical operations but component sub-matrices of these matrices also have this property. Sets of the smallest possible non-equivalent sub-matrices of such a representation are known as irreducible representations of the group. It turns out that an irreducible representation is adequately characterized for our purposes by giving only the sum of the terms along the principal diagonal instead of the complete matrix for each symmetry element. Such a sum is known as the character of the matrix, and its utility lies in the fact that for a given type of symmetry transformation its value is independent of the coordinate system used. Character tables, giving the characters of all of the irreducible representations, have been worked out for the finite number of point groups [see, for example, WiLson, Decius, 
and Cross (245)], i.e., symmetry groups all of whose operations leave one point in space fixed. Since the groups for polymers are isomorphic with some of these point groups, we can make direct use of these character tables.

Before discussing the use of such character tables, we will illustrate how the appropriate one is obtained for a given polymer structure. As an example, let us take a single planar zig-zag (infinitely long) polyethylene chain. The complete analysis requires us to use the true crystal structure, which contains two chains per unit cell [BunN (26)], but it has been

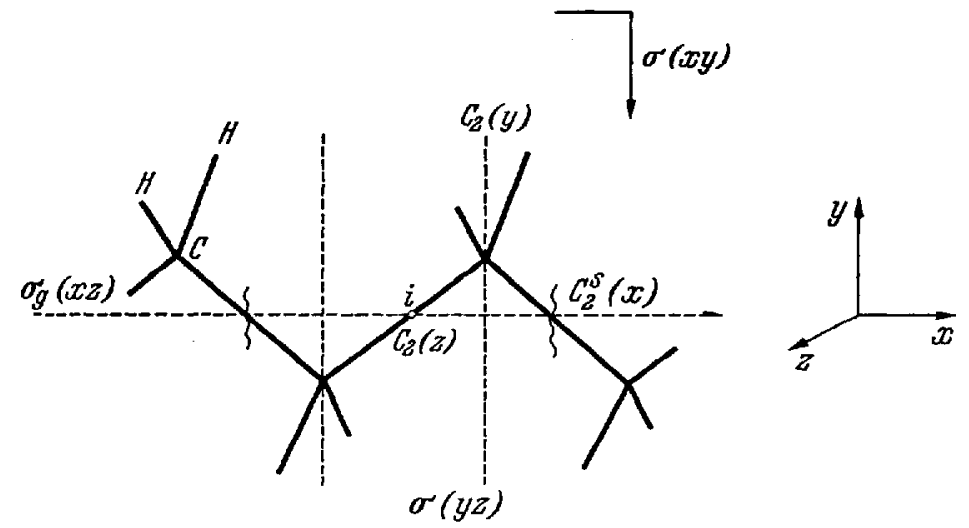

Fig. 2. Structure and symmetry elements for a planar zig-zag polyethylene chain. [KRIMM, LianG, and SUtherLAND (702)]

shown [ToBin (230); Krimm, Liang, and SUtherLand (102)] that the single chain analysis satisfactorily predicts the main features of the spectrum. The relationship between these two approaches will be considered in more detail later.

First, it is necessary to define the structure. The structure of a planar zig-zag polyethylene chain is shown in Fig. 2, together with its symmetry elements. These are: $C_{2}-$ a two-fold rotation axis, $C_{2}^{s}-\mathrm{a}$ two-fold screw axis, $i$ - a center of inversion, $\sigma-$ a mirror plane, and $\sigma_{g}-$ a glide plane. Not shown are the indentity operation, $E$, and the infinite number of translations by multiples of the repeat (or unit cell) distance along the chain axis. All of these symmetry operations, but no others, leave the configuration of the molecule unchanged.

Second, a multiplication table for the factor group is written down. The space group formed by the above symmetry elements is infinite, because of the translations. If we define the translations, which carry a point in one unit cell into the corresponding point in another unit cell, as equivalent to the identity operation, then the remaining symmetry elements form a group known as the factor, or unit cell, group. The factor 
group is a sub-group of the space group, and is always isomorphic with one of the 32 crystallographic point groups. Its significance lies in the fact that only the factor group fundamentals may be infrared or Raman active. Since factor group modes are by definition ones which are symmetric with respect to translation, this has the physical significance that only those fundamental frequencies can be active which correspond to simultaneously identical motions of equivalent atoms in all unit cells. Modes in which the phase of the motion varies with the translational coordinate cannot be active. The multiplication table for the group is obtained by forming all of the possible products of two symmetry elements. The multiplication table for a polyethylene chain is shown in Table 2.

Table 2. Multiplication Table for Polyethylene Chain Factor Group

\begin{tabular}{l|c|c|c|c|c|c|c|c}
\hline & $E$ & $C_{2}(z)$ & $C_{2}(y)$ & $C_{2}^{s}(x)$ & $i$ & $\sigma(x y)$ & $\sigma_{g}(x z)$ & $\sigma(y z)$ \\
\hline$E$ & $E$ & $C_{2}(z)$ & $C_{2}(y)$ & $C_{2}^{s}(x)$ & $i$ & $\sigma(x y)$ & $\sigma_{g}(x z)$ & $\sigma(y z)$ \\
$C_{2}(z)$ & $C_{2}(z)$ & $E$ & $C_{2}^{s}(x)$ & $C_{2}(y)$ & $\sigma(x y)$ & $i$ & $\sigma(y z)$ & $\sigma_{g}(x z)$ \\
$C_{2}(y)$ & $C_{2}(y)$ & $C_{2}^{s}(x)$ & $E$ & $C_{2}(z)$ & $\sigma_{g}(x z)$ & $\sigma(y z)$ & $i$ & $\sigma(x y)$ \\
$C_{2}^{s}(x)$ & $C_{2}^{s}(x)$ & $C_{2}(y)$ & $C_{2}(z)$ & $E$ & $\sigma(y z)$ & $\sigma_{g}(x z)$ & $\sigma(x y)$ & $i$ \\
$i$ & $i$ & $\sigma(x y)$ & $\sigma_{g}(x z)$ & $\sigma(y z)$ & $E$ & $C_{2}(z)$ & $C_{2}(y)$ & $C_{2}^{s}(x)$ \\
$\sigma(x y)$ & $\sigma(x y)$ & $i$ & $\sigma(y z)$ & $\sigma_{g}(x z)$ & $C_{2}(z)$ & $E$ & $C_{2}^{s}(x)$ & $C_{2}(y)$ \\
$\sigma_{g}(x z)$ & $\sigma_{g}(x z)$ & $\sigma(y z)$ & $i$ & $\sigma(x y)$ & $C_{2}(y)$ & $C_{2}^{s}(x)$ & $E$ & $C_{2}(z)$ \\
$\sigma(y z)$ & $\sigma(y z)$ & $\sigma_{g}(x z)$ & $\sigma(x y)$ & $i$ & $C_{2}^{s}(x)$ & $C_{2}(y)$ & $C_{2}(z)$ & $E$ \\
& $\sigma$ & & & & & &
\end{tabular}

Third, the point group isomorphic with the factor group is determined, and its character table written down. By examination of a list of the various point groups and their symmetry elements [e. g., Bhagavantam and Venkatarayudu (15), p. 124] it can be ascertained that only point group $D_{2 h}$ is isomorphic with the polyethylene chain factor group, i. e., a one-to-one correspondence can be made between the symmetry elements of the two groups such that their multiplication tables are identical. The character table can be readily obtained [WILson, Decius, and CRoss (245)], and it is reproduced in the upper left hand portion of Table 3. There are eight irreducible representations, usually called symmetry species, which in the present case consist of onefold matrices whose element is +1 or -1 and therefore whose character is also +1 or -1 . The matrices are of this form because all of the normal modes are non-degenerate, and therefore since a symmetry operation performed on a normal coordinate must leave the potential energy unchanged, i. e.,

$$
V=\frac{1}{2} \sum_{i=1}^{3 N} \lambda_{i} Q_{i}^{i}=\frac{1}{2} \sum_{i=1}^{3 N} \lambda_{i}\left(R Q_{i}\right)^{2}
$$

it follows that $R Q_{k}= \pm Q_{k}$. The characters therefore describe the behavior of a normal mode with respect to the various symmetry operations, i. e., whether it is symmetric or antisymmetric. Species are designated 
by $A$ or $B$ according to whether they are symmetric or antisymmetric with respect to rotation axes, and by $g$ or $u$ according to whether they are symmetric or antisymmetric with respect to the center of symmetry.

Table 3. Character Table and Selection Rules for Polyethylene Chain

\begin{tabular}{|c|c|c|c|c|c|c|c|c|c|c|c|c|c|c|c|}
\hline$D_{2 h}$ & $E$ & $C_{2}(z)$ & $C_{2}(y)$ & $C_{2}^{s}(x)$ & $i$ & $\sigma(x y)$ & $\sigma_{g}(x z)$ & $|\sigma(y z)|$ & $n_{i}$ & $T$ & $T^{\prime}$ & $\boldsymbol{K}^{\prime}$ & $n_{i}^{\prime}$ & $I K$ & $R$ \\
\hline 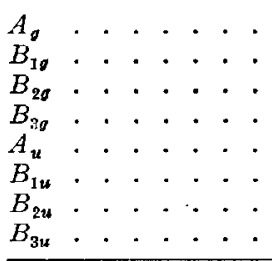 & \begin{tabular}{l|}
1 \\
1 \\
1 \\
1 \\
1 \\
1 \\
1 \\
1 \\
1
\end{tabular} & \begin{tabular}{|r|}
1 \\
1 \\
-1 \\
-1 \\
1 \\
1 \\
-1 \\
-1 \\
\end{tabular} & $\begin{array}{r}1 \\
-1 \\
1 \\
-1 \\
1 \\
-1 \\
1 \\
-1\end{array}$ & $\begin{array}{r}1 \\
-1 \\
-1 \\
1 \\
1 \\
-1 \\
-1 \\
1\end{array}$ & $\begin{array}{r}1 \\
1 \\
1 \\
1 \\
-1 \\
-1 \\
-1 \\
-1\end{array}$ & $\begin{array}{r}1 \\
1 \\
-1 \\
-1 \\
-1 \\
-1 \\
1 \\
1\end{array}$ & $\begin{array}{r}1 \\
-1 \\
1 \\
-1 \\
-1 \\
1 \\
-1 \\
1\end{array}$ & $\begin{array}{r}1 \\
-1 \\
-1 \\
1 \\
-1 \\
1 \\
1 \\
-1\end{array}$ & $\begin{array}{l}3 \\
2 \\
1 \\
3 \\
1 \\
3 \\
3 \\
2 \\
\end{array}$ & $\begin{array}{c}0 \\
0 \\
0 \\
0 \\
0 \\
T_{z} \\
T_{y} \\
T_{x} \\
\end{array}$ & $\begin{array}{c}1 \\
1 \\
0 \\
R_{x} \\
0 \\
0 \\
0 \\
0 \\
\end{array}$ & $\begin{array}{l}0 \\
1 \\
1 \\
1 \\
1 \\
1 \\
0 \\
1\end{array}$ & $\begin{array}{l}2 \\
0 \\
0 \\
1 \\
0 \\
1 \\
2 \\
0\end{array}$ & $\begin{array}{l}f \\
f \\
f \\
f \\
f \\
a \\
a \\
a\end{array}$ & $\begin{array}{l}P \\
D \\
D \\
D \\
f \\
f \\
f \\
f\end{array}$ \\
\hline $\begin{array}{l}U_{R} \\
\varphi_{1} \\
\pm 1+2 \cos \varphi \\
U_{R}( \pm 1+2 \cos \varphi)\end{array}$ & $\begin{array}{c}6 \\
0^{\circ} \\
3 \\
18\end{array}$ & $\begin{array}{c}0 \\
180^{\circ} \\
-1 \\
0\end{array}$ & $\begin{array}{r}2 \\
180^{\circ} \\
-1 \\
-2\end{array}$ & $\left|\begin{array}{c}0 \\
180^{\circ} \\
-1 \\
0\end{array}\right|$ & $\begin{array}{c}0 \\
180^{\circ} \\
-3 \\
0\end{array}$ & $\begin{array}{r}2 \\
0^{\circ} \\
1 \\
2\end{array}$ & $\begin{array}{r}0 \\
0^{\circ} \\
1 \\
0\end{array}$ & $\begin{array}{r}6 \\
0^{\circ} \\
1 \\
6\end{array}$ & & & & & & & \\
\hline $\begin{array}{l}U_{R}(s) \cdot . \\
\left.\quad U_{R}(s)-1\right] \\
\quad( \pm 1+2 \cos \varphi) .\end{array}$ & $\begin{array}{l}2 \\
3\end{array}$ & $\begin{array}{l}0 \\
1\end{array}$ & $\begin{array}{r}2 \\
-1\end{array}$ & 0 & $\begin{array}{l}0 \\
3\end{array}$ & $\begin{array}{l}2 \\
1\end{array}$ & $\begin{array}{r}0 \\
-1\end{array}$ & $\begin{array}{l}2 \\
1\end{array}$ & & & & & & & \\
\hline $\begin{array}{l}U_{R}(s-v) \\
{\left[U_{R}(s-v)\right]} \\
\quad(1 \pm 2 \cos \varphi) . .\end{array}$ & $\begin{array}{l}2 \\
6\end{array}$ & 0 & $\begin{array}{r}2 \\
-2\end{array}$ & 0 & 0 & $\begin{array}{r}2 \\
-2\end{array}$ & 0 & $\begin{array}{r}2 \\
-2\end{array}$ & & & & & & & \\
\hline $\begin{array}{l}2 \cos \varphi \\
\quad( \pm 1+2 \cos \varphi) .\end{array}$ & 6 & 2 & 2 & 2 & 6 & 2 & 2 & 2 & & & & & & & \\
\hline
\end{tabular}

Having the character table, we will now show how to determine the number of modes associated with each symmetry species and their activity. We will quote the relevant formulae [BHAGAvantam and VENKATARAYUdU (15)] and apply them to the example of the polyethylene chain. The total number of normal modes (including translations and rotations) under a given species is given by

$$
n_{i}=\frac{1}{N} \sum_{n} U_{R}\left( \pm 1+2 \cos \varphi_{R}\right) \chi_{i}(R)
$$

where $N$ is the order of the group; $U_{R}$ is the number of atoms that remain invariant under the symmetry operation $R$, recalling that a translation is equivalent to the identity operation; the plus or minus sign is to be used according as $R$ is a pure rotation through $\varphi$ or a rotation through $\varphi$ followed by a reflection in a plane perpendicular to the axis; $\chi_{i}(R)$ is the character of the symmetry element in the $i^{\text {th }}$ species; and the summation extends over all of the symmetry elements. These various quantities are listed in Table 3 and have been used to obtain the numbers in the column 
under $n_{i}$. For example:

$$
\begin{aligned}
n_{A_{g}}=\frac{1}{8}[(18)(1) & +(0)(1)+(-2)(1)+(0)(1)+(0)(1)+(2)(1)+(0)(1)+ \\
& +(6)(1)]=3 \\
n_{B_{1} g}=\frac{1}{8}[(18)(1) & +(0)(1)+(-2)(-1)+(0)(-1)+(0)(1)+(2)(1)+ \\
& +(0)(-1)+(6)(-1)]=2 .
\end{aligned}
$$

The total number of pure translations under a given species is given by

$$
n_{i}(T)=\frac{1}{N} \sum_{R}\left( \pm 1+2 \cos \varphi_{R}\right) \chi_{i}(R)
$$

where the symbols have the same meaning as before. By applying this formula to the quantities tabulated in Table 3 we get the results in the column under $T$. The coordinate axis along which the translation occurs is then clear from the symmetry of the species.

The difference between $n_{i}$ and $n_{i}(T)$ represents the number of internal vibrational modes (except for rotations, one of which is possible in an infinite isolated chain, viz., that about the axis). It is possible, however, to subdivide these $3 p-4$ internal modes of a linear chain (where $p$ is the number of atoms in the repeat unit) into various categories. If we can meaningfully divide the $p$ atoms in the repeat unit into groups such that the forces between groups are generally small compared to the forces between atoms within a single group, then it is possible to determine the number of translatory and rotatory types of oscillations in which the groups move as units with respect to each other. For example, in the polyethylene chain the $\mathrm{CH}_{2}$ unit qualifies as such a group. If $U_{R}(s)$ is the number of such groups that remain invariant for the symmetry operation $R$, then the number of translatory type oscillations of these groups with respect to each other is given by

$$
n_{i}\left(T^{\prime}\right)=\frac{1}{N} \sum_{R}\left[U_{R}(s)-1\right]\left( \pm 1+2 \cos \varphi_{R}\right) \chi_{i}(R)
$$

and the number of rotatory type oscillations is given by

$$
n_{i}\left(R^{\prime}\right)=\frac{1}{N} \sum_{R}\left[U_{R}(s-v)\right]\left(1 \pm 2 \cos \varphi_{R}\right) \chi_{i}(R)
$$

In the last equation, $v$ represents the number of groups which consist of a single atom only, so that we are only concerned with the $s-v$ groups that are made up of more than one atom; $U_{R}(s-v)$ is the number of such groups which remain invariant under the operation $R$. Applying these results to the polyethylene chain, we get the numbers in the columns under $T^{\prime}$ and $R^{\prime}$ (note that $v=0$ for our case). One of the $T^{\prime}$ modes is the rotation about the chain axis. The remaining modes must be the internal 
oscillations within the group, and these are given (by subtraction) in the column under $n_{i}^{\prime}$.

We have therefore been able to determine the distribution of the normal vibrations among the various symmetry species. Because of the small number often associated with each species, and because the characters give us the behavior with respect to the symmetry operations, it is usually quite easy to determine by inspection the general nature of the vibrations. Strictly speaking, we will be able to deduce in this way possible symmetry coordinates rather than the normal coordinates. Only if a species contains one mode will the two be the same. Otherwise the true normal coordinates in a given species will consist of combinations of the symmetry coordinates of that species, the coefficients depending on the force constants. Nevertheless, the symmetry coordinates represent a significant insight into the vibrations of the chain, and in fact are usually fairly close to the true normal coordinates. A set of such modes for the polyethylene chain is shown in Fig. 3.

We consider next the activity of the fundamentals. Modes under a given species can be infrared active only if the quantity

$$
n_{i}(M)=\frac{1}{N} \sum_{R}\left( \pm 1+2 \cos \varphi_{R}\right) \chi_{i}(R)
$$

is different from zero. We see that this equation is the same as that, (29), which gives the translations, so that possible activity in the infrared has already been determined. The reason for this is that we are interested here in the transformation properties of the dipole moment, which transforms like a vector just as does a translation. In fact the polarization properties of infrared active modes in a given species will be the same as the translation direction associated with that species. Activity in the Raman spectrum is determined by the transformation properties of the polarizability, which is a tensor. In this case, modes under a given species can be Raman active if the quantity

$$
n_{i}(\alpha)=\frac{1}{N} \sum_{R} 2 \cos \varphi_{R}\left( \pm 1+2 \cos \varphi_{R}\right) \chi_{i}(R)
$$

is different from zero. The results obtained for a polyethylene chain are listed in Table 3 in the column under $I R$ and $R$, where $f$ means forbidden, $a$ means active, and $P$ and $D$ signify polarized and depolarized Raman lines respectively. Of course, these results provide only a necessary, not a sufficient, condition for the appearance of a band in absorption or scattering, since the intensity is determined by additional factors.

The preceding discussion has been concerned with the properties of fundamental frequencies. Bands of significant intensity which are due to combinations and overtones do appear, however, in polymer spectra, and 

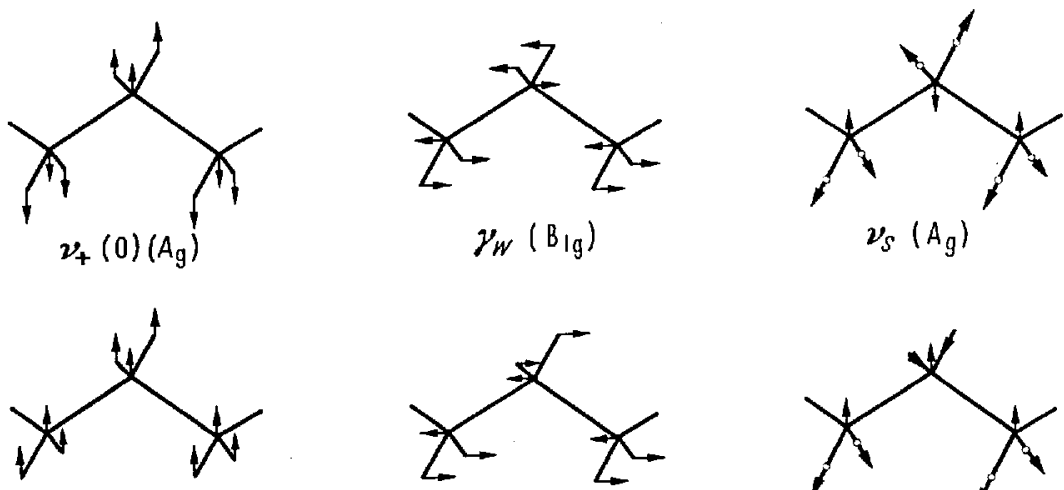

$T_{y}\left(B_{2 u}\right)$

$\gamma_{w}\left(B_{3 u}\right)$
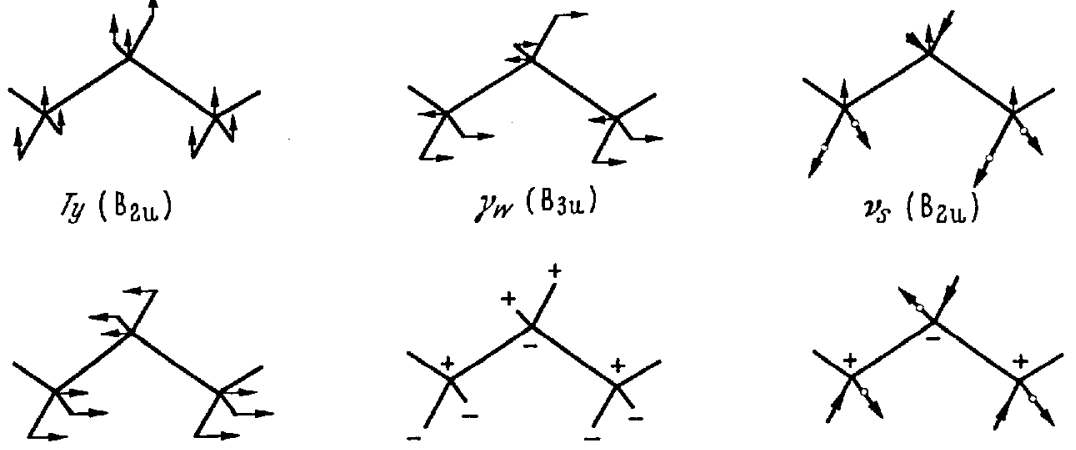

$\nu_{+}(x)(B, g)$

$\gamma_{r}\left(B_{3 g}\right)$

$$
\nu_{a}\left(B_{3 g}\right)
$$
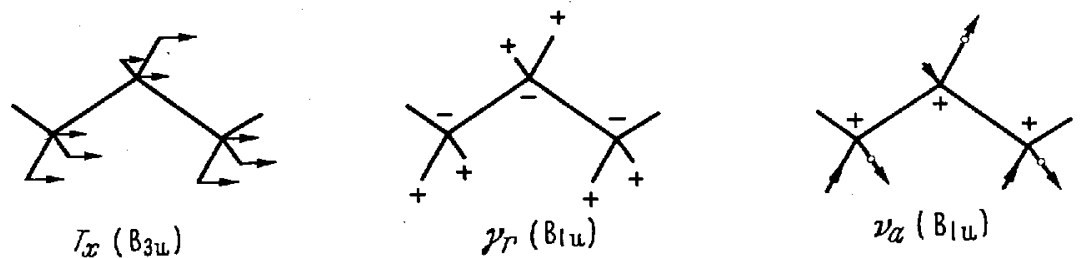

$T_{x}\left(B_{3 L}\right)$

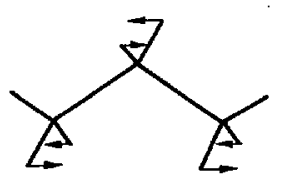

$\nu_{a}\left(B_{\mid u}\right)$
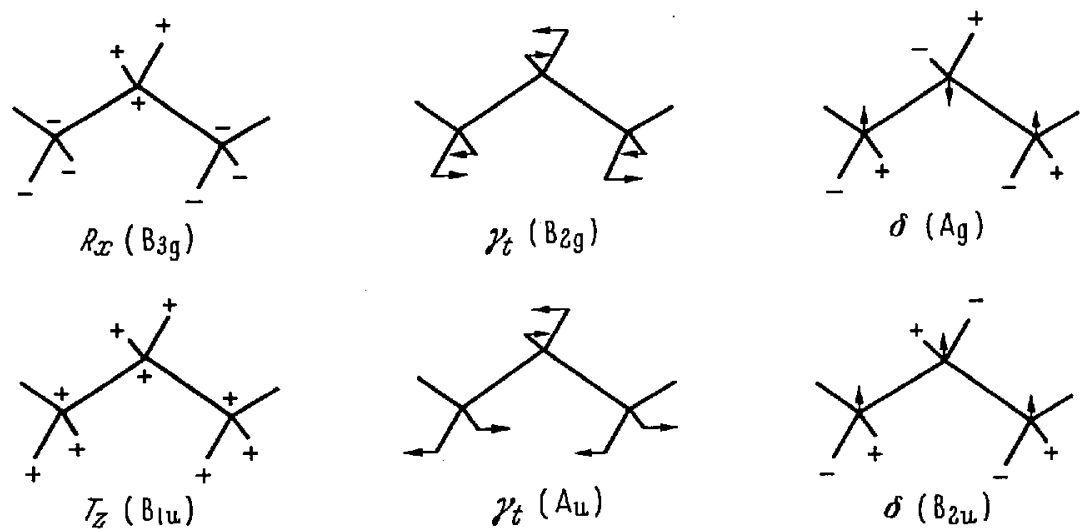

Fig. 3. Symmetry modes of a single polyethylene chain. [KRImm, Liang, and Sutherdand (102)] 
their identification is often an important part of the analysis, particularly since it helps establish the assignments of the fundamentals. The rule for determining the activity of non-degenerate combinations and overtones of factor group fundamentals is simple [for the overtones of degenerate vibrations see, e.g., Meister, Clevel.and, and Murray (129)]: multiply the characters of the irreducible representations of the species involved; the species of the resultant representation gives the activity of the combination or overtone. For example, in the case of the polyethylene chain all overtones belong to the $A_{g}$ species and are therefore infrared inactive; the combination between a $B_{3 g}$ and an $A_{u}$ belongs to the $B_{3 u}$ representation, and is therefore infrared active. The theory indicates, however, that not only can such factor group combinations be active, but combinations and overtones of non-factor group modes can also be active, in distinction to the fundamentals [WINSTON and HALFORD (246)]. The only requirement in the latter case is that the combined representation should be totally symmetric with respect to the translations. The consequence of this condition is that many more binary combinations are permitted than would be allowed by the factor group analysis. Actually, no such combinations have been unambiguously identified in polymer spectra, and in fact it appears that almost all of the bands observed for polyethylene can be interpreted in terms of factor group fundamentals [NIELSEN and WoolletT (160)]. We will therefore assume that the major number of combination bands are to be interpreted in terms of factor group fundamentals.

We wish now to comment on the relationship between the analysis based on a single chain, i. e., on the one-dimensional space group, and the complete one based on the three-dimensional crystal structure. If the unit cell contains only one chain, the one-dimensional analysis gives the correct selection rules. If there are two, or more, chains per unit cell, then two kinds of differences can occur. Internal group motions which were inactive for a single chain can be allowed in the crystal. This is true of the $\mathrm{CH}_{2}$ twisting mode in polyethylene [KRIMM, LIANG, and SuTHERLAND (102)]. More important, if interactions between molecules [the $V_{j k}^{\prime \prime}$ term in equation (20)] are important, the polarization properties deduced from the single chain analysis may be incomplete. In crystalline polyethylene, for example, modes which the single chain analysis indicates are polarized perpendicular to the chain axis not only have this characteristic but are polarized specifically along one or other of the crystallographic axes perpendicular to the chain direction [KRIMM (93)]. Polyethylene is the only polymer for which such interactions have been unambiguously demonstrated, so that in general the single chain analysis should be satisfactory.

In summary, a symmetry analysis of a polymer permits a classification of the normal modes which can be helpful in identifying them. For 
example, for polyethylene we know from Table 3 that there is a single $B_{3 u}$ infrared active mode which can be identified by its polarization parallel to the chain $(x)$ axis. Unfortunately, symmetry analysis is useful in direct proportion to the degree of symmetry of the molecule, and most polymer molecules have relatively little symmetry, even in the crystalline state. In addition, a significant portion of most polymers is amorphous, and we might expect that no selection rules would exist in this case. Actually, because of the general validity of group frequencies, it is often possible to analyze to a good approximation the vibrational modes of portions of a molecule in terms of the local symmetry. Thus, the benzene ring modes in polystyrene can be classified and successfully analyzed on the basis of an assumed monosubstituted benzene ring of symmetry $C_{2 v}$ [LIANG and KrImM (114)]. The same is true of the phenyl group modes in polyethylene terephthalate [LIANG and Krimm (115)]. This means that symmetry arguments can generally be applied to the preliminary analysis of the spectrum expected from a polymer. Since these arguments in turn rest on the structure of the polymer, assumed or known, the agreement between the predicted and observed spectrum is one means of obtaining information on the polymer structure.

\section{Normal Vibration Analysis}

A more detailed and complete understanding of the structure and potential function of a molecule requires that we be able to predict correctly the numerical values of its normal vibrational frequencies. These are determined by the kind of theoretical analysis outlined in Section I. 1., which we have seen invokes knowledge of the mass distribution and force field of the molecule. If we can show that the experimentally observed bands correspond not only qualitatively but also quantitatively to those predicted, we have a secure basis for claiming detailed knowledge about a molecule.

Such normal vibration analyses have been applied to the spectra of macromolecules to only a limited extent. In the first place, the only structure which has been analyzed in detail is that of the planar zig-zag chain of $\mathrm{CH}_{2}$ groups, i.e., polyethylene. Neither substituted planar zig-zag chains nor the helical chain structures characteristic of many polymers [BUNN and Holmes (28)] have been submitted to such a theoretical analysis. In the second place, even for the case of polyethylene the answers are not in all instances unambiguous. Different assumptions as to the nature of the force field, and lack of knowledge of some of the force constants, has led to varying predictions of band positions in the observed spectrum. For the identification of certain modes, viz., those which retain the characteristics of separable group frequencies, such an analysis is not of primary importance, but for knowledge of skeletal frequencies and of interactions 
between different vibrations a normal vibration analysis is vital. We will discuss briefly the results of this kind of analysis for the polyethylene chain.

Two approaches have been taken in computing the normal vibration frequencies of polyethylene. One has been concerned only with the skeletal vibrations of the zig-zag chain, both finite and infinite [BARTHOlomé and Teller, (11); Kassel (84); Kirkwood (91); Pitzer (174); Parodi (166); Ahonen (3); Barriol and Chapelle (9); Mizushima and Simanouti (138); Brown, Sheppard, and Simpson (22); Liang, Sutherland, and Krimm (118); Liang, Krimm, and SutherlaNo (177), Theimer (226)]; that is, the $\mathrm{CH}_{2}$ group is considered to be a point mass. In the other approach an attempt has been made to compute all of the normal vibrations, hydrogen as well as skeletal [WHITCOMB, NiELSEN, and Thomas (240); Stepanov (209); Simanouti (199); Simanouti and Mizushima (200); Kellner (87); Barrow (10); Primas and Günthard $(779,180)$; Person and Pimentel (168); Theimer (227)]. The results of the latter calculations will be discussed in more detail when we discuss the spectrum of polyethylene (Section IV. A.). At present we will consider the problem of the skeletal frequencies of the planar zig-zag chain, since the results are of more general applicability to other high polymers.

The most satisfactory treatment of this problem seems to be that originated by KIRKWOOD [KIRKWOOD (91)], and later applied more specifically to the infinite polymer chain [LIANG, SUTHERLAND, and KRIMM (118); LIANG, KRIMM, and SUTHERLAND (177)]. In this treatment the $\mathrm{CH}_{2}$ groups are replaced by point masses, arranged in a planar zig-zag configuration with tetrahedral angle between the neighboring bonds. In this approximation, interactions between the skeletal vibrations and $\mathrm{CH}_{2}$ deformation vibrations are neglected, although such interaction does exist with the $\mathrm{CH}_{2}$ wagging mode [Primas and GüNTHard (180); Theimer $(227)]$, but this does not seem to affect the frequencies seriously. With a valence force field, the potential contains terms involving the stretching of the $\mathrm{C}-\mathrm{C}$ bond and the bending of the $\mathrm{CCC}$ angle. When the equations of motion of the $n^{\text {th }}$ element are set up, as described in Section I. 1., the secular equation has solutions given by

where

$$
\lambda_{ \pm}=\lambda_{0} \pm\left(\lambda_{0}^{2}-\lambda_{1}^{2}\right)^{1 / 2}
$$

$$
\begin{aligned}
\lambda_{0} & =\frac{k_{c}}{m}\left(1-\frac{1}{3} \cos \varphi\right)+\frac{2 k_{\alpha}}{m}(1+\cos \varphi)\left(1+\frac{1}{3} \cos \varphi\right) \\
\lambda_{1}^{2} & =\frac{8 k_{c} k_{\alpha}}{m^{2}}(1+\cos \varphi) \sin ^{2} \varphi \\
\lambda_{ \pm}(\varphi) & =4 \pi^{2} \nu_{ \pm}^{2}(\varphi) .
\end{aligned}
$$


In these equations, $m$ is the mass of an element (a $\mathrm{CH}_{2}$ group), $k_{\mathrm{c}}$ is the force constant for stretching of a $\mathrm{C}-\mathrm{C}$ bond, $k_{\alpha}$ is the force constant for bending of the CCC angle, and $\varphi$ is the phase difference between the motions of adjacent elements of the chain. Since we are interested only in the factor group modes, i.e., those in which the vibrations of corresponding elements in neighboring unit cells are in phase, we require that

$$
\varphi=\frac{2 \pi r}{q} \quad r=0,1,2, \ldots, q-1
$$

where $q$ is the number of elements in the unit cell. For a planar zig-zag chain $q=2$, and therefore the only potentially active modes are those for which $\varphi=0$ and $\varphi=\pi$. Studies on small hydrocarbons [AHONEN (3); RASMUSSEN (184)] suggest that reasonable values for the force constants are $k_{c}=4.0 \times 10^{5}$ dynes $/ \mathrm{cm}$ and $k_{\alpha}=0.38 \times 10^{5} \mathrm{dynes} / \mathrm{cm}$. With these values we find from equations $(33)-(36)$ that

$$
\nu_{+}(0)=1065 \mathrm{~cm}^{-1} \text { and } \nu_{+}(\pi)=1135 \mathrm{~cm}^{-1}
$$

[These values differ slightly from those previously given by LIANG, KRIMM, and Sutherland (177).] These modes, which are shown in Fig. 3 (of symmetry species $A_{g}$ and $B_{1 g}$ respectively), are expected to be Raman active and infrared inactive (see Table 3), although weak activity in the infrared may result from boundary effects [KELLNER (87); THErmer, (226)]. It is interesting to note that the Raman spectrum of polyethylene (as well as that of hydrocarbons) exhibits moderately strong bands at $1061 \mathrm{~cm}^{-1}$ and $1131 \mathrm{~cm}^{-1}$, in good agreement with the predicted values. Earlier assignments [Mizushima and Simanouti (138); Brown, ShepPARd, and Simpson (22); Kellner (87); Barrow (10); Person and Pimentel (168)] of one of the sleketal modes to a band at about $890 \mathrm{~cm}^{-1}$ do not seem to be tenable. This band is most likely a $\mathrm{CH}_{3}$ mode, as indicated by its shift on deuteration in the infrared spectra of $n$-octane [Pimentel and Klemperer (172)] and higher paraffins [KRImM, Liang, and Sutherland (102)], and its decrease in intensity in the Raman spectrum with increase in chain length [NIELSEN and Woollet (160)].

In cases where $q=4$, e. g., when the positions of substituents on the chain increase the true identity period to four elements, we can show that two other skeletal modes can become potentially active. This of course assumes that the substituents can be neglected and the previous treatment used, an unlikely assumption. Nevertheless the results may serve as crude guides to the location of other possible skeletal frequencies for such polymers. Application of equations (34)-(37) shows that these modes are expected at

$$
\nu_{+}\left(\frac{\pi}{2}\right)=983 \mathrm{~cm}^{-1} \text { and } \quad \nu_{-}\left(\frac{\pi}{2}\right)=430 \mathrm{~cm}^{-1} .
$$


In addition, an out-of-plane skeletal mode can be active whose frequency is given by [PITZER (174)]

$$
\lambda=\frac{k_{3}}{m}(4-2 \cos \varphi-4 \cos 2 \varphi+2 \cos 3 \varphi)
$$

where $k_{3}$ is the force constant for rotation about the $\mathrm{C}-\mathrm{C}$ bond. The value of this force constant is not well known, but on the assumption that $k_{3}=0.02 \times 10^{5}$ dynes/cm [THEIMER (226)] we find from equation (38) that

$$
\nu\left(\frac{\pi}{2}\right)=140 \mathrm{~cm}^{-1}
$$

This frequency may well range up to about $200 \mathrm{~cm}^{-1}$ [LIANG, KRIMM, arid SUTHERLAND (177)]. It should be noted that $\nu_{+}\left(\frac{\pi}{2}\right), \nu_{-}\left(\frac{\pi}{2}\right)$, and $\nu\left(\frac{\pi}{2}\right)$ are doubly degenerate, which arises from our taking two identical unit cells of the polyethylene chain for the repeat unit. If the cells are distinguishable this degeneracy is split.

Summarizing, we may say that the normal vibration analysis permits a good understanding of the skeletal modes (and hydrogen modes, as we shall see later) of the polyethylene chain. Extension of such calculations to other polymers is needed.

\section{B. Experimental Methods of Analysis}

\section{Far Infrared and Raman Spectra}

We have already remarked (Section II. 1.) on the desirability of having spectral data in the low frequency region, both infrared and Raman. It is necessary here only to re-emphasize the point that a complete spectral assignment is at best difficult in the absence of complete spectral data, and that the region below about $650 \mathrm{~cm}^{-1}$ contains a significant portion of the data in the case of many polymers. Here are found bondstretching vibrations which involve heavy atoms (e. g., $\mathrm{C}-\mathrm{Cl}$ stretching modes), and almost all of the deformation modes assiocated with atoms other than hydrogen, as well as skeletal deformation modes. In some cases, such as for example polytetrafluoroethylene [LIANG and KRIMM (111)], the majority of the absorption bands in the infrared spectrum are located in this region. It is therefore clear that a reliable interpretation of the complete vibrational spectrum cannot be achieved without the availability of spectral data in this region. In many cases infrared data alone will be adequate, but in others this is not so. For example, a glance at Table 3 will show that in the case of polyethylene some modes are infrared active and Raman inactive while for others the reverse is true. This mutual exclusion rule holds for all molecules possessing a center of symmetry, and it implies that the infrared spectrum will provide information on only part of the vibrational modes of the molecule. The ideal 
situation would be to have both infrared and Raman data over the entire spectral range. Raman spectra of polymers are often difficult to obtain, although some of these difficulties are beginning to be surmounted [ToBIN (231)]. It is to be hoped that future studies of high polymer spectra will include more experimental data at low frequencies than has been the case in the past.

\section{Deuteration Studies}

Normal vibration calculations, if based on a correct structure and correct potential field, would supposedly permit a unique correlation to be made between predicted and observed absorption bands. In most cases this ideal situation is far from being achieved in the study of high polymer spectra. More usually the structure and force field are to some extent unknown, or normal mode calculations are not available, so that other methods must be used in order to establish the origin of bands in the spectrum. Even if complete calculations were available it would be desirable to check their predictions by means other than a comparison of observed and predicted frequency values. One method of doing so is by studying isotopically substituted molecules, and the most useful case is that in which deuterium is substituted for hydrogen.

When such an isotopic substitution is made in a molecule, the forces between the atoms remain unchanged, only the masses of the hydrogen atoms changing in magnitude. The result is that those normal modes which involve primarily the motions of hydrogen atoms will be lowered in frequency upon deuterium substitution. Since the force constants remain unaltered, the frequency will be lowered by approximately $\sqrt{2}$, in no case by more than this [HALVERSON (74)]. Thus, observation of those bands which shift by the above ratio as a result of deuteration provides a means for identifying hydrogen modes. This can be especially important when several bands of different origin occur in the same region of the spectrum and the group frequency approach is not adequate to identify the bands uniquely. Furthermore, if a molecule contains different kinds of hydrogen atoms (e. g., $\mathrm{OH}, \mathrm{CH}_{2}, \mathrm{CH}_{3}$ ), and it is possible to deuterate these selectively and individually, then a more detailed identification can be made. Since hydrogen atoms usually comprise a significant fraction of the total number of atoms in a polymer molecule, it is clear that the isotopic substitution technique provides information on a significant number of bands in the spectrum. Finally, in cases where interaction occurs between hydrogen modes and other modes, so that spectral bands have in fact a complex origin, such interaction will usually disappear upon deuterium substitution, thus indicating its presence in the original molecule. This is true of both intramolecular interactions [KRIMM, LIANG, and SUTHERLAND (103)] and intermolecular interactions [KRIMM, LIANG, and SUTHERLAND (102)]. 
Although deuteration is a technique of major importance in labeling hydrogen modes in the spectrum, it would be of even greater value if it made possible the assignment of particular types of vibrations. That is, we would like not only to use the disappearance of a band on deuteration as an indication of its origin in some hydrogen mode, but to use the magnitude of its shift in order to specify the kind of vibration involved (for example, symmetric stretching, bending, etc. in the case of a $\mathrm{CH}_{2}$ group). The only exact rule which applies to such cases, viz., the TellerRedlich product rule [HeRzBerg (76)] unfortunately holds only for the product of all frequencies in a symmetry species. Since polymer molecules usually exhibit low symmetry, and therefore each symmetry species contains many frequencies, this rule is not very helpful. What would be desirable is a rule, even an approximate one, which applies to individual frequencies. We indicate below the derivation of a simple approximate rule of this nature which seems to apply quite well to the case of hydrogen-deuterium substitution [KRIMm (97)]. Its form seems to be equivalent, though simpler, than one proposed by EDGELL (50).

It can be shown [Teller (225), p. $124 \mathrm{ff}$; BeRNSTEIN (14)] that the differential frequency shift on isotopic substitution is given by

$$
\frac{d \lambda_{k}}{\lambda_{k}^{0}}=-\frac{\sum_{i} d T_{i}}{T}
$$

where $\lambda_{k}^{0}=4 \pi^{2} \nu_{k}^{02}, T=\sum_{j=1}^{N} m_{j} \dot{q}_{j}^{2}=$ total kinetic energy associated with the $k^{\text {th }}$ vibration, and $\sum_{i} d T_{i}=\sum_{i} d m_{i} \dot{q}_{i}^{2}$ is the differential change in kinetic energy associated with the substituted atoms. If only one kind of atom is substituted, viz., hydrogen in the present case, then equation (40) provides an exact expression for the derivative of $\lambda_{k}$ with respect to $m_{i}$ :

$$
\frac{d \lambda_{k}}{d m_{i}}=-\lambda_{k}^{0} \cdot \frac{\sum_{i} \dot{q}_{i}^{2}}{T} .
$$

If it were possible to find a general expression for $\lambda_{k}$ as a function of $m_{i}$, then equation (41) might be used to obtain the finite change in frequency for the $k^{\text {th }}$ mode, $\Delta \lambda_{k}$, associated with a finite change in mass, $\Delta m_{i}$. No exact expression exists which is valid for all $\lambda_{k}\left(m_{i}\right)$, but an examination of the general equations for the frequencies of small molecules [HERZBERG (76)] reveals that a relationship of the form

$$
\lambda_{k}=a_{1}+\frac{a_{2}}{m_{i}}
$$

where $a_{1}$ and $a_{2}$ are constants is satisfied in most cases to a very good approximation. If we consider cases in which the symmetry of the molecule (or group) is not changed by isotopic substitution, we expect this 
equation to be valid for the isotopic molecule to the same approximation. Utilizing equation (42), it is possible to show that

$$
\frac{\Delta \lambda_{k}}{\Delta m_{i}} \equiv \frac{\lambda_{k}-\lambda_{k}^{0}}{m_{i}-m_{i}^{0}}=\frac{1}{\varrho}\left(\frac{d \lambda_{k}}{d m_{i}}\right)
$$

where $\varrho=m_{i} / m_{i}^{0}$ is the ratio of the isotopic to the normal mass $(\varrho=2$ in the present case). By substituting (41) in (43) one finds that

$$
\frac{\lambda_{k}}{\lambda_{k}^{0}}=1-\frac{\sum \Delta T_{i}}{\varrho T}
$$

where $\sum_{i} \Delta T_{i}=\sum_{i} \Delta m_{i} \dot{q}_{i}^{2}$ is the change in kinetic energy which results from the replacement of hydrogen by deuterium, the amplitude of motion being that in the original molecule. The ratio of the zero-order frequencies is of course given by the square root of equation (44). (The zero-order frequencies differ from the observed fundamental frequencies by the anharmonicity constants [HERzBERG (76)]. In some cases these differences are significantly large.)

In order to apply equation (44) to a specific vibration in a given molecule it is necessary to know the relative amplitudes of motion of the atoms, i. e., it is necessary to know the normal coordinate. Since in most cases this is not known, it would seem difficult to make use of this isotope rule. In many cases, however, the normal coordinate is quite closely approximated by a suitably chosen symmetry coordinate. Since the latter is usually determined quite readily, and since the frequencies are stationary with respect to small changes in the normal coordinates [BERNSTEIN (14)], it can be expected that equation (44) will apply to a good approximation. This of course depends on the chosen symmetry coordinate being reasonably close to the actual normal coordinate.

Some examples of the application of equation (44) are shown in Table 4. Two small molecules, $\mathrm{H}_{2} \mathrm{O}$ and $\mathrm{NH}_{3}$, and several small hydrogencontaining groups which occur in polymer molecules, $\mathrm{CH}, \mathrm{CH}_{2}$, and $\mathrm{CH}_{3}$, are considered. In all cases (except the $\mathrm{CH}$ bend) the symmetry coordinate used was of the typical valence force field type (in the case of the $\mathrm{CH}$ bend the coordinate used was a superposition of two coordinates chosen so as to give agreement with the $\mathrm{CHCl}_{3}$ frequency). For example, the coordinates used for $\mathrm{H}_{2} \mathrm{O}$ and $\mathrm{CH}_{2}$ were those shown in Fig. 1, in which atoms move along and perpendicular to bonds. The same was true for $\mathrm{NH}_{3}, \mathrm{CH}_{3}$, and $\mathrm{CH}$ (the $\mathrm{CH}$ stretch corresponds to the symmetric stretching coordinate for $\mathrm{H}_{2} \mathrm{O}$, viz., $v_{\mathrm{s}}$ ). The relative amplitudes of motion of the atoms in the molecule or group are readily determined by applying the condition of conservation of momentum to the vibration (this may not be strictly true for the group modes, but it appears not to be a bad approximation). The validity of the rule given by equation (44) can be 
seen in the excellent agreement between observed and predicted isotopic frequency ratios for the zero-order frequencies of $\nu_{1}, \nu_{2}$, and $\nu_{3}$ of $\mathrm{H}_{2} \mathrm{O}$ and $\mathrm{NH}_{3}$. In all of these cases the symmetry coordinate is a good approximation to the actual normal coordinate (which is quite well known for these molecules). In the case of $\nu_{4}$ of $\mathrm{NH}_{3}$ the simple symmetry coordinate itself does not approximate the true normal coordinate, and the slight discrepancy between observed and calculated ratios reflects this. Choice of the true normal coordinate in this case again results in excellent agreement. It will be noted that the ratios of the fundamental frequencies do not give as good agreement as those of the zero-order frequencies. This is to be expected, since the former include anharmonicity terms, which can often be large. Despite this, even the fundamental frequencies

Table 4. Application of Approximate Isotope Rule [Equation (44)] to Several Molecules and Groups

\begin{tabular}{|c|c|c|c|c|c|c|}
\hline \multirow{3}{*}{$\begin{array}{l}\begin{array}{c}\text { Molecule } \\
\text { or group }\end{array} \\
\begin{array}{l}\mathrm{H}_{2} \mathrm{O} \\
\mathrm{D}_{2} \mathrm{O}\end{array}\end{array}$} & \multirow[t]{2}{*}{ Mode } & \multirow{2}{*}{$\left(\frac{\nu}{v^{i}}\right)_{c a l c}$} & \multicolumn{4}{|c|}{$\left(\frac{y^{i}}{v^{i}}\right)_{o b s}$} \\
\hline & & & \multicolumn{2}{|c|}{ Fundamentals } & \multicolumn{2}{|c|}{ Zero-order freq. } \\
\hline & $\begin{array}{l}\nu_{1}: \text { sym. stretch } \\
\nu_{2}: \text { sym. bend } \\
\nu_{3}: \text { asym. stretch }\end{array}$ & $\begin{array}{l}1.384 \\
1.365 \\
1.365\end{array}$ & $\begin{array}{l}1.369^{1} \\
1.353 \\
1.348\end{array}$ & $\begin{array}{l}-1.1 \% \\
-0.9 \\
-1.2\end{array}$ & $\begin{array}{l}1.387^{1} \\
1.368 \\
1.363\end{array}$ & $\begin{array}{l}+0.2 \% \\
+0.2 \\
-0.2\end{array}$ \\
\hline $\begin{array}{l}\mathrm{NH}_{3} \\
\mathrm{ND}_{3}\end{array}$ & $\begin{array}{l}v_{1}: \text { sym. stretch } \\
\nu_{2}: \text { sym. bend } \\
\nu_{3}: \text { asym. stretch } \\
\nu_{4}: \text { asym. bend }\end{array}$ & $\begin{array}{l}1.395 \\
1.317 \\
1.359 \\
1.403\end{array}$ & $\begin{array}{l}1.3791 \\
1.270 \\
1.350 \\
1.367\end{array}$ & $\begin{array}{l}-1.1 \\
-3.6 \\
-0.7 \\
-2.6\end{array}$ & $\begin{array}{l}1.400^{1} \\
1.313 \\
1.357 \\
1.380\end{array}$ & $\begin{array}{r}+0.4 \\
-0.3 \\
-0.1 \\
-1.6\end{array}$ \\
\hline & $\begin{array}{l}\text { sym. stretch } \\
\text { asym. stretch } \\
\text { bend } \\
\text { rock } \\
\text { wag } \\
\text { twist }\end{array}$ & $\begin{array}{l}1.379 \\
1.349 \\
1.349 \\
1.379 \\
1.323 \\
1.414\end{array}$ & $\begin{array}{l}1.372^{2} \\
1.342 \\
1.341 \\
1.384\end{array}$ & $\begin{array}{r}-0.5 \\
-0.5 \\
-0.6 \\
+0.4\end{array}$ & & \\
\hline & $\begin{array}{l}\text { sym. stretch } \\
\text { asym. stretch } \\
\text { sym. bend } \\
\text { asym. bend } \\
\quad \text { rock }\end{array}$ & $\begin{array}{l}1.398 \\
1.349 \\
1.300 \\
1.403 \\
1.292\end{array}$ & $\begin{array}{l}1.372^{3} \\
1.330 \\
1.318 \\
1.375 \\
1.310\end{array}$ & $\begin{array}{l}-1.9 \\
-1.4 \\
+1.4 \\
-2.0 \\
+1.4\end{array}$ & $\begin{array}{l}1.388^{3} \\
1.350 \\
1.325 \\
1.391 \\
1.320\end{array}$ & $\begin{array}{l}-0.7 \\
+0.1 \\
+1.9 \\
+0.9 \\
+2.2\end{array}$ \\
\hline & $\begin{array}{ll}\mathrm{CH} \text { stretch: } & m_{x}=15 \\
& m_{x}=35.5 \\
\mathrm{CH} \text { bend: } & m_{x}=80 \\
& m_{x}=15 \\
& m_{x}=35.5 \\
& m_{x}=80\end{array}$ & $\begin{array}{l}1.320 \\
1.340 \\
1.355 \\
1.318 \\
1.322 \\
1.328\end{array}$ & $\begin{array}{l}1.340^{4} \\
1.340^{5} \\
\\
1.339^{4} \\
1.362^{5}\end{array}$ & $\begin{array}{r}0.0 \\
-1.1 \\
+1.3 \\
+2.6\end{array}$ & $\begin{array}{l}1.334^{4} \\
1.344^{5} \\
1.322^{4} \\
1.358^{5}\end{array}$ & $\begin{array}{r}-0.4 \\
-0.8 \\
0.0 \\
+2.3\end{array}$ \\
\hline $\begin{array}{l}1 \mathrm{DE} \\
2 \mathrm{Kr} \\
3 \mathrm{KI} \\
3 \mathrm{ZIF} \\
\mathrm{MH}_{\mathrm{H}}\end{array}$ & $\begin{array}{l}\text { SON (49) } \\
\text { MIANG, and SUTHE } \\
\text { MILLS, and CRAWFOR } \\
\text { W, CLEVELAND, and } \\
\text { ER, ROSSER, and ClE }\end{array}$ & $\begin{array}{l}\text { D) } \\
\text { STER } \\
\text { ND? }\end{array}$ & $\begin{array}{l}\text { 3) } \mathrm{CD} \\
\text { (CH }\end{array}$ & CD & & \\
\hline
\end{tabular}


give moderately good agreement with the predicted ratios: the average deviation has a magnitude of $1.2 \%$. In addition, the observed ratios for the fundamental frequencies order themselves exactly as do the predicted ratios. It is probable that if this isotope rule has utility, it will not be so much in the reproduction of the quoted magnitude of the isotopic frequency ratio for a given mode as in providing, through examination of such ratios for all bands involved, another cogent standard against which to measure a set of assignments. Some modes, such as the $\mathrm{CH}$ bend, seem to depend strongly on the local environment, so it may not be possible to use the quoted ratios for this case as confidently as in the other cases. The proper value for $\nu_{4}$ of $\mathrm{NH}_{3}$ (and $\mathrm{CD}_{3}$ ) is closer to 1.38 than to the 1.403 quoted in the table. Despite these limitations, the approximate isotopic frequency rule represented by equation (44) should significantly expand the utility of the technique of isotopic substitution in facilitating the assignment of hydrogen modes in the spectra of polymers.

\section{Polarized Radiation Studies}

We noted earlier (Section I. 1.) that the intensity of an absorption band is proportional to the square of the changing dipole moment in the molecule (i. e., transition moment) during the corresponding normal vibration. The intensity also depends upon the direction that the electric vector in the incident radiation makes with the transition moment. In particular, the intensity is proportional to the square of the scalar product of the transition moment and electric field vectors. This implies, for example, that if the electric field vector is perpendicular to the transition moment vector no absorption will occur. This fundamental relationship is the basis for the utilization of polarized infrared radiation as a powerful tool in the study of the spectra and structure of oriented polymers. We consider below some aspects of this technique.

In cases where the polymer chain (or crystal structure) exhibits symmetry, a qualitative knowledge of the polarization properties of an absorption band serves to associate the band with certain of the symmetry species, and therefore with certain of the normal modes of the molecule. Usually this does not uniquely define the mode, since several modes may belong to the same species, but in conjunction with other data it can help to narrow down the possibilities. In other cases it might unambiguously specify the normal mode. For example, as can be seen from Table 3 and Fig. 3 , the $B_{3 i} \mathrm{CH}_{2}$ wagging mode of a single planar zig-zag polyethylene chain is the only mode whose transition moment is parallel to the chain axis, and thus the identification of such a $\pi$ band in the spectrum which is associated with the planar zig-zag chain configuration would serve to assign this mode. Even in the case of the two $\pi$ 
bands which are predicted for crystalline polyethylene [KRIMM, LIANG, and SutHERLAND (102)], the polarization data serve to pick these $B_{3 u}$ modes out of the spectrum; other considerations would then make it possible to distinguish between them. In this particular case, $\pi$ bands could be detected if the sample were stretched (uniaxial orientation) or rolled (double orientation). Also the presence of disordered crystallites would not affect the result: the magnitude of the dichroic ratio would be altered by the presence of randomly oriented transition moments, but not the qualitative $\pi$ characteristic of the $B_{3 u}$ bands. (We are assuming that experimental errors associated with the measurement of dichroic ratios, such as those discussed in Section II. 2., are corrected for.) The same is true of $\sigma$ bands, except in the special case of a doubly oriented specimen in which the transition moment is parallel to the incident radiation: in this case the band would exhibit essentially no dichroism.

Another way in which polarized infrared studies can assist in the assignment of bands occurs when we are dealing with normal modes whose transition moments are mutually perpendicular. This characteristic would be true, for example, of the simple symmetric stretching, asymmetric stretching, and wagging modes of the $\mathrm{CH}_{2}$ group. For a sample with uniaxial orientation, the dichroic ratio for a mode whose transition moment makes an angle of $\theta$ with the fiber axis is given by [FRASER (58)]

$$
R_{0}=2 \cot ^{2} \theta
$$

Thus, a band will show $\pi, \sigma$, or no dichroism $(u)$ depending in whether $\theta$ is less than, greater than or equal to $54^{\circ} 44^{\prime}$. Although for three modes there are 10 possible combinations of $\pi, \sigma$, and $u$, it can be shown [LIANG and KRIMM (113)] that if the transition moments are mutually perpendicular then only four combinations are permitted: $\sigma \sigma \pi, \pi \pi \sigma, \pi \sigma u$, and $u u u$. Thus, for example, if the dichroism of the two $\mathrm{CH}_{2}$ stretching modes were known, that of the wagging mode would be determined. This knowledge could be of importance in assigning the latter since the band associated with it might occur in a region of complex absorption, so that a group frequency criterion alone would not be adequate.

In the above instances the qualitative polarization characteristics of a band served to provide information of importance in making assignments. If we make use of the quantitative aspects of the dichroic ratios of absorption bands then we can in addition obtain structural information about the polymer. In particular, it becomes possible to determine in many cases the direction which transition moments make with the fiber axis, which is often significant in establishing structural parameters. It must be noted that transition moment directions do not by themselves always serve to specify the orientation of chemical groups. In cases where 
the group has symmetry, such as $\mathrm{CH}_{2}$, it is to be expected (and experimental data bear this out) that the transition moments of the normal modes will be along or perpendicular to the symmetry element, in this case a two-fold rotation axis. However, where no such symmetry exists the transition moment direction for a normal mode is not necessarily determined by simple considerations of the nature of the vibration, but in fact usually requires detailed knowledge of the nature of the normal mode and of the importance of such factors as induced dipoles [HALFORD (73)] and orbital following [Coulson (41)]. A good example of such a situation is the problem of the transition moment directions of the normal modes of the amide group. Both experimental studies of single crystals containing this group [BAMFoRD, EllrotT, and HANBY (8), p. 184] and theoretical calculations of its normal modes [Mryazawa, Shimanouchi, and Mizushima (136)] indicate, for example, that the transition moment direction for the so-called $\mathrm{CO}$ stretching vibration makes an angle of about $20^{\circ}$ with the $\mathrm{CO}$ bond. It is therefore often necessary to exercise caution in the correlation of transition moment directions with bond directions, yet knowledge of the former is usually of significant help in answering certain structural questions.

In considering quantitative aspects of polarization studies we will restrict ourselves to the case of the uniaxially oriented polymer. The primary question is: given information on the dichroic ratio of absorption bands, is it possible to deduce the orientation of the corresponding transition moments with respect to the polymer chain axis? This is related to a second question: can we, from the infrared dichroism, deduce the distribution of chain (or crystallite) orientations? The latter question is of importance since, when a specimen is stretched, the chains do not all become perfectly oriented in the stretching direction but assume a distribution of orientations about this direction. If the form of this distribution is known, then it is possible to relate the dichroic ratio to the transition moment orientation [BAMFORD, ElLIOTT, and HANBY (8), p. 181; Fraser $(60)]$. However, this distribution is usually not known in any detail. The inverse process of deducing the form of the distribution from the dichroic ratio measurements is in general not possible. This is because several different distributions of the molecular chains may nevertheless give rise to the same distribution of dipoles, and therefore to the same dichroic ratio. Some general conclusions about the nature of the distribution can often be obtained from the results of tilting an oriented specimen at various angles to the polarized beam [LIANG and KRIMM (112)], but this does not provide adequate information. Another difficulty arises from the presence of randomly oriented material, which will alter the dichroic ratio from that calculated by equation (45). In some cases, however, it is possible to obtain information on the orientation of 
transition moments even though the chain distribution and the amount of randomly oriented material are not known.

It has been shown (BEER (12)] that under certain circumstances it is permissible to consider a polymer specimen as made up of a fraction, $f$, of fully oriented chains (i. e., with molecular axis exactly parallel with the fiber axis) and the remainder, $1-f$, of randomly oriented chains. The necessary condition is satisfied by axially oriented polymers, regardless of the specific nature of the distribution of chains. A convenient relationship between $f$, the observed dichroic ratio $R$, and the dichroic ratio of perfectly oriented chains $R_{0}=\varepsilon_{\pi} / \varepsilon_{\sigma}$ is given as follows (FRASER (59)]. In an axially oriented specimen the extinction coefficient in a given direction for the unoriented portion is simply related to $\varepsilon_{\pi}$ and $\varepsilon_{\sigma}$ for the oriented portion. Since the total extinction coefficient for the latter is given by $\varepsilon_{\pi}+2 \varepsilon_{\sigma}$, the extinction coefficient for the unoriented portion in a given direction is

$$
\varepsilon=\frac{1}{3}\left(\varepsilon_{\pi}+2 \varepsilon_{0}\right)
$$

Since

$$
R=\frac{f \varepsilon_{\pi}+(1-f) \varepsilon}{f \varepsilon_{\sigma}+(1-f) \varepsilon}
$$

we find, by substituting $R_{0}=\varepsilon_{n} / \varepsilon_{\sigma}$ and equation (46) into equation (47), that

$$
R=\frac{1+\frac{1}{3}\left(R_{0}-1\right)(1+2 f)}{1+\frac{1}{3}\left(R_{0}-1\right)(1-f)} .
$$

Graphs have been given [BAmFord, ElitotT, and Hanby (8)] which relate $R$ and $f$ for different values of $R_{0}$. This relation does not permit the determination of $R_{0}$ from $R$ (since $f$ is usually not known a priori), but it has been shown [FRASER (67)] that from the solution of equation (48) for $f$, viz.,

$$
f=\frac{(R-1)\left(\mathrm{R}_{0}+2\right)}{\left(R_{0}-1\right)(R+2)}
$$

it is often possible to place significant limits on $R_{0}$, and therefore on $\theta$. For certain configurations of transition moments it is possible to obtain $\theta$ uniquely [KRIMM (98)]. This occurs for certain cases of two mutually perpendicular transition moments, a situation which frequently occurs in polymers (e. g., the symmetric and asymmetric $\mathrm{CH}_{2}$ stretching modes). If two transition moments, $M_{1}$ and $M_{2}$, are perpendicular to each other, and $M_{2}$ makes an angle $\omega$ with the plane defined by $M_{1}$ and the molecular chain axis, then the angle, $\theta_{2}$, between $M_{2}$ and the chain axis is given by $\cos \theta_{2}=\cos \omega \sin \theta_{1}$, where $\theta_{1}$ is the angle between $M_{1}$ and the chain axis. If $R_{01}$ is given by equation (45), then

$$
R_{02}=\frac{4 \cos ^{2} \omega}{R_{01}+2 \sin ^{2} \omega} .
$$


By substituting in equation (49), and assuming the same value of $f$ for both bands, we find that

where

$$
\cot ^{2} \theta_{1}=\frac{A-B\left(1-3 \cos ^{2} \omega\right)}{2 A+B}
$$

$$
\begin{aligned}
& A=\left(R_{1}+2\right)\left(R_{2}-1\right) \\
& B=\left(R_{2}+2\right)\left(R_{1}-1\right) .
\end{aligned}
$$

Since $A$ and $B$ are experimentally determinable, $\theta_{1}$ can be obtained readily if $\omega$ is known (except for the case $\omega=\pi / 4$ ). In general this may not be the case, but for many polymers $\omega=0$ or $\omega=\pi / 2$ is true, i. e., $M_{2}$ is either in the plane of $M_{1}$ and the chain axis, or it is perpendicular to this plane. In such cases

and

$$
\cot ^{2} \theta_{1}=\frac{A+2 B}{2 A+B}, \quad \text { for } \omega=0
$$

$$
\cot ^{2} \theta_{1}=\frac{A-B}{2 A+B}, \quad \text { for } \omega=\frac{\pi}{2}
$$

In such instances no knowledge of the orientation distribution or of $f$ is required to compute $\theta_{1}$. Once $\theta_{1}$ is obtained, $f$ can be calculated from equation (49), and using this $f$ the $\theta$ values for other moments can be obtained directly from the observed dichroic ratios. These relations demonstrate the potentialities of polarized radiation studies in providing structural information on high polymers.

It is important to note that in the preceding discussion we have assumed that all of the transition moments along the polymer chain associated with a given normal mode of a repeat unit are independent of each other. That is, we have assumed an "oriented gas" of transition moments along the chain, analogous to the assumption often made in the study of the spectra of molecular crystals [Pimentel, MCCleldaN, Person, and Schnepp (173)]. This, of course, does not coincide with the factor group analysis (Section III. A. 1.) of the modes of a linear chain, which stipulates infrared and Raman activity only for those modes in which transition moments in all unit cells are in phase. The factor group analysis, which is certainly more valid than the "oriented gas" approach, nevertheless leads to the same result as equation (45), and therefore those following, if we can assume that the coupling between unit cells is weak [HIGGS (77)]. This implies that the motions of the atoms in a unit cell during a normal mode of the chain are essentially the same as they would be for an isolated unit cell. The various chain modes then differ only in the phase difference between the motions in neighbouring unit cells. A consequence of the weak interaction hypothesis is that the normal 
frequeuncies of all such modes are essentially independent of the phase difference, i. e., the $\pi$ and $\sigma$ chain modes occur at the same frequency, and as a result the dichroic ratio for a given kind of unit cell mode is also found to be given by equation (45). In many cases the assumption of weak coupling is not valid, as is evidenced by the lack of coincidence of the frequencies of the $\pi$ and $\sigma$ components of a band, and in some cases by the marked splitting in frequency of such components. This implies that the coupling is strong, viz., that motions in one unit cell are significantly influenced by similar motions in neighboring unit cells. Quantitative predictions of dichroism become quite complex in such cases because the effect of mutual induction can be to cause the transition moment for the entire chain to be not simply related to the sum of the moments of isolated unit cells. While this effect has been recognized in spectra of molecular crystals [HALFORD (73)], it has as yet not been conclusively demonstrated in polymer spectra. There is every reason to believe that it can occur, however, so that it must be kept in mind whenever a particular case is analyzed. It must also be remembered that interaction between chains in the crystalline regions of polymers can lead to additional splitting of frequencies. This will be particularly true of $\sigma$ bands, and must also be considered when quantitative measurements of dichroism are undertaken.

\section{Specimen Variation}

In analyzing the spectrum of a polymer we do not, of course, limit ourselves to the study of a single specimen. We have already noted the significant information which can be obtained by studying oriented samples as well as deuterated polymers, when possible. In this section we will consider briefly how the study of other kinds of specimens adds to our knowledge of the origin of bands.

Knowledge of characteristic group frequencies in large molecules, their location, intensity, variability, and so on, is of course enormously enhanced by detailed studies on small model compounds. Studies of this kind have been going on for years, and the resulting correlations are well catalogued [BELlAmY (13); JonEs and SANDORFy (82)]. The regularities which are observed in the spectra of small molecules can be important guides to the understanding of the complex spectra of high polymers, even if these regularities are only empirically established. The great advantage of studying small model compounds is that often the spectra of these have been analyzed in detail, so that inferences with respect to larger molecules can often be made from a secure base. Thus, detailed studies of the chlorine frequencies in the rotational isomers of 1,2 dichloroethane [Mrzushima (137)], and extension of this concept to, as well as experimental studies on, more complex chlorine-containing compounds 
[Mizushima, Shimanouchi, Nakamura, Hayashi, and Tsuchiya (139)], have furnished a significant basis for our understanding of the types of isomeric chain configurations which can exist in chlorine-containing high polymers. Studies of this type are dictated by the particular polymer under investigation. Sometimes the necessary information will be found in the literature; in other cases a careful study of such model compounds may have to be initiated in order to clarify certain aspects of the polymer spectrum.

The natural extension of the above idea to the particular case of spectral assignments in polymers has, however, not yet been made with full force. This would involve the study of small molecules which contain not just groups found in the polymer, but the basic repeating unit. That is, we should study molecules which consist of a few (or more) repeat units of the polymer in order that we may begin to understand the characteristic spectrum of the repeat unit as well as the spectral modifications which result from translation symmetry. Undoubtedly the best example of the use of this technique is the study of the spectra of n-paraffins as a means of achieving a more detailed understanding of the spectrum of polyethylene. Yet beyond this example there is surprisingly little work on low molecular weight analogues to high polymers. It should be pointed out that one of the major values of studying such small molecules is the feasibility of doing polarized infrared work on single crystals, which can often be obtained. The importance of such studies is that the dichroic behavior of the bands can be studied in greater detail since the crystal is usually orientable at will in the beam. This is usually not the case for the crystallites of an oriented polymer: for example, in a uniaxially oriented specimen only the direction of one axis of the crystallite is defined, the other two being distributed at random about this direction. Since, as we have seen, polarization data on the bands are of great importance in making assignments, any technique which provides such data is of major significance.

The study of analogue compounds which can be crystallized is also of importance in connection with another problem in spectral analysis. The spectrum of a typical polymer is usually a superposition of contributions from crystalline and amorphous regions. As must be clear by now, these contributions must be separated if we are to achieve a complete analysis. Crystalline analogue compounds provide a means for identifying the crystalline contribution to the polymer spectrum most simply. In their absence it is still possible, of course, to separate the contributions of the two phases. This may be done a) by preparing different samples of the polymer under polymerization conditions which give rise to varying degrees of crystallinity (as determined, say, by x-ray diffraction), and observing which bands change intensity and in which direction; and b) by 
preparing a completely amorphous specimen, either by melting the polymer or putting it in solution, and again making note of the intensity variation of the bands. (It might be noted that there seems to be evidence [KING, HAINER, and MCMAHON (89)] of an increase in crystallinity on lowering the temperature to $4^{\circ} \mathrm{K}$., but no systematic studies of this kind have been undertaken.) The analogue compounds can in addition, however, provide the means for a more detailed study of interactions which occur between molecules in the crystalline phase, an important factor in understanding the spectrum of a polymer. In this connection a technique which has been used in the case of n-paraffins [KRIMM, LIANG, and SutherLAND (702)] might well be applied to polymers in general. A means of detecting the presence of inter-chain interactions is to study the spectra of mixtures of normal and deuterated polymers. When, as in this case, a neighboring chain has different frequencies for comparable normal modes, any band splitting due to inter-chain interactions will disappear since the interaction between different frequencies is very much smaller. Such change in band contour thus qualitatively identifies the existence of an interaction.

In particular instances special kinds of experiments will be called for, but it should be clear that the study of varied specimens and analogous compounds is an important means by which we can obtain information which is directly relevant to the assignment of absorption bands to normal modes of the molecule.

\section{Spectra of High Polymers}

In part III the theoretical and experimental methods required for the detailed analysis of high polymer spectra were outlined. In this part we will discuss the spectra of some polymers for which such analyses have been undertaken. In many cases the above approach has only been partially applied, and this is reflected in the uncertainty which still exists about some of the assignments. Nevertheless, the progress which has been made in recent years by the application of many of the above techniques has resulted in a significant increase in our understanding of polymer spectra, and thereby various aspects of polymer structure. It will be the aim of the following sections to outline for various polymers our present understanding of their spectrum and its contribution to further knowledge of the structure and potential function of the polymer. Problems which remain to be answered with respect to obtaining a more secure set of assignments will be indicated. In most cases other aspects of the infrared spectrum will not be discussed. Some of the latter have been considered in a recent review article [SCHNELL (793)], [see also ELliott $(51 a)]$. 


\section{A. Polyethylene}

Polyethylene has been studied spectroscopically in greater detail than any other polymer. This is primarily a result of its (supposedly) simple structure and the hope that its simple spectrum could be understood in detail. Yet as simple as this structure and spectrum are, a satisfactory analysis had not been made until relatively recently, and even then significant problems of interpretation still remained. The main reason for this is that this polymer in fact generally contains structures other than the simple planar zig-zag implied by $-\left(\mathrm{CH}_{2} \mathrm{CH}_{2}\right)_{\bar{n}}$ : there are not only impurities of various kinds that differ chemically from the above, but the polymer always contains some amorphous material. In the latter portion of the material the chain no longer assumes an extended planar zig-zag conformation, and as we have noted earlier, such rotationally isomeric forms of a molecule usually have different spectra. Furthermore, the molecule has a center of symmetry, which as we have seen implies that some modes will be infrared inactive but Raman active, so that until Raman spectra became available recently it was difficult to be certain of the interpretation of some aspects of the spectrum. As a result of this work, and of detailed studies on the spectra of n-paraffins, it now seems possible to present a quite detailed assignment of bands in the vibrational spectrum of polyethylene.

A polarized infrared spectrum of a stretched sample of polyethylene is shown in Fig. 4 [Krimm, Liang, and SutherLand (102)]. This specimen was of moderate crystallinity and had a fairly

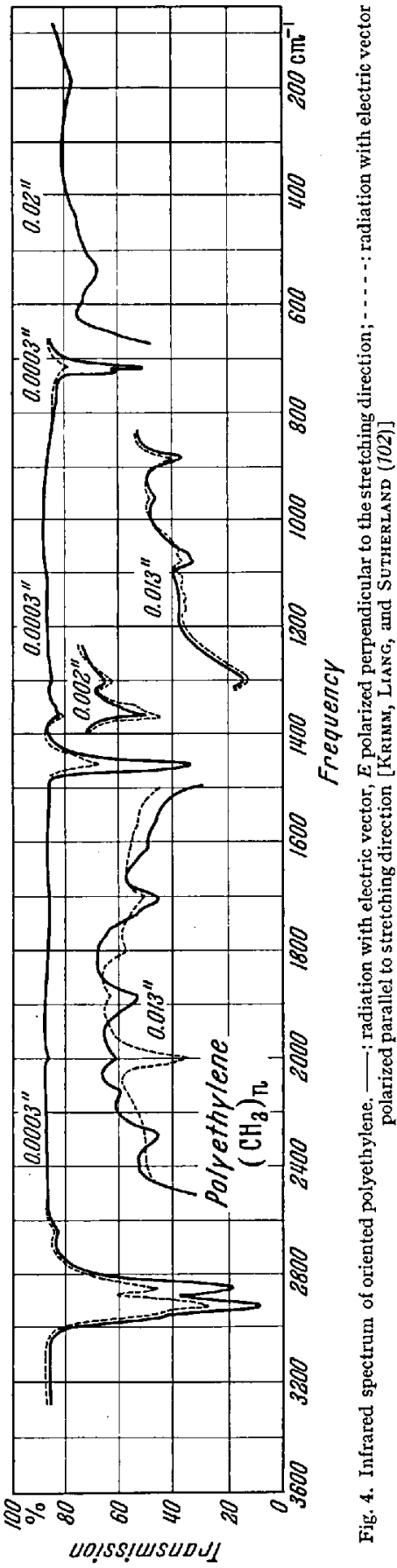


high $\mathrm{CH}_{3}$ group content (as measured by the intensity of the $1375 \mathrm{~cm}^{-1}$ band). Spectra of high crystallinity, low $\mathrm{CH}_{3}$ group content polyethylenes differ from this one primarily in the absence of the $1375 \mathrm{~cm}^{-1}$ band and in slight differences in relative intensities of some of the other bands [ToBIN and CARRANo (232); NIEL.SEN and WoOLLETT, $(160)]$. The band positions are listed in Table 5, together with their relative intensity, polarization with respect to the stretching direction of the specimen, and origin in the crystalline or amorphous regions. The frequencies of the interaction-split components are taken

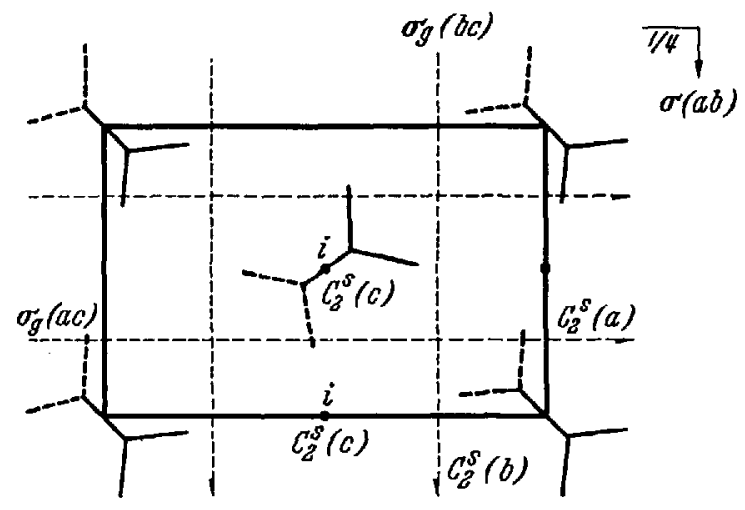

Fig. 5. Cross-scction perpendicular to the chain axis of unit cell of polyethylene, and symmetry elements. [KRIMM, Ltang, and SUTKERland (102)]

from studies of n-paraffin single crystals [KRIMM, LIANG, and SUTHERLAND (102)]. Bands in the overtone region are included [NIELSEN and Woorlett (160); GLATt and ElLIs (65)], as well as their polarization [NIELSEN (159)]. Lines observed in the Raman spectrum of polyethylene [Nielsen and Woollett (160)] are also shown in table 5. The assignments listed in the table will be discussed below.

The structure of the crystalline regions of polyethylene is well known [Bunn (26)]. The planar zig-zag chains crystallize in an orthorhombic unit cell of dimensions $a=7.40 \mathrm{~A}, b=4.93 \mathrm{~A}$, and $c=2.534 \mathrm{~A}$ (chain axis). There is evidence [WALTER and REDING (236)] that, depending on the degree of branching in the specimen, the former two spacings can vary between 7.36 and $7.68 \mathrm{~A}$ and 4.93 and $5.00 \mathrm{~A}$ respectively. The unit cell, which contains two chains, is shown in cross-section in Fig. 5 together with its associated symmetry elements. By the methods outlined in section III. A. 1. it is possible to obtain the character table describing the vibrational modes of such a crystal structure [ToBIN (230); KRIMM, Liang, and Sutherland (102)]. This is shown in Table 6. A set of symmetry modes for this structure is shown in Fig. 6. 
Table 5. Vibrational Spectrum of Polyethylene

\begin{tabular}{|c|c|c|c|c|c|}
\hline \multicolumn{2}{|c|}{$\begin{array}{c}\text { Frequency, } \\
\mathrm{cm}^{-1}\end{array}$} & \multicolumn{3}{|c|}{ Description $^{\mathbf{1}}$} & \multirow{2}{*}{ Assignment" } \\
\hline$I R$ & $\boldsymbol{R}$ & R.I. & Pol'n & Phase & \\
\hline$\sim 200$ & & $\mathrm{vw}$ & & & \\
\hline$\sim 430$ & & vVw & & & \\
\hline $\begin{array}{c}540-560 \\
573\end{array}$ & & $\mathrm{w}$ & & & \\
\hline $\begin{array}{r}573 \\
\sim 600\end{array}$ & & $\begin{array}{l}w \\
w\end{array}$ & $\pi$ & & $\mathrm{RCH}=\mathrm{CH}_{2}$ \\
\hline 720 & & vs & $\sigma_{b}$ & C, $\mathrm{A}$ & $\gamma_{r}\left(\mathrm{CH}_{2}\right)\left(\mathrm{B}_{2 u}\right) ; \gamma_{r}\left(\mathrm{CH}_{2}\right)-\mathrm{t}$ \\
\hline 731 & & vs & $\sigma_{a}$ & C & $\gamma_{r}\left(\mathrm{CH}_{2}\right)\left(\mathrm{B}_{14}\right)$ \\
\hline 888 & & $\mathrm{vw}$ & $\sigma$ & & $\mathrm{RR}^{\prime} \mathrm{C}=\mathrm{CH}_{2}$ \\
\hline $\begin{array}{l}890 \\
908\end{array}$ & & $\begin{array}{c}\mathrm{vvw} \\
\mathrm{m}\end{array}$ & $\begin{array}{l}\pi \\
\sigma\end{array}$ & $A, C$ & $\begin{array}{l}\gamma_{r}\left(\mathrm{CH}_{3}\right) \\
\mathrm{RCH}=\mathrm{CH}_{2}\end{array}$ \\
\hline 964 & & vvw & $\sigma$ & A & $\left\{\begin{array}{l}\gamma_{r}\left(\mathrm{CH}_{2}\right)-\mathrm{g} \\
\text { trans RCH }=\mathrm{CHR}^{\prime}\end{array}\right.$ \\
\hline 990 & & $\mathrm{w}$ & $\sigma$ & A, C & $\mathrm{RCH}=\mathrm{CH}_{2}$ \\
\hline 1050 & 1061 & $\begin{array}{c}\mathrm{vw} \\
\mathrm{m}\end{array}$ & $\pi$ & $\mathrm{C}$ & $\gamma_{1}\left(\mathrm{CH}_{2}\right)\left(\mathrm{B}_{3 u}\right)$ \\
\hline 1065 & & $\mathrm{vw}$ & $\sigma$ & & $\boldsymbol{y}_{+}(0)$-amorphous $(?)$ \\
\hline 1078 & & $\mathrm{vw}$ & $\sigma$ & A & $v(\mathrm{C}-\mathrm{C})-\mathrm{t}, \mathrm{g}$ \\
\hline 1110 & & vvw & $\pi$ & & \\
\hline 1131 & 1131 & $\begin{array}{c}\mathrm{vvw} \\
\mathrm{m}\end{array}$ & $\pi$ & & $\begin{array}{l}v_{+}(\pi) \text {-amorphous }(?) \\
v_{+}(\pi)\left(B_{2 \sigma}\right)\end{array}$ \\
\hline 1150 & 1168 & & $\pi$ & & $\gamma_{r}\left(\mathrm{CH}_{2}\right)\left(\mathrm{A}_{\sigma}\right)$ \\
\hline $\begin{array}{l}1170 \\
1176 \\
1185\end{array}$ & & $\begin{array}{l}\text { vvw } \\
\text { vw } \\
\text { vvw }\end{array}$ & $\begin{array}{l}\sigma \\
\pi \\
\sigma\end{array}$ & $\mathrm{C}$ & $\begin{array}{l}\gamma_{r}\left(\mathrm{CH}_{2}\right)\left(\mathrm{A}_{o}\right) \text {-amorphous (?) } \\
\gamma_{u}\left(\mathrm{CH}_{2}\right)\left(\mathrm{B}_{3 u}\right)\end{array}$ \\
\hline 1303 & 1295 & $\mathrm{~s}$ & $\pi$ & A & $\gamma_{1}\left(\mathrm{CH}_{2}\right)\left(\mathrm{B}_{2 \sigma}\right)$ \\
\hline 1353 & & $\mathrm{~m}$ & $\pi$ & A & $\begin{array}{l}\gamma_{1}(\mathrm{CH} 2)_{2}-\mathrm{g} \\
\gamma_{\infty}(\mathrm{CH})_{2}-\mathrm{g}\end{array}$ \\
\hline 1369 & & $\mathrm{~m}$ & $\pi$ & $A, C(?)$ & $\left.\gamma_{w} \mathrm{CH}_{2}\right)-\mathrm{g}, \mathrm{t}(?)$ \\
\hline 1375 & & $\mathrm{w}-\mathrm{m}$ & $\pi(?)$ & A & $\delta_{2}\left(\mathrm{CH}_{3}\right)$ \\
\hline 1436 & 1415 & $\begin{array}{c}\mathrm{w} \\
\mathrm{vw}\end{array}$ & & & $\begin{array}{l}\gamma_{20}\left(\mathrm{CH}_{2}\right)\left(\mathrm{B}_{2 g}\right) \\
\delta\left\langle\mathrm{CH}_{2}\right)\left(\mathrm{A}_{g}\right) \text {-amorphous (?) }\end{array}$ \\
\hline 1457 & 1440 & $\underset{\text { vvw }}{\mathrm{m}}$ & & & $\begin{array}{l}\delta\left(\mathrm{CH}_{2}\right)\left(\mathrm{A}_{g}\right) \\
\delta_{a}\left(\mathrm{CH}_{3}\right)\end{array}$ \\
\hline 1463 & 1464 & $\begin{array}{l}\text { vs } \\
\mathrm{w}, \mathrm{b}\end{array}$ & $\sigma_{b}$ & C, $\mathrm{A}$ & $\begin{array}{l}\delta\left(\mathrm{CH}_{2}\right)\left(\mathrm{B}_{2 u}\right) ; \delta\left(\mathrm{CH}_{2}\right)-\mathrm{t}_{1} \mathrm{~g} \\
2 \times \gamma_{r}\left(\mathrm{~B}_{1 u}\right)=1462\left(\mathrm{~A}_{\sigma}\right)\end{array}$ \\
\hline $\begin{array}{l}1473 \\
1588\end{array}$ & & $\begin{array}{l}\text { vs } \\
\text { vvw }\end{array}$ & $\begin{array}{c}\sigma_{a} \\
\sigma\end{array}$ & $\begin{array}{l}\mathrm{C} \\
\mathrm{C}\end{array}$ & $\begin{array}{l}\delta\left(\mathrm{CH}_{2}\right)\left(\mathrm{B}_{1 u}\right) \\
1050+540=1590(?)\end{array}$ \\
\hline 1640 & & vw & $\pi$ & $A, C$ & $v(\mathrm{C}=\mathrm{C})$ \\
\hline 1710 & & vw & $\pi$ & & $1168+540=1708(?)$ \\
\hline $\begin{array}{l}1722 \\
1737\end{array}$ & & $\begin{array}{l}\mathrm{vw} \\
\mathrm{vvw}\end{array}$ & $\sigma$ & & $\begin{array}{l}v(\mathrm{C}=\mathrm{O}) \\
v(\mathrm{C}=\mathrm{O})\end{array}$ \\
\hline$\sim 1805$ & & vw & $\pi$ & A, C & $\left\{\begin{array}{l}v(1078)+\gamma_{r}(720)=1798 \\
2 \times 908=1816\end{array}\right.$ \\
\hline $\begin{array}{l}1894 \\
1960\end{array}$ & & $\underset{\mathrm{wvw}}{\mathrm{w}}$ & $\stackrel{\sigma}{\pi(?)}$ & $\mathrm{C}$ & $\begin{array}{l}\gamma_{r}\left(\mathrm{~A}_{\sigma}\right)+\gamma_{r}\left(\mathrm{~B}_{1 u}\right)=1899\left(\mathrm{~B}_{1 \mathrm{u}}\right) \\
2 \times 990=1980(?)\end{array}$ \\
\hline 2016 & & $\mathrm{w}$ & $\pi$ & C, $\mathrm{A}$ & $\left\{\begin{array}{l}\gamma_{t}\left(\mathrm{~B}_{2 g}\right)+\gamma_{r}\left(\mathrm{~B}_{1 u}\right)=2026\left(\mathrm{~B}_{3 u}\right) \\
\gamma_{t}(1303)+\gamma_{r}(720)=2023\end{array}\right.$ \\
\hline 2144 & & vw & $\sigma$ & $\mathrm{C}, \mathrm{A}$ & $\left\{\begin{array}{l}\delta\left(\mathrm{A}_{v}\right)+\gamma_{r}\left(\mathrm{~B}_{2 u}\right)=2160\left(\mathrm{~B}_{2 w}\right) \\
2 \times v(1078)=2156\end{array}\right.$ \\
\hline 2221 & & vw & $\pi$ & & $\gamma_{r}\left(A_{\rho}\right)+\gamma_{t}\left(B_{3 u}\right)=2218\left(B_{3 u}\right)$ \\
\hline 2242 & & vw & $\pi$ & $\mathrm{C}$ & $\gamma_{w}\left(\mathrm{~B}_{3 u}\right)+\nu_{+}(0)\left(\mathrm{A}_{g}\right)=2237\left(\mathrm{~B}_{3 u}\right)$ \\
\hline 2290 & & $\mathrm{vw}$ & $\sigma$ & $\mathrm{C}$ & $\gamma_{v}\left(\mathrm{~B}_{2 u}\right)+\nu_{+}(\pi)\left(\mathrm{B}_{2 q}\right)=2307\left(\mathrm{~B}_{1 u}\right)$ \\
\hline
\end{tabular}


Table 5. (Continuation)

\begin{tabular}{|c|c|c|c|c|c|}
\hline \multicolumn{2}{|c|}{$\begin{array}{l}\text { Frequency, } \\
\qquad \mathrm{cm}^{-1}\end{array}$} & \multicolumn{3}{|c|}{ Description ${ }^{2}$} & \multirow{2}{*}{ Assignment ${ }^{2}$} \\
\hline$I R$ & $R$ & R.I. & Pol'n & Phase & \\
\hline 2328 & & $\mathbf{w}$ & $\sigma$ & & $\left\{\begin{array}{l}\gamma_{w}(1369)+\gamma_{r}(964)=2333 \\
2 \times \gamma_{r}(1168)=2336\end{array}\right.$ \\
\hline 2345 & & $\mathrm{w}$ & $\sigma$ & $\mathrm{C}, \mathrm{A}$ & $\gamma_{t}\left(\mathrm{~B}_{2 g}\right)+\gamma_{l}\left(\mathrm{~B}_{3 u}\right)=2345\left(\mathrm{~B}_{1 u}\right)$ \\
\hline 2415 & & vw & $\sigma$ & $A, C$ & $\left\{\begin{array}{l}v(1078)+\gamma_{r r}(1353)=2431 \\
\gamma_{r}(964)+\delta(1463)=2427\end{array}\right.$ \\
\hline 2473 & & vvw & $\sigma$ & $\mathrm{C}, \mathrm{A}$ & $\left\{\begin{array}{l}\left\{\gamma_{t}\left(\mathrm{~B}_{2 g}\right)+\gamma_{v o}\left(\mathrm{~B}_{3 u}\right)=2471\left(\mathrm{~B}_{14}\right)\right. \\
\gamma_{r}(1168)+\gamma_{t}(1303)=2471 \\
v(1078)+\delta(1440)=2518\end{array}\right.$ \\
\hline 2525 & & $\mathrm{vw}$ & $\pi$ & & $\left\{\begin{array}{l}\gamma_{r}(1168)+\gamma_{w}(1353)=2521 \\
\gamma_{r}(1168)+\gamma_{w}(1369)=2537\end{array}\right.$ \\
\hline 2549 & & vvw & $\sigma$ & & $v(1078)+\delta(1463)=2541$ \\
\hline 2597 & & vvw & $\sigma(?)$ & & $\gamma_{w v}\left(B_{2 g}\right)+\gamma_{w s}\left(B_{3 u}\right)=2591\left(B_{1 u}\right)$ \\
\hline $\begin{array}{l}2619 \\
2635\end{array}$ & & vvw & $\pi$ & & $\delta\left(\mathrm{A}_{g}\right)+\gamma_{t o}\left(\mathrm{~B}_{3 u}\right)=2616\left(\mathrm{~B}_{3 u}\right)$ \\
\hline 2658 & & $\begin{array}{l}w \\
w\end{array}$ & $\begin{array}{l}\sigma \\
\sigma\end{array}$ & C & $\gamma_{r}\left(\mathrm{~A}_{\mathrm{g}}\right)+\delta\left(\mathrm{B}_{1 \mathrm{u}}\right)=2641\left(\mathrm{~B}_{1 \mathrm{u}}\right)$ \\
\hline 2677 & & vvw & $\pi(?)$ & $\mathrm{A}$ & $\begin{array}{l}\gamma_{w o}(1353)+\gamma_{t}(1303)=2656 \\
\gamma_{w}(1369)+\gamma_{1}(1303)=2672\end{array}$ \\
\hline 2713 & 2720 & $\begin{array}{l}\text { vvw } \\
\text { vvw, b }\end{array}$ & & & $\begin{array}{l}2 \times \gamma_{v o}(1353)=2706 \\
\gamma_{g}\left(\mathrm{~B}_{2 q}\right)+\delta\left(\mathrm{A}_{g}\right)=2735\left(\mathrm{~B}_{2 g}\right)\end{array}$ \\
\hline 2741 & 2848 & $\begin{array}{l}\text { vvw } \\
\text { vs, b }\end{array}$ & $\sigma$ & & 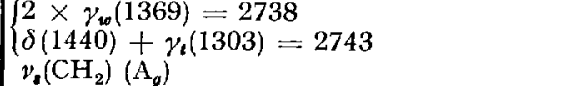 \\
\hline $\begin{array}{l}2850 \\
2857\end{array}$ & & vvs & $\sigma_{a}$ & A, C & $v_{d}\left(\mathrm{CH}_{2}\right)\left(\mathrm{B}_{\mathrm{It}}\right) ; v_{d}\left(\mathrm{CH}_{2}\right)-\mathrm{t}, \mathrm{g}$ \\
\hline 2874 & & $\begin{array}{l}\mathrm{vvs} \\
\mathrm{w}\end{array}$ & $\sigma_{b}$ & A & $\begin{array}{l}v_{a}\left(\mathrm{CH}_{2}\right)\left(\mathrm{B}_{2 u}\right) \\
v_{s}\left(\mathrm{CH}_{3}\right)\end{array}$ \\
\hline 2899 & $\begin{array}{l}2883 \\
2912\end{array}$ & $\begin{array}{l}\text { vvs } \\
\text { vvs } \\
\text { w }\end{array}$ & $\sigma_{b}$ & C & $\begin{array}{l}v_{a}\left(\mathrm{CH}_{2}\right)\left(\mathrm{A}_{g}\right) \\
v_{a}\left(\mathrm{CH}_{2}\right)\left(\mathrm{B}_{2 u}\right) \\
\delta\left(\mathrm{A}_{o}\right)+2 \times \gamma_{(}\left(\mathrm{B}_{1 u}\right)=2902\left(\mathrm{~A}_{\jmath}\right) \\
2 \times \delta\left(\mathrm{B}_{2 u}\right)=2926\left(\mathrm{~A}_{\sigma}\right)\end{array}$ \\
\hline 2960 & 2932 & $\begin{array}{l}\text { vvs } \\
\mathrm{w} \\
\mathrm{w}\end{array}$ & $\sigma_{a}$ & $A, C$ & $\begin{array}{l}v_{a}\left(\mathrm{CH}_{2}\right)\left(\mathrm{B}_{1 u}\right) ; v_{a}\left(\mathrm{CH}_{2}\right)-\mathrm{t}, \mathrm{g} \\
\delta\left(\mathrm{B}_{1 u}\right)+\delta\left(\mathrm{B}_{2 u}\right)=2936\left(\mathrm{~B}_{3 q}\right) \\
v_{a}\left(\mathrm{CH}_{3}\right)\end{array}$ \\
\hline $\begin{array}{l}3080 \\
3274\end{array}$ & & $\begin{array}{l}\text { vw } \\
\text { vvw }\end{array}$ & $\begin{array}{l}\pi \\
\pi\end{array}$ & & $\begin{array}{l}\gamma_{t}\left(\mathrm{~B}_{2 o}\right)+\nu_{+}(0)\left(\mathrm{A}_{g}\right)+\gamma_{r}\left(\mathrm{~B}_{1 u}\right)=3087\left(\mathrm{~B}_{3 u}\right) \\
2 \times v(\mathrm{C}=\mathrm{C})=3280\end{array}$ \\
\hline 3371 & & vrw & $\pi$ & $A, C$ & $v(\hat{\mathrm{OH}})$ \\
\hline 3433 & & vvw & $\sigma$ & & $\begin{array}{l}\gamma_{v o}\left(\mathrm{~B}_{2 \sigma}\right)+\gamma_{t}\left(\mathrm{~B}_{2 g}\right)+\gamma_{r}\left(\mathrm{~B}_{1 u, 2 u}\right) \\
=3436\left(\mathrm{~B}_{1 u, 2 u}\right)\end{array}$ \\
\hline 3648 & & $\mathrm{w}$ & $\sigma$ & $\mathrm{C}, \mathrm{A}$ & $\begin{array}{l}\left\{\begin{array}{l}v_{a}\left(\mathrm{~A}_{\sigma}\right)+\gamma_{r}\left(\mathrm{~B}_{1 u}\right)=3614\left(\mathrm{~B}_{1 u}\right) \\
v_{a}(2883)+\gamma_{r}(720)=3603\end{array}\right. \\
\left(2 \times \gamma_{t}\left(\mathrm{~A}_{\sigma}\right)+\gamma_{t}\left(\mathrm{~B}_{3 u}\right)=3640\left(\mathrm{~B}_{3 u}\right)\right.\end{array}$ \\
\hline$\sim 3700$ & & $\mathrm{w}$ & $\pi$ & & $\left\{\begin{array}{l}\delta\left(\mathrm{B}_{1 u s}\right)+\nu_{+}(\pi)\left(\mathrm{B}_{2 g}\right)+\nu_{+}(0)\left(\dot{\mathrm{A}}_{o}\right) \\
\quad=3665\left(\mathrm{~B}_{3 u}\right)\end{array}\right.$ \\
\hline$\sim 3700$ & & vvw & $\pi(?)$ & & $\begin{array}{l}\gamma_{w}\left(\mathrm{~B}_{2 g}\right)+\gamma_{w}\left(\mathrm{~B}_{3 u}\right)+v_{+}(\pi)\left(\mathrm{B}_{2 g}\right) \\
\quad=3722\left(\mathrm{~B}_{3 u}\right)\end{array}$ \\
\hline 3810 & & vvw & $\sigma$ & & $2 \times \gamma_{r}\left(\mathrm{~A}_{\vartheta}\right)+\delta\left(\mathrm{B}_{1 u}\right)=3809\left(\mathrm{~B}_{1 u}\right)$ \\
\hline 3876 & & vvw & $\sigma$ & & $\delta\left(\mathrm{B}_{2 u}\right)+\gamma_{s}\left(\mathrm{~B}_{2 g}\right)+\nu_{+}(\pi)\left(\mathrm{B}_{2 \sigma}\right)=3889\left(\mathrm{~B}_{2 u}\right)$ \\
\hline $\begin{array}{l}3900 \\
3982\end{array}$ & & $\begin{array}{l}\text { vvw } \\
\text { vyw }\end{array}$ & $\pi$ & & $v_{s}\left(\mathrm{~A}_{g}\right)+\gamma_{l}\left(\mathrm{~B}_{3 u}\right)=3898\left(\mathrm{~B}_{3 u}\right)$ \\
\hline 3989 & & vvw & $\pi$ & & $\begin{array}{l}v_{a}\left(\mathrm{~B}_{1 u}\right)+v_{+}(0)\left(\mathrm{A}_{g}\right)=3985\left(\mathrm{~B}_{1 u t}\right) \\
v_{g}\left(\mathrm{~B}_{1 u}\right)+v_{+}(\pi)\left(\mathrm{B}_{2 g}\right)=3981\left(\mathrm{~B}_{3 u}\right)\end{array}$ \\
\hline 4027 & & $\mathrm{vw}$ & $\sigma$ & & $v_{s}\left(\mathrm{~B}_{2 u}\right)+\gamma_{r}\left(\mathrm{~A}_{g}\right)=4025\left(\mathrm{~B}_{2 u}\right)$ \\
\hline 4055 & & vvw & $\pi$ & & $\left\{\begin{array}{l}v_{a}\left(\mathrm{~B}_{1 u}\right)+v_{+}(\pi)\left(\mathrm{B}_{2 a}\right)=4055\left(\mathrm{~B}_{3 u}\right) \\
v_{a}\left(\mathrm{~A}_{a}\right)+\gamma_{u}\left(\mathrm{~B}_{3 u}\right)=4059\left(\mathrm{~B}_{a u}\right)\end{array}\right.$ \\
\hline $\begin{array}{l}4106 \\
4185\end{array}$ & & $\begin{array}{l}w \\
w\end{array}$ & $\begin{array}{l}\sigma \\
\sigma\end{array}$ & & $\begin{array}{l}v_{a}\left(\mathrm{~B}_{1 u}\right)+\gamma_{r}\left(\mathrm{~A}_{g}\right)=4092\left(\mathrm{~B}_{1 u}\right) \\
v_{a}(2883)+\gamma_{b}(1303)=4186\end{array}$ \\
\hline
\end{tabular}


Table 5. (Continuation)

\begin{tabular}{|c|c|c|c|c|c|}
\hline \multicolumn{2}{|c|}{$\begin{array}{l}\text { Frequency, } \\
\mathrm{cm}^{-1}\end{array}$} & \multicolumn{3}{|c|}{ Description $^{1}$} & \multirow{2}{*}{ Assignment ${ }^{2}$} \\
\hline$I R$ & $R$ & R. I. & Pol'n & Phase & \\
\hline 4221 & & $\mathrm{w}$ & $\pi$ & & $\nu_{a}\left(\mathrm{~B}_{1 u}\right)+\gamma_{l}\left(\mathrm{~B}_{2 g}\right)=4219\left(\mathrm{~B}_{3 u}\right)$ \\
\hline 4255 & & $\mathrm{mw}$ & $\sigma$ & & $\begin{array}{c}v_{a}(2883)+\gamma_{v o}(1369)=4252 \\
\left(v_{s}\left(\mathrm{~A}_{o}\right)+\delta\left(\mathrm{B}_{1 u}\right)=4321\left(\mathrm{~B}_{1 u}\right)\right.\end{array}$ \\
\hline 4327 & & $\mathrm{~m}$ & $\sigma$ & & $\left\{\begin{array}{l}v_{a}\left(\mathrm{~B}_{2 u}\right)+\delta\left(\mathrm{A}_{a}\right)=4339\left(\mathrm{~B}_{2 u}\right) \\
v_{a}\left(\mathrm{~A}_{a}\right)+\delta\left(\mathrm{B}_{2 u}\right)=4346\left(\mathrm{~B}_{2 u}\right)\end{array}\right.$ \\
\hline 4629 & & vow & $\sigma$ & & 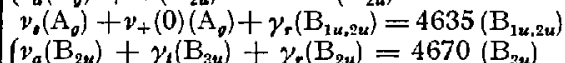 \\
\hline 4695 & & $\mathrm{vw}$ & $\pi$ & & $\left\{\begin{array}{l}v_{a}\left(\mathrm{~B}_{1 u}\right)+\gamma_{t}\left(\mathrm{~B}_{3 s}\right)+\gamma_{r}\left(\mathrm{~B}_{1 u}\right)=4705\left(\mathrm{~B}_{2 u}\right) \\
\nu_{s}\left(\mathrm{~A}_{a}\right)+v_{+}(\pi)\left(\mathrm{B}_{2 a}\right)+\gamma_{r}\left(\mathrm{~B}_{1 u}\right)=4710\left(\mathrm{~B}_{2,}\right)\end{array}\right.$ \\
\hline 4888 & & $\mathrm{~ms}$ & $\pi$ & & $\begin{array}{l}v_{s}, v_{a}\left(\mathrm{~A}_{g}\right)+\gamma_{b}\left(\mathrm{~B}_{2 g}\right)+\gamma_{s}\left(\mathrm{~B}_{u u}\right)=4892\left(\mathrm{~B}_{3 u}\right) \\
\left(v_{u}, v_{a}\left(\mathrm{~B}_{1 u, 2 u}\right)+2 \times \gamma_{l}\left(\mathrm{~B}_{3 u}\right)=4983\left(\mathrm{~B}_{1 u, 2 u}\right)\right.\end{array}$ \\
\hline 4987 & & w & $\sigma$ & & $\left\{\begin{array}{l}v_{s}, v_{a}\left(\mathrm{~B}_{1 u, 2 u}\right)+2 \times v_{+}(0)\left(\mathrm{A}_{a}\right) \\
=5004\left(\mathrm{~B}_{1 u, 2 u}\right)\end{array}\right.$ \\
\hline 5076 & & $\mathrm{vw}$ & $\pi$ & & $\begin{array}{l}v_{s}, v_{a}\left(\mathrm{~B}_{1 u}\right)+v_{+}(\pi)\left(\mathrm{B}_{2 q}\right)+v_{+}(0)\left(\mathrm{A}_{q}\right) \\
\quad=5079\left(\mathrm{~B}_{3 u}\right)\end{array}$ \\
\hline 5107 & & vw & $\sigma$ & & $\begin{array}{l}v_{z}, v_{a}\left(\mathrm{~B}_{2 u}\right)+\gamma_{r}\left(\mathrm{~A}_{0}\right)+v_{+}(0)\left(\mathrm{A}_{a}\right) \\
\quad=5107\left(\mathrm{~B}_{2 u}\right)\end{array}$ \\
\hline 5181 & & VW & $\pi$ & & $\begin{array}{l}v_{a r} \\
\quad=5186\left(\mathrm{~B}_{1 u}\right)+\gamma_{r}\left(\mathrm{~A}_{q}\right)+v_{+}(\pi)\left(\mathrm{B}_{2 q}\right)\end{array}$ \\
\hline 5397 & & $\mathrm{vwr}$ & $\pi$ & & $\left\{\begin{array}{l}v_{s}\left(\mathrm{~B}_{1 u}\right)+\delta\left(\mathrm{B}_{1 u}\right)+\gamma_{s}\left(\mathrm{~B}_{3 u}\right)=5373\left(\mathrm{~B}_{3 u}\right) \\
v_{a}\left(\mathrm{~B}_{2 u}\right)+\delta\left(\mathrm{B}_{2 u}\right)+\gamma_{t}\left(\mathrm{~B}_{3 u}\right)=5412\left(\mathrm{~B}_{3 u}\right)\end{array}\right.$ \\
\hline 5453 & & vow & $\pi$ & & $\left\{\begin{array}{l}\nu_{a}\left(\mathrm{~B}_{1 u}\right)+\delta\left(\mathrm{B}_{1 u}\right)+\gamma_{l}\left(\mathrm{~B}_{3 u}\right)=5447\left(\mathrm{~B}_{3 u}\right) \\
v_{g}\left(\mathrm{~A}_{q}\right)+\delta\left(\mathrm{A}_{q}\right)+\gamma_{w}\left(\mathrm{~B}_{3 u}\right)=5464\left(\mathrm{~B}_{3 u}\right)\end{array}\right.$ \\
\hline 5488 & & $\begin{array}{l}v w \\
w\end{array}$ & $\begin{array}{l}\sigma \\
\sigma\end{array}$ & & $\begin{array}{l}v_{s}\left(\mathrm{~A}_{g}\right)+\delta\left(\mathrm{B}_{1 u}\right)+\gamma_{s}\left(\mathrm{~A}_{g}\right)=5489\left(\mathrm{~B}_{1 u}\right) \\
v_{s}\left(\mathrm{~A}_{g}\right)+v_{s}\left(\mathrm{~B}_{1 u}\right)=5698\left(\mathrm{~B}_{1 u}\right)\end{array}$ \\
\hline 5770 & & $\mathrm{~m}$ & $\sigma$ & & $v_{\sigma}\left(\mathrm{A}_{g}\right)+v_{a}\left(\mathrm{~B}_{2 u}\right)=5782\left(\mathrm{~B}_{2 u}\right)$ \\
\hline
\end{tabular}

$1 \mathrm{vs}=$ very strong, $\mathrm{m}=$ medium, $\mathrm{w}=$ weak, $\mathrm{b}=$ broad, $\pi=$ parallel, $\sigma=$ perpendicular $\left[\sigma_{a(b)}=\right.$ polarization along $a(b)$ crystal axis $], \mathrm{A}=$ amorphous, $\mathrm{C}=$ crystalline.

$v=$ stretching, $\delta$ = bending, $\gamma_{w}=$ wagging, $\gamma_{t}=$ twisting, $\gamma_{r}=$ rocking Amorphous chain fundamentals and combinations do not carry species designation. $t=$ trans, $g=$ gauche configuration.

Table 6. Character Table and Selection Rules for Crystalline Polyethylene

\begin{tabular}{|c|c|c|c|c|c|c|c|c|c|c|c|c|c|c|c|}
\hline$D_{2 h}$ & $E$ & $C_{\mathbf{s}}^{\prime}(a)$ & $C_{\mathbf{s}}^{\boldsymbol{s}(b)}$ & $C_{2}^{\prime}(c)$ & $i$ & $\sigma_{\theta}(b c)$ & $\sigma_{g}(a c)$ & $\sigma(a b)$ & $n_{i}$ & $T$ & $T^{\prime}$ & $R^{\prime}$ & $n_{i}^{\prime}$ & $I R$ & $R$ \\
\hline$A_{p}$ & 1 & 1 & 1 & 1 & 1 & 1 & 1 & 1 & 6 & 0 & 2 & 1 & 3 & f & $\mathrm{P}$ \\
\hline$B_{1 g}$ & 1 & 1 & -1 & -1 & 1 & 1 & -1 & -1 & 3 & 0 & 1 & 2 & 0 & $f$ & $\mathrm{D}$ \\
\hline $\mathrm{B}_{2 \sigma}$ & 1 & -1 & 1 & -1 & 1 & $\ldots 1$ & 1 & -1 & 3 & 0 & $I$ & 2 & 0 & f & D \\
\hline $\mathrm{B}_{3 g}$ & 1 & -1 & -1 & 1 & 1 & -1 & -1 & 1 & 6 & 0 & 2 & 1 & 3 & $f$ & $\mathrm{D}$ \\
\hline$A_{u}$ & 1 & 1 & 1 & 1 & -1 & -1 & -1 & -1 & 3 & 0 & 1 & 2 & 0 & f & $f$ \\
\hline$B_{1 u}$ & 1 & 1 & -1 & -1 & -1 & -1 & 1 & 1 & 6 & $T_{a}$ & 1 & 1 & 3 & $\mathrm{a}$ & f \\
\hline $\mathrm{B}_{2 u}$ & 1 & -1 & 1 & -1 & -1 & 1 & -1 & 1 & 6 & $T_{b}$ & 1 & 1 & 3 & a & $f$ \\
\hline $\mathrm{B}_{3 u}$ & 1 & -1 & -1 & 1 & -1 & 1 & 1 & -1 & 3 & $\mathrm{~T}_{e}$ & 0 & 2 & 0 & $a$ & $f$ \\
\hline
\end{tabular}

If the symmetry analysis for the unit cell is compared with that for the single chain (section III. A. 1.), several interesting features of the vibrational spectrum are seen to emerge. First, infrared active modes of the single chain having $\sigma$ polarization should be split in the crystal, with 

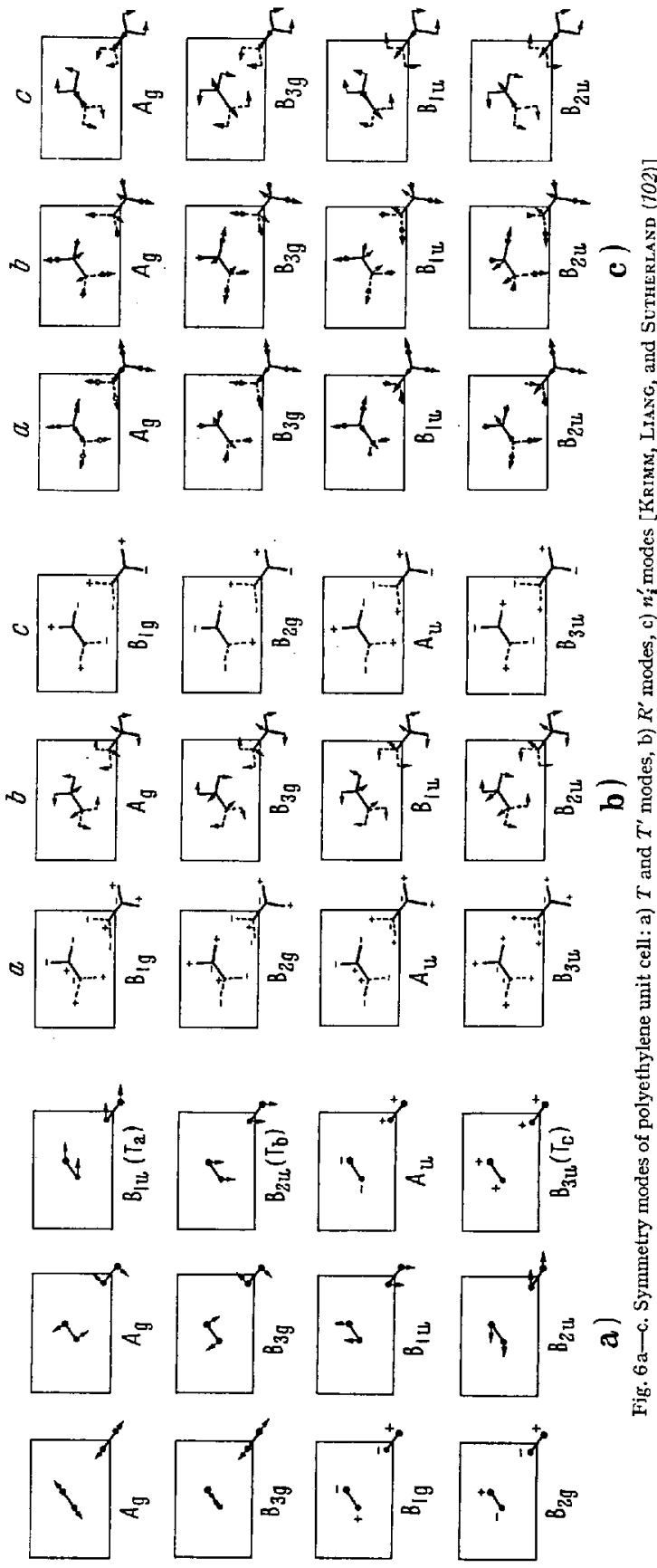

one component (the $B_{1 u}$ modes) polarized along the $a$-axis and the other (the $B_{2 u}$ modes) along the $b$-axis. The magnitude of the splitting will be, of course, a function of the details of the potential field and the intermolecular interaction, and cannot be predicted from symmetry considerations alone. Second, the $\pi \gamma_{w}\left(\mathrm{CH}_{2}\right)$ mode of the single chain is not split in the infrared spectrum of the crystal. Third, a $\gamma_{t}\left(\mathrm{CH}_{2}\right)$ mode, inactive in the single chain, is predicted to be active with $\pi$ polarization in the crystal. Fourth, two lattice modes, of species $B_{1 u}$ and $B_{2 u}$, should be infrared active. Fifth, although the above interaction splitting should also occur for the Raman active modes, it is likely that only one component will be observed. This is because for one of the components [e. g., the $\mathrm{B}_{3 j} \boldsymbol{v}_{+}(0)$ mode] the change in polarizability during the vibration is expected to be much smaller than for the other [the $A_{g} \nu_{+}(0)$ mode]. This is the most probable explanation for the lack of splitting actually 
found in the Raman spectrum [Nielsen and Woollett (160)]. On this basis we would predict the observed bands to be: $A_{g}-v_{s}\left(\mathrm{CH}_{2}\right), v_{a}\left(\mathrm{CH}_{2}\right)$, $\delta\left(\mathrm{CH}_{2}\right), \gamma_{r}\left(\mathrm{CH}_{2}\right), \boldsymbol{v}_{+}(0) ; \mathrm{B}_{2 g}-\gamma_{w}\left(\mathrm{CH}_{2}\right), \gamma_{t}\left(\mathrm{CH}_{2}\right), \boldsymbol{v}_{+}(\pi)$. Finally, it must be recalled that all of the restrictions will break down for the chains in the amorphous phase, where the symmetry is much lower.

The assignment of these modes can be assisted, as we have observed earlier, by normal vibration calculations for the molecule. Several such calculations have been done (see section III.A.2.), but the results are not entirely satisfactory. While the frequencies of some of the $\mathrm{CH}_{2}$ modes (viz., $\nu_{s}, v_{a}, \delta$, and $\gamma_{r}$ ) are predicted fairly consistently by all of the calculations, these differ significantly in their prediction of the other modes $\left(\gamma_{w}\right.$ and $\left.\gamma_{t}\right)$. They do, however, agree in the ordering of the $\mathrm{CH}_{2}$ deformation frequencies, viz., $\delta>\gamma_{w}>\gamma_{t}>\gamma_{r}$ (the skeletal stretching modes are generally placed between $\gamma_{t}$ and $\gamma_{r}$ ). This means that such computations can at present only serve as general guides to the assignments of the more difficult modes, viz., $\gamma_{w}$ and $\gamma_{t}$. These must be combined with studies on smaller $n$-paraffins [BRown, SHEPPARD, and Simpson (23); Tschamler (233); Nielsen and Holland (161)] and their single crystals [Krimm (93); Krimm, Liang, and Sutherland (102); Nielsen and Holland (167)], as well as Raman spectra, in order to obtain a satisfactory interpretation of the vibrational spectrum. We will consider briefly below the various arguments which lead to a satisfactory set of assignments.

Several bands are found in the spectrum of polyethylene which must be attributed to impurities formed during the polymerization process. In this category are bands at $\sim 600,908$, and $990 \mathrm{~cm}^{-1}$, due to $\mathrm{RCH}=\mathrm{CH}_{2}$ groups, the band at $888 \mathrm{~cm}^{-1}$, due to $\mathrm{RR}^{\prime} \mathrm{C}=\mathrm{CH}_{2}$ groups, and possibly the $964 \mathrm{~cm}^{-1}$ band, due to trans $\mathrm{RCH}=\mathrm{CHR}^{\prime}$ groups [BRown and SHEPPARD (21)], with the corresponding $v(\mathrm{C}=\mathrm{C})$ mode being found at $1640 \mathrm{~cm}^{-1}$. The presence of $\mathrm{C}=\mathrm{O}$ groups is indicated by bands at 1722 and $1737 \mathrm{~cm}^{-1}$ [RUGG, SMITH, and BACON (191)], and $\mathrm{OH}$ groups by the $3371 \mathrm{~cm}^{-1}$ band. Finally, $\mathrm{CH}_{3}$ groups are indicated by bands at 890 , $1375,1457,2874$, and $2960 \mathrm{~cm}^{-1}$ [RUGG, SMith, and WARTMAN (190)]. In some cases overtones of these bands are found also.

The infrared active $\nu_{a}\left(\mathrm{CH}_{2}\right), v_{s}\left(\mathrm{CH}_{2}\right), \delta\left(\mathrm{CH}_{2}\right)$, and $\gamma_{r}\left(\mathrm{CH}_{2}\right)$ fundamentals can be readily assigned as a result of the extensive spectroscopic studies on hydrocarbons which have been undertaken [SHEPPARD and Simpson (195)]. In addition, because of the polarized radiation studies on single crystals of normal paraffins [Krimm (93)], it is possible to assign uniquely the components of the doublets found in the spectrum for these bands to symmetry species. Similarly, the Raman active $\nu_{a}\left(\mathrm{CH}_{2}\right)$, $v_{s}\left(\mathrm{CH}_{2}\right), \delta\left(\mathrm{CH}_{2}\right), \nu_{+}(0)$, and $\nu_{+}(\pi)$ fundamentals can be unambiguously assigned, the latter two on the basis of normal vibration calculations 
(see section III. A. 2.). As we have noted, the Raman active fundamentals are not observed to be split, and the reasoning given in a preceding paragraph permits us to again make unique symmetry species assignments. These assignments are indicated in Table 5, and in Table 7, which lists the fundamental frequencies.

Table 7. Fundamental Frequencies of Polyethylene

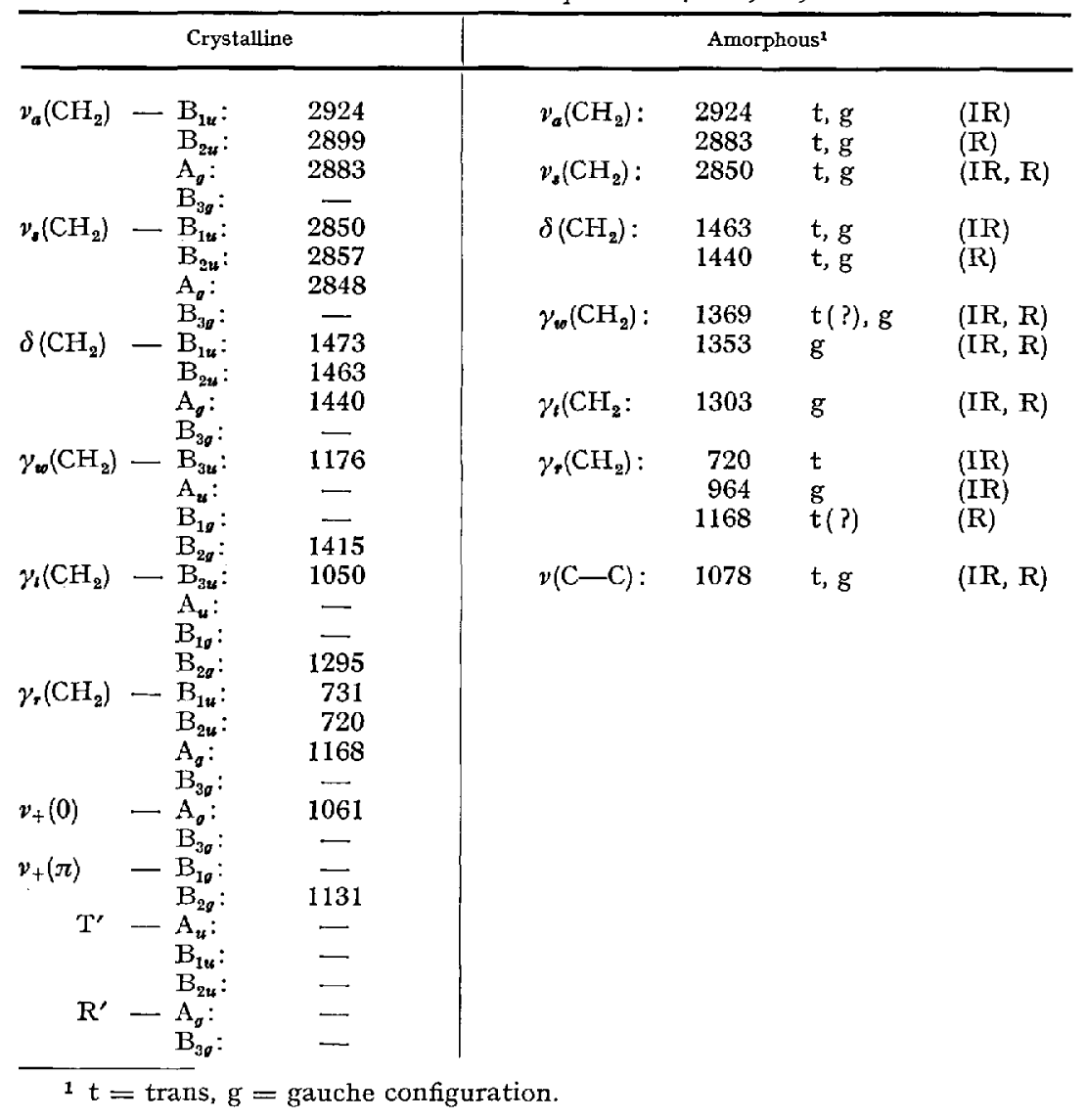

The remaining fundamentals are not as obviously assignable, but convincing arguments can now be presented. Consider the Raman fundamentals. On the basis of the normal mode calculations mentioned earlier, which seem to yield a consistent ordering of the frequencies, we would assign $\gamma_{w}\left(\mathrm{CH}_{2}\right), \gamma_{t}\left(\mathrm{CH}_{2}\right)$, and $\gamma_{r}\left(\mathrm{CH}_{2}\right)$ to the bands at 1415,1295 , and $1168 \mathrm{~cm}^{-1}$ respectively. This is also consistent [NIELSEN and Wooliet (160) ] with calculations of the expected relative intensities of these three bands [THeIMer (227)]. The assignments are made more secure by a 
consideration of combination bands. We have observed that in a single crystal of $\mathrm{n}-\mathrm{C}_{36} \mathrm{H}_{74}$ the band at $1885 \mathrm{~cm}^{-1}$ consists of a doublet, with components at about $1886 \mathrm{~cm}^{-1}$, polarized parallel to the $a$-axis, and $1875 \mathrm{~cm}^{-1}$, polarized parallel to the $b$-axis. Since these are $\sigma$ bands, i. e., of species $\mathrm{B}_{1 u}$ and $\mathrm{B}_{2 u}$, and obviously represent a combination between the $1168 \mathrm{~cm}^{-1}$ and $720,731 \mathrm{~cm}^{-1}$ bands, the $1168 \mathrm{~cm}^{-1}$ band must be of species $A_{g}$. Similarly, the $\pi\left(\mathrm{B}_{3 u}\right)$ band at $2016 \mathrm{~cm}^{-1}$ in crystalline n-paraffins can only arise as a combination between a $B_{2 g}$ mode at $1295 \mathrm{~cm}^{-1}$ and the $731 \mathrm{~cm}^{-1} \mathrm{~B}_{1 u}$ band. This automatically identifies the $1295 \mathrm{~cm}^{-1}$ band with $\gamma_{t}\left(\mathrm{CH}_{2}\right)$, and leaves the $1415 \mathrm{~cm}^{-1}$ band to be assigned to $\gamma_{w}\left(\mathrm{CH}_{2}\right)$.

The assignment of the $\gamma_{w}\left(\mathrm{CH}_{2}\right)\left(\mathrm{B}_{3 u}\right)$ and $\gamma_{t}\left(\mathrm{CH}_{2}\right)\left(\mathrm{B}_{3 u}\right)$ infrared active fundamentals of crystalline polyethylene has been the subject of continued investivation, concentrated primarily on the identification of the former mode. Most normal mode calculations predicted that the $\gamma_{w}\left(\mathrm{CH}_{2}\right)$ mode should occur near $1375 \mathrm{~cm}^{-1}$, and to begin with it was so assigned [Simanouti and Mizushima (200); Kellner (87)]. Other investigations [Fox and Martin (56); Cross, Richards, and Willis $(42 a)$ ], however, seemed to have established with a fair degree of certainty that this band is due to a $\mathrm{CH}_{3}$ group mode, and this is supported by more recent studies [Bryant and Voter (25); Slowisski, Walter, and Miller (202); Willbourn (243)]. On this basis the $\gamma_{w}\left(\mathrm{CH}_{2}\right)$ mode was therefore assigned to the $\pi$ band at $1303 \mathrm{~cm}^{-1}$ [BRown, Sheppard, and Simpson (22)]. Subsequent studies [KRimm (94); Nichols, (158); Krimm, Liang, and SutherLand (102)] showed that the $1303 \mathrm{~cm}^{-1}$ band was associated only with the amorphous regions, so its assignment to $\gamma_{w}\left(\mathrm{CH}_{2}\right)$ in crystalline polyethylene had to be discarded. Studies on n-paraffin single crystals [Krimm, Liang, and Sutherland (102)] indicated the presence of a weak $\pi$ band at $1369 \mathrm{~cm}^{-1}$, where a band, presumably due to $\mathrm{CH}_{2}$ groups [CRoss, Richards, and Willis (42a)], was already known to be present in amorphous polyethylene and melted paraffins. Coupled with the presence of a band at this position in terminally deuterated n-octane [Pimentel and Klemperer (172)], the evidence seemed to indicate the assignment of $\gamma_{w}\left(\mathrm{CH}_{2}\right)$ to the $1369 \mathrm{~cm}^{-1}$ band, although its increase in intensity upon melting in the paraffins remained puzzling. Recent studies [NIELsEn and Holland (161)] have emphasized more clearly the various reasons for holding this assignment to be not completely satisfactory, and it seems necessary to reconsider it now in the light of the new data which are available.

A more satisfactory assignment for $\gamma_{w}\left(\mathrm{CH}_{2}\right)\left(\mathrm{B}_{3 u}\right)$, as well as $\gamma_{t}\left(\mathrm{CH}_{2}\right)$ $\left(\mathrm{B}_{3 u}\right)$, seems to emerge from consideration of the symmetry species of the Raman active fundamentals, the infrared combination bands and their polarization, and the bands which seem to be clearly associated with the 
crystalline phase of polyethylene. These point to the assignment of the 1050 and $1176 \mathrm{~cm}^{-1} \pi, \mathrm{C}$ bands as $\mathrm{B}_{3 u}$ fundamentals, the former probably to $\gamma_{t}\left(\mathrm{CH}_{2}\right)$ and the latter to $\gamma_{w}\left(\mathrm{CH}_{2}\right)$ (there may, of course, be mixing of these two in the actual normal modes giving rise to these bands). This interpretation is supported by the observation that the assumption of $1369 \mathrm{~cm}^{-1}\left(\mathrm{~B}_{3 u}\right)$ will only account for two binary combination bands (at 2525 and $2658 \mathrm{~cm}^{-1}$ ), both of which can equally well be ascribed to combinations involving amorphous chain frequencies, while the assumption of $1050 \mathrm{~cm}^{-1}\left(\mathrm{~B}_{3 u}\right)$ and $1176 \mathrm{~cm}^{-1}\left(\mathrm{~B}_{3 u}\right)$ satisfactorily accounts for six binary combinations (at 2242, 2275, 2345, 2473, 2597, and $2619 \mathrm{~cm}^{-1}$ ), as well as ten ternary combinations. The ranges which would result for the various frequencies, viz., $\gamma_{r}\left(\mathrm{CH}_{2}\right): 720-1168 \mathrm{~cm}^{-1}$, $\gamma_{t}\left(\mathrm{CH}_{2}\right): 1050-1295$, and $\gamma_{w}\left(\mathrm{CH}_{2}\right): 1176-1415 \mathrm{~cm}^{-1}$, are in line with predictions based on studies of the spectra of a series of n-paraffins [BRown, Sheppard, and Simpson (23); Tschamler (233); Sheppard (194a)], although a bit larger. The assignment of $\gamma_{w}\left(\mathrm{CH}_{2}\right)\left(\mathrm{B}_{3 u}\right)$ to the $1176 \mathrm{~cm}^{-1}$ band is also in agreement with a recent study of the $\mathrm{CH}_{2}$ wagging modes in the entire series of normal paraffins from $\mathrm{C}_{20} \mathrm{H}_{42}$ through $\mathrm{C}_{30} \mathrm{H}_{62}[\operatorname{SNYDER}(205)]$. When combined with the other fundamental assignments a satisfactory accounting of most combination bands is provided (see Table 5). It is interesting to note that, with these assignments, the highest frequency of each of the internal $\mathrm{CH}_{2}$ modes $\left(v_{a}, v_{s}\right.$, and $\left.\delta\right)$ is infrared active, with a relatively small gap between infrared- and Raman-active fundamentals, whereas the highest frequency of each of the external $\mathrm{CH}_{2}$ modes $\left(\gamma_{w}, \gamma_{t}\right.$, and $\gamma_{r}$ ) is Raman active and there is a relatively large frequency gap between infrared and Raman-active fundamentals.

Since a polyethylene spectrum in general represents a superposition of crystalline and amorphous spectra, it is of interest to determine the fundamental frequencies of the non-crystalline chains. The data are easy to obtain, viz., from melted polyethylene or n-paraffins, but a detailed interpretation may be difficult because of the many structural species which can exist in the amorphous state. In general we can say that not only do we expect inter-chain interactions to disappear in the noncrystalline state, but it is also likely that intra-chain interactions, which in a long planar zig-zag chain give rise to widely separated infrared and Raman active modes, will diminish and the frequencies will tend to approach those of isolated $\mathrm{CH}_{2}$ groups, e. g., such as those in propane. Of course, the $\mathrm{CH}_{2}$ groups will not behave as if they were completely isolated; for example, the frequencies of such groups will undoubtedly depend upon whether they are in a trans or gauche conformation with respect to neighboring $\mathrm{CH}_{2}$ groups. From the detailed theoretical analysis of 1,2-dichloroethane [Mrzushima (137)], and the experimental studies of this molecule in the solid and liquid states [BROwN and 
SHEPPARD (20)], we have a fair idea of the frequencies, and their pattern, to be associated with trans and gauche configurations of $\mathrm{CH}_{2}-\mathrm{CH}_{2}$ units. These studies suggest the assignment of fundamental frequencies of the amorphous polyethylene chains shown in Table 7. The $964 \mathrm{~cm}^{-1}$ band is found to intensify when paraffins are melted [KRIMM, LIANG, and SutherLAND (102)]; this means that not all of this band in polyethylene can be due to $\mathrm{RCH}=\mathrm{CHR}$ groups. By analogy with the infrared band which develops at $944 \mathrm{~cm}^{-1}$ in liquid 1,2-dichloroethane, the $964 \mathrm{~cm}^{-1}$ band in polyethylene may be due to $\gamma_{r}\left(\mathrm{CH}_{2}\right)$ for amorphous chains in which the gauche configuration is present. Again, by analogy with the two strong $\gamma_{w}\left(\mathrm{CH}_{2}\right)$ infrared bands at 1312 and $1286 \mathrm{~cm}^{-1}$ and the weak single $1143 \mathrm{~cm}^{-1} \gamma_{t}\left(\mathrm{CH}_{2}\right)$ band in liquid 1,2-dichloroethane we assign the 1369 and $1353 \mathrm{~cm}^{-1}$ bands to $\gamma_{w}\left(\mathrm{CH}_{2}\right)$ modes and the $1303 \mathrm{~cm}^{-1}$ band to the $\gamma_{t}\left(\mathrm{CH}_{2}\right)$ mode of gauche $\mathrm{CH}_{2}-\mathrm{CH}_{2}$ units. The $1369 \mathrm{~cm}^{-1}$ band, which is found to occur weakly in solid n-paraffins, may also contain a contribution from trans structures. This would be consistent with the somewhat higher dichroism of this band, as well as its relatively smaller intensification upon melting as compared with the $1353 \mathrm{~cm}^{-1}$ band. The assignment of the skeletal stretching mode at $1078 \mathrm{~cm}^{-1}$ is based on studies of the melting of n-paraffin crystals [Krimm, LiaNG, and SutherLaND (102)]. The probable presence of bands in the Raman spectrum is inferred from studies of the Raman spectra of liquid n-paraffins [Mrzushima and Simanouti (138)]. While these assignments cannot be considered completely unambiguous, they seem reasonable and they do provide a satisfactory explanation for some of the combination bands.

As for the remaining bands, some seem to be Raman active fundamentals which appear weakly in the infrared spectrum, perhaps as a result of end effects, such as short portions of planar zig-zag chain in the amorphous regions. Infrared bands at 1065, 1131, 1168, and $1436 \mathrm{~cm}^{-1}$ may belong in this category. The origin of the low frequency bands is unknown; some may arise from impurities or from the $\mathrm{B}_{1 u}$ and $\mathrm{B}_{2 u}$ translatory modes of the lattice. There is as yet no unambiguous assignment of the latter. A few weak bands in the $1100-1200 \mathrm{~cm}^{-1}$ region are unassigned.

One aspect of the spectrum that has received considerable attention but is as yet not completely understood is the splitting in the $\sigma$ fundamentals of a single chain which gives rise to the $\mathrm{B}_{1 u}$ and $\mathrm{B}_{2 u}$ fundamentals of the crystal. From studies on polyethylene and n-paraffins it was concluded [STEIN and SUTHERLAND $(207,208)$ ] that this splitting arises from interaction between the two chains in the unit cell. As we have seen, such a splitting is predicted from a group theory analysis of the spectrum of crystalline polyethylene, and the predicted dichroic properties of the components are verified by studies on n-paraffin single crystals [KRIMM 
(93)]. Other data support the qualitative nature of this explanation: the splitting (observed for the $\gamma_{r}\left(\mathrm{CH}_{2}\right)$ modes) disappears in solid solutions of normal and deuterated n-paraffins [KRIMM, LIANG, and SuTHERLAND (102)], and the doublet is replaced by a singlet in those n-paraffins which crystallize in a triclinic structure [SNYder (204); Chapman (32); Martin, Johnston, and O'NeAL (125)]. In both cases we would predict the absence of an interaction, in the former because the corresponding frequencies on neighboring chains are now widely separated and in the latter because there is only a single chain per unit cell, and the consequent absence of splitting is well verified. A satisfactory quantitative theory for this doubling, however, remains to be developed. A theory based on simple van der Waals interactions between hydrogen atoms on adjacent chains [STEIN (206)] is in disagreement with the data on two main points: it predicts that the high frequency component of the doublet should be polarized along the $a$-axis of the crystal for the $\gamma_{r}\left(\mathrm{CH}_{2}\right)$ mode (as is found) and along the $b$-axis for the $\delta\left(\mathrm{CH}_{2}\right)$ mode (contrary to observations); and it predicts that the two components should be at frequencies higher than that for the unperturbed chain, whereas the indication from the work on solid solutions [KRIMM, LIANG, and SUTHERLAND (102)] is that the components of the doublet bracket the unperturbed frequency. These facts indicate therefore that the present theory is either too over-simplified or perhaps does not include significant aspects of the interaction potential. For example, it may well be that induced dipole effects are most significant in bringing about the splitting. It is to be hoped that this aspect of the infrared spectrum will be investigated further in greater detail, since it not only will provide a better understanding of the vibrational spectrum but will furnish further insight into the nature of intermolecular forces between long chain molecules.

This discussion of the spectrum of polyethylene indicates the depth of understanding that it is possible to obtain as a result of the concerted application of the methods of analysis discussed in Part III. This does not mean that all problems have been solved: if the present assignments are correct, then the predictions of most normal vibration analyses with respect to the $\mathrm{B}_{3 u} \gamma_{w}\left(\mathrm{CH}_{2}\right)$ and $\gamma_{i}\left(\mathrm{CH}_{2}\right)$ modes must be re-examined; a better theory is needed, as we have seen, to explain the interaction splitting of the $\sigma$ modes in the crystalline state; additional evidence on the fundamentals of the amorphous chain is desirable; assignments of the translatory and rotatory crystalline chain modes deserve study (probably through the investigation of the low frequency region of deutero-paraffins); band intensity changes associated with the crystalline to amorphous transition need elucidation. Nevertheless we can say with some confidence that a basic understanding of the polyethylene spectrum 
has been achieved. This knowledge underlies the use of the infrared spectrum in the study of various properties of the polymer, such as $\mathrm{CH}_{3}$ group content, crystallinity, orientation of crystalline and amorphous regions, and changes on oxidation [see Schnell (193), for references to these studies].

\section{B. Polytetrafluoroethylene}

Polytetrafluoroethylene (PTFE) has a chemical structure which can be designated by $\left.{ }_{-1} \mathrm{CF}_{2}\right)_{\bar{n}}$. From its resemblance to the chemical structure of polyethylene it might be thought that the spectra of these two polymers should be quite similar. They do in fact resemble each other, but there are also important differences. This is a consequence of the fact that the PTFE chain configuration is quite different from that of polyethylene, and also the intramolecular forces are undoubtedly significantly different in the two cases. As we shall see, the spectrum is moderately well understood, but not in quite as great detail as that of polyethylene. This is primarily a result of the lack of Raman data on the polymer and certain key polarization data in the infrared.

The spectrum of a stretched PTFE specimen is shown in Fig. 7 [LIANG and KRIMM (111)], covering the range from 70 to $3300 \mathrm{~cm}^{-1}$, with polarization data at frequencies above $350 \mathrm{~cm}^{-1}$. Reflection spectra have been obtained in the 1000 to $1600 \mathrm{~cm}^{-1}$. region [ROBINSON and PRICE (187)], and the spectrum has been recorded up to frequencies of about $3700 \mathrm{~cm}^{-1}$ in a recent study [MoyniHan (141)]. The band descriptions are listed in Table 8 together with the assignments, which will be discussed below. (In some cases the band positions represent mean values of those quoted in Liang and

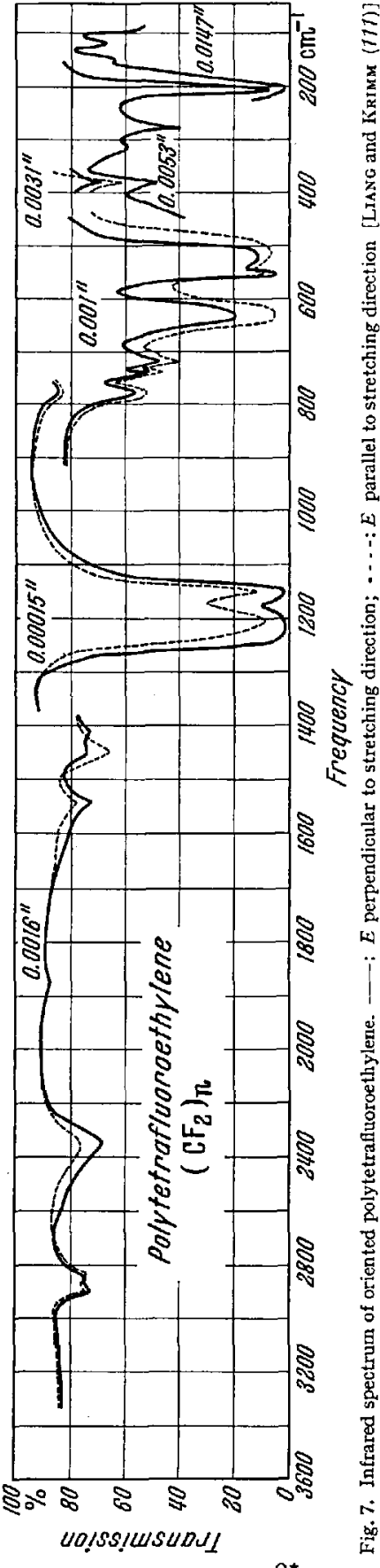


Table 8. Infrared Spectrum of Polytetrafluoroethylene

\begin{tabular}{|c|c|c|c|c|}
\hline \multirow{2}{*}{$\begin{array}{l}\text { Frequency, } \\
\mathrm{cm}^{-1}\end{array}$} & \multicolumn{3}{|c|}{ Description } & \multirow{2}{*}{ Assignment } \\
\hline & R. I. & Pol'n & Phase & \\
\hline 102 & $\mathrm{mw}$ & & & \\
\hline 124 & w & & & \\
\hline 149 & $\mathrm{w}$ & & & \\
\hline 203 & s & $\sigma$ & & $\gamma_{r}\left(\mathrm{CF}_{2}\right)\left(\mathrm{E}_{1}\right)$ \\
\hline 277 & m & & & $\gamma_{w}\left(\mathrm{CF}_{2}\right)\left(\mathrm{E}_{1}\right)$ \\
\hline 321 & $\mathrm{w}$ & & & $\gamma_{i}\left(\mathrm{CF}_{2}\right)\left(\mathrm{E}_{\mathrm{J}}\right)$ \\
\hline 384 & $\mathbf{m}$ & $\pi$ & A & \\
\hline 516 & vs & $\pi$ & $\mathrm{C}$ & $\gamma_{r}\left(C_{2}\right)\left(A_{2}\right)$ \\
\hline 553 & $\mathbf{s}$ & $\sigma$ & C & $\delta\left(\mathrm{CF}_{2}\right)\left(\mathrm{E}_{1}\right)$ \\
\hline 625 & $\mathrm{~s}$ & $\pi$ & $\mathrm{C}$ & $\gamma_{10}\left(C F_{2}\right)\left(A_{2}\right)_{15}$ \\
\hline 638 & $\mathbf{s}$ & $\pi$ & C & $\gamma_{20}\left(\mathrm{CF}_{2}\right)\left(\mathrm{A}_{2}\right)_{13}$ \\
\hline 703 & $\mathrm{vw}$ & & A & \\
\hline 720 & $\mathrm{~m}$ & $\pi$ & A & \\
\hline 740 & $\mathrm{~m}$ & $\pi$ & A & \\
\hline 780 & $\mathrm{~m}$ & $\pi$ & A & \\
\hline 850 & $w$ & & A & \\
\hline 932 & $\mathrm{w}$ & $\sigma$ & c & $\delta\left(\mathrm{A}_{1}\right)+\gamma_{r}\left(\mathrm{E}_{1}\right)=933\left(\mathrm{E}_{1}\right)$ \\
\hline 1152 & vvs & $\sigma$ & c & $v_{s}\left(\mathrm{CF}_{2}\right)\left(\mathrm{E}_{1}\right)$ \\
\hline 1210 & vvs & $\pi ?$ & c & $v_{a}\left(\mathrm{CF}_{2}\right)\left(\mathrm{A}_{2}\right)$ \\
\hline 1242 & vvs & $\sigma$ & C & $v_{a}\left(\mathrm{CF}_{2}\right)\left(\mathrm{E}_{1}\right)$ \\
\hline 1415 & m & $\cdot \sigma$ & C & $v_{a}\left(\mathrm{~A}_{2}\right)+\gamma_{r}\left(\mathrm{E}_{1}\right)=1413\left(\mathrm{E}_{1}\right)$ \\
\hline 1450 & m & $\pi$ & $\mathrm{C}$ & $v_{a}\left(E_{1}\right)+\gamma_{r}\left(E_{1}\right)=1445\left(A_{2}\right)$ \\
\hline 1545 & $\mathrm{~m}$ & $\sigma$ & $\mathrm{C}$ & $v_{a}\left(\mathrm{E}_{1}\right)+\gamma_{b}\left(\mathrm{~A}_{1}\right)=1549\left(\mathrm{E}_{1}\right)$ \\
\hline 1703 & $w$ & $\sigma$ & C & $v_{s}\left(\mathrm{~A}_{1}\right)+\gamma_{t}\left(\mathrm{E}_{1}\right)=1701\left(\mathrm{E}_{1}\right)$ \\
\hline 1732 & $\mathrm{w}$ & $\sigma$ & $\mathrm{C}$ & $2 \times \delta\left(\mathrm{A}_{1}\right)+\gamma_{v}\left(\mathrm{E}_{1}\right)=1737\left(\mathrm{E}_{1}\right)$ \\
\hline 1792 & $\mathrm{~m}$ & $\sigma$ & $\mathrm{C}$ & $v_{s}\left(\mathrm{E}_{1}\right)+\gamma_{t o}\left(\mathrm{~A}_{2}\right)_{13}=1790\left(\mathrm{E}_{1}\right)$ \\
\hline 1859 & w & $\sigma$ & $\mathrm{C}$ & $\left\{\begin{array}{l}v_{a}\left(\mathrm{E}_{1}\right)+\nu(\mathrm{CC})\left(\mathrm{A}_{1}\right)=1849\left(\mathrm{E}_{1}\right) \\
v_{a}\left(\mathrm{E}_{1}\right)+\gamma_{w}\left(\mathrm{~A}_{2}\right)_{15}=1867\left(\mathrm{E}_{1}\right)\end{array}\right.$ \\
\hline 1883 & $\mathrm{w}$ & $\sigma$ & $\mathrm{C}$ & $\left\{\begin{array}{l}v_{a}\left(\mathrm{E}_{1}\right)+\gamma_{o}\left(\mathrm{~A}_{2}\right)_{13}=1880\left(\mathrm{E}_{1}\right) \\
v_{1}\left(\mathrm{E}_{1}\right)+\delta\left(\mathrm{A}_{1}\right)=182\left(\mathrm{E}_{1}\right)\end{array}\right.$ \\
\hline 1935 & $\mathrm{w}$ & $\sigma$ & C & $v_{a}\left(\mathrm{~A}_{1}\right)+\delta\left(\mathrm{E}_{1}\right)=1933\left(\mathrm{E}_{1}\right)$ \\
\hline 1974 & $\mathrm{vw}$ & $\sigma$ & $\mathrm{C}$ & $v_{a}\left(\mathrm{E}_{1}\right)+\delta\left(\mathrm{A}_{1}\right)=1972\left(\mathrm{E}_{1}\right)$ \\
\hline 2300 & $\mathrm{vw}$ & & $\mathrm{C}$ & $v_{a}\left(\mathrm{E}_{1}\right)+\delta\left(\mathrm{A}_{1}\right)+\gamma_{l}\left(\mathrm{E}_{1}\right)=2293\left(\mathrm{~A}_{2}\right) ?$ \\
\hline 2365 & $\mathrm{w}$ & $\sigma$ & $\mathrm{C}$ & $v_{a}\left(\mathrm{~A}_{2}\right)+v_{s}\left(\mathrm{E}_{1}\right)=2362\left(\mathrm{E}_{1}\right)$ \\
\hline 2390 & $\mathrm{~m}$ & $\pi$ & C & $v_{a}\left(\mathrm{E}_{1}\right)+v_{a}\left(\mathrm{E}_{1}\right)=2394\left(\mathrm{~A}_{2}\right)$ \\
\hline 2450 & $\mathrm{vw}$ & & $\mathrm{C}$ & $v_{a}\left(\mathrm{E}_{1}\right)+v_{a}\left(\mathrm{~A}_{2}\right)=2452\left(\mathrm{E}_{1}\right)$ \\
\hline 2530 & vw & $\sigma$ & C & $v_{z}\left(\mathrm{E}_{1}\right)+v_{s}\left(\mathrm{~A}_{1}\right)=2532\left(\mathrm{E}_{1}\right)$ \\
\hline 2590 & $\mathrm{vw}$ & & $\mathrm{C}$ & $\nu_{a}\left(\mathrm{~A}_{2}\right)+\nu_{s}\left(\mathrm{~A}_{1}\right)=2590\left(\mathrm{~A}_{2}\right)$ \\
\hline 2620 & $\mathrm{vw}$ & & $\mathrm{C}$ & $v_{a}\left(\mathrm{E}_{1}\right)+v_{s}\left(\mathrm{~A}_{1}\right)=2622\left(\mathrm{E}_{1}\right)$ \\
\hline 2670 & vw & $\sigma$ & $\mathrm{C}$ & $v_{s}\left(\mathrm{E}_{1}\right)+v_{a}\left(\mathrm{~A}_{2}\right)+v_{t}\left(\mathrm{~A}_{1}\right)=2669\left(\mathrm{E}_{1}\right)$ \\
\hline 2850 & $\mathrm{vw}$ & $\pi$ & C & $v_{d}\left(\Lambda_{1}\right)+v_{t}\left(\mathrm{E}_{1}\right)+\gamma_{t}\left(\mathrm{E}_{1}\right)=2853\left(\mathrm{~A}_{2}\right)$ \\
\hline 2925 & $\mathrm{vW}$ & $\sigma$ & C & $v_{d}\left(\mathrm{~A}_{1}\right)+v_{a}\left(\mathrm{E}_{1}\right)+\gamma_{t}\left(\mathrm{~A}_{1}\right)=2929\left(\mathrm{E}_{1}\right)$ \\
\hline 3090 & $\mathrm{vw}$ & & $\mathrm{C}$ & $v_{s}\left(\mathrm{E}_{1}\right)+v_{a}\left(\mathrm{~A}_{2}\right)+\delta\left(\mathrm{A}_{1}\right)=3092\left(\mathrm{E}_{1}\right)$ \\
\hline 3450 & $\mathrm{vw}$ & & C & $3 \times v_{s}\left(\mathrm{E}_{1}\right)=3456\left(\mathrm{E}_{1}\right)$ \\
\hline 3570 & vw & & C & $\nu_{s}\left(\mathrm{E}_{1}\right)+2 \times \nu_{a}\left(\mathrm{~A}_{2}\right)=3572\left(\mathrm{E}_{1}\right)$ \\
\hline 3670 & $\mathrm{vw}$ & & $\mathrm{C}$ & $v_{a}\left(\mathrm{E}_{1}\right)+2 \times v_{a}\left(\mathrm{~A}_{2}\right)=3662\left(\mathrm{E}_{1}\right)$ \\
\hline
\end{tabular}

Krimm (111) and Moynihan (141)]. Data on the amorphous origin of some of the bands were taken from several studies [Miller and Willis (133); Pokrovsky and Kotova (177); Moynihan (141)]. The remaining bands are assumed to arise from the crystalline material. No 
Raman spectrum of PTFE has been obtained as yet, so that an attempt has been made to infer the Raman active fundamentals from data on $\mathrm{n}-\mathrm{C}_{7} \mathrm{~F}_{16}$ [A. P. I. Spectra (7), No. 196] and from combination bands [Moynihan (141)]. These, as well as the infrared, fundamentals are shown in Table 9.

The basic crystalline chain structure of PTFE has been worked out from x-ray diffraction studies [BUNN and HowElls (29)]. It consists of a helical arrangement of $\mathrm{CF}_{2}$ groups along the chain with $13 \mathrm{CF}_{2}$ groups in the identity period of the helix (see Fig. 8). The two-fold axis of each $\mathrm{CF}_{2}$ group is perpendicular to the helix axis. The polymer undergoes a phase transition at $19^{\circ} \mathrm{C}$. which seems to be accompanied by a change in the identity period, there now being $15 \mathrm{CF}_{2}$ groups in the repeat [PIERCE,

Table 9. Fundamental Frequencies of Polytetrafluoroethylene: $A_{1}, A_{2}$, and $E_{1}$ Species

\begin{tabular}{|c|c|c|}
\hline \multirow[t]{4}{*}{$A_{1}$} & $v(\mathrm{CC}):$ & 607 \\
\hline & $v_{1}\left(\mathrm{CF}_{2}\right):$ & 1380 \\
\hline & $\delta\left(\mathrm{CF}_{2}\right):$ & 730 \\
\hline & $\gamma_{t}\left(\mathrm{CF}_{2}\right):$ & 307 \\
\hline \multirow[t]{3}{*}{$A_{2}$} & $\nu_{a}\left(\mathrm{CF}_{2}\right):$ & 1210 \\
\hline & $\gamma_{w}\left(\mathrm{CF}_{2}\right):$ & 638 \\
\hline & $\gamma_{r}\left(\mathrm{CF}_{2}\right):$ & 516 \\
\hline \multirow{6}{*}{$E_{1}$} & $\begin{array}{l}v(\mathrm{CC}): \\
v(\mathrm{CF}):\end{array}$ & 1242 \\
\hline & $v,\left(\mathrm{CF}_{2}\right):$ & 1152 \\
\hline & $\delta\left(\mathrm{CF}_{2}\right):$ & 553 \\
\hline & $\gamma_{w}\left(\mathrm{CF}_{2}\right):$ & 277 \\
\hline & $\gamma_{1}\left(\mathrm{CF}_{2}\right):$ & 321 \\
\hline & $\gamma_{r}\left(\mathrm{CF}_{2}\right):$ & 203 \\
\hline
\end{tabular}
Clark, WhitNey, and Bryant (171)]. In both cases there is one chain per unit cell, which is pseudo-hexagonal below $19^{\circ}$ and hexagonal above this temperature. The symmetry analysis, therefore, need only deal with

a)

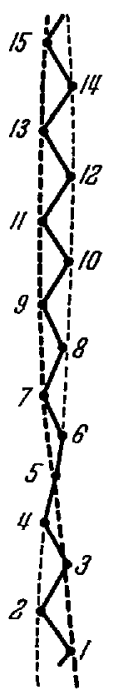

b)

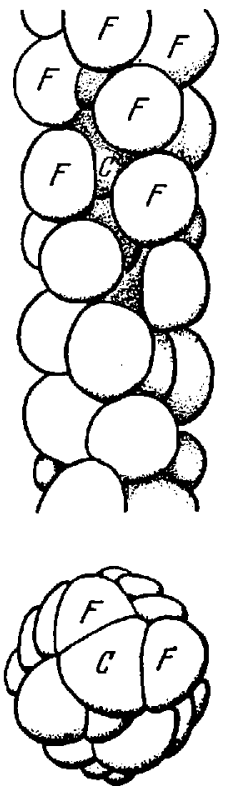

c)

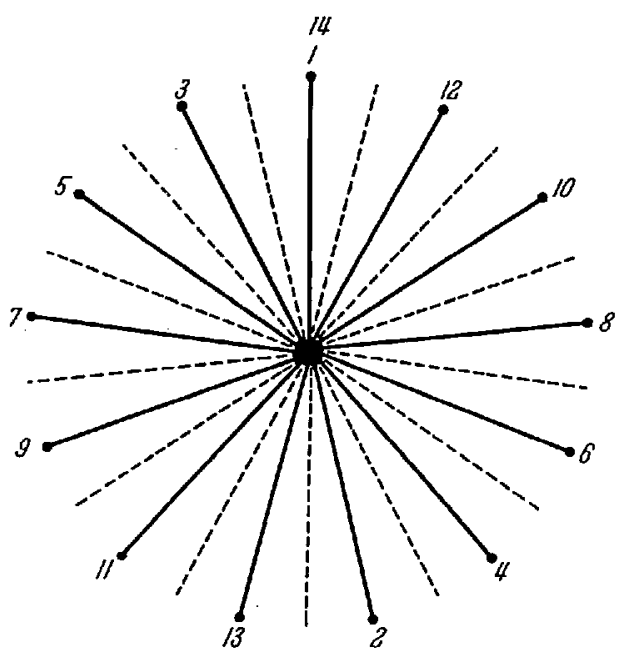

Fig. 8a-c. Structure of polytetrafluoroethylene. a) Twisted carbon backbone. b) Side and end views of molecule. c) Symmetry relations between $\mathrm{CF}_{2}$ groups [LiANG and Ккімм (117)] 
a single chain. The original symmetry analysis [LIANG and KRIMM (117)] was done on the chain with $13 \mathrm{CF}_{2}$ groups in the identity period. The results for a chain with $15 \mathrm{CF}_{2}$ groups are the same with respect to the infrared and Raman active frequencies [MoYNiHAN (141)]. These predictions, both for the active fundamentals and for combinations and overtones, are shown in Table 10. A set of symmetry modes has beer

Table 10. Results of Symmetry Analysis of Polytetrafluoroethylene

\begin{tabular}{l|l|l|l|l}
\hline & Species & $\begin{array}{c}\text { No. of inter- } \\
\text { nal modes }\end{array}$ & Activity & Pol'n \\
\hline \multirow{3}{*}{ Fundamentals: } & $\mathrm{A}_{1}$ & 4 & $\mathrm{R}$ & \\
& $\mathrm{A}_{2}$ & 4 & $\mathrm{IR}$ & $\pi$ \\
& $\mathrm{E}_{1}$ & 7 & $\mathrm{IR}, \mathrm{R}$ & $\sigma$ \\
& $\mathrm{E}_{2}$ & 9 & $\mathrm{R}$ & \\
Overtones: & $\mathrm{E}_{3}-\mathrm{E}_{8}\left(\mathrm{E}_{2}\right)$ & $36(45)$ & - & \\
& $\mathrm{A}_{1}^{2}=\mathrm{A}_{1}$ & & $\mathrm{R}$ & \\
& $\mathrm{A}_{2}^{2}=\mathrm{A}_{1}$ & $\mathrm{R}$ & \\
& $\mathrm{E}_{1}^{2}=\mathrm{A}_{1}+\mathrm{E}_{2}$ & & $\mathrm{R}$ & \\
& $\mathrm{E}_{1}^{3}=\mathrm{E}_{1}$ & & $\mathrm{IR}$ & $\sigma$ \\
& $\mathrm{A}_{1} \times \mathrm{A}_{1}=\mathrm{A}_{1}$ & & $\mathrm{R}$ & \\
& $\mathrm{A}_{1} \times \mathrm{A}_{2}=\mathrm{A}_{2}$ & & $\mathrm{IR}$ & $\pi$ \\
& $\mathrm{A}_{1} \times \mathrm{E}_{1}=\mathrm{E}_{1}$ & & $\mathrm{R}, \mathrm{IR}$ & $\sigma$ \\
& $\mathrm{A}_{2} \times \mathrm{A}_{2}=\mathrm{A}_{1}$ & & $\mathrm{R}, \mathrm{IR}$ & $\sigma$ \\
& $\mathrm{A}_{2} \times \mathrm{E}_{1}=\mathrm{E}_{1}$ & & $\mathrm{R}, \mathrm{IR}$ & $\pi$
\end{tabular}

given for the helical PTFE chain [LiAng and Krimm (111)]. These can be described in terms of the modes of an individual $\mathrm{CF}_{2}$ group and the phase relation between the vibrations in neighboring groups. In the $A_{1}$ and $A_{2}$ modes the vibrations in neighboring $\mathrm{CF}_{2}$ groups are in phase, while in the $\mathrm{E}_{1}$ modes they differ by $\pm \frac{14 \pi}{13}$ (or $\pm \frac{16 \pi}{15}$ for a helix with $15 \mathrm{CF}_{2}$ groups in the repeat) and in the $\mathrm{E}_{2}$ modes by $\pm 2 \cdot \frac{14 \pi}{13}$ (or $\pm 2 \cdot \frac{16 \pi}{15}$ ).

In making the assignments several guiding principles have been followed. The frequencies of PTFE have been assumed to be close to the corresponding fundamentals of a hypothetical planar zig-zag - $\left(\mathrm{CF}_{2}\right)_{n}$ chain. These have been calculated [LIANG and KRIMM (111)], and serve as a general guide for assigning bands even though some of the particular frequency values must probably be accepted with caution. By analogy with the discussion in the previous section, the $A_{1}$ Raman fundamentals are probably the strongest, and therefore only fundamentals and combinations involving $A_{1}$ Raman modes have been considered. The assignment of combination and overtone bands is of help in assigning some of the fundamentals, particularly those in the $A_{1}$ and $A_{2}$ species. For the assignment of most of the modes we depend upon the dichroism of the bands in the infrared spectrum. 
The group of strong bands at $1150-1250 \mathrm{~cm}^{-1}$ is undoubtedly associated with the $\nu\left(\mathrm{CF}_{2}\right)$ modes. As has been pointed out [MoYNiHAN (141)], the higher frequency band is actually a doublet with components at about 1210 and $1242 \mathrm{~cm}^{-1}$. While the latter is clearly a $\sigma$ band, the evidence on the polarization of the former is not entirely certain. Polarized spectra of thin films (e. g., see Fig. 7) indicate that this band may be $\pi$, but the film was still too thick to be certain. Other studies [Moyninas (141)] were equally uncertain. This band is assumed to be $\pi$ on the basis of the tentative polarization data and the agreement which is obtained with the dichroism of several combination bands. A firmer experimental basis for the dichroism of the $1210 \mathrm{~cm}^{-1}$ band is obviously needed. With the above assumption, the $A_{2}$ and $E_{1} \nu\left(C_{2}\right)$ modes are readily assigned. The $\mathrm{A}_{1} v\left(\mathrm{CF}_{2}\right)$ mode is assigned to a Raman active band at about $1380 \mathrm{~cm}^{-1}$ on the basis of a) the indication, from combination bands, of an $A_{1}$ mode near this frequency, $b$ ) the presence of a moderately strong Raman band at $1375 \mathrm{~cm}^{-1}$ in $\mathrm{n}-\mathrm{C}_{7} \mathrm{~F}_{16}$, and c) the indication from the study of perfluorocyclobutane [CLAASEN (34)] that Raman active $\nu\left(\mathrm{CF}_{2}\right)$ frequencies occur as high as $1430 \mathrm{~cm}^{-1}$. From its $\pi$ polarization, and the position predicted in a planar zig-zag chain, the $638 \mathrm{~cm}^{-1}$ band can be assigned to $\gamma_{w}\left(\mathrm{CF}_{2}\right)$ $\left(A_{2}\right)$. The appearance of this mode at $625 \mathrm{~cm}^{-1}$ in the chain containing $15 \mathrm{CF}_{2}$ groups per repeat is consistent with the probable sensitivity of this mode to the twist in the carbon chain backbone. The remaining $A_{2}$ mode must be assigned to the only other strong $\pi$ band, viz., that at $516 \mathrm{~cm}^{-1}$. The other $A_{1}$ modes are assigned on the basis of their presence in the Raman spectrum of $n-C_{7} F_{16}$ and agreement with combination bands, although the exact assignment is not certain. The strongest band in the Raman spectrum of $\mathrm{n}_{-}-\mathrm{C}_{7} \mathrm{~F}_{16}$ is at $754 \mathrm{~cm}^{-1}$ (relative intensity 100 compared to 15 for the next strongest bands), so that an $A_{1}$ mode at $730 \mathrm{~cm}^{-1}$ is not unexpected. Its assignment to $\delta\left(\mathrm{CF}_{2}\right)\left(\mathrm{A}_{1}\right)$ seems likely, though not conclusive. From the calculations and the presence of a

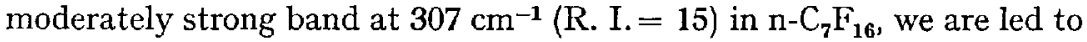
assign $\gamma_{t}\left(\mathrm{CF}_{2}\right)\left(\mathrm{A}_{1}\right)$ to this frequency. The computations of section III. A. 2. indicate that a planar zig-zag $-\left(\mathrm{CF}_{2 / n}\right.$ chain would have skeletal modes in the vicinity of $570 \mathrm{~cm}^{-1}$. The only Raman band in this region in $n-C_{7} F_{18}$ is at $607 \mathrm{~cm}^{-1}(R . I .=2)$, and we therefore assign it to the $A_{1}$ skeletal vibration in PTFE. On the basis of this calculation the $\mathrm{E}_{1}$ skeletal mode is not identifiable. (Although a possible candidate is the $\sigma$ band at $553 \mathrm{~cm}^{-1}$, it seems more likely at present that this band is to be assigned to the $\delta\left(\mathrm{CF}_{2}\right)\left(\mathrm{E}_{1}\right)$ mode.) The remaining $\mathrm{E}_{1}$ modes have been assigned on the basis of the calculations for the hypothetical zig-zag chain [LIANG and KRIMM (111)], and seem to lead to a satisfactory interpretation of combination bands. It would be desirable to have polarization data on some of these bands in order to be more certain of the assignment. The 


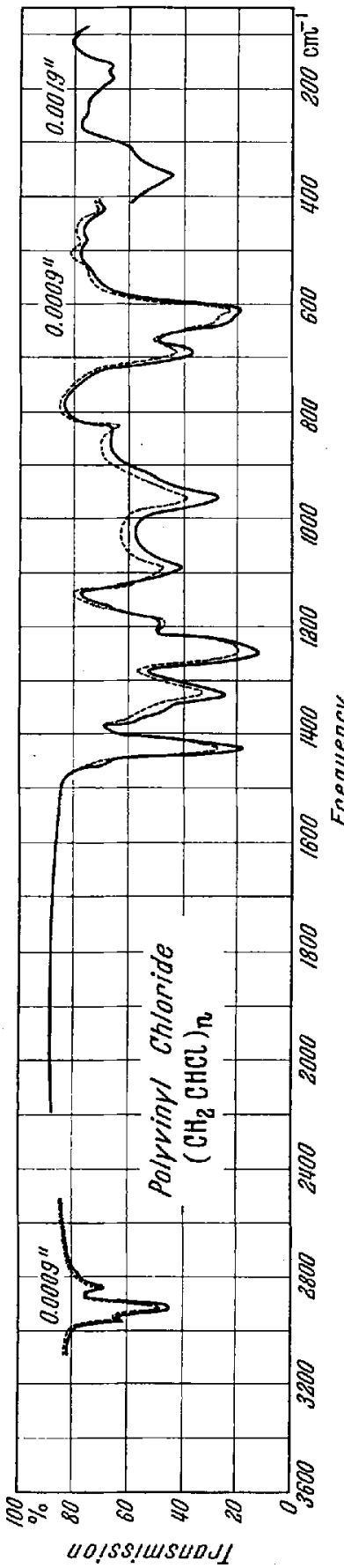

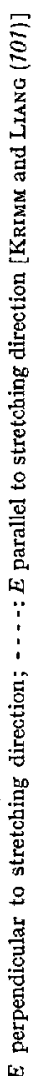

low frequency bands, and the modes associated with the amorphous bands, remain to be satisfactorily interpreted.

While a fairly satisfactory interpretation has been achieved for the spectrum of PTFE, it is evident that several factors need additional clarification in order to make the assignments firmer. A Raman spectrum of PTFE is required. The polarization of the $1210 \mathrm{~cm}^{-1}$ band and several of the conbination bands needs more careful determination. The computations for the planar zig-zag chain may be worth redoing. And it would be highly desirable to obtain spectra of crystalline fluorocarbons, analogous to the $n$-paraffins, for a more detailed analysis of certain aspects of the spectrum. It might be noted that the present analysis of the spectrum of PTFE serves as a satisfactory basis for a general understanding of the polychlorotrifluoroethylene spectrum [LIANG and KRIMM (171)].

\section{Polyvinyl Chloride}

Polyvinyl chloride (PVC), $-\mathrm{CH}_{2} \mathrm{CHCl}_{\bar{n}}$, is chemically representative of a large class of polymers, the vinyl polymers, in which a single hydrogen atom on alternate carbon atoms is replaced by another atom or a group of atoms. These polymers present an interesting structural problem since, depending on the position of the substituent, different configurational isomers can exist. As a result of the discovery and development of stereospecific catalysts [NATTA (150)], it has been possible to synthesize almost completely isotactic and syndiotactic polymers. (In the former all of the substituents are on the same side of the plane of the zig-zag carbon backbone, while in the latter the substituents alternate on either side of this plane.) 
Table 11. Infraved Spectrum of Polyvinyi Chloride

\begin{tabular}{|c|c|c|c|c|}
\hline$\underset{\mathrm{Cm}^{-1}}{\text { Frequency, }}$ & R. I. & Pol'n & Phase & Assignment \\
\hline 102 & $v w$ & & & $\mathrm{R}_{\mathrm{o}}^{\prime}\left(\mathrm{B}_{1 u}\right)$ \\
\hline 160 & $\mathrm{w}$ & & & $v\left(\frac{\pi}{2}\right)\left(a_{1}\right)_{0}\left(B_{2 u}\right)$ \\
\hline 182 & w & & & $v\left(\frac{\pi}{2}\right)\left(b_{2}\right)_{s}\left(B_{3 u}\right)$ \\
\hline 315 & vw & & & $\gamma_{s 0}(\mathrm{CCl})_{0 i}\left(\mathrm{~B}_{3 w}\right)$ \\
\hline 363 & $w$ & & & $\delta(\mathrm{CCl})_{i 0}\left(\mathrm{~B}_{2 u}\right)$ \\
\hline 430 & w & $\sigma$ & & $\delta(\mathrm{CCl})_{0 \gamma}\left(\mathrm{B}_{14}\right)$ \\
\hline 487 & $w$ & $\sigma$ & & $v_{-}\left(\frac{\pi}{2}\right)_{0}\left(B_{1 u}\right)$ \\
\hline 540 & $\mathrm{w}$ & $\pi ?$ & & $\delta(\mathrm{CCl})\left(\mathrm{B}_{2 u}\right)+v\left(\frac{\pi}{2}\right)\left(\mathrm{B}_{1 g}\right)=545\left(\mathrm{~B}_{3 u}\right) ?$ \\
\hline 604 & $\mathbf{s}$ & $\sigma$ & C & $v(\mathrm{CCl})_{10}\left(\mathrm{~B}_{2 u}\right)$ \\
\hline 615 & s & $\sigma$ & A & $\nu(\mathrm{CCl})(\mathrm{Cl}$ trans to $\mathrm{H})$ \\
\hline 642 & $\mathrm{~s}$ & $\sigma$ & $\mathrm{c}$ & $v(\mathrm{CCl})_{01}\left(\mathrm{~B}_{1 u}\right)$ \\
\hline 693 & $\mathrm{~m}$ & $\sigma$ & A & $y(\mathrm{CCl})(\mathrm{Cl}$ trans to $\mathrm{C})$ \\
\hline 764 & $\mathrm{vw}$ & $\pi ?$ & & $v(\mathrm{CCl})\left(\mathrm{B}_{2 u}\right)+v\left(\frac{\pi}{2}\right)\left(\mathrm{B}_{1 \theta}\right)=786\left(\mathrm{~B}_{3 t}\right) ?$ \\
\hline 833 & $w$ & $\pi$ & & $\gamma_{r}\left(\mathrm{CH}_{2}\right)_{0 i}\left(\mathrm{~B}_{3 u}\right)$ \\
\hline$\sim 920$ & $\mathrm{vw}$ & & & $v_{+}\left(\frac{3 \pi}{2}\right)\left\langle\mathrm{B}_{\mathrm{I} u}\right) ?$ \\
\hline 960 & $\mathrm{~m}$ & $\sigma$ & & $\gamma_{t}\left(\mathrm{CH}_{2}\right)_{i t}\left(\mathrm{~B}_{1+u}\right)$ \\
\hline 1095 & $\mathrm{~m}$ & $\sigma$ & & $y_{+}(0)_{0}\left(\mathrm{~B}_{3 u}\right)$ \\
\hline 1125 & $\mathrm{w}$ & $\pi$ & $C$ ? & $y_{+}(\pi)_{\gamma}\left(B_{3 u}\right)$ \\
\hline 1167 & $v w$ & & & $\gamma_{l}\left(\mathrm{CH}_{2}\right)_{00}\left(\mathrm{~B}_{2 u}\right) ?$ \\
\hline 1197 & $\mathrm{w}$ & $\pi$ & & $\begin{array}{l}\gamma_{r}\left(\mathrm{CH}_{2}\right)_{o r}\left(\mathrm{~B}_{3 u}\right)+\delta(\mathrm{CCl})_{r}\left(\mathrm{~A}_{q}\right)(\sim 363) \\
\quad=1196\left(\mathrm{~B}_{3 u}\right) ?\end{array}$ \\
\hline 1230 & vw & $\pi$ & C? & $\gamma_{p o}(\mathrm{CH})_{0 r}\left(\mathrm{~B}_{3 u}\right)$ \\
\hline$\sim 1240$ & $\mathrm{w}-\mathrm{mis}$ & & A ? & $2 \times 615=1230$ \\
\hline 1254 & $\mathrm{~s}$ & $\sigma$ & & $\delta(\hat{\mathrm{CH}})_{t 0}\left(\mathrm{~B}_{24}\right)$ \\
\hline 1333 & $\mathrm{~m}$ & $\sigma$ & & $\delta(\mathrm{CH})_{n i}\left(\mathrm{~B}_{1 u}\right)$ \\
\hline 1352 & $\mathrm{w}$ & $\sigma$ & & $\delta(\mathrm{CH})\left(\mathrm{A}_{q}\right)+\mathrm{R}_{0}^{\prime}\left(\mathrm{B}_{1 u}\right)=1356\left(\mathrm{~B}_{1 u}\right)$ \\
\hline 1380 & $w$ & $\pi$ & & $\gamma_{p p}\left(\mathrm{CH}_{2}\right)_{i q}\left(\mathrm{~B}_{3 u}\right)$ \\
\hline 1424 & $\mathrm{~s}$ & $\sigma$ & $\mathrm{C}$ & $\delta\left(\mathrm{CH}_{2}\right)_{v o}\left(\mathrm{~B}_{2 u}\right)$ \\
\hline 1431 & $\mathrm{~s}$ & $\sigma$ & A & $\delta\left(\mathrm{CH}_{2}\right.$ \\
\hline 2820 & $\mathrm{w}$ & $\sigma$ & & $\nu(\mathrm{CH})_{10}\left(\mathrm{~B}_{20}\right)$ \\
\hline 2849 & $\mathbf{w}$ & $\sigma$ & & $v_{s}\left(\mathrm{CH}_{2}\right)_{60}\left(\mathrm{~B}_{2 u}\right)$ \\
\hline 2914 & $\mathrm{~m}$ & $\sigma$ & & $\nu_{u}\left(\mathrm{CH}_{2}\right)_{i i}\left(\mathrm{~B}_{1 w}\right)$ \\
\hline 2930 & $\mathbf{w}$ & $\pi$ ? & & $v_{a}\left(\mathrm{CH}_{2}\right)_{0 s}\left(\mathrm{~B}_{2 u}\right)$ \\
\hline 2968 & $\mathrm{mw}$ & $\sigma$ & & $v(\mathrm{CH})_{0}\left(\mathrm{~B}_{1 \mu}\right)$ \\
\hline
\end{tabular}

Polyvinyl chloride furnishes a good example of how the infrared spectrum can provide confirmatory evidence on the nature of the isomeric structure.

Chemical evidence [MARvel, SAMPLE, and Roy (126)] as well as recent infrared studies [HoDGKINS (78)] have established that PVC has mainly a head-to-tail chemical structure, i. e., - $-\mathrm{CH}_{2} \mathrm{CHClCH}_{2} \mathrm{CHClCH}_{2} \mathrm{CHCl}---$. Early $x$-ray work [FULLer (64)] had indicated that the chain configuration might be syndiotactic, but since this depended primarily on interpretation of the $5.1 \mathrm{~A}$ fiber axis repeat, the remainder of the pattern not being detailed enough to test this hypothesis adequately, this structure could not be considered to be firmly established. A detailed analysis 
of the infrared spectrum of PVC [KRIMM and LIANG (101)] provided additional support for a syndiotactic structure, and this has been strengthened by recent studies of more highly ordered polymers [KRIMM, Berens, Folt, and Shipman $(105,106)]$. A more detailed x-ray diffrac-

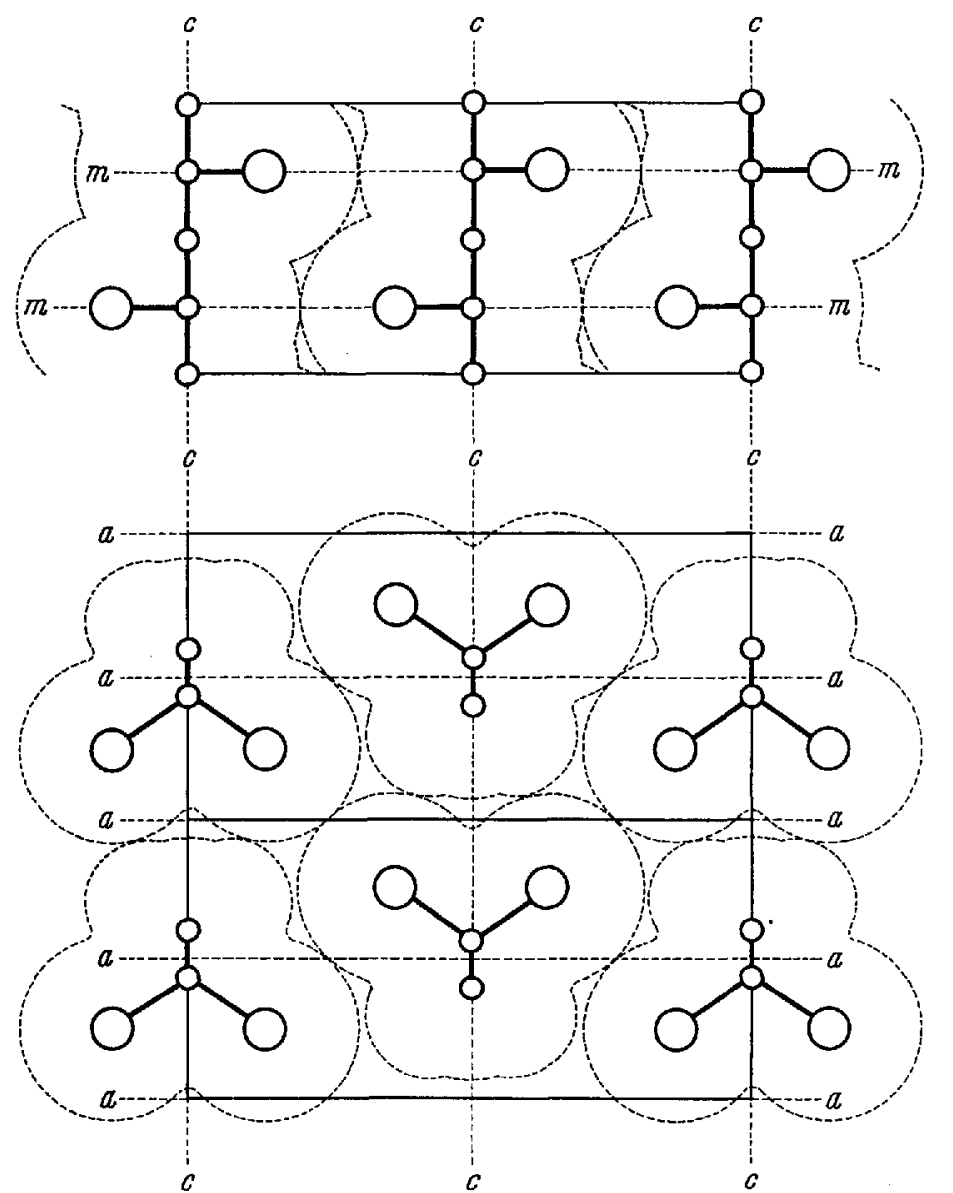

Fig. 10. Structure and symmetry elements of crystalline polyvinyl chloride [NATTA and CorraptN1 (754 $\}$

tion study of PVC [NATTA and CoRRadini (154)] confirms these conclusions as to the configuration of the chain. A careful study of the spectrum of PVC should therefore provide a guide to the identification and study of other syndiotactic polymers.

The spectrum of an oriented specimen of PVC is shown in Fig. 9. This specimen is a typical low-crystallinity PVC. Higher crystallinity specimens, prepared by polymerization at low temperatures [GRISENTHwaite and Hunter (68); Krimm, Berens, Folt, and Shipman (105); 
Shimanouchi, Tsuchiya, and Mizushima (196)] and by irradiation of urea-canal complexes [Krimm, Berens, Folt, and Shipman (106); Shimanouchi, Tsuchiya, and Mizushima $(196,197)]$, have also been studied. Band positions are given in Table 11, together with polarization data [KRIMM and LIANG (101); NARITA, ICHINOHE, and EnoMoto (144); Shimanovchi, Tsuchiya, and Mrzushima (196)] and data on the

Table 12. Results of Symmetry Analysis for Crystalline Polyvïnyl Chloride

\begin{tabular}{|c|c|c|c|c|c|c|}
\hline \multicolumn{2}{|c|}{ Species and activity } & \multicolumn{5}{|c|}{ Symmetry modes } \\
\hline$A_{g}$ & $\mathrm{R}$ & $\begin{array}{l}v(\mathrm{CH})_{i i} \\
v(\mathrm{CCl})_{i i}\end{array}$ & $\begin{array}{l}\delta(\mathrm{CH})_{i i} \\
\delta(\mathrm{CCI})_{i i}\end{array}$ & $\begin{array}{l}v_{i}\left(\mathrm{CH}_{2}\right)_{i j} \\
v_{+}(0)_{i}\end{array}$ & $\begin{array}{l}\delta\left(\mathrm{CH}_{2}\right)_{i i} \\
v\left(\frac{\pi}{2}\right)\left(\mathrm{a}_{1}\right)_{i}\end{array}$ & $\begin{array}{c}\gamma_{1}\left(\mathrm{CH}_{2}\right)_{0 i} \\
\mathrm{~T}_{b}^{\prime}\end{array}$ \\
\hline $\mathrm{B}_{10}$ & $\mathrm{R}$ & $\begin{array}{l}\gamma_{w}(\mathrm{CH})_{00} \\
\nu_{+}(\pi)_{0}\end{array}$ & $\begin{array}{l}y_{a}\left(\mathrm{CH}_{2}\right)_{00} \\
v\left(\frac{\pi}{2}\right)\left(b_{2}\right)_{0}\end{array}$ & $\begin{array}{c}\gamma_{w}\left(\mathrm{CH}_{2}\right)_{i 0} \\
\mathrm{~T}_{0}^{\prime}\end{array}$ & $\gamma_{r}\left(\mathrm{CH}_{2}\right)_{00}$ & $\gamma_{\omega}(\mathrm{CCl})_{00}$ \\
\hline $\mathrm{B}_{2 \sigma}$ & $\mathrm{R}$ & $\begin{array}{l}\gamma_{w}(\mathrm{CH})_{i i} \\
\nu_{+}\left(\frac{\pi}{2}\right)_{0}\end{array}$ & $\begin{array}{l}v_{*}\left(\mathrm{CH}_{2}\right)_{0 i} \\
v_{-}\left(\frac{3 \pi}{2}\right)_{0}\end{array}$ & $\delta\left(\mathrm{CH}_{2}\right)_{0 r}$ & $\gamma_{\gamma}\left(\mathrm{CH}_{2}\right)_{i \prime}$ & $\gamma_{w}(\mathrm{CCl})_{d i}$ \\
\hline$B_{3 p}$ & $\mathrm{R}$ & $\begin{array}{l}\nu(\mathrm{CH})_{00} \\
\nu(\mathrm{CCl})_{00}\end{array}$ & $\begin{array}{l}\delta(\mathrm{CH})_{0} 0 \\
\delta(\mathrm{CCl})_{00}\end{array}$ & $\begin{array}{l}v_{a}\left(\mathrm{CH}_{2}\right)_{i 0} \\
v_{-}\left(\frac{\pi}{2}\right)_{i}\end{array}$ & $\begin{array}{l}\gamma_{w}\left(\mathrm{CH}_{2}\right)_{00} \\
v_{+}\left(\frac{3 \pi}{2}\right)_{1}\end{array}$ & $\begin{array}{c}\gamma_{r}\left(\mathrm{CH}_{2}\right)_{\gamma_{0}} \\
\mathrm{R}_{i}^{\prime} \mathrm{T}_{a}^{\prime}\end{array}$ \\
\hline $\mathrm{A}_{u}$ & - & $\begin{array}{l}\gamma_{20}(\mathrm{CH})_{10} \\
\nu_{+}\left(\frac{\pi}{2}\right)_{i}\end{array}$ & $\begin{array}{l}v_{4}\left(\mathrm{CH}_{2}\right)_{00} \\
v_{-}\left(\frac{3 \pi}{2}\right)_{i}\end{array}$ & $\delta\left(\mathrm{CH}_{2}\right)_{00}$ & $\gamma_{1}\left(\mathrm{CH}_{2}\right)_{i 0}$ & $\gamma_{\infty}(\mathrm{CCl})_{i_{0}}$ \\
\hline $\mathrm{B}_{1 u}$ & $\mathrm{IR}, \sigma_{a}$ & $\begin{array}{l}\nu(\mathrm{CH})_{0} t \\
\nu(\mathrm{CCl})_{0 i}\end{array}$ & $\begin{array}{l}\delta(\mathrm{CH})_{0 i} \\
\delta(\mathrm{CCl})_{0 i}\end{array}$ & $\begin{array}{l}v_{a}\left(\mathrm{CH}_{2}\right)_{i i} \\
v_{-}\left(\frac{\pi}{2}\right)_{0}\end{array}$ & $\begin{array}{l}\gamma_{w}\left(\mathrm{CH}_{2}\right)_{0 i} \\
y_{+}\left(\frac{3 \pi}{2}\right)_{0}\end{array}$ & $\begin{array}{c}\gamma_{r}\left(\mathrm{CH}_{2}\right)_{i i} \\
\mathrm{R}_{0}^{\prime} \mathrm{T}_{a}\end{array}$ \\
\hline $\mathrm{B}_{2 u}$ & $\operatorname{IR}, \sigma_{b}$ & $\begin{array}{l}v(\mathrm{CH})_{i 0} \\
v(\mathrm{CCl})_{i 0}\end{array}$ & $\begin{array}{l}\delta(\mathrm{CH})_{10} \\
\delta(\mathrm{CCl})_{10}\end{array}$ & $\begin{array}{l}v_{1}\left(\mathrm{CH}_{2}\right)_{i 0} \\
v_{+}(0)_{0}\end{array}$ & $\begin{array}{l}\delta\left(\mathrm{CH}_{2}\right)_{i_{0}} \\
v\left(\frac{\pi}{2}\right)\left(\mathrm{a}_{1}\right)_{0}\end{array}$ & $\begin{array}{c}\gamma_{l}\left(\mathrm{CH}_{2}\right)_{00} \\
\mathrm{~T}_{b}\end{array}$ \\
\hline$B_{i u}$ & IR, $\pi$ & $\begin{array}{l}\gamma_{w}(\mathrm{CH})_{0 i} \\
\nu_{+}(\pi)_{i}\end{array}$ & $\begin{array}{l}v_{a}\left(\mathrm{CH}_{2}\right)_{0 i} \\
v\left(\frac{\pi}{2}\right)\left(\mathrm{b}_{2}\right)_{i}\end{array}$ & $\begin{array}{c}\gamma_{v}\left(\mathrm{CH}_{2}\right)_{i i} \\
\mathrm{~T}_{0}\end{array}$ & $\gamma_{r}\left(\mathrm{CH}_{2}\right)_{0 i}$ & $\gamma_{* 0}(\mathrm{CCl})_{01}$ \\
\hline
\end{tabular}

crystalline or amorphous origin of some of the bands. There does not seem to be any good evidence for the presence of six bands in the $3 \mu$ region, as has been suggested [Ambrose, Elliott, and Temple (6)]. Raman spectra of PVC or of appropriate low molecular weight analogue molecules are not available.

On the basis of $x$-ray and infrared studies we may now accept the syndiotactic chain configuration for crystalline PVC. If, in addition, we accept the proposed crystal structure [NATTA and CoRRADINI (154)], it is possible to base the symmetry analysis of the spectrum on the unit cell instead of just on a single chain, as was done in the earlier work [KRIMM and LIANG (101)]. The results of this analysis are given here. The unit cell, shown in Fig. 10, has the following symmetry elements: $E, C_{2}^{s}(a)$, $C(b), C_{2}^{s}(c), i, \sigma_{g}(b c), \sigma_{o}(a c)$, and $\sigma(a b)$. These form a group isomorphous 
with $D_{2 h}$, and by applying the methods of section III. A. 1. we can determine the number of normal modes and their activity in any given symmetry species. It is further possible to describe the symmetry modes of the unit cell in terms of modes of a single chain. The results of this analysis are listed in Table 12. The subscripts refer to in-phase (i) and out-of-phase (o) motions, the first subscript referring to the nature of the mode in a single chain and the second subscript to the relationship between the motions of the two chains in the unit cell. The $T^{\prime}$ and $R^{\prime}$ modes refer to translatory and rotatory motions of the chain as a unit. In the former modes the chains move against each other along the axis indicated; in the latter modes the chains librate about their axes, in one case $\left(R_{i}^{\prime}\right)$ both rotating clockwise at the same time, and in the other $\left(R_{0}^{\prime}\right)$ one rotating clockwise while the other rotates counter-clockwise. As far as the infrared spectrum is concerned, the analysis according to the unit cell yields only one more active mode, viz., $R_{0}^{\prime}$, than was predicted by the single chain analysis. In the Raman spectrum four new modes become active, $T_{a}^{\prime}, T_{t}^{\prime}, T_{c}^{\prime}$, and $R_{i}^{\prime}$. It should be noted that infrared active bands are now Raman inactive, and vice versa. Actually the frequencies of most of the Raman fundamentals should be quite close to the corresponding infrared fundamentals; they will be separated only as a result of interaction between the chains in the unit cell.

In arriving at a satisfactory analysis of the spectrum we must make use not only of the polarization data, but also of the results of deuteration studies, full [NARITA, ICHINOHE, and ENomoto (145)] and partial [FolT, ShIPMAN, and Berens (55)], and studies of $\mathrm{C}-\mathrm{Cl}$ frequencies in small molecules, [Mizushima, Shimanouchi, Nakamura, Hayashi, and Tsuchiya (139); Shimanouchi, Tsuchiya, and Mizushima (196)]. The lack of the Raman spectrum is a definite handicap, but is in part mitigated by the expectation that many of the Raman active fundamentals should be close to the frequencies of infrared active fundamentals.

The above-mentioned studies on $\mathrm{C}-\mathrm{Cl}$ frequencies of small molecules lead to a fairly unambiguous assignment of the $v(\mathrm{CCl})$ modes of PVC. These studies show that the $v(\mathrm{CCl})$ modes occur in fairly restricted frequency ranges depending on whether the $\mathrm{Cl}$ atom is trans to a $\mathrm{H}$ atom or to a $\mathrm{C}$ atom across the common $\mathrm{C}-\mathrm{C}$ bond. For secondary chlorides, the former modes are found at $605-650 \mathrm{~cm}^{-1}$ and the latter modes at $670-700 \mathrm{~cm}^{-1}$. The absorption in the $600-700 \mathrm{~cm}^{-1}$ region of PVC is complex, but studies on highly crystalline samples made by polymerization in a urea-canal complex [KrIMm, Berens, Folt, and Shipman (106)] indicate that only two bands, at 604 and $642 \mathrm{~cm}^{-1}$, are to be associated with the crystalline phase. These both correspond to $v(\mathrm{CCl})$ with $\mathrm{Cl}$ trans to $\mathrm{H}$, and are both $\sigma$, in agreement with expectations. Tilting experiments with partially double oriented specimens indicate that the $604 \mathrm{~cm}^{-1}$ band 
is polarized along the $b$-axis of the crystal while the $642 \mathrm{~cm}^{-1}$ band is polarized along the $a$-axis. This suggests the particular assignment of the $\mathrm{B}_{1 u}$ and $\mathrm{B}_{2 u} \nu(\mathrm{CCl})$ modes shown in Table 11. It is interesting to note that full deuteration of the chain shifts these $v(\mathrm{CCl})$ modes from a mean position at $623 \mathrm{~cm}^{-1}$ to one at about $581 \mathrm{~cm}^{-1}$ [NARITA, ICHINOHE, and ENoмOTo (145)]. This points up the fact that what we call a $v(\mathrm{CCl})$ mode is actually a vibration which also significantly involves the carbon chain skeleton. It is therefore not unreasonable to expect that the interaction between neighboring $\mathrm{Cl}$ atoms in the syndiotactic repeat unit will be large enough to give the observed $38 \mathrm{~cm}^{-1}$ separation between $\nu(\mathrm{CCI})_{i}$ and $v(\mathrm{CCl})_{0}$, even though at first glance the large separation between the $\mathrm{Cl}$ atoms might tend to indicate a weak interaction between their stretching modes. This is an example of a case in which simple group frequency concepts are not completely adequate. The remaining bands in this region, at about 615 and $693 \mathrm{~cm}^{-1}$, undoubtedly arise from chains in the amorphous regions which are atactic, so that the splitting disappears, and/or in non planar zig-zag conformations which place $\mathrm{Cl}$ trans to $\mathrm{H}$ at some points and trans to $\mathrm{C}$ at others along the chain. The disappearance of the $604,642 \mathrm{~cm}^{-1}$ doublet and its replacement by a single broad band at about $615 \mathrm{~cm}^{-1}$ when PVC is swollen in cyclohexanone or heated [Shimanouchi, Tsuchiya, and Mizushima (196)] support this conclusion. There does not seem to be any good evidence for associating any of these bands with a helical isotactic structure, as has been proposed [Shimanouchi, TsuchiYa, and Mizushima (197)].

The $v(\mathrm{CH})$ and $\nu\left(\mathrm{CH}_{2}\right)$ modes can be assigned with fair confidence on the basis of studies on partially deuterated PVC's [FoLT, SHIPMAN, and BerEns (55)]. These confirm the assignments previously made [KRIMM and LIANG (101)]. For example, the $2968 \mathrm{~cm}^{-1}$ band disappears only when $\mathrm{CDCl}$ replaces $\mathrm{CHCl}$, thus identifying this as a $v(\mathrm{CH})$ mode of $\mathrm{PVC}$. Tilting experiments on a partially double oriented specimen favor its assignment to $\nu(\mathrm{CH})_{0}$. The presence of a weak band at about $2930 \mathrm{~cm}^{-1}$ which seems to have $\pi$ dichroism provides a likely assignment for the $v_{a}\left(\mathrm{CH}_{2}\right)_{0 i}\left(\mathrm{~B}_{3 u}\right)$ mode predicted by the symmetry analysis. The $2914 \mathrm{~cm}^{-1}$ band is reasonably assigned to the $\sigma v_{a}\left(\mathrm{CH}_{2}\right)$ mode. The 2849 and $2820 \mathrm{~cm}^{-1}$ bands are then assigned to the $v_{s}\left(\mathrm{CH}_{2}\right)$ and $v(\mathrm{CH})_{i}$ modes.

We consider next the remaining $\mathrm{B}_{3 u}$ modes which are to be assigned. Of these, the $\gamma_{w}(\mathrm{CCl})_{0 i}$ and $v\left(\frac{\pi}{2}\right)\left(b_{2}\right)_{i}$ are expected to be found below $600 \mathrm{~cm}^{-1}$, while the four others should occur roughly in the $600-1400$ $\mathrm{cm}^{-1}$ region. There are five $\pi$ bands observed in this region; for the weak band at $764 \mathrm{~cm}^{-1}$ the $\pi$ dichroism is uncertain. Studies on partially deuterated PVC's [Folt, Shipman, and Berens (55)] show that the $833 \mathrm{~cm}^{-1}$ band moves progressively down until it is at $670 \mathrm{~cm}^{-1}$ in the 
fully deuterated polymer, making this most likely the $\gamma_{r}\left(\mathrm{CH}_{2}\right)_{0 i}$ mode. Its position, as predicted from calculations (see section III. A. 2.), and relatively small shift on deuteration suggest that the $1125 \mathrm{~cm}^{-1}$ band is to be assigned to $v_{+}(\pi)_{i}$. The remaining assignments are less certain, but 1230: $\gamma_{w}(\mathrm{CH})_{0 i}$ and 1380: $\gamma_{w}\left(\mathrm{CH}_{2}\right)_{i i}$ are favored. It might be noted that the $1230 \mathrm{~cm}^{-1}$ band cannot be an overtone or combination involving $v(\mathrm{CCl})$ modes since these cannot combine in any way to give a $\mathrm{B}_{3 u}$ mode. In accordance with earlier suggestions [KRIMm and LIANG (101)], the $\gamma_{w}(\mathrm{CCl})_{0 i}$ and $\nu\left(\frac{\pi}{2}\right)\left(b_{2}\right)_{i}$ modes are most reasonably assigned to bands at $315 \mathrm{~cm}^{-1}$ and $182 \mathrm{~cm}^{-1}$ respectively.

The remaining $\mathrm{B}_{1 u}$ and $\mathrm{B}_{2 u}$ modes can be assigned with fair certainty on the basis of deuteration and other data. The $1424 \mathrm{~cm}^{-1}$ band is undoubtedly $\delta\left(\mathrm{CH}_{2}\right)_{i 0}\left(\mathrm{~B}_{2 u}\right)$; it shifts to $1110 \mathrm{~cm}^{-1}$ in the fully deuterated polymer. The $1431 \mathrm{~cm}^{-1}$ band, which is strongest in the least crystalline polymers, is probably also a $\delta\left(\mathrm{CH}_{2}\right)$ mode. The frequency shift may indicate a different environment for the $\mathrm{CH}_{2}$ groups giving rise to this mode, e.g., their presence between neighboring $\mathrm{Cl}$ atoms which are on the same side of the carbon zig-zag, as in atactic portions of molecules. The 1333 and $1254 \mathrm{~cm}^{-1}$ bands are probably the two $\delta(\mathrm{CH})$ modes expected in the syndiotactic unit of structure. This is supported by their shift to $1018 \mathrm{~cm}^{-1}$ and $960 \mathrm{~cm}^{-1}$ respectively in the fully deuterated polymer [NARITA, ICHNOHE, and ENOMOTO (145)], and by the disappearance of these two bands and their replacement by a single band midway between (at $\sim 1290 \mathrm{~cm}^{-1}$ ) in chlorinated PVC [FUCHs and Louis (63)]. The latter is just what would be expected from chlorination of the $\mathrm{CH}_{2}$ groups to $\mathrm{CHCl}$, which would destroy the syndiotactic configuration of the original polymer. The specific assignments to symmetry species are suggested by tilting experiments on partially double oriented specimens. The $1095 \mathrm{~cm}^{-1}$ band appears to shift to $1040 \mathrm{~cm}^{-1}$ on deuteration. Its position and small shift suggest its assignment to $\boldsymbol{v}_{+}(0)_{0}$. The $960 \mathrm{~cm}^{-1}$ band moves progressively, with increasing deuteration [Folt, Shipman, and Berens (55)] to $787 \mathrm{~cm}^{-1}$ in the fully deuterated polymer. This shift suggests its assignment to $\gamma_{r}\left(\mathrm{CH}_{2}\right)_{i i}$. It will be noted that the isotopic frequency shifts observed here bear little relation to the values predicted by the treatment of section III. B. 2 . This is not unexpected, since that treatment assumed an isolated $\mathrm{CH}_{2}$ (or $\mathrm{CH}$ ) group whereas we know that some of the modes in PVC are most unlikely to be significantly confined to a single group. On the other hand, known skeletal modes shift by about 1.05-1.07 on complete deuteration, and it is therefore unlikely that the shift of the $960 \mathrm{~cm}^{-1}$ band to $787 \mathrm{~cm}^{-1}$ (ratio $=1.22$ ) could be associated with a skeletal vibration. The assignments of the other bands expected in this general region, viz., $\boldsymbol{v}_{+}\left(\frac{s \pi}{2}\right)_{0}$ 
and $\gamma_{t}\left(\mathrm{CH}_{2}\right)_{00}$, cannot be made with any great certainty, but calculations for the former mode and the polyethylene assignments for the latter suggest the bands at $920 \mathrm{~cm}^{-1}$ and $1167 \mathrm{~cm}^{-1}$ respectively as possibilities. For the remaining $\mathrm{Cl}$ and skeletal modes we follow earlier arguments [KRIMM and LIANG (101)] based on study of small molecules and calculations. The $R_{0}^{\prime}\left(\mathrm{B}_{1 u}\right)$ mode may possibly be assigned to the band at $102 \mathrm{~cm}^{-1}$. The remaining weak bands are probably combination bands, but it is difficult to be certain because of the lack of a Raman spectrum.

It will be seen that the overall set of proposed assignments for $\mathrm{PVC}$ is quite satisfactory, although uncertainties do exist on some points. This provides important evidence in support of a syndiotactic chain configuration and of the crystal structure which has been proposed. It will be noted that the characteristic spectral feature of a syndiotactic structure is the doubling in some of the bands, and this should provide a significant guide in the analysis of other syndiotactic polymers. Several assignments, as we have noted, require additional confirmation. This could be achieved by extended studies on partially deuterated PVC's, a polarized spectral study of oriented highly crystalline urea complex polymers, and further work on better double oriented specimens.

\section{Polyvinyl Alcohol}

The spectrum of polyvinyl alcohol (PVA), $-\left(\mathrm{CH}_{2} \mathrm{CHOH}_{\bar{n}}\right.$, was the subject of much early study [THOMPson and ToRkington (228)]; Elliott, Ambrose, and Temple (53); Blout and Karplus (17); Ambrose, Elliott, and Temple (6); Glatt, Webber, Seaman, and Ellis (66); GLATT and ELIIS (65)], but with only

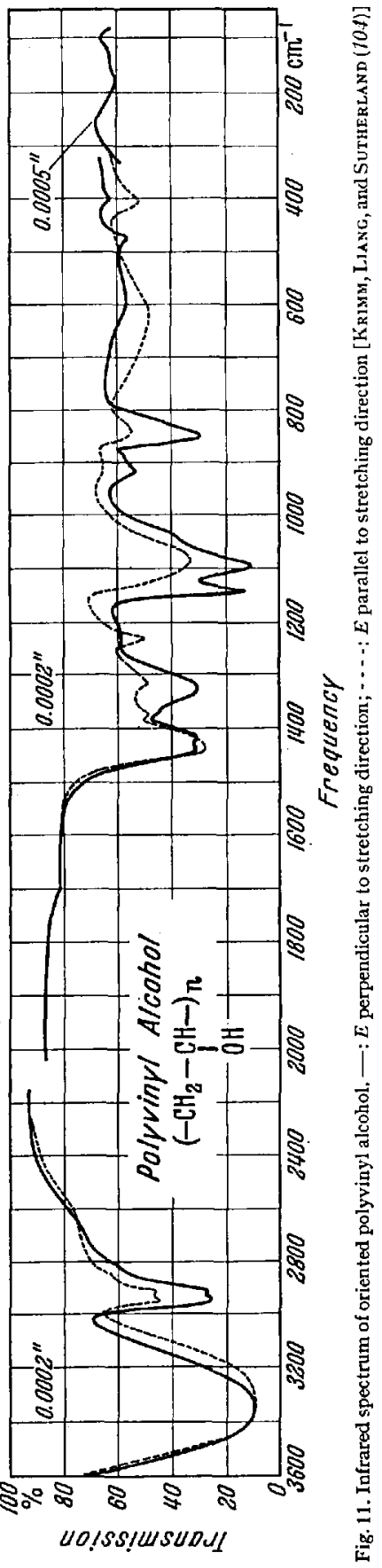


Table 13. Infrared Spectrum of Polyvinyl Alcohol

\begin{tabular}{|c|c|c|c|}
\hline $\begin{array}{l}\text { Frequency, } \\
\mathrm{cm}^{-1}\end{array}$ & R.I. & Pol'n & Assignment \\
\hline 135 & vvw & & \\
\hline 185 & $\mathrm{vw}$ & & $v(\mathrm{OH} \ldots \mathrm{O})$ \\
\hline 360 & vvw & $\sigma$ & $2 \times v(\mathrm{OH} \ldots \mathrm{O})=370$ \\
\hline 410 & $\mathrm{w}$ & $\pi$ & $\gamma_{w}(\mathrm{CO})$ \\
\hline 480 & $\mathrm{w}$ & $\sigma$ & $\delta(\mathrm{CO})$ \\
\hline $\begin{array}{l}610 \\
630\end{array}$ & $\mathrm{w}$ & $\sigma\}$ & $\gamma_{w}(\mathrm{OH})$ \\
\hline $\begin{array}{l}630 \\
825\end{array}$ & $\begin{array}{l}\mathrm{m} \\
\mathrm{vw}\end{array}$ & $\begin{array}{l}\pi 1 \\
\sigma\end{array}$ & $\gamma_{r}\left(\mathrm{CH}_{2}\right)$ \\
\hline 850 & m & $\sigma_{+}^{\sigma_{+}}$ & $\begin{array}{l}\gamma_{\mathrm{r}}\left(\mathrm{CH}_{2}\right) \\
\gamma_{r}\left(\mathrm{CH}_{2}\right) \text { (amorphous) }\end{array}$ \\
\hline 890 & vw & $\sigma_{11}$ & $\delta(\mathrm{CO})+\gamma_{w}(\mathrm{CO})=890$ \\
\hline 915 & $w$ & $\sigma_{11}$ & $\begin{array}{l}v_{+}(0) \\
(v(\mathrm{CC})(\mathrm{C}-\mathrm{C}-\mathrm{C}-\mathrm{C} \text { unit }) ?\end{array}$ \\
\hline 1040 & vw & $\sigma_{\perp}$ & $\left\{\begin{array}{lll}1 & 1 \\
0 & 0\end{array}\right.$ \\
\hline 1085 & vw & $\pi(?)$ & $\underset{\gamma_{+}(\pi)}{\gamma_{v}(\mathrm{CO})}+\gamma_{w}(\mathrm{OH})=1040$ \\
\hline 1096 & $s$ & $\begin{array}{c}\pi(t) \\
\sigma\end{array}$ & $v(C O)$ \\
\hline 1141 & $\mathrm{~m}$ & $\sigma_{11}(\dot{\mathbb{C}})$ & 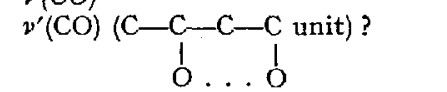 \\
\hline 1215 & vw & $\sigma$ & $2 \times 610=1220 ?$ \\
\hline 1235 & $\mathbf{w}$ & $\pi$ & $\gamma_{v e}(\mathrm{CH})$ \\
\hline $\left.\begin{array}{l}1320 \\
1326\end{array}\right\}$ & s & $\sigma$ & $\delta(\mathrm{CH}+\mathrm{OH})$ \\
\hline $\begin{array}{l}1320) \\
1376\end{array}$ & $\mathrm{w}$ & ? & $\delta(\mathrm{CH}+\mathrm{OH})$ \\
\hline 1430 & s & $\sigma$ & $\delta\left(\mathrm{CH}_{2}\right)$ \\
\hline 1446 & s & $\pi$ & $\gamma_{\nu}\left(\mathrm{CH}_{2}\right)$ \\
\hline$\sim 2590$ & $\mathrm{vw}$ & $\pi ?$ & $\gamma_{t v}\left(\mathrm{CH}_{2}\right)+v^{\prime}(\mathrm{CO})=2587(?)$ \\
\hline$\sim 2660$ & $\mathrm{vw}$ & $\sigma$ & $\delta\left(\mathrm{CH}_{2}\right)+\gamma_{v o}(\mathrm{CH})=2665$ \\
\hline 2840 & vw & $\sigma$ & $v(\mathrm{CH})$ \\
\hline 2910 & $\mathrm{~s}$ & $\sigma_{11}$ & $v_{1}\left(\mathrm{CH}_{2}\right)$ \\
\hline 2942 & $\mathrm{~s}$ & $\sigma_{\perp}$ & $v_{a}\left(\mathrm{CH}_{2}\right)$ \\
\hline$\sim 3340$ & vs & $\sigma ?$ & $v(\mathrm{OH})$ \\
\hline 4010 & vw & $?$ & $\left\{\begin{array}{l}v_{a}\left(\mathrm{CH}_{2}\right)+v(\mathrm{CO})=4038 \\
v\left(\mathrm{CH}_{2}\right)+v(\mathrm{CO})=4006\end{array}\right.$ \\
\hline 4202 & vw & $\sigma$ & $\begin{array}{l}w(\mathrm{CH})+\delta(\mathrm{CH}+\mathrm{OH})=4216\end{array}$ \\
\hline 4255 & $\mathrm{vw}$ & $\sigma$ & $v_{a}\left(\mathrm{CH}_{2}\right)+\delta(\mathrm{CH}+\mathrm{OH})=4262$ \\
\hline 4329 & vw & $\sigma$ & $v_{1}\left(\mathrm{CH}_{2}\right)+\delta\left(\mathrm{CH}_{2}\right)=4340$ \\
\hline 4790 & vw & $\sigma$ & $v(\mathrm{OH})+\gamma_{v}\left(\mathrm{CH}_{2}\right)=4786$ \\
\hline 5688 & $\mathrm{vw}$ & $\sigma$ & $2 \times v(\mathrm{CH})=5680$ \\
\hline 5838 & $\mathrm{vw}$ & $\sigma$ & $v_{s}\left(\mathrm{CH}_{2}\right)+v_{a}\left(\mathrm{CH}_{2}\right)=5852$ \\
\hline 6330 & $\mathbf{v w}$ & $\sigma$ & \\
\hline 6700 & vw & $\sigma$ & $2 \times v(\mathrm{OH})=6680$ \\
\hline
\end{tabular}

rudimentary success at its interpretation. This is understandably a consequence of the increased complexity of the molecule and of some ambiguities as to its structure. In fact, despite the detailed studies which have been undertaken in recent years, many aspects of the spectrum still remain uncertain. Nevertheless a basically correct analysis seems to be emerging from this work. We will be concerned with outlining this analysis and discussing some of the problems which remain. 
The polarized infrared spectrum of an oriented specimen of PVA is shown in Fig. 11 [Krimm, Liang, and SutherLand (104)]. The band positions and polarization, given in Table 13, are derived from this and

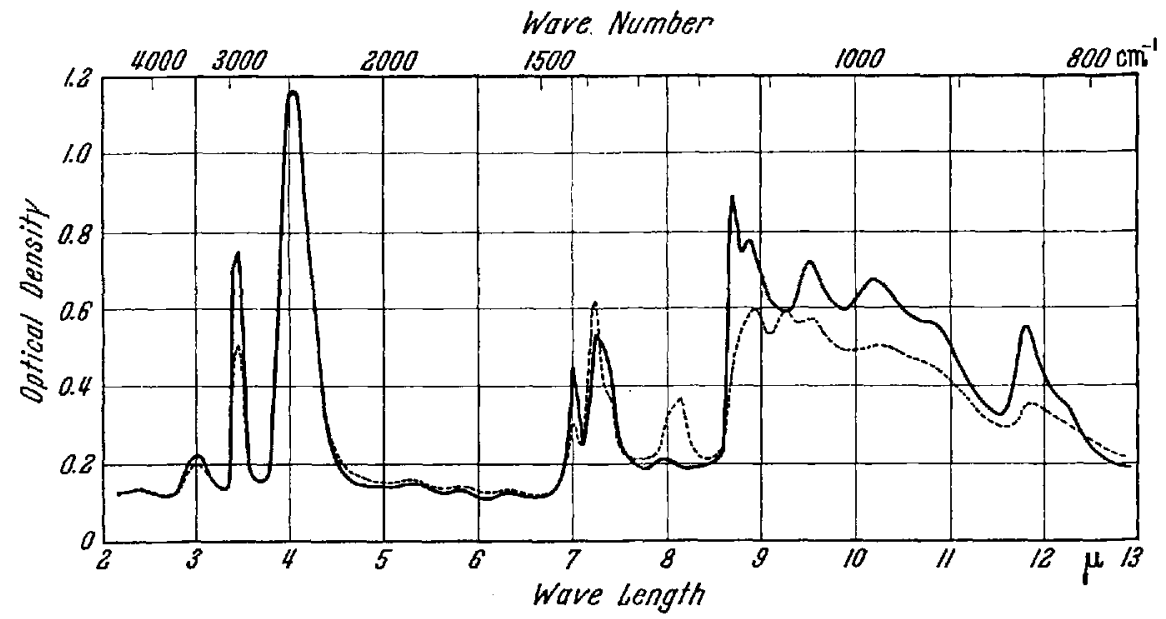

Fig. 12. Infrared spectrum of oriented polyvinyl alcohol-D. - - E perpendicular to stretching direction; -..-: E parallel to stretching direction [TADOKORO, SEKI, and NItTA (218)]

other studies [Glatt, Webber, Seaman, and Ellis (66); Glatt and Ellis (65); Tadokoro, SeKr, and NitTa (218)]. The subscripts $\perp$ and $\mid 1$ on the $\sigma$ bands at $2900 \mathrm{~cm}^{-1}$ and in the $800-1200 \mathrm{~cm}^{-1}$ region refer to the
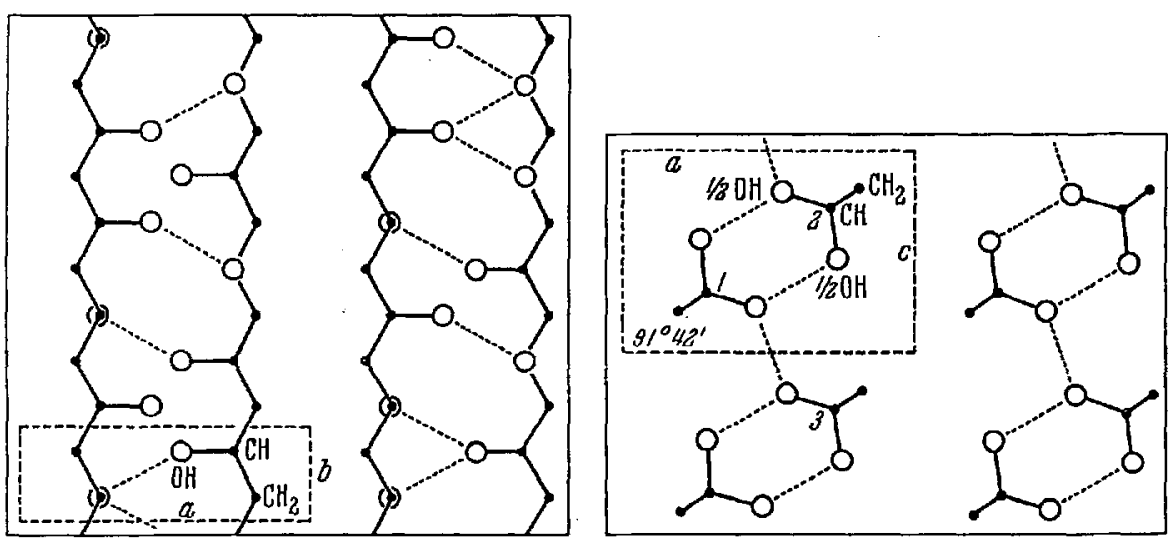

Fig. 13. Crystal structure of polyvinyl alcohol [Buwn (27)]

polarization with respect to the plane of the zig-zag carbon chain, and were obtained from spectra of doubly oriented specimens [AMBRose, Elliott, and Temple (6); Tadokoro, Sekr, Nitta, and Yamadera Fortschr. Hochpolym.-Forsch., Bd. 2 
(220)]. Spectra of PVA-D, ${ }_{-} \mathrm{CH}_{2} \mathrm{CHOD}_{\bar{n}}$, have also been obtained [TADOKoro, Seiki, and NitTa (217); Krimm, Liang, and Sutherland (104)], and constitute an important part of the analysis. A polarized spectrum of an oriented PVA-D sample is shown in Fig. 12 [TADOKORO, SEKI, and Nitta (218); TAdokoro (215a)]. No Raman spectra of PVA or related low molecular weight molecules are available.

The structure of PVA has been the subject of some controversy. X-ray diffraction studies have suggested two structures [MOONEY (140); BunN (27)], although the data seem to favor the Bunn structure. A careful analysis of the infrared spectrum [KRIMm, Liang, and SutherLaNd (104)], primarily via the dichroism of the $v(\mathrm{OH})$ and $\gamma_{w}(\mathrm{OH})$ modes, also supports the Bunn structure. We will therefore assume that this structure, shown in Fig. 13, is the correct one. It will be noted that it is characterized by a random placement of the $\mathrm{OH}$ groups along the chain (i.e., an atactic arrangement) and by hydrogen bonding between chains, as shown in the figure. From the standpoint of $x$-ray diffraction it is permissible to replace this structure by one containing two half-oxygen atoms at the $\mathbf{H}$ and $\mathrm{OH}$ positions of each $\mathrm{CHOH}$ group. It does not seem valid, however, to do this for purposes of spectroscopic analysis, as has been done [LiANG and Pearson (116)]. Such a procedure results in the prediction of skeletal modes which would not be expected to occur (e.g., the $\boldsymbol{v}_{a}(S)$ mode proposed by Liang and Pearson). Furthermore, the symmetry of the average structure deduced from $\mathrm{x}$-ray analysis is only correct if the locations of the $\mathrm{OH}$ hydrogen atoms are neglected. Assume an infinitely long planar zig-zag PVA chain on which the arrangement of the $\mathrm{OH}$ groups is atactic, and consider the mode which can be described as involving primarily a stretching of the $\mathrm{C}-\mathrm{O}$ bond. Defining the phase of the vibration in one monomer unit (e.g., by the length of the $\mathrm{C}-\mathrm{O}$ bond in relation to its equilibrium length), we can ask for the phase relation between such modes in adjacent units which will give rise to a large transition moment for the chain. It will be seen that only for a phase difference of zero (i.e., all $\mathrm{C}-\mathrm{O}$ bonds elongating simultaneously) is this moment large, otherwise it will be zero. For example, for the case of a phase difference of $\pi$ (a $\mathrm{C}-\mathrm{O}$ bond in one monomer unit elongating while that in the neighboring unit contracts, corresponding to the $v_{a}(S)$ mode mentioned above), since the location of $\mathrm{OH}$ groups is random, we expect to find as many $\mathrm{C}-\mathrm{O}$ bonds on one side of the zig-zag plane contracting as are elongating and the same for those bonds on the other side of the plane, resulting (for a very long chain) in a net transition moment of zero. The same should be true for any other phase difference. It appears that any interaction between the two chains in the unit cell will lead, because of the pseudo-symmetry characteristics of long atactic PVA chains in the crystal, to operation of the mutual exclusion rule, with all 
of the infrared active modes being derivable from those of a single chain. When, for a given mode, the motions on the two chains are in-phase (symmetric with respect to the pseudo center of inversion) the band will be Raman active; when the motions are out-of-phase the band will be infrared active. The primary consequence of this argument is that the skeletal modes must be determined by the $\mathrm{C}-\mathrm{C}-\mathrm{O}$ (monomer) unit and not by a $\mathrm{C}-\mathrm{C}-\frac{1}{2} \mathrm{O}$. follows.

On the basis of such a single chain analysis in which no rigorous symmetry is assigned to the structure we are forced to describe the modes and their polarization properties in terms of simple group vibrations. This leads to the following 17 modes: $\mathrm{CH}_{2}-v_{s}, v_{a}, \delta, \gamma_{w}, \gamma_{t}, \gamma_{r} ; \mathrm{CH}-\nu, \delta$, $\gamma_{w}$; OH $-v, \delta, \gamma_{w}$; skeletal $-v_{+}(0), \boldsymbol{\nu}_{+}(\pi), v(\mathrm{CO}), \delta(\mathrm{CO}), \gamma_{w}(\mathrm{CO})$. (For the $\mathrm{CH}, \mathrm{OH}$, and $\mathrm{CO}$ groups the $\delta$ mode represents a deformation perpendicular to the chain axis and the $\gamma_{w}$ mode a deformation parallel to this axis.) It will be recognized at the outset that this description is less than adequate, but it is not possible at present, in the absence of normal vibration calculations, to be more specific. We must therefore maintain caution in the interpretation placed on the above symbolic descriptions, recognizing the likelihood of interactions between $\mathrm{CO}$ and skeletal modes and between hydrogen vibrations of the $\mathrm{OH}$ group on the one hand and the $\mathrm{CH}$ and $\mathrm{CH}_{2}$ groups on the other. In the following discussion on the assignments we shall try to assess these interactions for the various modes.

Certain of the modes can be assigned quite readily on the basis of existing information. Consider first the $\mathrm{CH}_{2}$ modes. The general position, intensity, and polarization in the doubly oriented specimens of the bands at $2910 \mathrm{~cm}^{-1}$ and $2942 \mathrm{~cm}^{-1}$ identifies them with the $v_{8}\left(\mathrm{CH}_{2}\right)$ and $v_{a}\left(\mathrm{CH}_{2}\right)$ modes respectively. Similarly, the $1430 \mathrm{~cm}^{-1}$ band (located at $1428 \mathrm{~cm}^{-1}$ in PVA-D) can be identified with $\delta\left(\mathrm{CH}_{2}\right)$. The deviation of these frequencies from the values found in polyethylene and other polymers is an indication that these $\mathrm{CH}_{2}$ modes are not completely separable. The $v(\mathrm{OH})$ mode is clearly assignable to the broad band at $3340 \mathrm{~cm}^{-1}$, the value of this frequency being indicative of the existence of an essentially intermolecular type of hydrogen bonding [BELLAMY (13)]. On the basis of studies on alcohols [STUART and SUTHERLand (212)] and on PVA-D [KRIMm, LIang, and Sutherland (104)], the $\gamma_{w}(\mathrm{OH})$ mode can be assigned with fair certainty to the band at $630 \mathrm{~cm}^{-1}$. And finally, the $2840 \mathrm{~cm}^{-1}$ band is most probably to be associated with the $\nu(\mathrm{CH})$ mode while the $1235 \mathrm{~cm}^{-1} \pi$ band is likewise to be correlated with $\gamma_{w}(\mathrm{CH})$.

The assignment of the remaining bands presents somewhat greater problems. We will consider first the bands in the $1300-1500 \mathrm{~cm}^{-1}$ region of the spectrum. In earlier work [KRIMM, LIANG, and SUTHERLAND (104)] the 
assignments in this region were based on studies of PVA-D which did not include polarization measurements. Such measurements have since been made [TADokoro, Seki, and Nirta (218); TAdokoro (215a)], and seem to require some reconsideration of the earlier assignments. It will be seen from Fig. 12 that deuteration of the $\mathrm{OH}$ group in PVA leaves essentially three bands in this region: $1428 m(\sigma), 1383 s(\pi)$, and $1360(\sigma)$, the $1326 s(\sigma)$ band having been removed. It is most likely that all three of the former bands are associated with $\mathrm{CH}_{2}$ and $\mathrm{CH}$ modes. As we have seen, the $1428 \mathrm{~cm}^{-1}$ band is the $\delta\left(\mathrm{CH}_{2}\right)$ mode, found at $1430 \mathrm{~cm}^{-1}$ on PVA. A strong $\pi$ band comparable to the $1383 \mathrm{~cm}^{-1}$ band of PVA-D is found at $1446 \mathrm{~cm}^{-1}$ in PVA, and it would thus seem that these two bands have a common origin. Earlier arguments [KRIMM, LIANG, and SUTHERLAND $(103,104)]$ had suggested that the 1326 and $1430 \mathrm{~cm}^{-1}$ bands of PVA were coupled $\mathrm{CH}$ and $\mathrm{OH}$ bending modes, $\delta(\mathrm{CH}+\mathrm{OH})$, the $1383 \mathrm{~cm}^{-1}$ band of PVA-D being the uncoupled $\delta(\mathrm{CH})$ mode. If this is so, then it is indeed strange that the latter mode exhibits $\pi$ polarization. It would seem that a mode which basically has the character of $\delta(\mathrm{CH})$ should exhibit $\sigma$ polarization. An alternative assignment would be to assign the $1446 \mathrm{~cm}^{-1}$ band of PVA (and the $1383 \mathrm{~cm}^{-1}$ band of PVA-D) to $\gamma_{w}\left(\mathrm{CH}_{2}\right)$, and the 1376 and $1326 \mathrm{~cm}^{-1}$ bands of PVA to coupled $\delta(\mathrm{CH}+\mathrm{OH})$ modes, the former probably being mainly $\delta(\mathrm{CH})$ and the latter $\delta(\mathrm{OH})$. The weak $\sigma$ band at $1360 \mathrm{~cm}^{-1}$ in PVA-D would then represent the uncoupled $\delta(\mathrm{CH})$ mode. This would be consistent with the polarizations and relative intensities of the bands in PVA and PVA-D, and would imply that the position of $\gamma_{w}\left(\mathrm{CH}_{2}\right)$ is determined by interaction with $\delta(\mathrm{OH})$ (plus $\delta(\mathrm{CH})$ in PVA) or $\delta(\mathrm{OD})$, the latter interaction presumably being smaller than the former. Although we will propose this set of assignments, it is clear that the study of the ${ }_{-}^{\prime} \mathrm{CD}_{2} \mathrm{CHOH}_{\bar{n}}$ and ${ }_{-}\left(\mathrm{CH}_{2} \mathrm{CDOH}_{\bar{n}}\right.$ polymers is essential to a satisfactory resolution of this problem.

Some problems also remain in the assignment of the bands in the $800-1200 \mathrm{~cm}^{-1}$ region. On the basis of the above single chain analysis we would expect the $v(\mathrm{CO}), \gamma_{r}\left(\mathrm{CH}_{2}\right), v_{+}(0)$, and $v_{+}(\pi)$ modes to be found here. It seems that most of these modes can now be identified with fair certainty; the main problems are the additional bands occurring in this region, The $\nu(\mathrm{CO})$ mode of a chain unit should exhibit $\sigma$ polarization, with slightly higher intensity of absorption perpendicular to the zig-zag plane than parallel. This and other arguments [KRIMM, Liang, and SUTHERLAND (104)] lead to the assignment of the $1096 \mathrm{~cm}^{-1}$ band to $v(\mathrm{CO})$ without too much ambiguity. Similarly, the frequency and the polarization with respect to the zig-zag plane of the $825 \mathrm{~cm}^{-1}$ band make it highly probable that this band is to be assigned to $\gamma_{r}\left(\mathrm{CH}_{2}\right)$. The skeletal modes are more difficult to identify. On the basis of the presence of a $\pi$ band at $1083 \mathrm{~cm}^{-1}$ in PVA-D, we can assign the weak $1085 \mathrm{~cm}^{-1}$ 
$\pi$ (?) band of PVA to the mode corresponding to $\nu_{+}(\pi)$ in polyethylene. Calculations on ethyl alcohol [CROss and VAN VLECK (43)] indicate that the $v$ (CC) mode should occur at about $910 \mathrm{~cm}^{-1}$, and on this basis it is probable that the mode corresponding to $\nu_{+}(0)$ in polyethylene can be associated with the $915 \mathrm{~cm}^{-1}$ band of PVA. It also has the correct polarization with respect to the plane of the carbon zig-zag chain. The remaining bands which require interpretation are those at 850 and 1141 $\mathrm{cm}^{-1}$. The former shows no preferred polarization with respect to the zig-zag plane in a doubly oriented specimen [TADOKORO, SEKI, NITTA, and YAMADERA (220)], and it is possible that it may be associated with a $\gamma_{r}\left(\mathrm{CH}_{2}\right)$ mode of chains in the noncrystalline regions, which are known to be oriented along the stretching direction [MACGillavRY (122)]. This could be confirmed by studies on $\left.{ }^{-} \mathrm{CD}_{2} \mathrm{CHOH}\right)_{\bar{n}}$.

The origin of the $1141 \mathrm{~cm}^{-1}$ band has been the subject of much discussion. It has been established [TADOKORO, SEKI, and NitTa (216); Krimm, Liang, and Sutherland (704); Tadokoro, Kôzar, Sekr, and NitTa (219); TADOKORO (215b)] that the intensity of this band, and presumably only this one, increases significantly when the sample is heated, a treatment which appears to increase the degree of crystallinity. It is also observed [HAAS (70)] that the intensity of this band increases when a sample is humidified, a treatment which is thought to result in an increase in the relative amount of ordered regions. This observation seems to preclude the assignment of the $1141 \mathrm{~cm}^{-1}$ band to an ether linkage C-O- $\mathrm{C}^{\prime}$, as has been suggested [KRIMM, Liang, and SutherLaNd (104)]. It has been suggested [TADokoro, Sekr, and NitTa (218); TadoKORO $(215 b)]$ that this band arises from a symmetric $\mathrm{C}-\mathrm{C}$ stretching mode, which would be consistent with its polarization parallel to the zig-zag chain, but this assignment presents difficulties too. In particular, it places such a skeletal mode at what seems to be much too high a frequency, and the skeletal vibration involved, viz., $v_{+}(0)$, is already adequately assigned to the band at $915 \mathrm{~cm}^{-1}$.

Several arguments suggest that the $1141 \mathrm{~cm}^{-1}$ band may be a $v(\mathrm{CO})$ mode. Although this band is clearly associated with the crystalline regions, there is reason to believe that it is not correlated with the assumed structure. If it were, and its intensity increase on crystallization were a result of the presence of more crystalline chains, then we should expect other bands which are also due to the planar zig-zag chain conformation to similarly increase in intensity. There is no evidence that this is the case. It would therefore seem that this band is associated with a structure that is more likely when the chain is in the crystalline state, a structure which represents only a small modification of that proposed by BunN. We wish to suggest that this band may be associated with the $v(\mathrm{CO})$ mode 
of a portion of a chain in which an intramolecular hydrogen bond is formed between two neighboring $\mathrm{OH}$ groups that are on the same side of the plane of the carbon chain. The transition moment for this vibration, which involves at least a $\mathrm{C}-\mathrm{CO}-\mathrm{C}-\mathrm{CO}$ segment, could easily be primarily parallel to the plane of the zig-zag. This suggestion is supported by recent work on pentane-2,4-diol [NAGaI, KuRIBayashi, SHIRAKI, and UkITA (141a)]. For the isomer in which it appears that both $\mathrm{OH}$ groups are on the same side of the plane of the carbon atoms, it is found that a band at $1157 \mathrm{~cm}^{-1}$ (similar in appearance to the $1141 \mathrm{~cm}^{-1}$ band of PVA) increases markedly in intensity under conditions (in dilute $\mathrm{CS}_{2}$ solutions) that lead to a preponderance of intramolecular over intermolecular hydrogen bonds. Also, it appears that both the 1141 and $1096 \mathrm{~cm}^{-1}$ bands move to higher frequencies on deuteration, the former to $1148 \mathrm{~cm}^{-1}$ and the latter to $1122 \mathrm{~cm}^{-1}$ in PVA-D, suggesting a similarity in the nature of the vibrations associated with the two bands. The increase in frequency on deuteration is not unreasonable if the $v(\mathrm{CO})$ mode is coupled with other vibrations of the $\mathrm{COH}$ group, as is evidenced by studies on alcohols [STUART and SutherLand (212a)]. For example, the smaller anharmonicities associated with the motion of $\mathrm{D}$ atoms as compared to $\mathrm{H}$ atoms could, since anharmonicity constants are usually negative, well result in the increases observed. This suggested assignment could be checked by a careful study of changes which occur elsewhere in the spectrum associated with the change in intensity of the $1141 \mathrm{~cm}^{-1}$ band. Thus, it might be anticipated that $v(\mathrm{OH})$ modes associated with intramolecular hydrogen bonds should be detected, and possibly also changes associated with $v(\mathrm{OH} \cdots \mathrm{O})$ modes (see below). A C $-\mathrm{C}$ stretching mode associated with such a group might also be anticipated, and perhaps this is the origin of the $1040 \mathrm{~cm}^{-1}$ band.

Of the remaining modes to be assigned, $\gamma_{t}\left(\mathrm{CH}_{2}\right)$ is not identifiable with any certainty. The $\delta(\mathrm{CO})$ and $\gamma_{w}(\mathrm{CO})$ modes are probably to be associated with the bands at $480 \mathrm{~cm}^{-1}$ and $410 \mathrm{~cm}^{-1}$ respectively. It seems not unlikely that the $185 \mathrm{~cm}^{-1}$ band, and perhaps also the shoulder at $135 \mathrm{~cm}^{-1}$, arises from a $\nu(\mathrm{OH} \cdots \mathrm{O})$ vibration [Gross (69)], i.e., a vibration in which the $\mathrm{O} \cdots \mathrm{O}$ distance changes but not the $\mathrm{OH}$ distance. Many of the high frequency bands seem to be interpretable in terms of binary combinations, and some of these are suggested.

It will be seen that many features of the PVA spectrum can be interpreted moderately well. On the other hand, the assignments of certain of the bands still require confirmation. This can probably be accomplished by a study of other deuterated species of the polymer, with substitution on the $\mathrm{CH}$ and $\mathrm{CH}_{2}$ groups. The value of such a study is evident in this case, since, for example, if the suggested assignment of the $1141 \mathrm{~cm}^{-1}$ band could be confirmed a deeper insight into the structure of this polymer in 
the crystalline state would have been achieved. Similarly, the relatively large frequency splitting between the $\sigma$ and $\pi$ components of the $v(\mathrm{OH}) \delta(\mathrm{OH})$, and $\gamma_{w}(\mathrm{OH})$ modes, which has not yet been convincingly explained, probably also reflects important aspects of thestructure and internal forces.

\section{E. Polypropylene}

The infrared spectrum of polypropylene, $-\left(\mathrm{CH}_{2} \mathrm{CH}_{\bar{n}}\right.$, has not been the subject $\mathrm{CH}_{3}$

of a very detailed investigation. Although infrared and Raman data have recently been published, and studies have been undertaken on crystalline bands, no extensive studies on deuterated species have as yet been published. We will, nevertheless, undertake to outline the problem of the assignments for polypropylene, since this polymer is structurally typical of a large class of isotactic polymers. We will try to show how the infrared spectrum of this polymer can shed significant light on its structure.

Polarized infrared spectra of oriented specimens of isotactic polypropylene are shown in Fig. 14 [Peraldo (167)]. The band positions, polarizations, and relative intensities are given in Table 14, together with Raman data [TOBIN (231)] and information on the phase of origin of the bands [NatTa (149); Pokrovsky and VolkenShtein (178); ABE and Yanagisawa (1)]. Some preliminary data on partially deuterated polypropylenes are available [PERALDo (167); NATTA (150)], but no detailed spectra have as yet been published.

The structure of isotactic polypropylene has been investigated by $\mathrm{x}$-ray diffraction [Natta and Corradini (151); NatTa, CorRadini, and Cesari (155); Natta and
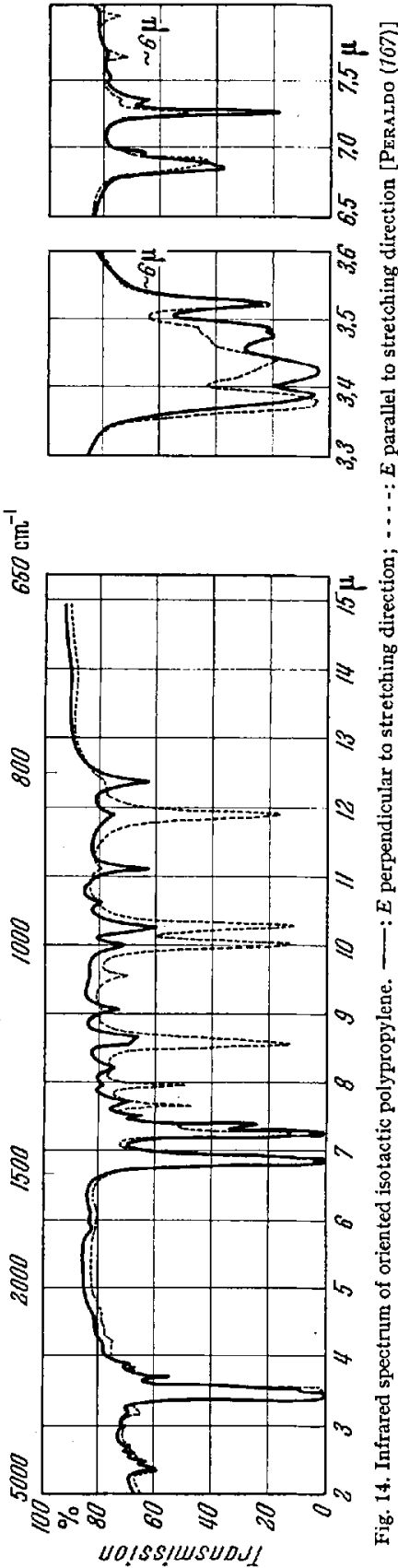
Table 14. Vibrational Spectrum of Polypropylene

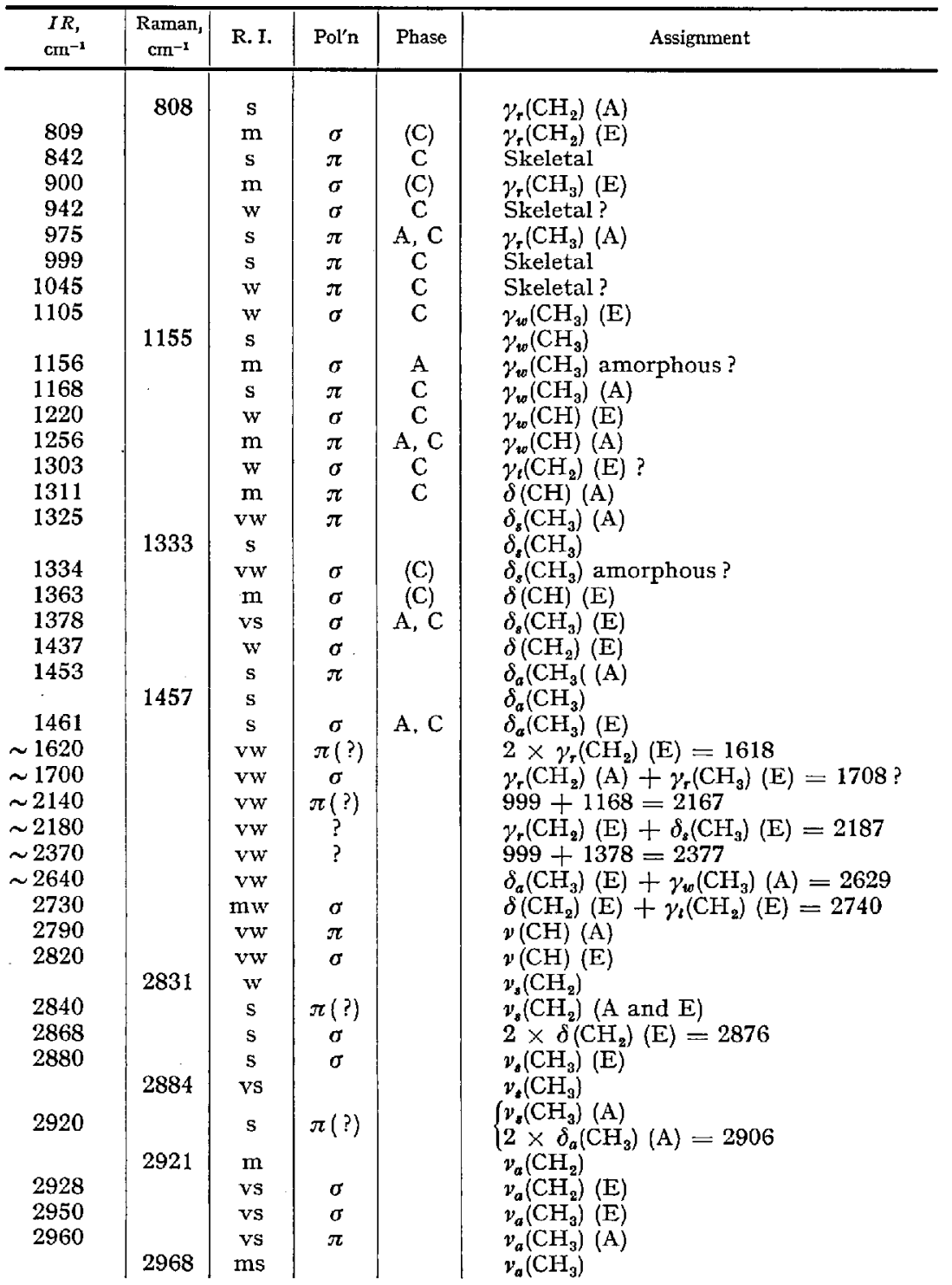

Corradini (154a)]. These studies indicate that the polymer chain is helical, with three monomer units in the repeat distance of $6.50 \mathrm{~A}$ along the fiber axis. Thus, the only symmetry element possessed by the structure is a 3-fold screw axis. The chain is polar, since the axis of the methyl group makes an angle of about $72^{\circ}$ with the helix axis (the $\mathrm{C}-\mathrm{C}-\mathrm{C}$ angle in the 
chain is $\left.114.5^{\circ}\right)$. Although the unit cell contains more than one chain [Natta, Corradini, and Cesari (155); Keith, Padden, Walter, and WYCKOFr (86)], it is most fruitful at this stage of the analysis to deal only with the spectrum of a single chain. As has been shown [PERALDO (167)], the normal modes are expected to split up into 25 of species $A$, in which the phase difference between motions in neighboring groups is zero and the transition moment is parallel to the helix axis, and 52 of species $\mathrm{E}$, in which the corresponding phase difference is $\pm 2 \pi / 3$ and the transition moment is perpendicular to the axis (the latter consisting of 26 degenerate pairs). Modes in either species are both infrared and Raman active. The chain modes can be described in terms of modes of the individual groups [PERALDo (167)], as was done for polytetrafluoroethylene. Although this may not be a valid approximation in some cases, it is justified as a starting point for the analysis (at least until it is demonstrated to be invalid). Such a classification of the chain modes is shown in Table 15. In addition, on the Table 15. Classification of Normal Modes of

\begin{tabular}{|c|c|c|}
\hline & Vibration & $I_{A} / I_{E}$ \\
\hline $\begin{array}{l}\text { Hydrogen Modes, } \\
\text { A and E: }\end{array}$ & $\begin{array}{l}v(\mathrm{CH}) \\
v_{s}\left(\mathrm{CH}_{2}\right) \\
v_{a}\left(\mathrm{CH}_{2}\right) \\
v_{s}\left(\mathrm{CH}_{3}\right) \\
v_{a}\left(\mathrm{CH}_{3}\right)_{i} \\
v_{a}\left(\mathrm{CH}_{3}\right)_{0} \\
\delta(\mathrm{CH}) \\
\delta\left(\mathrm{CH}_{2}\right) \\
\delta_{a}\left(\mathrm{CH}_{3}\right) \\
\delta_{a}\left(\mathrm{CH}_{3}\right)_{t} \\
\delta_{a}\left(\mathrm{CH}_{3}\right)_{0} \\
\gamma_{w}\left(\mathrm{CH}^{2}\right) \\
\gamma_{w}\left(\mathrm{CH}_{2}\right) \\
\gamma_{w}\left(\mathrm{CH}_{3}\right) \\
\gamma_{r}\left(\mathrm{CH}_{2}\right) \\
\gamma_{r}\left(\mathrm{CH}_{3}\right) \\
\gamma_{l}\left(\mathrm{CH}_{2}\right) \\
\gamma_{t}\left(\mathrm{CH}_{3}\right)\end{array}$ & $\begin{array}{r}0.8: 2.1 \\
2.9: 1.6 \\
0: 2.3 \\
0.8: 2.1 \\
1.6: 1.9 \\
6.5: 0.6 \\
1.6: 1.9 \\
2.9: 1.6 \\
0.8: 2.1 \\
1.6: 1.9 \\
6.5: 0.6 \\
6.5: 0.6 \\
6.5: 0.6 \\
6.5: 0.6 \\
0: 2.3 \\
1.6: 1.9 \\
-\end{array}$ \\
\hline $\begin{array}{l}\text { Skeletal Modes: } \\
\mathrm{CH}_{3} \text { Group }\end{array}$ & $\begin{array}{c}v\left(\mathrm{C}-\mathrm{CH}_{3}\right) \mathrm{A}, \mathrm{E} \\
\delta\left(\mathrm{C}-\mathrm{CH}_{3}\right) \mathrm{A}, \mathrm{E} \\
\gamma_{t o}\left(\mathrm{C}-\mathrm{CH}_{3}\right) \mathrm{A}, \mathrm{E} \\
v_{1}-v_{4} \mathrm{~A} \\
v_{5}-v_{9} \mathrm{E}\end{array}$ & $\begin{array}{l}0.8: 2.1 \\
1.6: 1.9 \\
6.5: 0.6\end{array}$ \\
\hline
\end{tabular}
basis of the assumed structure it is possible to compute the approximate intensity ratio of the $\mathrm{A}$ to the $\mathrm{E}$ species chain vibrations corresponding to a given group mode. We show this ratio also in Table 15 for some of the hydrogen and skeletal modes. It is more difficult to specify for the remaining skeletal vibrations since the form of these modes is unknown. (The separation of the skeletal modes into those of the $\mathrm{CH}_{3}$ group and those of the carbon chain backbone is open to question.) The $i$ and $o$ on the $v_{a}\left(\mathrm{CH}_{3}\right)$ and $\delta_{a}\left(\mathrm{CH}_{3}\right)$ modes indicate in-plane and out-of-plane, the plane referred to being that of $\mathrm{H}-\mathrm{C}-\mathrm{CH}_{3}$. We expect the degenerate vibrations of the isolated $\mathrm{CH}_{3}$ group to be split in the polymer, the transition moments of the components probably being determined by the local pseudo plane of symmetry referred to above.

Several interesting observations can be made from the $I_{A} / I_{E}$ ratios. Thus, for example, the intensities of $v_{a}\left(\mathrm{CH}_{2}\right)(\mathrm{A})$ and $\gamma_{r}\left(\mathrm{CH}_{2}\right)(\mathrm{A})$ should 
be zero. For some of the modes $\left[v(\mathrm{CH}) ; v_{s}\left(\mathrm{CH}_{3}\right), \delta_{s}\left(\mathrm{CH}_{3}\right)\right.$, and $\left.\boldsymbol{v}\left(\mathrm{C}-\mathrm{CH}_{3}\right)\right]$ the $A$ component should be weaker than the $E$ component; for others $\left[\nu_{a}\left(\mathrm{CH}_{3}\right)_{i}, \delta(\mathrm{CH}), \delta_{a}\left(\mathrm{CH}_{3}\right)_{i}, \gamma_{r}\left(\mathrm{CH}_{3}\right)\right.$, and $\left.\delta\left(\mathrm{C}-\mathrm{CH}_{3}\right)\right]$ the intensities of the two components should be approximately equal; for $v_{s}\left(\mathrm{CH}_{2}\right)$ and $\delta\left(\mathrm{CH}_{2}\right)$ the $A$ component should be somewhat stronger than the $E$ component; while for another group $\left[v_{a}\left(\mathrm{CH}_{3}\right)_{0}, \delta_{a}\left(\mathrm{CH}_{3}\right)_{0}, \gamma_{w}(\mathrm{CH}), \gamma_{w}\left(\mathrm{CH}_{2}\right), \gamma_{w}\left(\mathrm{CH}_{3}\right)\right.$, and $\left.\gamma_{w}\left(\mathrm{C}-\mathrm{CH}_{3}\right)\right]$ the $A$ component should be very much stronger than the $E$ component. If the structure is correct, and the assumption of a single chain analysis is valid, then these relationships should help identify the bands. Conversely, if the modes can be independently assigned, say with the help of studies on deuterated species, then the analysis of the $I_{A} / I_{E}$ ratios should permit something to be said about the structure. It will be seen that neither case is clearly supported by the presently available data, so that further study is definitely required.

We consider first the hydrogen stretching modes. If we require that $v_{a}\left(\mathrm{CH}_{2}\right)(A)$ be zero, and that $\nu(\mathrm{CH})(A)$ (by analogy with polymer spectra) be weak, then we are left with three $\pi$ bands to be assigned to four $A$ modes. This suggests that perhaps the splitting between $\nu_{a}\left(\mathrm{CH}_{3}\right)_{i}$ and $v_{a}\left(\mathrm{CH}_{3}\right)_{0}$ is too small to be observed. If we assume this to be the case, then with the help of data on the general regions in which the frequencies are to be expected [ShEPPARD and Simpson (195)] we can make the following assignments: $2960-v_{a}\left(\mathrm{CH}_{3}\right)(A), 2920-v_{s}\left(\mathrm{CH}_{3}\right)(A)$, and $2840-v_{s}\left(\mathrm{CH}_{2}\right)(A)$. For the $E$ components we would then probably have: $2950-v_{a}\left(\mathrm{CH}_{3}\right)(E), 2928-v_{a}\left(\mathrm{CH}_{2}\right)(E), 2880-v_{s}\left(\mathrm{CH}_{3}\right)(E)$, and $2840-$ $\nu_{s}\left(\mathrm{CH}_{2}\right)(E)$. The $\nu(\mathrm{CH}) A$ and $E$ modes are perhaps to be assigned to the very weak bands at 2790 and $2820 \mathrm{~cm}^{-1}$ respectively. The band at $2868 \mathrm{~cm}^{-1}$ may be the overtone of the $1437 \mathrm{~cm}^{-1}$ band, and the $2920 \mathrm{~cm}^{-1}$ band may contain a contribution from the overtone of the $1453 \mathrm{~cm}^{-1}$ band. Even with these possibilities the intensity ratios do not seem to agree too well with those predicted. It must be remembered though that the calculated ratios are based on transition moments which are simply related to the symmetry of each group, and this may not be valid. These results therefore indicate that the assignments must be confirmed by deuteration studies. Thus, for example, the $2920 \mathrm{~cm}^{-1} \pi$ band is at a position which would be consonant with an assignment to $\boldsymbol{v}_{a}\left(\mathrm{CH}_{2}\right)$, but its intensity seems to be much too high on the basis of prediction, even considering the presence of some amorphous material. If deuteration studies should indicate that this is such a $\mathrm{CH}_{2}$ mode, then it would seem that some modification in the proposed structure would have to be considered.

In the hydrogen bending region, deuteration studies [PERALDO (167); NATTA (750)] indicate that the $1437 \mathrm{~cm}^{-1} \sigma$ band is associated with the $\mathrm{CH}_{2}$ group, thus suggesting its assignment to $\delta\left(\mathrm{CH}_{2}\right)(E)$. This would then require that the $1453 \pi$ and $1461 \sigma$ bands be assigned to $\delta_{a}\left(\mathrm{CH}_{3}\right)(A)$ and 
$\delta_{a}\left(\mathrm{CH}_{3}\right)(E)$ respectively (the splitting of the degenerate mode being neglected). This is a moderately satisfactory assignment, except that the intensity ratio is not in accord with that predicted by the structure. Again, however, we have made the simplest assumptions about the transition moment directions, and these may not be correct. The $\delta\left(\mathrm{CH}_{2}\right)(A)$ mode is not identified, but since the $\delta\left(\mathrm{CH}_{2}\right)$ modes are much weaker than the $\delta_{a}\left(\mathrm{CH}_{3}\right)$ modes [FRANCIS (57)] it is possible that $\delta\left(\mathrm{CH}_{2}\right)(A)$ is overlapped by the stronger $\delta_{a}\left(\mathrm{CH}_{3}\right)$ bands. The very strong $\sigma$ band at $1378 \mathrm{~cm}^{-1}$ is undoubtedly the $\delta_{8}\left(\mathrm{CH}_{3}\right)(E)$ mode. The $\delta_{s}\left(\mathrm{CH}_{3}\right)(A)$ mode is expected to be much weaker, and may be associated with the very weak $\pi$ band at $1325 \mathrm{~cm}^{-1}$. Again the intensity ratio is quite different from that expected.

The assignment of the remaining hydrogen modes and the skeletal modes is far from unambiguous on the basis of existing data. Therefore the assignments to be proposed for these modes must be taken as providing no more than a preliminary working framework, to be verified or modified by further studies. We will assume the absence of $\gamma_{r}\left(\mathrm{CH}_{2}\right)(A)$ in the spectrum, as predicted from the model. Then the assignment of $\gamma_{r}\left(\mathrm{CH}_{2}\right)(E)$ to the $809 \mathrm{~cm}^{-1}$ band is perhaps likely. It may be that the $808 \mathrm{~cm}^{-1}$ Raman band is assignable to $\gamma_{r}\left(\mathrm{CH}_{2}\right)(A)$, since the $A$ species modes should be strong in the Raman spectrum. The strong $\pi$ bands at 1168 and $975 \mathrm{~cm}^{-1}$ are undoubtedly the deformation modes of the $\mathrm{CH}_{3}$ groups, and many arguments suggest the assignment of the former to $\gamma_{w}\left(\mathrm{CH}_{3}\right)(A)$ and the latter to $\gamma_{r}\left(\mathrm{CH}_{3}\right)(A)$. The corresponding $E$ modes cannot be identified with any certainty at present; bands at $1105 \mathrm{~cm}^{-1}$ and $900 \mathrm{~cm}^{-1}$ are suggested for these assignments. By analogy with the spectra of other polymers, assignments for $\delta(\mathrm{CH})$ and $\gamma_{w}(\mathrm{CH})$ can be made. The assignment of $\gamma_{t}\left(\mathrm{CH}_{2}\right)(E)$ to the $1303 \mathrm{~cm}^{-1}$ band is based on analogy with the results for polyethylene. The $\gamma_{w}\left(\mathrm{CH}_{2}\right)$ modes do not seem to be identifiable. More by default than otherwise, the remaining bands may be assigned to skeletal modes, although it must be admitted that in two cases ( 842 and $999 \mathrm{~cm}^{-1}$ ) the intensities are surprisingly high.

It will be apparent from the above discussion that a satisfactory set of assignments has not yet been achieved for the polypropylene spectrum. In most cases this is because the basic nature of the mode is unknown. Studies on partially and fully deuterated polypropylenes would answer these questions, and will clearly have to be undertaken in order for the final results to be more certain. When this has been done it should then be possible to evaluate more meaningfully the significance of the deviations between the observed and calculated $I_{A} / I_{E}$ ratios. As we have noted, these ratios are determined by the structure, if we make simple assumptions about the group transition moment directions. The deviations imply that either these assumptions are not valid or that the structure requires 


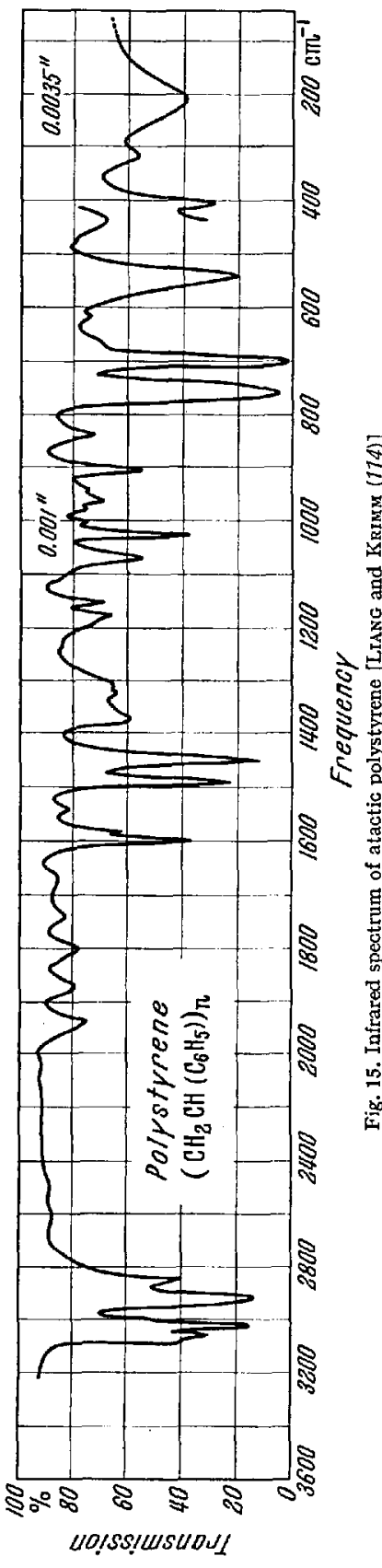

modification. (Another possibility is that the single chain analysis may not be adequate.) Which of these possibilities is the more likely could be better determined after the assignments are more securely known. These considerations illustrate the way in which a complete analysis of the spectrum of a polymer can serve to illuminate significantly various aspects of the structure and properties of the molecule.

\section{F. Polystyrene}

The spectrum of polystyrene, $\left(\mathrm{CH}_{2} \mathrm{CH}_{i n}\right.$, $\mathrm{C}_{6} \mathrm{H}_{5}$ provides a good illustration of the importance of detailed studies on small molecules, in this case benzene and monosubstituted benzenes, in the analysis of the spectrum of a polymer. It also demonstrates the feasibility of applying local symmetry considerations to the analysis of the spectrum. Early work was done on the atactic polymer. It will be seen that this analysis permits us to draw some conclusions about the structure of isotactic polystyrene.

The infrared spectrum of a sample of atactic polystyrene is shown in Fig. 15 [LIANG and KRIMM (114)]. The band positions are given in Table 16, on the left-hand side of the "Infrared" column. Raman data for atactic polystyrene [SIGNER and WEILER (198); PALM (163)] are also included in the table. Polarized spectra of oriented specimens of isotactic polystyrene have recently been published [Tadokoro, Nishiyama, NozaKura, and Murahashi (227); Tadokoro, Nozakura, Kitazawa, Yasuhara, and MURAHAshi (222); Takeda, IImURA, YAmada, and Imamura (222a); Morero, Mantica, Ciampelli, and Sianesi $(140 a)$ ]. The spectrum of the isotactic polymer differs slightly from that of the atactic polymer, and these differences (above $500 \mathrm{~cm}^{-1}$ ) are shown 
Table 16. Vibrational Spectrum of Polystyrene

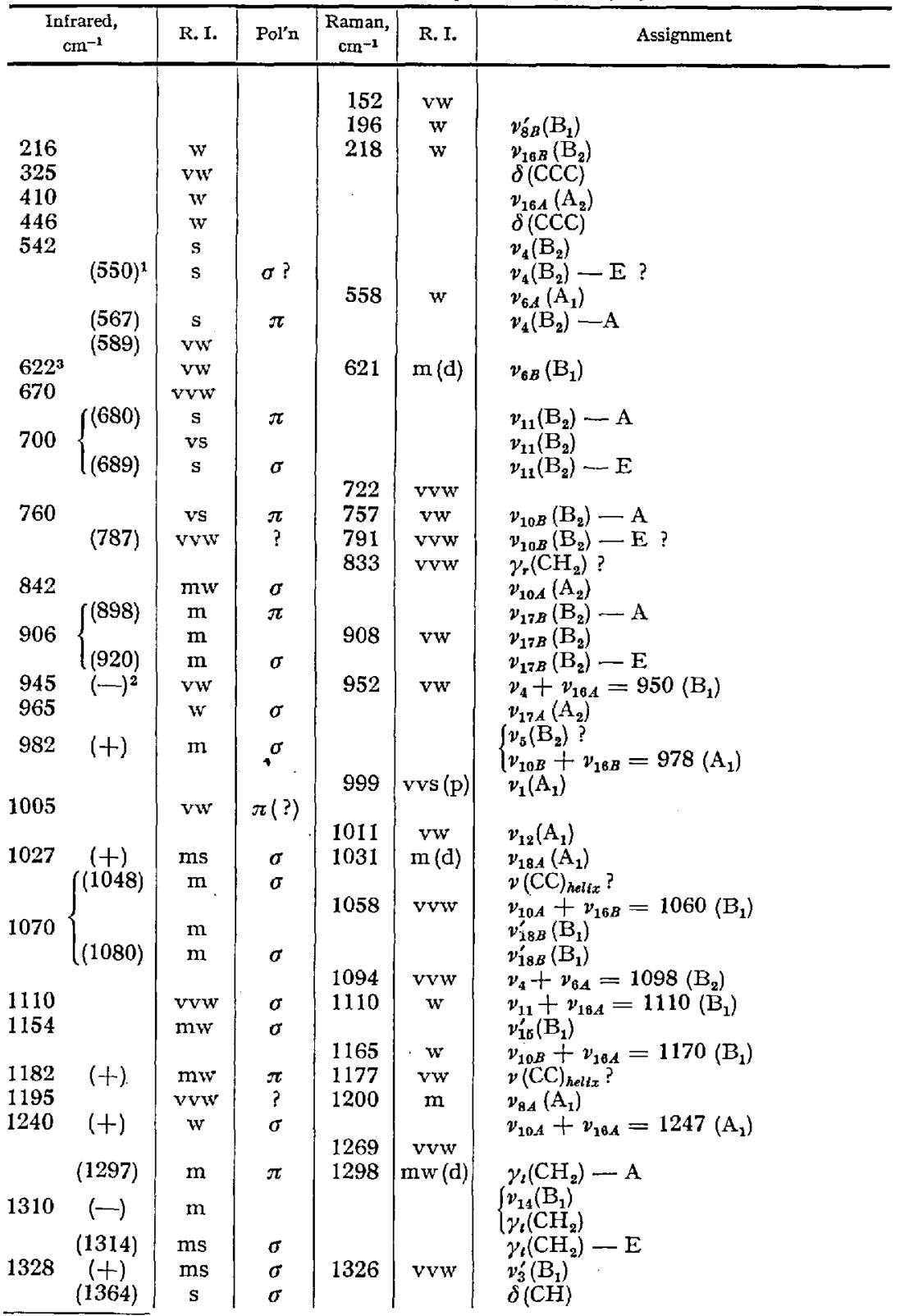

( ) : new bands, appearing in the spectrum of isotactic polystyrene.

( $(+)(-)$ : bands of atactic polystyrene which increase or decrease in intensity in the isotactic polymer.

3 Other bands are common to the spectra of both polymers. 
Table 16. (Continuation)

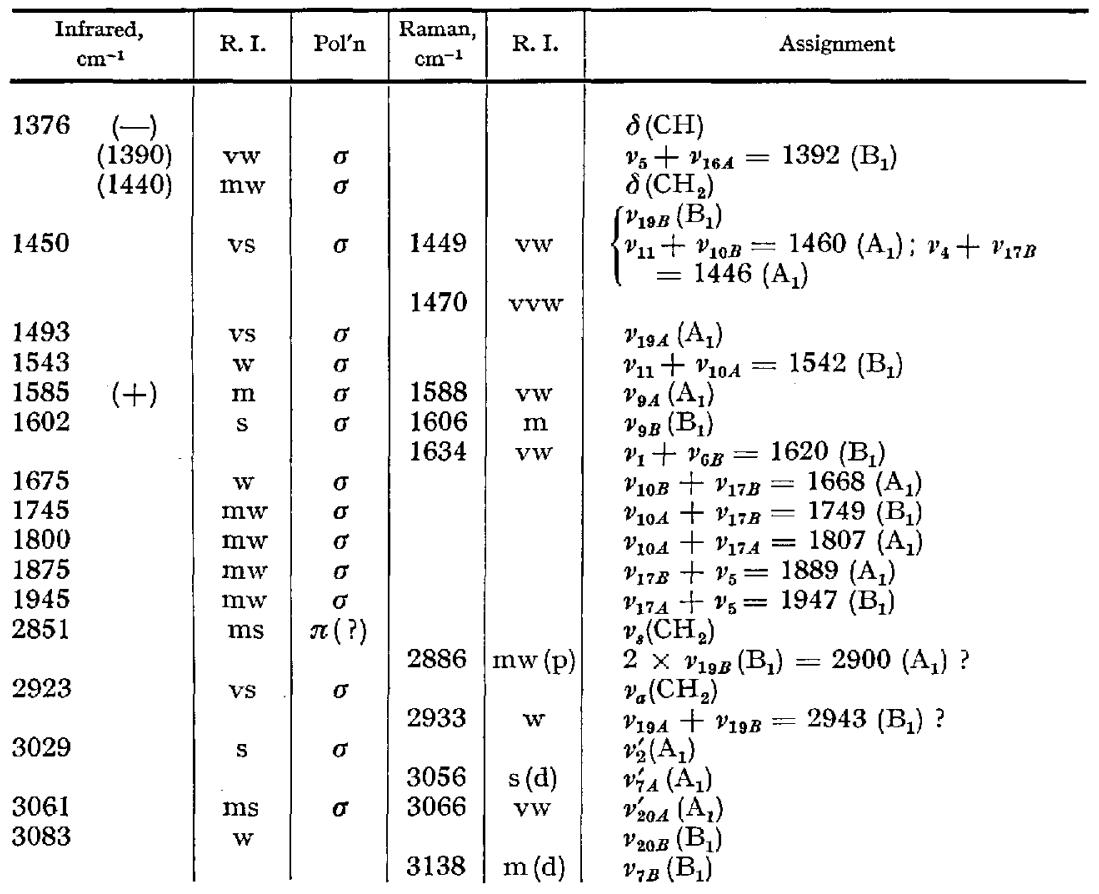

in Table 16 by enclosing the different band positions for the isotactic polymer in parentheses. In some cases (e.g., at 550 and $1297 \mathrm{~cm}^{-1}$ ) new bands appear in the isotactic polymer; in others (e.g., at 700,906 , and $1070 \mathrm{~cm}^{-1}$ ) a band of the atactic polymer is split in the spectrum of the isotactic polymer; and in still others (e.g., at 945,985 , and $1027 \mathrm{~cm}^{-1}$ ) a band of the atactic polymer is either enhanced $(+)$ or diminished $(-)$ in intensity in the spectrum of the isotactic polymer. The dichroism of the bands of isotactic polystyrene are also shown in the table.

The structure of isotactic polystyrene has been studied by means of $x$-ray diffraction [NATTA and CoRRADINI $(152,153)]$. It is found that the chain configuration is helical, with three monomer units per repeat exactly as in polypropylene. The only difference is that the chain repeat of $6.65 \mathrm{~A}$ in polystyrene implies an opening of the CCC bond angles in the chain to $116.5^{\circ}$. The orientation of the benzene ring is apparently not settled. In the above structure, if the axis of the benzene ring were perpendicular to the helix axis, then the plane of the benzene ring would be almost exactly perpendicular to the helix axis. In a variation of this structure proposed on the basis of stereochemical considerations [BunN and Howells (30)], and shown in Fig. 16, the plane of the benzene ring 
would be tipped about $25^{\circ}$ away from the above position. The polarized infrared spectra permit a choice to be made between these two structures. As has been shown [LIANG and Krimm (114)], the bands at 700 and $760 \mathrm{~cm}^{-1}$ are associated with transition moments perpendicular to the plane of the benzene ring, those at 1027 and $1493 \mathrm{~cm}^{-1}$ correspond to modes with transition moments parallel to the axis of the ring, and the $1450 \mathrm{~cm}^{-1}$ band is associated with a mode whose moment is in the plane of ring and perpendicular to the ring axis. As expected, the first two bands show $\pi$ dichroism while the others show $\sigma$ dichroism. The $\pi$ dichroism, however, is relatively small, and this would seem to favor the orientation of the benzene rings shown in Fig. 16. This is supported by examination of the $\sigma$ dichroism of the above bands. If the orientation of the benzene rings were that proposed by NATTA and CoRRADINI, it would be expected that the $1450 \mathrm{~cm}^{-1}$ band would exhibit a higher $\sigma$ dichroism than that of the 1027 and $1493 \mathrm{~cm}^{-1}$ bands. (The dichroism of the $1450 \mathrm{~cm}^{-1}$ band can be examined in poly$\alpha, \beta, \beta$-trideuterostyrene $\left[\mathrm{TA}_{\mathrm{A}}-\right.$

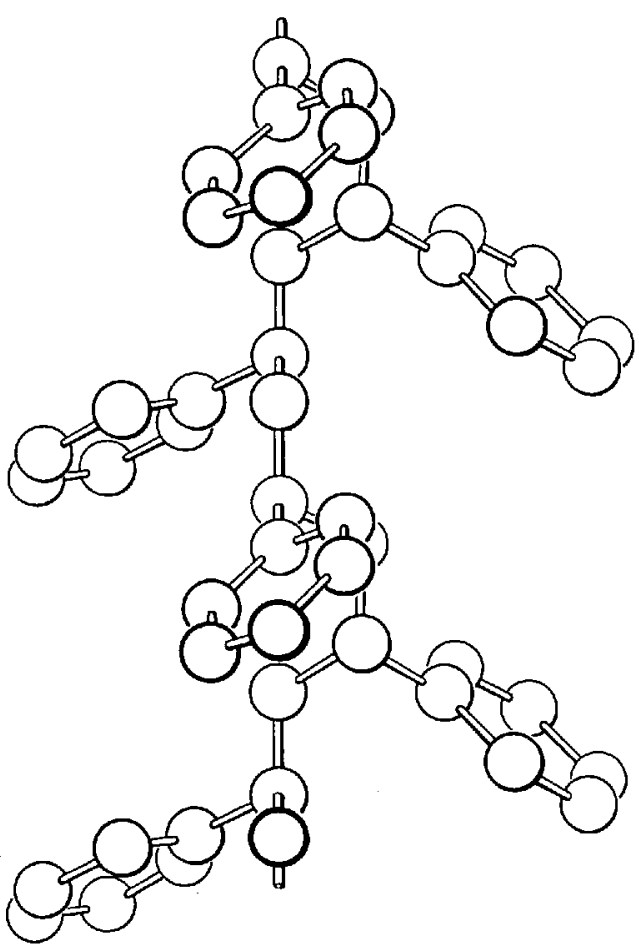
Fig. 16. Chain structure of isotactic polystyrene [BuNN and
HoweLIs (30)] DOKORO, NishIYAMA, NOZAKURA, and MURAHASHI (227)], where overlap with the $\delta\left(\mathrm{CH}_{2}\right)$ mode is avoided. The spectra show that the $\sigma$ dichroisms are comparable, thus favoring the tilt of the benzene rings shown in Fig. 16. In fact, with a $\sim 25^{\circ}$ tilt of the benzene rings the two perpendicular transition moments in the plane of the ring would be expected to make roughly about the same angle with the helix axis. On the basis of these results we are led to favor the structure shown in Fig. 16. (Recent x-ray diffraction studies [NATTA, CoRradiNI, and BASSI (154b)] support this conclusion.)

The analysis of the spectrum of isotactic polystyrene is in many respects similar to that of isotactic polypropylene. Both chain structures 
have the same symmetry, and if we restrict ourselves again to a single chain analysis (as seems to be valid here), the modes of the polystyrene chain should split into $A$ and $E$ species. The spectrum of isotactic polystyrene, when compared with that of the atactic polymer, however, indicates that in general this splitting is not observed. The analysis can therefore be carried out largely in terms of that of the atactic polymer [LIANG and KRIMM (174)], except, as we shall see, for those ring modes which do seem to split up into $A$ and $E$ species in the chain modes. As was pointed out earlier (section III. A. 1.), the modes of the benzene ring can be satisfactorily analyzed in terms of the local symmetry of the ring, which for monosubstituted benzene is $\mathrm{C}_{2 v}$. Symmetry coordinates for the ring modes have been suggested [LIANG and KRIMM (114)], and are shown in Fig. 17. The $A_{1}, B_{1}$, and $B_{2}$ modes are predicted to be infrared active, while all of the species give rise to Raman active modes. The remaining modes are those of the $\mathrm{CH}$ and $\mathrm{CH}_{2}$ groups and the carbon chain.

The analysis can be carried out fairly satisfactorily on the basis of the previous analysis of atactic polystyrene and the recent polarization and deuteration studies on isotactic polystyrene. Only a few modifications of the earlier assignments seem to be required. If we consider the $B_{2}$ ring modes first, these should be identifiable as $\pi$ bands in the spectrum. It will be observed that not only can they be assigned in accordance with the previous analysis [LIANG and KRIMM (174)], but these modes seem to split into $A(\pi)$ and $E(\sigma)$ components. This is true of the bands at 700 , 760 , and $906 \mathrm{~cm}^{-1}$, and apparently also for the components at 550 and $567 \mathrm{~cm}^{-1}$ [TAKedA, Imura, Yamada, and Imamura (222a)] (which seem to be correlated with the $542 \mathrm{~cm}^{-1}$ band of atactic polystyrene). The only questionable case is that of $\boldsymbol{\nu}_{5}\left(B_{2}\right):$ no $\pi$ band is observed in the expected frequency region $\left(\sim 990 \mathrm{~cm}^{-1}\right)$. It may be that this mode is too weak to be observed (it is inactive in the spectrum of benzene), or that it is to be assigned to the weak $\pi$ band at $1005 \mathrm{~cm}^{-1}$ observed in polystyrene [Tadokoro, Nozakura, Kitazawa, Yasuhara, and Murahashi (222)] and poly- $\alpha, \beta, \beta$-trideuterostyrene [Tadokoro, Nishiyama, Nozakura, and Murahashi (221)]. If these assignments are correct, and, as will be seen, the other modes do not exhibit the same degree of splitting, then we may infer that only for the out-of-plane ring vibrations is the interaction between monomer units along the chain sufficient to observably separate the $A$ and $E$ chain frequencies.

The remaining ring modes can be assigned on the basis of the previous analysis of atactic polystyrene. Slightly different assignments of the $\nu_{2}^{\prime}\left(A_{1}\right)$ and $v_{20 A}^{\prime}\left(A_{1}\right)$ modes are suggested here, although the evidence for any specific assignments in the hydrogen stretching.region is meager. The $\nu_{3}^{\prime}\left(B_{1}\right)$ and $\nu_{14}^{\prime}\left(B_{1}\right)$ modes are reassigned as a result of the change in 
$A_{f}:$

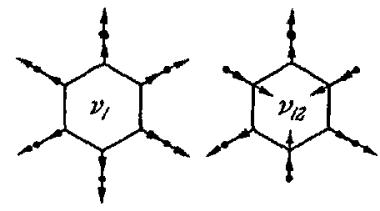

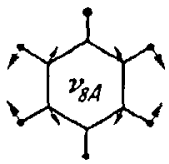

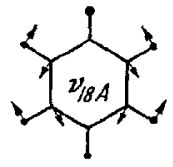

$B_{1}:$<smiles>CC1C(C)C2(C)CC1(C)C(C)C2C</smiles><smiles>CC1=C(C)C2(C)C(I)C(C)(C1C)C2(C)C</smiles><smiles>CC1=C(C)C(C)C(C)=C(C)C1C</smiles><smiles>CCC1=C(C)C(C)(CC)C(C)=C(CC)C1CC</smiles><smiles>CC1C(C)C(C)C(C)C(C)C1C</smiles>

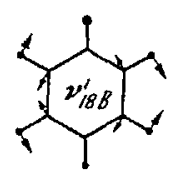<smiles>[CH]C1=C(C)C2(C)C(C)C(C)C1(C)C(C)C2(C)C</smiles><smiles>C=C1C2(C)CC(C)=C(C)C(C)(C2)C1(C)C</smiles>
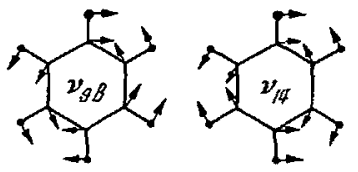

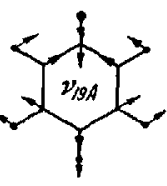<smiles>CC1=C(C)C2(C)CC1(C)C(C)C2(C)C</smiles><smiles>CC1C(C)(C)C2(C)CCC1(C)C2(C)C</smiles>

$A_{Z}:$

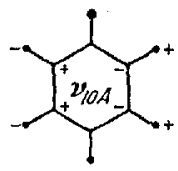<smiles>CC1=C(C)C2(C)C(C)C(C)C1(C)C2C</smiles>

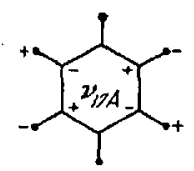

$B_{2}$ :<smiles>CC1=C(C)C(C)C(C)=C(C)C1C</smiles><smiles>CC1=C(C)C(C)C(C)=C(C)C1C</smiles><smiles>CC1=C2C(C)=C(C)C(C)(C(C)=C1I)C2(C)C</smiles><smiles>[3H]c1c(C)c(C)c(C)c(C)c1C</smiles><smiles>[3H]C1=C(C)C2(C)C(C)=C(C)C1(C)C2(C)C</smiles><smiles>CC1=C(C)C2(C)C(C)=C(C)C1(C)C2(C)C</smiles>

Fig. 17. Approximate normal modes of monosubstituted benzene [LiANG and KR1MM (114)]

assignment of the $1298 \mathrm{~cm}^{-1}$ band; the new assignments seem satisfactory. Polarization and deuteration studies support the earlier assignments of the remaining ring modes. The changes which occur in the spectrum of the isotactic polymer seem to be associated primarily with changes in the relative intensities of some of the $\mathrm{CH}$ and $\mathrm{CH}_{2}$ modes, and the probable appearance of bands (e.g., at 1048 and $1182 \mathrm{~cm}^{-1}$ ) asso- 


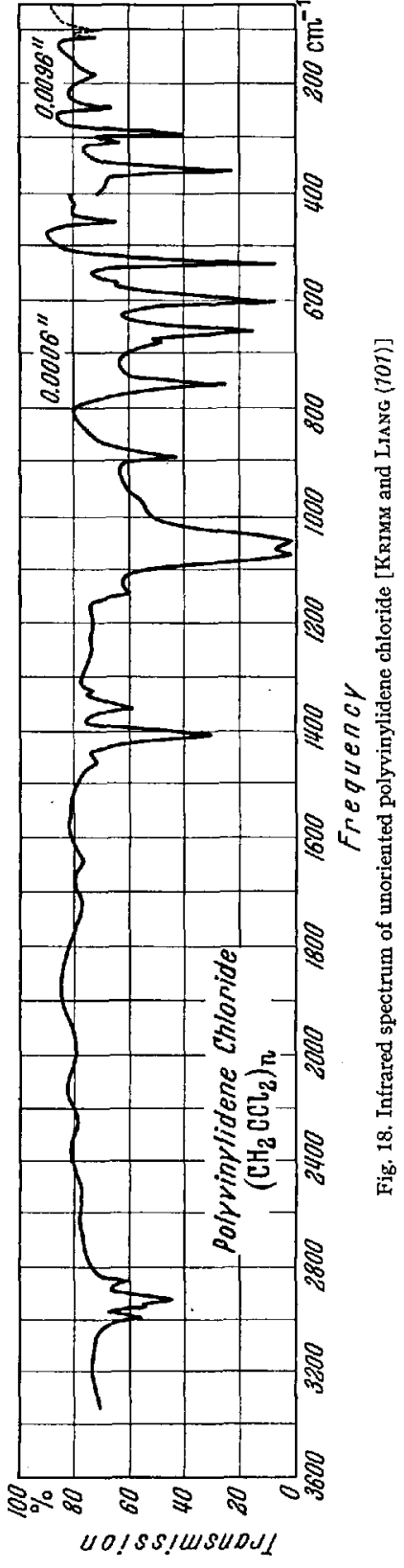

ciated with skeletal vibrations of the helical backbone. It will be noted that the $A_{1}, B_{1}$, and $A_{2}$ vibrations of the benzene ring do not exhibit splitting into $A$ and $E$ species chain vibrations. This emphasizes the earlier remark that apparently only for the out-ofplane ring modes does the intra-chain interaction lead to significant splitting between these species.

Several changes in assignments of the $\mathrm{CH}$ and $\mathrm{CH}_{2}$ modes are suggested by the recent polarization and deuteration studies. The assignments for $v_{s}\left(\mathrm{CH}_{2}\right)$ and $v_{a}\left(\mathrm{CH}_{2}\right)$ are unchanged. Their polarization, especially in $\left.{ }_{-}^{\prime} \mathrm{CD}_{2} \mathrm{CD}\right)_{n}^{\prime}$, where $v_{s}\left(\mathrm{CD}_{2}\right)$ is $\pi$ $\mathrm{C}_{6} \mathrm{H}_{5}$

and $\nu_{a}\left(\mathrm{CD}_{2}\right)$ is $\sigma$, provides further spectroscopic support for the proposed helical structure (see Table 15). In isotactic polystyrene a shoulder is observed at $1440 \mathrm{~cm}^{-1}$ which disappears on deuteration: this is undoubtedly the $\delta\left(\mathrm{CH}_{2}\right)$ mode. As was suggested by the earlier analysis and is confirmed by the deuteration results, the $1376 \mathrm{~cm}^{-1}$ band (at $1364 \mathrm{~cm}^{-1}$ in the isotactic polymer) seems to be associated with a $\delta(\mathrm{CH})$ mode. The $1310 \mathrm{~cm}^{-1}$ band of atactic polystyrene appears to be split in the isotactic polymer, with components at $1297 \mathrm{~cm}^{-1}(\pi)$ and $1314 \mathrm{~cm}^{-1}(\sigma)$. If these bands in fact go together, then an assignment to the $A$ and $E$ species $\gamma_{t}\left(\mathrm{CH}_{2}\right)$ modes is reasonable. The other $\mathrm{CH}$ and $\mathrm{CH}_{2}$ modes do not seem to be identifiable, except perhaps $\gamma_{r}\left(\mathrm{CH}_{2}\right)$, and this may be a result of their weakness or overlap by benzene ring vibrations.

Although some aspects of the proposed assignments will require additional confirmation in order to be more certain that they are correct, it appears at present that the basic framework of a set of assignments for isotactic polystyrene exists. These assignments enable the use of the infrared spectrum of an oriented 
specimen to determine the orientation of the benzene ring on the helix. They also suggest the nature of some of the interactions within the chain.

\section{G. Polyvinylidene Chloride}

The spectrum of polyvinylidene chloride $\left.-{ }_{-}^{\prime} \mathrm{CH}_{2} \mathrm{CCl}_{2}\right)_{n},(\mathrm{PVdC})$ provides another illustration of the possibility of using the infrared spectrum as a means of obtaining information on the chain configuration. In this case,

Table 17. Infrared Spectrum of Polyvinylidene Chloride

\begin{tabular}{|c|c|c|c|}
\hline $\begin{array}{l}\text { Frequency, } \\
\mathrm{cm}^{-1}\end{array}$ & R.I. & Pol'n & Assignment \\
\hline 102 & $\mathrm{vw}$ & & Skeletal? \\
\hline 113 & $\mathrm{vw}$ & & Skeletal? \\
\hline 185 & $\mathrm{w}$ & & Skeletal? \\
\hline 245 & $\mathrm{w}$ & & $\delta\left(\mathrm{CCl}_{2}\right)_{i}\left(\mathrm{~A}^{\prime}\right)$ \\
\hline 291 & In & & $\gamma_{r}\left(\mathrm{CCl}_{2}\right)_{i}\left(\mathrm{~A}^{\prime}\right)$ \\
\hline 307 & $\mathrm{w}$ & & $\gamma_{r}\left(\mathrm{CCl}_{2}\right)_{0}\left(\mathrm{~A}^{\prime}\right)$ \\
\hline 359 & $\mathrm{~m}$ & $\pi$ & $\gamma_{w}\left(\mathrm{CCl}_{2}{ }_{i}\left(\mathrm{~A}^{\prime \prime}\right)\right.$ \\
\hline 430 & vw & $\pi$ & $\gamma_{l}\left(\mathrm{CCl}_{2}\right)_{0}\left(\mathrm{~A}^{\prime \prime}\right) ? ;$ Skeletal? \\
\hline 454 & $\mathrm{~m}$ & $\pi$ & $\gamma_{t}\left(\mathrm{CCl}_{2}\right)_{i}\left(\mathrm{~A}^{\prime \prime}\right)$ ?; Skeletal? \\
\hline 530 & vs & $\sigma$ & $v_{s}\left(\mathrm{CCl}_{2}\right)_{i}\left(\mathrm{~A}^{\prime}\right)$ \\
\hline 565 & $\mathrm{vw}$ & & \\
\hline 603 & vs & $\sigma$ & $v_{s}\left(\mathrm{CCl}_{2}\right)_{0}\left(\mathrm{~A}^{\prime}\right)$ \\
\hline 657 & $\mathrm{~s}$ & $\sigma$ & $v_{a}\left(\mathrm{CCl}_{2}\right)_{i}\left(\mathrm{~A}^{\prime}\right)$ \\
\hline 754 & s & $\sigma$ & $\nu_{a}\left(\mathrm{CCl}_{2}\right)_{0}\left(\mathrm{~A}^{\prime}\right)$ \\
\hline 778 & vw & $\sigma$ & $\gamma_{r}\left(\mathrm{CH}_{2}\right)_{i}\left(\mathrm{~A}^{\prime}\right)$ \\
\hline 887 & $\mathrm{~m}$ & $\sigma$ & $\nu_{+}\left(\frac{3 \pi}{2}\right) ?$ \\
\hline 980 & w & $\sigma$ & $v_{a}\left(\mathrm{CCl}_{2}\right)_{0}+\delta\left(\mathrm{CCl}_{2}\right)_{i}=999 ?$ \\
\hline 1046 & vs & $\sigma)$ & $v_{+}(0) ; 2 \times v_{s}\left(\mathrm{CCl}_{2}\right)_{i}=1060$ \\
\hline 1070 & vs & $\sigma]$ & \\
\hline 1142 & m & $\pi$ & $\nu_{+}(\pi)$ \\
\hline 1204 & vvw & & $2 \times v_{s}\left(\mathrm{CCl}_{2}\right)_{0}=1206$ \\
\hline 1290 & vow & $\pi$ ? & $\gamma_{t}\left(\mathrm{CH}_{2}\right)_{i}\left(\mathrm{~A}^{\prime \prime}\right)$ \\
\hline 1325 & $\mathbf{w}$ & $\sigma$ & $\gamma_{w}\left(\mathrm{CH}_{2}\right)_{0}\left(\mathrm{~A}^{\prime}\right)$ \\
\hline 1360 & $\mathrm{~m}$ & $\pi$ & $\gamma_{10}\left(\mathrm{CH}_{2}\right)_{i}\left(\mathrm{~A}^{\prime \prime}\right)$ \\
\hline 1407 & $\mathrm{~s}$ & $\sigma$ & $\delta\left(\mathrm{CH}_{2}\right)_{t}\left(\mathrm{~A}^{\prime}\right)$ \\
\hline 1460 & $\mathrm{w}$ & $\sigma$ & $\delta\left(\mathrm{CH}_{2}\right)$ (amorphous) ? \\
\hline 2850 & $\mathrm{w}$ & $\sigma ?$ & $v_{3}\left(\mathrm{CH}_{2}\right)$ (amorphous)? \\
\hline 2930 & $\mathrm{~m}$ & $\sigma$ & $v_{a}\left(\mathrm{CH}_{2}\right)$ (amorphous) ? \\
\hline 2948 & vw & $\sigma ?$ & $v_{s}\left(\mathrm{CH}_{2}\right)_{i}\left(\mathrm{~A}^{\prime}\right)$ \\
\hline 2990 & $\mathrm{~m}$ & $\sigma$ & $v_{a}\left(\mathrm{CH}_{2}\right)_{i}\left(\mathrm{~A}^{\prime}\right)$ \\
\hline
\end{tabular}

two different chain structures have been proposed on the basis of $\mathrm{x}$-ray diffraction studies. The infrared spectrum of oriented PVdC samples provides a means of deciding between these two structures if a correct interpretation of the spectrum can be achieved. As we shall see, several important questions arise in connection with the latter problem.

The infrared spectrum of PVdC is shown in Fig. 18 [KRIMM and LIANG (101)]. It is not possible to orient a PVdC film in order to obtain polarized spectra, but when a copolymer is made of vinylidene chloride 
with a small amount of vinyl chloride the resulting specimen can be oriented. Since the contribution of the vinylidene chloride predominates (only bands at 868 and $1206 \mathrm{~cm}^{-1}$ are specific to the copolymer [NARITA, ICHINOHE, and ENOMOTo (146)]), it is possible to identify the PVdC bands and determine their polarization [KRIMM and Liang (101); NARITA, ICHINOHE, and ENomoto (147)]. These, together with band positions are given in Table 17.

The unique aspect of the structure of PVdC is exhibited by its chain axis identity period of about $4.7 \mathrm{~A}$ [Fuller (64); NARITA and OKUDA (143)]. This indicates that there is more than one monomer unit in the

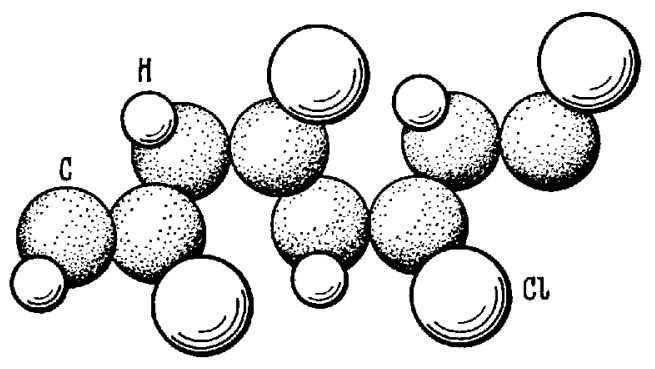

a)

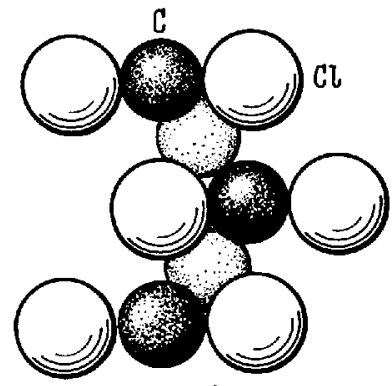

b)

Fig. 19a and b. Proposed structures of polyvinylidene chloride. a) ReInHardr (785). b) Fuller (64)

chain repeat, in all probability two. Yet, if there are two monomer units per repeat, the chain must be in a configuration other than the planar zig-zag, since this would give rise to a fiber axis repeat of $5.1 \mathrm{~A}$. Two models have been proposed for the manner in which the carbon skeleton folds in order to give the observed shorter repeat distance. One, due to REINHARDT (185), is shown in Fig. 19a; the other, due to Fuller (64), is shown in Fig. 19b. As has been shown [KRIMM and Liang (101)], these structures predict different numbers and polarizations of bands associated with the $\nu\left(\mathrm{CCl}_{2}\right)$ modes. It was also shown that the assignment of the four strong bands at $530,603,657$, and $754 \mathrm{~cm}^{-1}$ to $v\left(\mathrm{CCl}_{2}\right)$ modes, which is indicated, favored the Fuller structure as the correct one. Recent work on deuterated PVdC [NARITA, Ichinohe, and Enomoto (148)] has questioned the above interpretation, and it is therefore appropriate to re-examine the spectral assignments in detail.

The assignment of the above-mentioned four bands can be clarified by reference to the spectra of deuterated $\mathrm{PVdC}$ and polyvinylidene bromide [NARITA, ICHINOHE, and ENomoto (147)]. The latter crystallizes in a unit cell very similar in size to that of $\mathrm{PVdC}$, its fiber axis repeat being $4.77 \mathrm{~A}$ rather than the $4.68 \mathrm{~A}$ of PVdC [NARITA and OKUDA (143)]. We may therefore assume that the two structures are essentially similar. In the 
appropriate region, PVdB exhibits bands at 416 (v), 518 (vs), 599 (s), and $717(m) \mathrm{cm}^{-1}$. From the $v\left(\mathrm{CX}_{2}\right)$ bands in the spectra of $\mathrm{CH}_{3} \mathrm{CCl}_{2} \mathrm{CH}_{3}$ and $\mathrm{CH}_{3} \mathrm{CBr}_{2} \mathrm{CH}_{3}$ [KoHLRAUSCH (92)], viz., at 557 and $652 \mathrm{~cm}^{-1}$ in the former case and 481 and $585 \mathrm{~cm}^{-1}$ in the latter, it can be inferred that the 657 and $603 \mathrm{~cm}^{-1}$ bands of $\mathrm{PVdC}$ shift to 599 and $518 \mathrm{~cm}^{-1}$ respectively in PVdB. This implies the further correspondences of the 754 and $530 \mathrm{~cm}^{-1}$ bands of PVdC with the 717 and $416 \mathrm{~cm}^{-1}$ bands of PVdB. (The weak intensity of the $416 \mathrm{~cm}^{-1}$ band in comparison with that at $530 \mathrm{~cm}^{-1}$ may be a result of the slight variation in identity period and therefore structure of PVdB as compared with PVdC.) The essentially similar features of the rest of the PVdB spectrum are thus seen to carry through to the $\nu\left(\mathrm{CX}_{2}\right)$ region. The primary question to be answered in connection with the assignments is whether the $\mathbf{7 5 4} \mathrm{cm}^{-1}$ band of $\mathrm{PVdC}$ is a $v\left(\mathrm{CCl}_{2}\right)$ mode, as suggested earlier [KRIMM and LIANG (701)], or a $\gamma_{r}\left(\mathrm{CH}_{2}\right)$ mode as has been suggested recently [NARITA, ICHINOHE, and Enomoto (148)]. Its shift to $717 \mathrm{~cm}^{-1}$ in PVdB, a rather low value for a $\gamma_{r}\left(\mathrm{CH}_{2}\right)$ mode, suggests that such an assignment must be carefully questioned. The value of $754 \mathrm{~cm}^{-1}$, on the other hand, is not too high for a $\nu\left(\mathrm{CCl}_{2}\right)$ mode: in $\mathrm{CHCl}_{2} \mathrm{CHCl}_{2}$ such modes are found as high as $814 \mathrm{~cm}^{-1}$ [NAITO, NAKAGaWA, Kuratani, ICHishima, and Mizushima (142)].

Two other principal lines of evidence indicate that the $754 \mathrm{~cm}^{-1}$ band should be assigned to a $\nu\left(\mathrm{CCl}_{2}\right)$ mode. First, the shifts of known $\mathrm{CH}_{2}$ modes between PVdC and PVdB is much less than that of the $754 \mathrm{~cm}^{-1}$ band. For example, $\delta\left(\mathrm{CH}_{2}\right)$ shifts from $1407 \mathrm{~cm}^{-1}$ in $\mathrm{PVdC}$ to $1396 \mathrm{~cm}^{-1}$ in $\mathrm{PVdB}$, a ratio of 1.007 , and $\gamma_{w}\left(\mathrm{CH}_{2}\right)$ shifts from 1357 to $1347 \mathrm{~cm}^{-1}$, a ratio of 1.007 , whereas the shift in the $754 \mathrm{~cm}^{-1}$ band corresponds to a ratio of 1.050. This is markedly out of line. The band in $\mathrm{PVdC}$ which we will later suggest should be assigned to $\gamma_{r}\left(\mathrm{CH}_{2}\right)$, viz., the weak band at about $778 \mathrm{~cm}^{-1}$, has a counterpart at $773 \mathrm{~cm}^{-1}$ in the PVdB spectrum. The shift ratio is 1.007, in excellent agreement with that of the other $\mathrm{CH}_{2}$ modes. Second, the band shifts on deuteration agree quite well with the predicted values for all but the $754 \mathrm{~cm}^{-1}$ band, which suggests that it is not associated with a $\mathrm{CH}_{2}$ mode. Thus, from the results of deuteration studies [NARITA, ICHINOHE, and ENomoto (747)] we find the following frequency shift ratios: $v_{a}\left(\mathrm{CH}_{2}\right)-1.340, v_{s}\left(\mathrm{CH}_{2}\right)-1.378, \delta\left(\mathrm{CH}_{2}\right)-1.340$ $\gamma_{w}\left(\mathrm{CH}_{2}\right)-1.322$. The excellent agreement with the predicted values (see Table 4) suggests that for $\gamma_{r}\left(\mathrm{CH}_{2}\right)$ a frequency shift of about 1.38 should be expected. Yet the shift for the recently suggested assignment is $754 / 607=1.24$, completely out of line. If the $754 \mathrm{~cm}^{-1}$ band were to shift by about 1.38 , we would expect the associated $\gamma_{r}\left(\mathrm{CD}_{2}\right)$ mode to occur at about $545 \mathrm{~cm}^{-1}$ : no band appears near this position in deuterated PVdC. The assignment of the $778 \mathrm{~cm}^{-1}$ band to $\gamma_{r}\left(\mathrm{CH}_{2}\right)$, however, results in the prediction of the $\gamma_{r}\left(\mathrm{CD}_{2}\right)$ mode at about $564 \mathrm{~cm}^{-1}$ : a comparably weak 
band is found at $560 \mathrm{~cm}^{-1}$ in deuterated PVdC. The evidence therefore strongly favors the assignment of the $754 \mathrm{~cm}^{-1}$ band to a $v\left(\mathrm{CCl}_{2}\right)$ mode. Thus, on the basis of this more conclusive evidence for the existence of four $\sigma v\left(\mathrm{CCl}_{2}\right)$ modes in $\mathrm{PVdC}$ it seems valid to maintain the conclusions leading to the acceptance of the Fuller structure. These arguments [KRIMM and LIANG (101)] would lose some of their force if it should be shown that not all of these four bands are associated with the crystalline material, but, for example, some are due to rotational isomers in the amorphous regions, as is the case for PVC. There is not sufficient evidence

Table 18. Classification of $\mathrm{CH}_{2}$ and $\mathrm{CCl}_{2}$ Modes in Polyvinylidene Chloride

\begin{tabular}{|c|c|c|c|c|c|}
\hline \multicolumn{3}{|c|}{$\mathrm{CH}_{3}$} & \multicolumn{3}{|c|}{$\mathrm{CCI}_{2}$} \\
\hline Mode & Pol'n & R. I. ${ }^{1}$ & Mode & Pol'n & R. I. ${ }^{1}$ \\
\hline$\nu_{s}\left(\mathrm{CH}_{2}\right)_{i}\left(\mathrm{~A}^{\prime}\right)$ & $\sigma$ & $s$ & $v_{x}\left(\mathrm{CCl}_{2}\right)_{s}\left(\mathrm{~A}^{\prime}\right)$ & $\sigma$ & $\mathbf{s}$ \\
\hline$\nu_{s}\left(\mathrm{CH}_{2}\right)_{0}\left(\mathrm{~A}^{\prime \prime}\right)$ & $\pi$ & $\mathbf{w}$ & $v_{s}\left(\mathrm{CCl}_{2}\right)_{0}\left(\mathrm{~A}^{\prime}\right)$ & $\sigma$ & $\mathrm{w}$ \\
\hline$v_{a}\left(\mathrm{CH}_{2}\right)_{i}\left(\mathrm{~A}^{\prime}\right)$ & $\sigma$ & $\mathrm{s}$ & $v_{a}\left(\mathrm{CCl}_{2}\right)_{i}\left(\mathrm{~A}^{\prime}\right)$ & $\sigma$ & $\mathbf{s}$ \\
\hline$\nu_{a}\left(\mathrm{CH}_{2}\right)_{0}\left(\mathrm{~A}^{\prime \prime}\right)$ & $\pi$ & $\mathrm{w}$ & $v_{a}\left(\mathrm{CCl}_{2}\right)_{0}\left(\mathrm{~A}^{\prime}\right)$ & $\sigma$ & $\mathrm{w}$ \\
\hline$\delta\left(\mathrm{CH}_{2}\right)_{i}\left(\mathrm{~A}^{\prime}\right)$ & $\sigma$ & $s$ & $\delta\left(\mathrm{CCl}_{2}\right)_{i}\left(\mathrm{~A}^{\prime}\right)$ & $\sigma$ & $\mathrm{s}$ \\
\hline$\delta\left(\mathrm{CH}_{2}\right)_{0}\left(\mathrm{~A}^{\prime \prime}\right)$ & $\pi$ & $\mathrm{w}$ & $\delta\left(\mathrm{CCl}_{2}\right)_{0}\left(\mathrm{~A}^{\prime}\right)$ & $\sigma$ & $\mathrm{w}$ \\
\hline$\gamma_{s o}\left(\mathrm{CH}_{2}\right)_{0}\left(\mathrm{~A}^{\prime}\right)$ & $\sigma$ & $\mathrm{w}$ & $\gamma_{* 0}\left(\mathrm{CCl}_{2}\right)_{i}\left(\mathrm{~A}^{\prime \prime}\right)$ & $\pi$ & $\mathrm{s}$ \\
\hline$\gamma_{w}\left(\mathrm{CH}_{2}\right)_{i}\left(\mathrm{~A}^{\prime \prime}\right)$ & $\pi$ & $\mathrm{s}$ & $\gamma_{w}\left(\mathrm{CCl}_{2}\right)_{0}\left(\mathrm{~A}^{\prime \prime}\right)$ & $\pi$ & $w$ \\
\hline$\gamma_{r}\left(\mathrm{CH}_{2}\right)_{i}\left(\mathrm{~A}^{\prime}\right)$ & $\sigma$ & $\mathrm{s}$ & $\gamma_{r}\left(\mathrm{CCl}_{2}\right)_{i}\left(\mathrm{~A}^{\prime}\right)$ & $\sigma$ & $\mathbf{s}$ \\
\hline$\gamma_{r}\left(\mathrm{CH}_{2}\right)_{0}\left(\mathrm{~A}^{\prime \prime}\right)$ & $\pi$ & $\mathrm{w}$ & $\gamma_{r}\left(\mathrm{CCl}_{2}\right)_{0}\left(\mathrm{~A}^{\prime}\right)$ & $\sigma$ & $\mathrm{w}$ \\
\hline$\gamma_{l}\left(\mathrm{CH}_{2}\right)_{0}\left(\mathrm{~A}^{\prime}\right)$ & $\sigma$ & $\mathrm{w}$ & $\gamma_{t}\left(\mathrm{CCl}_{2}\right)_{i}\left(\mathrm{~A}^{\prime \prime}\right)$ & $\pi$ & $s$ \\
\hline$\gamma_{l}\left(\mathrm{CH}_{2}\right)_{i}\left(\mathrm{~A}^{\prime \prime}\right)$ & $\pi$ & $\mathrm{s}$ & $\gamma_{t}\left(\mathrm{CCl}_{2}\right)_{0}\left(\mathrm{~A}^{\prime \prime}\right)$ & $\pi$ & $w$ \\
\hline
\end{tabular}

1 The designations $s$ and $w$ refer only to a comparison of the intensities of the $i$ and $o$ modes for a given vibration.

on this point from the study of small molecules, but the spectra of melted specimens of PVdC [NARITA, IchINoHe, and Enomoto (147)] suggest that this is not the case: all four bands diminish about equally in intensity on melting. We will therefore assume the Fuller structure as the basis for a spectral analysis of PVdC.

As has been shown [Krimm and Liang (101)], the Fuller structure for PVdC has only one symmetry element, a mirror plane through the $\mathrm{CCl}_{2}$ groups, and this leads to a division of modes into those symmetric with respect to the plane $\left(A^{\prime}, \sigma\right)$ and those antisymmetric with respect to this plane $\left(A^{\prime \prime}, \pi\right)$. These can be further subdivided (in terms of the group mode approximation) into $12 \mathrm{CH}_{2}$ vibrations $\left(6 A^{\prime}+6 A^{\prime \prime}\right), 12 \mathrm{CCl}_{2}$ vibrations $\left(8 A^{\prime}+4 A^{\prime \prime}\right), 8$ carbon chain vibrations $\left(4 A^{\prime}+4 A^{\prime \prime}\right)$, one rotation about the chain axis $\left(A^{\prime}\right)$, and 3 translations $\left(2 A^{\prime}+1 A^{\prime \prime}\right)$. The relative intensities of some of these modes can be predicted from the structure [KRIMM and LIANG (101)]; this information, shown in Table 18 for the $\mathrm{CH}_{2}$ and $\mathrm{CCl}_{2}$ modes, is of help in making the assignments. As in the PVC case, we will designate by a subscript $i$ or o modes in which the 
motions in the two monomer units comprising the fiber axis repeat are, respectively, in-phase or out-of-phase with each other. Some corrections of the earlier results are included in Table 18.

The assignments of the $\nu\left(\mathrm{CH}_{2}\right)$ bands in terms of the assumed model are not entirely unambiguous. Two strong $\sigma$ bands and two weak $\pi$ bands $\left[v_{s}\left(\mathrm{CH}_{2}\right)_{0}\right.$ is probably close to zero intensity] are predicted. In pure PVdC four bands are found, all apparently of $\sigma$ polarization. Two of these, however, at 2850 and $2930 \mathrm{~cm}^{-1}$, are of highly variable intensity; in the copolymer spectra they become quite weak in comparison to the other two at 2948 and $2990 \mathrm{~cm}^{-1}$ [NARITA, IcHINOHE, and ENomoto (147) quote frequency values of 2966 and $3010 \mathrm{~cm}^{-1}$ for these two bands]. A possible explanation is that the 2850 and $2930 \mathrm{~cm}^{-1}$ bands are associated with amorphous structures, while the 2948 and $2990 \mathrm{~cm}^{-1}$ bands are associated with the Fuller structure. We would then have to assume that the $v_{a}\left(\mathrm{CH}_{2}\right)_{0}$ mode is too weak to be observed. It is clear that these proposals require additional confirmation.

The other $\mathrm{CH}_{2}$ modes are more readily identified. The band associated with $\delta\left(\mathrm{CH}_{2}\right)_{i}$ is observed at $1407 \mathrm{~cm}^{-1}$. The $\delta\left(\mathrm{CH}_{2}\right)_{0}$ mode, which should give rise to a $\pi$ band, is expected to have nearly zero intensity. The $1460 \mathrm{~cm}^{-1}$ band may be a $\delta\left(\mathrm{CH}_{2}\right)$ mode associated with amorphous chains since it also exhibits variable intensity. The $1360 \mathrm{~cm}^{-1} \pi$ band is most likely to be $\gamma_{w}\left(\mathrm{CH}_{2}\right)_{i}$, and the weak $\sigma$ band at $1325 \mathrm{~cm}^{-1}$ may be $\gamma_{w}\left(\mathrm{CH}_{2}\right)_{0}$. As we have noted earlier, the reasons for assigning $\gamma_{r}\left(\mathrm{CH}_{2}\right)_{i}$ to the weak band at about $778 \mathrm{~cm}^{-1}$ are more compelling than any other assignment. The $\gamma_{r}\left(\mathrm{CH}_{2}\right)_{0}$ mode seems not to be observable. The $\gamma_{t}\left(\mathrm{CH}_{2}\right)_{i}$ mode may be the origin of the very weak band at about $1290 \mathrm{~cm}^{-1}$.

Of the chlorine vibrations, the $\nu\left(\mathrm{CCl}_{2}\right)$ modes are, as we have seen, satisfactorily assigned to the four strong $\sigma$ bands in the $500-800 \mathrm{~cm}^{-1}$ region. The exact assignments are uncertain, but we may tentatively correlate the lower frequencies with the $\nu_{s}\left(\mathrm{CCl}_{2}\right)$ vibrations. The $\delta\left(\mathrm{CCl}_{2}\right)$, $\gamma_{r}\left(\mathrm{CCl}_{2}\right)$, and $\gamma_{w}\left(\mathrm{CCl}_{2}\right)$ modes cannot be identified with certainty, but arguments based on the spectrum of $\mathrm{CH}_{3} \mathrm{CCl}_{2} \mathrm{CH}_{3}$ [KRIMM and LIANG (101)] appear to lead to fairly satisfactory assignments. The $\gamma_{t}\left(\mathrm{CCl}_{2}\right)$ mode has not been identified in the spectra of small molecules, and we can only hazard the suggestion that it may be contributing to the $\pi$ bands at 430 and $454 \mathrm{~cm}^{-1}$. Of course, the distinction between $\mathrm{CCl}_{2}$ and carbon chain skeletal vibrations may not be completely valid, and the above designations for the $\mathrm{CCl}_{2}$ modes should be considered in this light. Several of the remaining bands in the spectrum can be assigned to skeletal vibrations, although the exact nature of these modes is presently unknown. Some of them probably resemble the modes of a planar zig-zag chain; the form of the others has not yet been determined. 


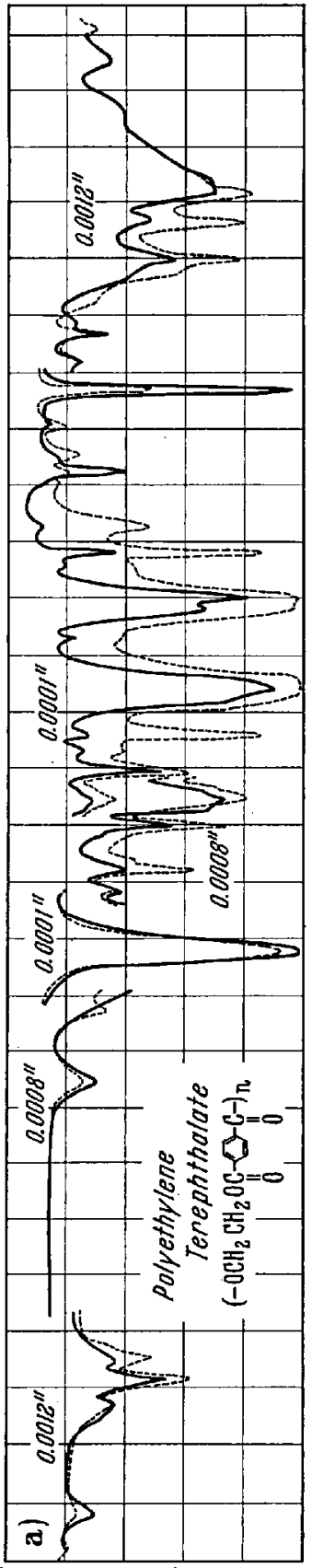

คำร
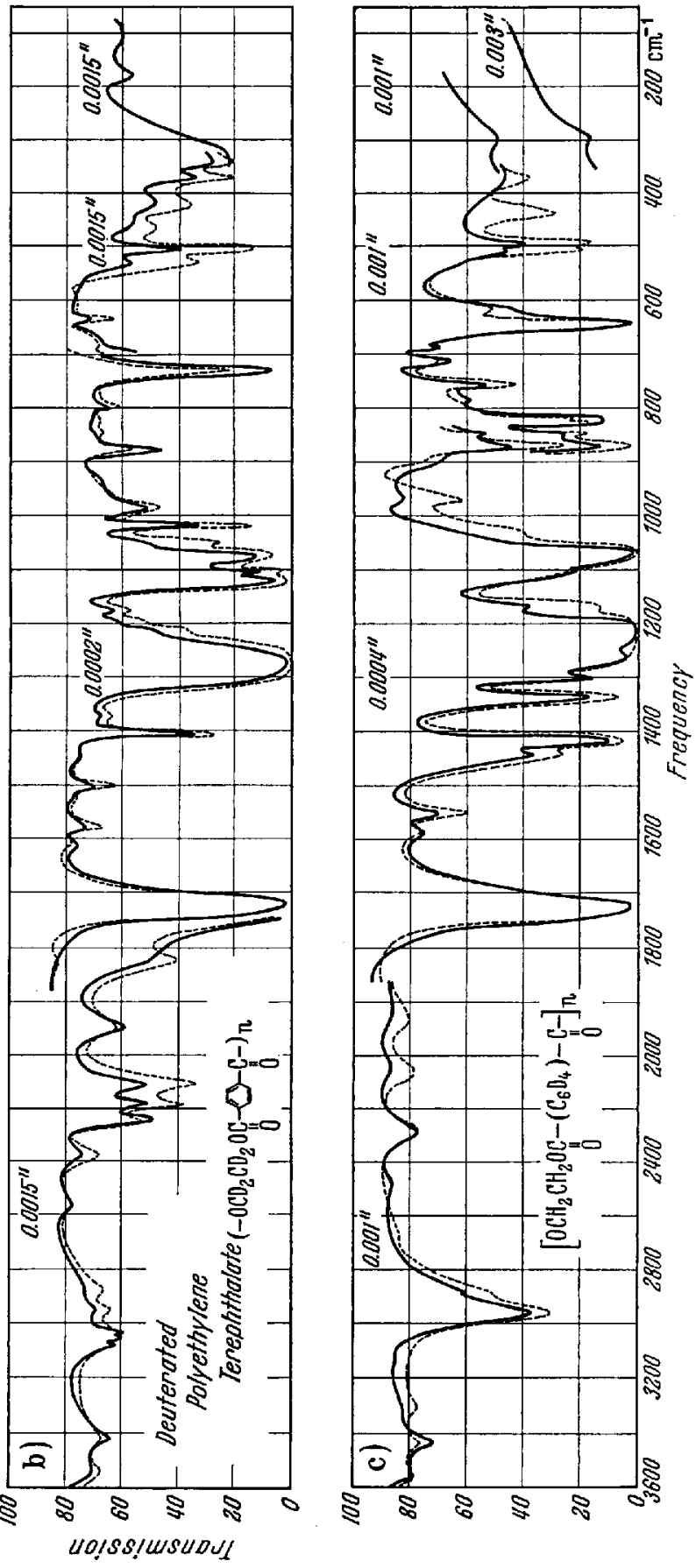


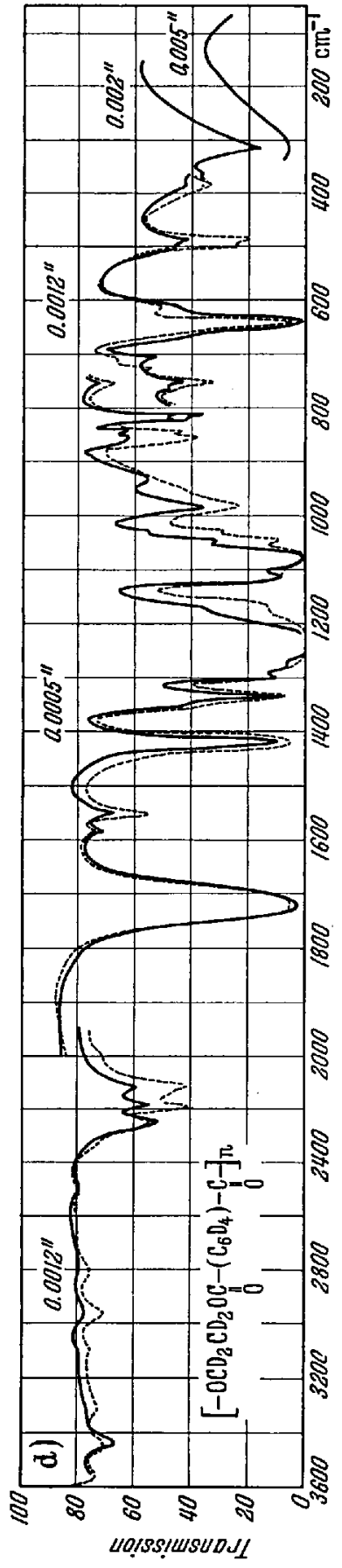

While it cannot be said that all of the assignments in the spectrum of $\mathrm{PVdC}$ are satisfactorily established, it would seem that the majority are. The important ones from the standpoint of the chain structure are the $\nu\left(\mathrm{CCl}_{2}\right)$ modes, and the present evidence on their assignment supports the correctness of the FuLLER structure. A study of a partially deuterated polymer might be of further assistance in this respect. What is also clearly needed is a more detailed study of the skeletal modes to be expected from the proposed chain structure, since these clearly contribute significantly to the spectrum. Finally, knowledge of the crystal structure might permit more to be said on the nature of interchain interactions and their effect on the spectrum.

\section{H. Polyethylene Terephthalate}

One of the most complex polymer molecules to be analyzed spectroscopically is polyethylene terephthalate, $\left.-\mathrm{OCH}_{2} \mathrm{CH}_{2} \mathrm{OOC}=\mathrm{CO}\right)_{\bar{n}}$, (PETP). As would be expected, the spectrum is quite complicated from the standpoint of the number of bands present. In addition, several other unusual features exist: there are marked intensity changes in some of the bands as a function of the crystallinity of the specimen, and several bands of fairly unambiguous origin exhibit dichroism which is the opposite of that expected from the proposed structure of the chain. An understanding of these aspects of the spectrum would lead directly to a more detailed knowledge of the crystalline molecular structure and the way in which it changes when a molecule enters the amorphous phase. As a result a great deal of work has been 
Table 19. Infrared Spectrum of Polyethylene Terephthatate

\begin{tabular}{|c|c|c|c|c|}
\hline $\begin{array}{c}\text { Frequency, } \\
\mathrm{cm}^{-1}\end{array}$ & R. I. & Pol'n & Phase & Assignment \\
\hline$\sim 95$ & vw & & & \\
\hline 145 & w & & & $\nu_{10 B}\left(\mathrm{~B}_{3 g}\right)$ \\
\hline 250 & $\mathrm{vw}$ & & & $\nu_{18 B}^{\prime}\left(\mathrm{B}_{\varepsilon u}\right)$ \\
\hline 355 & $\mathrm{w}$ & $\sigma$ & & $\gamma_{r}(\mathrm{C}=\mathrm{O})$ \\
\hline 383 & $\mathrm{~m}$ & $\pi$ & $\mathrm{C}$ & $\delta(\mathrm{COC})$ \\
\hline 430 & $w$ & $\sigma$ & & $v_{16 B}\left(\mathrm{~B}_{1 u}\right)$ \\
\hline 437 & $\mathrm{~m}$ & $\pi$ & $\mathrm{C}$ & $\delta(\mathrm{CCO})$ \\
\hline 502 & $\mathrm{~m}$ & $\pi$ & A & $\gamma_{w}(\mathrm{C}=\mathrm{O})$ \\
\hline 525 & $\mathrm{~m}$ & $\pi$ & A & $v_{6 A}\left(A_{1}\right)$ \\
\hline 575 & vw & $\pi$ & & $\nu_{10 B}\left(\mathrm{~B}_{3 \jmath}: 145\right)+\nu_{16 B}\left(\mathrm{~B}_{1 u}: 430\right)=575\left(\mathrm{~B}_{2 u}\right)$ \\
\hline 613 & vw & $\sigma$ & A & $v_{6 B}\left(\mathrm{~B}_{1}\right)$ ? \\
\hline 633 & $\mathrm{w}$ & $\pi$ & $\mathrm{C}$ & $v_{18 B}^{\prime}+\delta(\mathrm{COC})=633$ \\
\hline 680 & vw & $\sigma$ & A & $v_{4}\left(\mathrm{~B}_{2}\right) ?$ \\
\hline $\begin{array}{l}727 \\
733\}\end{array}$ & $\mathrm{s}$ & $\sigma$ & & $v_{11}\left(\mathrm{~B}_{1 u}\right)$ \\
\hline 793 & $\mathrm{w}$ & $\pi$ & A ? & $\gamma_{r}(\mathrm{C}=\mathrm{O})+\delta(\mathrm{CCO})=792$ \\
\hline 845 & $\mathrm{w}$ & $\pi$ & $\mathrm{C}$ & $\gamma_{\tau}\left(\mathrm{CH}_{2}\right)$ (crystalline) \\
\hline 875 & $\mathrm{~m}$ & $\sigma$ & $\mathrm{C}$ & $v_{17 B}\left(\mathrm{~B}_{1 u}\right)$ \\
\hline 897 & vw & $\pi ?$ & A & $\gamma_{r}\left(\mathrm{CH}_{2}\right)$ (amorphous) \\
\hline 973 & $\mathrm{~m}$ & $\pi$ & $\mathrm{C}$ & $\left\{\begin{array}{l}v_{12}\left(\mathrm{~B}_{2 u}\right) ? \\
\nu(\mathrm{O}-\mathrm{C}) ?\end{array}\right.$ \\
\hline 988 & vw & & $\mathrm{C}$ & \\
\hline 1019 & $\mathrm{~s}$ & $\pi$ & & $v_{18 A}\left(B_{2 u}\right)$ \\
\hline 1042 & $\mathrm{w}$ & $\sigma ?$ & A & $v(\mathrm{CC})$ ? \\
\hline 1100 & $\mathrm{~s}$ & $\pi$ & A & $\left\{\begin{array}{l}v_{15}^{\prime}\left(\mathrm{B}_{3 u}\right) \\
v(\mathrm{O}-\mathrm{C}) ?\end{array}\right.$ \\
\hline 1109 & vw & $\ldots$ & $\mathrm{C}$ & \\
\hline 1120 & $\mathrm{~s}$ & $\pi$ & $\mathrm{C}$ & $v(\mathrm{O}-\mathrm{C})$ \\
\hline 1172 & w & $\sigma ?$ & A & $\nu_{9 A}\left(A_{1}\right) ?$ \\
\hline 1245 & w & & & $\|$ \\
\hline 1263 & vs & $\pi$ & & $v(\mathrm{C}-\mathrm{O})$ \\
\hline 1280 & $w$ & & & $v_{14}\left(\mathrm{~B}_{3 u}\right) ?$ \\
\hline 1343 & $\mathrm{~ms}$ & $\pi$ & $\mathrm{C}$ & $\gamma_{w}\left(\mathrm{CH}_{2}\right)$ (crystalline) \\
\hline 1370 & $\mathrm{w}$ & $\sigma$ & A & $\gamma_{w}\left(\mathrm{CH}_{2}\right)$ (amorphous) \\
\hline 1387 & $w$ & & $\mathrm{C}$ & $y_{1 a B}\left(\mathrm{~B}_{3 u}\right) ?$ \\
\hline 1410 & s & $\pi$ & & 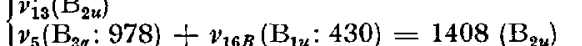 \\
\hline 1435 & vw & $\pi ?$ & & \\
\hline 1455 & $\mathrm{~m}$ & $\pi$ & & $\delta\left(\mathrm{CH}_{2}\right)$ (amorphous) \\
\hline 1473 & $w$ & $\sigma$ & C & $\delta\left(\mathrm{CH}_{2}\right)$ (crystalline) \\
\hline 1504 & $\mathrm{~m}$ & $\pi$ & & $v_{19 A}\left(\mathrm{~B}_{2 u}\right)$ \\
\hline 1525 & vw & - & & $v(\mathrm{O}-\mathrm{C})(1100)+\delta(\mathrm{CCO})=1537$ \\
\hline 1563 & vw & - & & $\nu(\mathrm{O}-\mathrm{C})(1120)+\delta(\mathrm{CCO})=1557$ \\
\hline 1580 & $\mathrm{mw}$ & $\pi$ & A & $\nu_{8 A}\left(A_{1}\right)$ \\
\hline 1617 & $\mathrm{w}$ & $\sigma$ & A & $\nu_{B B}\left(B_{1}\right)$ \\
\hline 1725 & vs & $\sigma$ & & $v(\mathrm{C}=\mathrm{O})$ \\
\hline 1830 & $\mathrm{w}$ & $\pi$ & & $v_{10 A}\left(\mathrm{~B}_{2 g}: 853\right)+v_{17 A}\left(\mathrm{~A}_{u}: 977\right)=1830\left(\mathrm{~B}_{2 u}\right)$ \\
\hline 1957 & $\mathrm{w}$ & $\sigma$ & $\mathrm{C}$ & $v_{17 A}\left(\mathrm{~A}_{u}: 977\right)+v_{5}\left(\mathrm{~B}_{s g}: 978\right)=1955\left(\mathrm{~B}_{3 u}\right)$ \\
\hline 2070 & vw & $\pi$ & A ? & \\
\hline 2130 & $\mathrm{w}$ & $\pi$ & & \\
\hline 2200 & vw & $\sigma$ & & \\
\hline $2260 \mid$ & rw & $\sigma$ & j- & \\
\hline 2290$\}$ & Ww & $\sigma$ & C? & \\
\hline
\end{tabular}


Table 19. (Continuation)

\begin{tabular}{|c|c|c|c|c|}
\hline $\begin{array}{c}\begin{array}{c}\text { Frequency, } \\
\mathrm{cm}^{-\mathbf{1}}\end{array} \\
\end{array}$ & R. I. & Pol'n & Phase & Assignment \\
\hline $\left.\begin{array}{l}2325 \\
2375\end{array}\right\}$ & $\mathrm{vw}$ & $\pi$ & C? & \\
\hline $2410^{\prime}$ & $\mathrm{w}$ & $\pi$ & & \\
\hline 2510 & $\mathrm{vw}$ & $\sigma$ & A ? & \\
\hline 2560 & w & $\sigma$ & & \\
\hline 2625 & $\mathrm{vw}$ & $\pi$ & & \\
\hline 2690 & viv & $\pi$ & & \\
\hline 2770 & $\mathrm{vw}$ & $\pi$ & & \\
\hline 2815 & $\mathrm{vw}$ & $\pi$ & & \\
\hline 2852 & vw & $\sigma$ & $\mathrm{C}$ & $v_{s}\left(\mathrm{CH}_{2}\right)$ (crystalline) \\
\hline 2890 & $\mathrm{vw}$ & $\pi$ & & $v_{s}\left(\mathrm{CH}_{2}\right)$ (amorphous) \\
\hline 2908 & $\mathrm{mw}$ & $\pi$ & c & $v_{a}\left(\mathrm{CH}_{2}\right)$ (crystalline) \\
\hline 2970 & $\mathbf{m}$ & $\pi$ & & $\nu_{a}\left(\mathrm{CH}_{2}\right)$ (amorphous) \\
\hline 3012 & $\mathrm{w}$ & $\pi$ & C? & \\
\hline 3055 & $\mathrm{w}$ & $\pi$ & & $\nu_{20 A}^{\prime}\left\{B_{2 u}\right\}$ \\
\hline 3068 & $\mathrm{w}$ & $\sigma$ & & \\
\hline 3082 & $\mathrm{w}$ & $\sigma$ & & $v_{20 B}\left(\mathrm{~B}_{3 u}\right)$ \\
\hline 3100 & w & $\sigma$ & & \\
\hline 3130 & vw & - & & \\
\hline 3440 & $\mathrm{w}$ & $\sigma$ & & $2 \times v(\mathrm{C}=\mathrm{O})=3448$ \\
\hline 3560 & $w$ & $\pi$ & & $\nu(\mathrm{OH})$ (end groups) \\
\hline
\end{tabular}

done on deuterated polymers, polymers of varying crystallinity, and on model compounds. Nevertheless many problems of assignment have not been completely clarified. Our discussion of the spectrum of PETP will therefore center in some detail on these questions of spectral assignment, since the structural insights hinge so closely on the results of these interpretations.

The infrared spectrum of an oriented, fairly crystalline, specimen of PETP is shown in Fig. 20a [LIANG and KrIMM (115)]. The band positions, relative intensities, and polarizations are given in Table 19. Also shown in the table is the phase from which the band originates, drawn from data on intensity changes in polymers of varying crystallinity examined by a number of authors [COBBS and BURTON (36); MILler and WILLIS (133); Tobin (229); Daniels and Kitson (44); Grime and Ward (67); Miyake (134)]. In Fig. 20b, c, and d are shown spectra of various deuterated polymers; band positions will be found in the original publication [LIANG and KRIMM (115)]. For two of these deuterated polymers the intensity variation of the bands with crystallinity has also been studied [Grime and Ward (67); MiYake (735)].

The structure of crystalline PETP has been the subject of a detailed $\mathrm{x}$-ray diffraction study [DAUBENY, BUNN, and Brown (45)]. The results of this study are indicated in Fig. 21. The molecules are nearly planar and have a center of symmetry. A slight departure from planarity results from 
the $\mathrm{COO}$ group being about $12^{\circ}$ out of the plane of the benzene ring. It is also thought that the $\mathrm{CH}_{2}-\mathrm{CH}_{2}$ bond is rotated about $20^{\circ}$ around the $\mathrm{O}-\mathrm{CH}_{2}$ bond from the planar configuration, but this is less certain since the positions of the $\mathrm{CH}_{2}$ carbon atoms are not known as well as those of

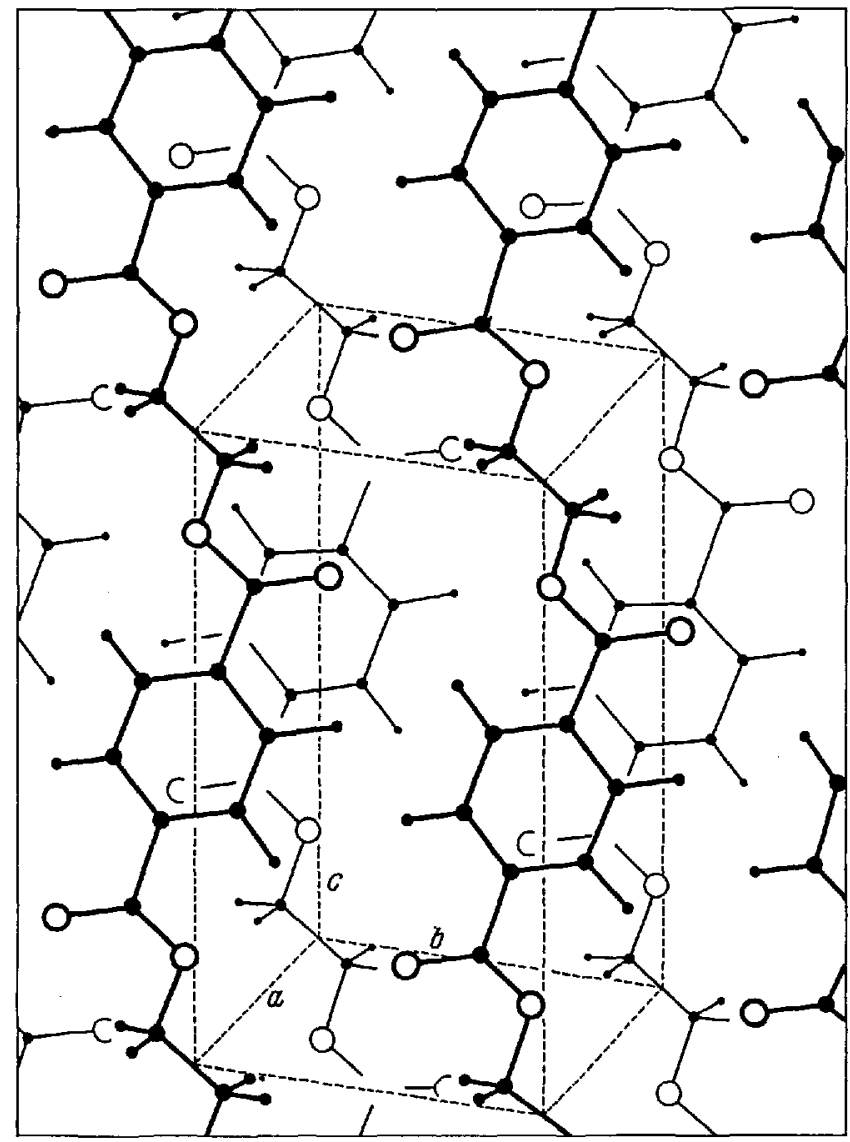

Fig. 21. Crystal structure of polyethylene terephthalate [DAUBENY, Buns, and Brows (45)]

the other carbon and oxygen atoms. Although the molecule possesses only a center of symmetry, it seems possible, to a good first approximation, to analyze some of the chain modes in terms of local symmetries [LIANG and KRIMM (115)]. Thus, for example, the vibrations of the $\mathrm{C}-\mathrm{C}_{6} \mathrm{H}_{4}-\mathrm{C}$ part of the chain appear to be classifiable in terms of the $V_{h}$ symmetry of a simple para-disubstituted benzene. The symmetry analysis shows that the infrared active modes of this unit will be split up as follows: $B_{1 u}(\sigma)-3$, $B_{2 u}(\pi)-5$, and $B_{3 u}(\sigma)-5$. Possible symmetry coordinates for these 
modes are shown in Fig. 22. The remainder of the chain is assumed to give rise to separable group modes, although it is to be recognized that in some cases such a description may not be completely adequate.

One of the characteristic features of the PETP spectrum is the relatively large number of bands whose intensity is a function of the crystallinity of the specimen. Since an interpretation of these changes can lead to information on the chain configuration in the amorphous phase, many<smiles>CC1=C(C)C(C)(C)C(C)=C1C</smiles><smiles>CC1=C(C)C2(C)C(C)C(C)(C1C)C2(C)C</smiles><smiles>CC1=C(C)[C@]2(C)C(C)=C(C)[C@]1(C)C2(C)C</smiles><smiles>CC1C(C)(C)C2(C)C(I)C1(C)C2(C)C</smiles><smiles>CC1=C(C)C(C)C(C)(C)C1C</smiles>

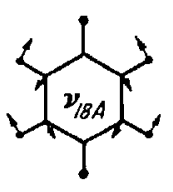

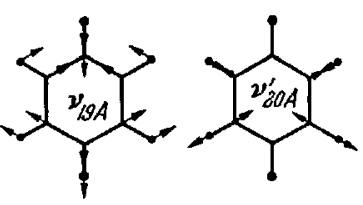
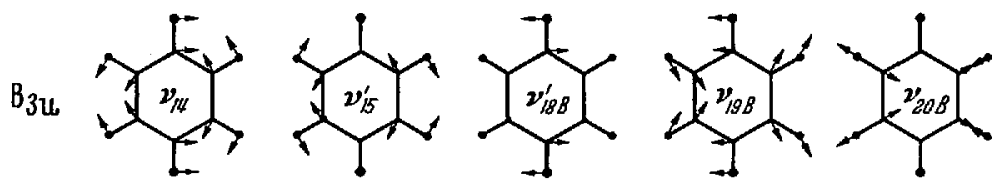

Fig. 22. Possible infrared active symmetry modes for the $\mathrm{C}-\mathrm{C}_{6} \mathrm{H}_{4}-\mathrm{C}$ portion of the polyethylene terephthalate chain [LIANG and KrIMM (175)]

workers have studied this aspect of the problem in great detail. In almost all of these cases [WARD $(237,238)$; SEIDEL (194); Grime and WARD (67); DANiElS and KITSON (44); MiYAKe (134)] it has been asserted that the bands whose intensity is a function of specimen crystallinity are associated with the $-\mathrm{OCH}_{2} \mathrm{CH}_{2} \mathrm{O}$ - portion of the chain, and that the spectral changes point to a trans configuration of this unit in the crystalline regions and a gauche configuration in the amorphous regions. However, it has been pointed out [LIANG and KRIMM (115)] that not only do several band intensities vary with crystallinity for bands definitely not associated with the $-\mathrm{OCH}_{2} \mathrm{CH}_{2} \mathrm{O}$ - unit, but the polarization of some of the bands are not consistent either with the proposed chain structure or the transgauche assignments. While these difficulties appeared serious at the time, it seems that a new synthesis of the presently available data does permit a more satisfactory analysis. The results of this analysis indicate that the chain structure is in need of some revision, and that while the band 
intensity changes may indicate trans-gauche rotational isomerism, rotations involving bonds near the benzene ring may not be negligible. Intensity changes due to intermolecular interactions in the crystal do not appear to be operable in the present case.

The assignment of the $\mathrm{CH}_{2}$ modes will be considered first, since they lead directly to the problems of structure and rotational isomerism mentioned above. The primary difficulty that has arisen is that it has appeared that all of the bands assignable to $\mathrm{CH}_{2}$ modes of the crystal phase have $\pi$ polarization. If we assume mutually perpendicular transition moments for the $\mathrm{CH}_{2}$ modes (and there seems no reason not to, in first approximation), then for a uniaxially oriented polymer, which is the case for stretched PETP [LIANG and KRIMM (112)], it has been shown [LIANG and KRIMm (113)] that it is not possible for the three perpendicular moments to all give rise to $\pi$ bands. Only the combinations $\sigma \sigma \pi$ and $\pi \pi \sigma$ are possible with polarized bands. An examination of polarization data [LIANG and KRIMM (115)] and crystallinity data [GRIME and WARD $(67)]$ in the $\nu\left(\mathrm{CH}_{2}\right)$ region, combined with the previous assignments for the other $\mathrm{CH}_{2}$ modes, now reveals that the earlier difficulties can be circumvented and a consistent interpretation proposed. The $2852, \sigma$ and $2908, \pi$ bands of the former study are revealed by the latter work to be crystallinity bands. If they are assigned to $v_{s}\left(\mathrm{CH}_{2}\right)$ and $v_{a}\left(\mathrm{CH}_{2}\right)$ respectively, then the $1473, \sigma(\mathrm{C}), 1343, \pi(\mathrm{C})$, and $845, \pi(\mathrm{C})$ bands can naturally be assigned to $\delta\left(\mathrm{CH}_{2}\right), \gamma_{w}\left(\mathrm{CH}_{2}\right)$, and $\gamma_{r}\left(\mathrm{CH}_{2}\right)$ modes, presumably of a trans $-\mathrm{OCH}_{2} \mathrm{CH}_{2} \mathrm{O}-$ unit. Not only are the $v_{s}$ and $\delta$, and $v_{a}$ and $\gamma_{r}$, polarizations alike, as they should be, but the $\pi \pi \sigma$ combination is an allowed one. It should be noted that a $\sigma \nu_{s}\left(\mathrm{CH}_{2}\right)$ mode and a $\pi \boldsymbol{v}_{a}\left(\mathrm{CH}_{2}\right)$ mode are not in agreement with the predicted dichroisms of the proposed crystal chain structure [LIANG and KRIMM) 115)], still indicating a need for a revision of the proposed structure, though perhaps not as severe as originally suggested. The remaining bands can also be interpreted satisfactorily: $v_{a}\left(\mathrm{CH}_{2}\right)-2970, \pi, \quad v_{s}\left(\mathrm{CH}_{2}\right)-2890, \pi, \quad \delta\left(\mathrm{CH}_{2}\right)-1455, \pi$, $\gamma_{w}\left(\mathrm{CH}_{2}\right)-1370, \sigma$, and $\gamma_{r}\left(\mathrm{CH}_{2}\right)-897, \pi\left(\right.$ ?). The $\nu\left(\mathrm{CH}_{2}\right)$ modes also seem to move consistently on deuteration: $2852, \sigma$ to $2250, \sigma, 2908, \pi$ to $2380, \pi$ [these two $\mathrm{CD}_{2}$ bands seem to increase in intensity with crystallinity [MrYake (135)], 2890, $\pi$ to $2118, \pi$, and $2970, \pi$ to $2190, \pi$. The frequency ratio is higher for $v_{s}$ than for $v_{a}$, as expected (see Table 4), and the $v_{s}\left(\mathrm{CD}_{2}\right)$ mode has increased in intensity relative to the $v_{a}\left(\mathrm{CD}_{2}\right)$ mode similarly in both cases. (The weak band observed at $2160 \mathrm{~cm}^{-1}$ [MrYAKE (135)] may not be a $\mathrm{CD}_{2}$ mode but an overtone: $1107+1055(\mathrm{C})=2162(\mathrm{C})$.)

While most of the above assignments are satisfactory, it is necessary to point out that some difficulties do exist [LIANG and Krimm (175)]. The assignment of the 1455 and $1473 \mathrm{~cm}^{-1}$ bands to $\delta\left(\mathrm{CH}_{2}\right)$ modes is reasonable in view of the range observed in the polymers discussed so far, viz., 
$1407-1473 \mathrm{~cm}^{-1}$. However, in polyethylene glycol [Davison (47)] in which the $-\mathrm{OCH}_{2} \mathrm{CH}_{2} \mathrm{O}$ - units are presumably in the gauche configuration, the strong band is at $1470 \mathrm{~cm}^{-1}$, the $1455 \mathrm{~cm}^{-1}$ band being very weak. Similarly, in this polymer the strong $\gamma_{w}\left(\mathrm{CH}_{2}\right)$ mode is at $1344 \mathrm{~cm}^{-1}$, and the strong $\gamma_{r}\left(\mathrm{CH}_{2}\right)$ mode is at $844 \mathrm{~cm}^{-1}$. The latter band again seems to be most reasonably associated with a gauche configuration [DAvison (47); KURODA and KuBo (107)]. On the basis of such frequency correlations it would seem that the configuration in the crystal might be the gauche rather than the trans. Perhaps this accounts for the observed polarizations of the $\boldsymbol{v}_{s}\left(\mathrm{CH}_{2}\right)$ and $v_{a}\left(\mathrm{CH}_{2}\right)$ modes. Another difficulty is the assignment of the $1343 \mathrm{~cm}^{-1}$ band to a $\gamma_{w}\left(\mathrm{CH}_{2}\right)$ mode. First, this band is much more intense in comparison to the $\delta\left(\mathrm{CH}_{2}\right)$ modes than is the case for other polymers. Second, a band appears at this position in all deuterated polymers except PE- $\mathrm{D}_{4}$-TP. If it is assumed that in the case of the $\mathrm{C}_{6} \mathrm{D}_{4}$ polymers this band is due to a deuterated ring mode, than its origin and its high intensity are difficult to explain. Likewise, the constancy of its intensity relative to the $1410 \mathrm{~cm}^{-1}$ band in both $\mathrm{C}_{6} \mathrm{D}_{4}$ polymers is difficult to account for if we assume $\gamma_{w}\left(\mathrm{CH}_{2}\right)$ to be contributing in one of these cases. This assignment therefore requires further substantiation. Although these several difficulties exist, it seems that the present set of assignments is nevertheless somewhat more satisfactory than an alternative.

The assignment of the ring modes can be made with the help of previous analyses [LIANG and KRIMM (115)], and require only some small modifications. The $B_{2 u}$ modes should show $\pi$ polarization, and of these five modes the assignment of $v_{18 A}$ and $v_{20 A}^{\prime}$ gives little difficulty. The $v_{19 A}$ mode, at $1485 \mathrm{~cm}^{-1}$ in benzene, is most probably to be assigned to the $1504 \mathrm{~cm}^{-1}$ band. In the earlier analysis the $v_{13}^{\prime}$ mode was found to be reasonably assigned to the 1343 or $1410 \mathrm{~cm}^{-1}$ bands. If we now assign the former to $\gamma_{w}\left(\mathrm{CH}_{2}\right)$, then we should choose the $1410 \mathrm{~cm}^{-1}$ band for $\nu_{13}^{\prime}$. Its constancy of position with deuteration would be consistent with this assignment. The $v_{12}$ mode cannot be identified with certainty. Some arguments [LIANG and KRIMM (115)] suggest its assignment to the $973 \mathrm{~cm}^{-1}$ band, and this would be supported by the presence of a band near this frequency in poly-p-xylylene [CORLEY, HAAS, KANE, and Livingstone (39)]. Other arguments $[\operatorname{MiyAkE}(134,135)]$ indicate that the $973 \mathrm{~cm}^{-1}$ band should be assigned to a $\nu(\mathrm{O}-\mathrm{C})$ mode of a trans $-\mathrm{OCH}_{2} \mathrm{CH}_{2} \mathrm{O}$ - unit, which seems to be in agreement with studies on polyesters [DAvison (46)] and polyethylene glycol [Davison (47)]. This assignment needs further clarification, perhaps by studies on crystalline and amorphous compounds such as $\mathrm{CH}_{3} \mathrm{OCH}_{2} \mathrm{CH}_{2} \mathrm{OCH}_{3}$. The three outof-plane $B_{1 u}$ modes of the benzene ring can be assigned fairly satisfactorily, $v_{11}$ and $v_{17 B}$ on the basis of studies on the deuterated polymers and $\nu_{16 B}$ on the basis of expected combination bands [LIANG and KRIMM 
(115)]. Of the $B_{3 u}$ modes only $v_{20 B}$ can be assigned with confidence to the $\sigma$ band at $3082 \mathrm{~cm}^{-1}$. Probable assignments can be given for two others, $v_{15}^{\prime}$ and $\boldsymbol{v}_{18 B}^{\prime}$ [LIANG and KRIMm (115)]. We will also suggest the assignment of $\nu_{19 B}$ to the weak band at $1387 \mathrm{~cm}^{-1}$. Finally, if we accept the assignment of $v_{14}$ in benzene to the $1309 \mathrm{~cm}^{-1}$ band [BRODERSEN and LANGSETH (19)], occurring at $1282 \mathrm{~cm}^{-1}$ in $\mathrm{C}_{6} \mathrm{D}_{6}$, then the $1280 \mathrm{~cm}^{-1}$ band of PETP may be associated with this mode. In the ring-deuterated polymers this band may have moved to $1300 \mathrm{~cm}^{-1}$, perhaps indicating that this vibration is mixed with others.

The above bands in the PETP spectrum are not the only ones associated with vibrations of the benzene ring; Raman-active modes appear to give rise to some of the bands, and combination bands are found [LIANG and KRIMM (115)]. Raman-active modes can become activated by loss of the center of symmetry of the p-disubstituted benzene ring in PETP, probably by rotations of the COO group away from the symmetrical position. If this is so, then it suggests another mechanism, other than rotational isomerism of the $-\mathrm{OCH}_{2} \mathrm{CH}_{2} \mathrm{O}$ - group, by means of which the amorphous chain structure differs from that in the crystalline regions. On this basis we expect such bands to be most intense in the amorphous material. Thus, for example, the assignment of the 1580 and $1617 \mathrm{~cm}^{-1}$ bands to $v_{8 A}\left(A_{1}\right)$ and $v_{8 B}\left(B_{1}\right)$ naturally suggests itself (see Fig. 17 for the approximate form of these vibrations). Other possibilities of this type are $v_{9 A}\left(A_{1}\right)$ at $1172 \mathrm{~cm}^{-1}, \nu_{4}\left(B_{2}\right)$ at $680 \mathrm{~cm}^{-1}, v_{6 B}\left(B_{1}\right)$ at $613 \mathrm{~cm}^{-1}$, and possibly $v_{6 A}\left(A_{1}\right)$ at $525 \mathrm{~cm}^{-1}$. The $1042 \mathrm{~cm}^{-1}$ band, which is also present in poly-p-xylylene, may be a $v(\mathrm{CC})$ mode which becomes active in the amorphous phase as a result of the loss of a center of symmetry. As has been shown [LIANG and KRIMM (175)] several characteristic combination bands of ring fundamentals appear in the spectrum; these are shown in Table 19. The assignment of the remaining skeletal modes can be made with confidence in only a few cases. The results of arguments concerning these assignments [LIANG and KRIMM (115)] are shown in Table 19.

Although some of the assignments of the bands in the spectrum of PETP cannot be made with great certainty at this time, it appears that the main outlines of a satisfactory spectral interpretation have been established. At least the problems involved in achieving this now stand out more clearly. As has been noted in the previous discussion, these assignments hold the key to a deeper understanding of the molecular chain structure, both in the crystalline and in the amorphous phases.

\section{Conclusion}

In Part IV of this article we have attempted to show how the methods discussed in Part III can be, and have been, applied to the detailed analysis of the spectra of some high polymers. The coverage was not 
intended to be exhaustive, but rather to at least bridge the gap between the simpler and the more complex polymers. For references to discussions on other polymers a recent review is available [SchNeLL (193)].

What conclusions can be drawn from the results of the spectral analyses discussed here? It seems fair to say that the kind of approach to polymer spectra which we have been considering reveals a new dimension of power in the spectroscopic tool of analysis. As we have seen, the achievement of a complete set of assignments for a polymer can lead to deeper insights into questions of structure and internal forces in the chain, both in the crystalline and the amorphous regions. Such information is also of primary importance if the spectrum is to be used to full advantage in following changes in structure and properties contingent on various kinds of physical and chemical treatments. In general, the extent to which such detailed analyses have been achieved is less than is desirable. This is primarily because the methods of analysis discussed in Part III have not always been prosecuted to their fullest. Spectral studies of deuterated polymers are only now being undertaken more frequently, and even then only of the fully deuterated polymer when studies of partially deuterated polymers are often more important. Polarization studies are not as common yet as they should be. And as has been pointed out, there is a significant absence of Raman data on polymers. The theme underlying the present developments in the spectroscopy of high polymers is that fuller application of presently available techniques can lead to detailed understanding of the spectrum, which in turn opens up potentially more powerful uses of the infrared spectrum in gaining an understanding of the physical and chemical properties of polymers.

This investigation was supported in part by a PHS research grant, A-2830, from the National Institute of Arthritis and Metabolic Diseases, Public Health Service.

\section{References}

1. ABE, K., and K. YANAGISAWA: Infrared spectra of melted polypropylene film. J. Polymer Sci. 36, 536-539 (1959).

2. Acquista, N., and E. K. Plyler: Infrared measurements with a cesium iodide prism. J. opt. Soc. Amer. 43, 977-979 (1953).

3. Ahonen, C. O.: A theoretical evaluation of normal frequencies of vibration of the isomeric octanes. J. chem. Phys. 14, 625-636 (1946).

4. Ambrose, E. J., and A. Elliotr: The structure of synthetic polypeptides. II. Investigation with polarized infrared spectroscopy. Proc. roy. Soc. A 205, $47-60$ (1951).

5. - - Infrared spectra and structure of fibrous proteins. Proc. roy. Soc. A 206, 206-219 (1951).

6. - - and R. B. Temple: The use of polarized infrared radiation in the study of doubly oriented long-chain polymers. Proc. roy. Soc. A 199, 183-198 (1949).

7. A. P. I. Spectra (American Petroleum Institute Research Project 44) : Catalog of Raman spectral data. Petroleum Research Laboratory, Carnegie Institute of Technology, 1956.

Fortschr. Hochpolym.-Forsch., Bd. 2 
8. Bamford, C. H., A. Elliott, and W. E. Hanby: Synthetic polypeptides. New York: Academic Press Inc., 1956.

9. Barriol, J., et J. Chapelle: Contribution a l'etude des vibrations des longues chaines carbonées. J. phys. radium 8, 8-13 (1947).

10. Barrow, G. M.: Remarks on the interpretation of the $\mathrm{CH}_{2}$ vibrational frequencies of n-paraffins. J. chem. Phys. 19, 345-346 (1951).

11. Bartholomé, E., u. E. Teller: Modellmäßige Berechnung von Eígenschwingungen organischer Kettenmoleküle. Z. physik. Chem. B 19, 366-388 (1932).

12. BeER, M. : Quantitative interpretation of infrared dichroism in partly oriented polymers. Proc. roy. Soc. A 236, 136-140 (1956).

13. Bellamy, L. J.: The infrared spectra of complex molecules. 2nd edition. New York: John Wiley and Sons, 1958.

14. Bernstein, H. J.: The calculation of the change in vibrational frequency due to changes in mass, geometry, and potential constants of a molecule. Canad. J. Chem. 29, 284-295 (1951).

15. Bhagavantam, S., and T. Venkatarayudu: Theory of groups and its application to physical problems. Waltair: Andhra University, 1951.

16. Blout, E. R. : Microspectroscopy, in Technique of organic chemistry. Vol. I, Part III, Chap. XXXIII, pp. 2179-2224. 2nd edition. New York: Interscience Publishers, 1954.

17. - and R. Karplus: The infrared spectrum of polyvinyl alcohol. J. Amer. chem. Soc. 70, 862-864 (1948).

18. Born, M., and K. Huang: Dynamical theory of crystal lattices. Oxford University Press, 1954.

19. Brodersen, S., and A. LANGSErH: The infrared spectra of benzene, symbenzene- $d_{3}$, and benzene- $d_{6}$. Mat. Fys. Skr. Dan. Vid. Selsk. 1, No. 1, 1-45 (1956).

20. Brown, J. K., and N. SHePpard: Infrared spectroscopic studies of rotational isomerism. I. The assignment of the fundamental frequencies of some ethylene dihalides. Trans. Faraday Soc. 48, 128-137 (1952).

21. - - A calculation of the characteristic $\mathrm{C}-\mathrm{H}$ out-of-plane deformation freqiencies of substituted ethylenes. Trans. Faraday Soc. 51, 1611-1613 (1955).

22. - - and D. M. Simpson: The interpretation of the vibrational spectra of the n-parafins. Disc. Faraday Soc. 9, 261-274 (1950).

23. - - - The interpretation of the infrared and Raman spectra of the n-paraffins. Phil. Trans. roy. Soc. A 247, 35-58 (1954).

24. BRÜGEL, W.: Einführung in die Ultrarotspektroskopie. Darmstadt: D. Steinkopff, 1954.

25. BRYANT, W. M. D., and R. C. VOTER: The molecular structure of polyethylene. II. Determination of short chain branching. J. Amer, chem. Soc. 75, 6113-6118 (1953).

26. Bunn, C. W.: Structure of long-chain normal paraffin hydrocarbons. "Shape" of $\mathrm{CH}_{2}$ group. Trans. Faraday Soc. 35, 482-491 (1939).

27. - Crystal structure of polyvinyl alcohol. Nature (Lond.) 161, 929-930 (1948).

28. - and D: R. Holmes: Chain configurations in crystals of simple linear polymers. Disc. Faraday Soc. 25, 95-103 (1958).

29. - and E. R. Howells: Structures of molecules and crystals of fluorocarbons. Nature (Lond.) 174, 549-551 (1954).

30. - - Chain configuration in crystalline vinyl polymers. J. Polymer Sci. 18, 307-310 (1955).

31. Cannon, C. G.: The nature of hydrogen bonding. Spectrochim. Acta 10, $341-368$ (1958).

32. Chapman, D.: The $720 \mathrm{~cm}^{-1}$ band in the infrared spectra of crystalline longchain compounds. J. chem. Soc. 1957, 4489-4491. 
33. Crarney, E.: Dichroic ratio measurements in the infrared region. J. opt. Soc. Amer. 45, 980-983 (1955).

34. ClaAsen, H. H.: Vibration spectra and normal coordinate treatment of perfluorocyclobutane. J. chem. Phys. 18, 543-551 (1950).

35. Clark, C.: Infrared spectrophotometry, in Physical techniques in biological research, ed. G. Oster and A. W. Pollister. Vol. I, pp. 205-323. New York: Academic Press 1955.

36. CosBs, W. H., JR., and R. L. BuRton: Crystallization of polyethylene terephthalate. J. Polymer Sci. 10, 275-290 (1953).

37. Corthup, N. B.: Spectra-structure correlations in the infrared region. J. opt. Soc. Amer. 40, 397-400 (1950).

38. Conn, G. K. T., and G. K. EATos: On polarization by transmission with particular reference to selenium films in the infrared. J. opt. Soc. Amer. 44, $553-557$ (1954).

39. Corley, R. S., H. C. HaAs, M. W. Kane, and D. I. Livingstone: Preparation and properties of poly-p-xylylene. J. Polymer Sci. 13, 137-156 (1954).

40. Coulson, C. A.: Valence. London: Oxford, 1952.

41. - Some theoretical considerations about vibrational band intensities. Spectrochim. Acta 14, 161-180 (1959).

42. CRAWFORD, B. L., and S. R. BRINkLEY: Force constants in some organic molecules. J. chem. Phys. 9, 69-75 (1941).

42a. Cross, L. H., R. B. Richards, and H. A. WiLlis: The infrared spectrum of ethylene polymers. Disc. Faraday Soc. 9, 235-245 (1950).

43. Cross, P. C., and J.H. VAN VLECK: Molecular vibrations of three particle systems with special applications to the ethyl halides and ethyl alcohol. J. chem. Phys. 1, 350-356 (1933).

44. Daniels, W. W., and R. E. Kirson: Infrared spectroscopy of polyethylene terephthalate. J. Polymer Sci. 33, 161-170 (1958).

45. Daubeny, R. DE P., C. W. Bunn, and C. J. Brown: The crystal structure of polyethylene terephthalate. Proc. roy. Soc. A226, 531-542 (1954).

46. Davison, W. H. T.: Infrared spectra and crystallinity. Part. I. Polyesters. J. chem. Soc. 1955, 2428-2431.

47. - Infrared spectra and crystallinity. Part III. Polyethylene glycol. J. chem. - Soc. 1955, 3270-3274.

48. Decius, J. C.: Force constants for some halomethanes. J. chem. Phys. 16, $214-223(1948)$.

49. Dennison, D. M.: The infrared spectra of polyatomic molecules. Part II. Rev. mod. Phys. 12, 175-214 (1940).

50. EDGELl, W. F.: The isotope effect and the ratio rule. J. chem. Phys. 13, 539-546 (1945).

51. ElilotT, A.: Infrared dichroism of synthetic polypeptides. Nature (Lond.) $172,359-360\langle 1953\rangle$.

51a. - The infra-red spectra of polymers. Advances in Spectroscopy 1, 214-287 (1959).

52. - E. J. Ambrose, and R. B. Temple: The polarization of infrared radiation. J. opt. Soc. Amer. 38, 212-216 (1948).

53. - - - Polarized infrared radiation as an aid to the structural analysis of long-chain polymers. I. J. chem. Phys. 16, 877-886 (1948).

54. FARMER, V. C.: Effects of grinding during the preparation of alkali-halide disks on the infrared spectra of hydroxylic compounds. Spectrochim. Acta 8, $374-389$ (1957).

55. Folt, V. L., J. J. Shipman, and A. R. Berens : Private communication, 1959. 
56. Fox, J. J., and A. E. MARTIN: Investigations of infrared spectra. Determination of $\mathrm{C}-\mathrm{H}$ frequencies $\left(\sim 3000 \mathrm{~cm}^{-1}\right)$ in paraffins and olefins, with some observations on "polythenes". Proc. roy. Soc. A 175, 208-233 (1940).

57. Francis, S. A. : Absolute intensities of characteristic infrared absorption bands of alipliatic hydrocarbons. J. chem. Phys. 18, 861-865 (1950).

58. Fraser, R. D. B.: The interpretation of infrared dichroism in fibrous protein structures. J. chem. Phys. 21, 1511-1515 (1953).

59. - Interpretation of infrared dichroism in fibrous proteins - the $2 \mu$ region. J. chem. Phys. 24, 89-95 (1956).

60. - Interpretation of infrared dichroism in axially oriented polymers. J. chem. Phys. 28, 1113-1115 (1958).

61. - Determination of transition moment orientation in partially oriented polymers. J. chem. Phys. 29, 1428 - 1429 (1958).

62. - and W. C. PRICE: Infrared dichroism and protein structure. Nature (Lond.) $170,490-491$ (1952).

63. Fuchs, W., und D. Lours: Die Struktur chlorierter Polyvinylchloride. Makromol. Chem. 22, 1-30 (1957).

64. FULLER, C. S. : The investigation of synthetic linear polymers by $\mathbf{x}$-rays. Chem. Rev. 26, 143-167 (1940).

65. Glatr, L., and J. W. Ellis: Near infrared pleochroism. II. The $0.8-2.5 \mu$ region of some linear polymers. J. chem. Phys. 19, 449—457 (1951).

66. - D. S. Webber, C. Seaman, and J. W. Eltis: Near infrared pleochroism. I. The perturbed $\mathrm{O}-\mathrm{H}$ and $\mathrm{C}-\mathrm{H}$ modes in polyvinyl alcohol. J. chem. Phys. 18, $413-416(1950)$.

67. GRIME, D., and I. M. WARD: The assignment of infrared absorptions and rotational isomerism in polyethylene terephthalate and related compounds. Trans. Faraday Soc. 54, 959-971 (1958).

68. Grisenthwaite, R. J., and R. F. Hunter: Infrared spectra of polyvinyl chloride samples prepared at temperatures between $-80^{\circ}$ and $+45^{\circ} \mathrm{C}$. Chem. and Ind. $719-720$ (1958).

69. Gross, E. F.: The vibration spectrum of the hydrogen bond, in Hydrogen bonding, ed. D. HadžI, pp. 203-209. New York: Pergamon Press, 1959.

70. HAAS, H. C.: A note on the infrared absorption spectrum of polyvinyl alcohol. J. Polymer Sci. 26, 391-393 (1957).

71. HALFORD, J. O.: Stretching force constant of the carbonyl bond in unconjugated ketones. J. chem. Phys. 24, 830-833 (1956).

72. HALFord, R. S.: Motions of molecules in condensed systems: I. Selection rules, relative intensities, and orientation effect for Raman and infrared spectra. J. chem. Phys. 14, 8-15 (1946).

73. - The influence of molecular environment on infrared spectra. Ann. N. Y. Acad. Sci. 69, 63-69 (1957).

74. Halverson, F.: The use of deuterium in the analysis of vibrational spectra. Rev. mod. Phys. 19, 87-131 (1947).

75. Hermans, P. H., and A. Weidinger: Estimation of crystallinity of some polymers from $x$-ray intensity measurements. J. Polymer Sci. 4, 709-723 (1949).

76. Herzierg, G.: Infrared and Raman spectra of polyatomic molecules. New York: D. Van Nostrand Co., 1945.

77. HigGs, P. W.: The vibration spectra of helical molecules: infrared and Raman selection rules, intensities and approximate frequencies. Proc. roy. Soc. A220, $472-485$ (1953).

78. Hodgkins, J. E.: Infrared analysis of a cyclopropane polymer. J. org. Chem. 23, 1369-1370 (1958). 
79. Hornig, D. F.: The vibrational spectra of molecules and complex ions in crystals. I. General theory. J. chem. Phys. 16, 1036-1076 (1948).

80. - Infrared spectra of crystals at low temperatures. Disc. Faraday Soc. 9, $115-124(1950)$.

81. Hummel, D.: Kunststoff-, Lack- und Gummi-Analyse. München: Carl Hanser Verlag, 1958.

82. JoNES, R. N., and C. SANDORFY: The application of infrared and Raman spectrometry to the elucidation of molecular structure, in Technique of organic chemistry, Vol. IX, ed. W. West. New York: Interscience Publishers, 1956.

83. Kagarise, R. E.: Relation between the electronegativities of adjacent substituents and the stretching frequency of the carbonyl group. J. Amer. chem. Soc. 77, 1377-1379 (1955).

84. KASSEL, L. S.: The modes of vibration of butane and pentane. "Free rotation" about carbon-carbon bonds and a new type of stereoisomerism. J. chem. Phys. 3, 326-335 (1935).

85. KAYE, W.: Near-infrared spectroscopy. II. Instrumentation and technique. Spectrochim. Acta 7, 181-204 (1955).

86. Keith, H. D., F. J. Padden JR., N. M. Walter, and H. W. Wyckoff: Evidence for a second crystal form of polypropylene. J. appl. Phys. 30, $1485-1488$ (1959).

87. KeLLNER, L.: The vibrations of an infinitely long chain of $\mathrm{CH}_{2}$-groups and the infrared spectrum of polythene. Proc. Phys. Soc. A 54, 521-535 (1951).

88. Kendall, D. N., R. R. Hampton, H. HausdorfF, and F. Pristera: Catalog of infrared spectra of plasticizers. Appl. spectroscopy 7, 179-196 (1953).

89. King, G. W., R. M. Hainer, and H. O. McMahon: Infrared absorption spectra of some polymers at liquid-helium temperatures. J. appl. Phys. 20, $559-563$ (1949).

90. KING, W. T., I. M. MILLs, and B. CRAwFord: Normal coordinates in the methyl halides. J. chem. Phys. 27, 455-457 (1957).

91. KIRKwoon, J. G.: The skeletal modes of vibration of long chain molecules. J. chem. Phys. 7, 506-509 (1939).

92. Kohlrausch, K. W..F.: Ramanspektren. Leipzig: Akademische Verlagsgesellschaft Becker \& Erler, 1943.

93. KRImm, $\mathrm{S}$.: Infrared dichroism in the $13.8 \mu$ band of ${ }_{n}-\mathrm{C}_{36} \mathrm{H}_{74}$ single crystals and polyethylene. J. chem. Phys. 22, 567-568 (1954).

94. - The infrared spectrum of polyethylene. Phys. Rev, 94, 1426 (1954).

95. - Frequency shift of the CO stretching band in polypeptides and proteins. J. chem. Phys. 23, 1371-1372 (1955).

96. - Infrared spectra and the structure of high polymers. SPE J. 15, 797-804 (1959).

97. - An approximate isotopic frequency rule and its application to the spectra of complex molecules. J. chem. Phys., 32, 1780-1783 (1960).

98. - Note on infrared dichroism of axially oriented polymers. J. chem. Phys., $32,313(1960)$.

99. - and A. V. ToBolsky: Quantitative $\mathrm{x}$-ray studies of order in amorphous and crystalline polymers. Quantitative $x$-ray determination of crystallinity in polyethylene. J. Polymer. Sci. 7, 57-76 (1951).

100. - and G. B. B. M. SutherLAND: Far infrared spectra of high polymers. Symposium on molecular structure and spectroscopy. Columbus, Ohio, 1952.

101. - and C. Y. LIANG: Infrared spectra of high polymers. IV. Polyvinyl chloride, polyvinylidene chloride, and copolymers. J. Polymer Sci. 22, 95-112 (1956).

102. - - and G. B. B. M. SutherLaND: Infrared spectra of high polymers. II. Polyethylene. J. chem. Phys. 25, 549-562 (1956). 
103. Krimm, S., C. Y. Liang, and G. B. B. M. SutherLand: Assignment of the OH in-plane deformation frequency. J. chem. Phys. 25, 778 (1956).

104. - - - Infrared spectra of high polymers. V. Polyvinyl alcohol. J. Polymer Sci. 22, 227-247 (1956).

105. - A. R. Berens, V. L. Folt, and J. J. Shipman: Assignment of the C-Cl stretching modes in the infrared spectrum of polyvinyl chloride. Chem. and Ind. $1512-1513$ (1958).

106. - - C Carbon-chlorine stretching modes of polyvinyl chloride. Chem. and Ind. 433 (1959).

107. KURoDA, Y., and $\mathrm{M}$. KUBO: $\mathrm{CH}_{2}$ rocking vibrations and the structure of polyethylene glycols. J. Polymer Sci. 36, 453-459 (1959).

108. LagemanN, R. T., and T. G. Miller: Thallium bromide-iodide (KRS-5) as an infrared polarizer. J. opt. Soc. Amer. 41, 1063-1064 (1951).

109. Lecomte, J.: Spectroscopie dans l'infrarouge, in Handbuch der Physik, ed. S. FLÜGGE. Vol. XXVI, pp. 244-937. Berlin: Springer-Verlag 1958.

110. LIANG, C. Y.: Selection rules for the vibrational spectra of high polymers. J. mol. Spectroscopy 1,61-68 (1957).

111. -- and S. Krimm: Infrared spectra of high polymers. III. Polytetrafluoroethylene and polychlorotrifluoroethylene. J. chem. Phys. 25, 563-571 (1956).

112. - - Orientation in polyethylene terephthalate film. J. chem. Phys. 27, $327-328$ (1957).

113. - - Infrared dichroism of mutually perpendicular normal modes in oriented high polymers. J. chem. Phys. 27, 1437-1438 (1957).

114. - - Infrared spectra of high polymers. VI. Polystyrene. J. Polymer Sci. $27,241-254(1958)$.

115. - - Infrared spectra of high polymers. IX. Polyethylene terephthalate. J. mol. Spectroscopy 3, 554-574 (i959).

116. - and F. G. Pearson: Approximate normal vibrations of crystalline polyvinyl alcohol. J. Polymer Sci. 35, 303-307 (1959).

117. - S. KRIMM, and G. B. B. M. SutherLAND: Infrared spectra of high polymers. I. Experimental methods and general theory. J. chem. Phys. 25, 543-548(1956).

118. - G. B. B. M. SurherLand, and S. KRImM: Selection rules and frequencies of the skeletal vibrations of long chain polymers in the crystalline state. J. chem. Phys. 22, 1468-1469 (1954).

119. Lippincott, E. R., and R. Schroeder: One-dimensional model of the hydrogen bond. J. chem. Phys. 23, 1099-1106 (1955).

120. LoRD, R. C., and F. A. Millek: Factors influencing vibrational frequencies of molecules: intramolecular effects. Appl. Spectroscopy 10, 115-123 (1956).

121. - and T. K. McCubBin JR.: Infrared spectroscopy from 5 to 200 microns with a small grating spectrometer. J. opt. Soc. Amer. 47, 689-697 (1957).

122. MacGillavry, C. H.: Anisotropy in the so-called amorphous part of polyvinyl alcohol. Rec. trav. chim. 69, 509-514 (1950).

123. Makas, A. S., and W. A. ShURCLIFF: New arrangement of silver chloride polarizer for the infrared. J. opt. Soc. Amer. 45, $998-999$ (1955).

124. MARshall, H. W., and K. J. MikLUS: A far infrared grating spectrometer. Symposium on molecular structure and spectroscopy. Columbus, Ohio, 1958.

125. Martin, J. M., JR., R. W. B. Johnston, and M. J. O'NEAL: Infrared absorption of solid state n-paraffins - relationship to crystalline type. Spectrochim. Acta 12, 12-16 (1958).

126. MARVEL, C. S., J. H. SAMpLE, and M. F. Roy: The structure of vinyl polymers. VI. Polyvinyl halides. J. Amer. chem. Soc. 61, 3241-3244 (1939).

127. Matthews, J. L., H. S. Peiser, and R. B. Richards: The $x$-ray measurement of the amorphous content of polythene samples. Acta Cryst. 2, 85-90 (1949). 
128. McCubBin, T. K., JR., and W. M. Sinton: A twelve-inch far infrarcd grating spectrometer. J. opt. Soc. Amer. 42, 113-115 (1952).

129. Meister, A. G., F. F. Cleveland, and M. J. Murray: Interpretation of the spectra of polyatomic molecules by use of group theory. Amer. J. Phys. 11, 239-247 (1943).

130. - J. M. Dowling, and A. J. BIELecki: Substituted methanes. XXVII. J. chem. Phys. 25, $941-942$ (1956).

131. - S. E. Rosser, and F. F. CIEVELAND: Substituted methanes. I. Raman and infrared spectral data, assignments, and force constants for some tribromomethanes. J. chem. Phys. 18, 346-356 (1950).

132. MERTZ, L.: Grating polarizers in the wavelength range $100-500$ microns. J. opt. Soc. Amer. 43, 220 (1953).

133. MilLer, R. G. J., and H. A. WILLIS: An independent measurement of the amorphous content of polymers. J. Polymer Sci. 19, 485-494 (1956).

134. MIYAKE, A.: The infrared spectrum of polyethylene terephthalate. I. The effect of crystallization. J. Polymer Sci. 38, 479-495 (1959).

135. - The infrared spectrum of polyethylene terephthalate. II. Polyethylene- $d_{4}$ terephthalate. J. Polymer Sci. 38, 497-512 (1959).

136. Miyazawa, T., T. Shimanouchi, and S. Mizushima: Normal vibrations of $\mathrm{N}$-methylacetamide. J. chem. Phys. 29, 611-616 (1958).

137. Mizusirima, S.: Structure of molecules and internal rotation. New York: Academic Press Inc., 1954.

138. - and T. Simanouti: Raman frequencies of n-parafin molecules. J. Amer. chem. Soc. 71, 1320-1324 (1949).

139. - T. Shimanouchi, K. Nakamura, M. Hayashi, and S. Tsuchiya: C-Cl stretching frequencies in relation to rotational isomerism. J. chem. Phys. 26, 970-971 (1957).

140. Mooney, R. C. L.: An x-ray study of the structure of polyvinyl alcohol. J. Amer, chem. Soc. 63, 2828-2832 (1941).

140a. Morero, D., E. Mantica, F. Cinmpelli, and D. Sianesr: Spettri infrarossi di polimeri stereoordinati di monomeri vinilaromatici. 1. Polistirilo isotattico. Nuovo Cimento Supplemento 15, Ser. 10, 122-135 (1960).

141. Moxnihan, R. E.: The molecular structure of perfluorocarbon polymers. Infrared studies on polytetrafluoroethylene. J. Amer. chem. Soc. 81, 1045 1050 (1959).

141a. NaGaI, E., S. Kuribayashi, M. Shiraki, and M. Ukita: Infrared spectra of the stereoisomers of pentane-2,4-diol as a model for polyvinyl alcohol. J. Polymer Sci. 35, 295-299 (1959).

142. Naito, K., I. Nakagawa, K. Kuratani, I. Ichishima, and S. Mizushima: Infrared and Raman spectra of 1,1,2,2,-tetrachlorethane; calculation of normal vibration. J. chem. Phys. 23, 1907-1910 (1955).

143. NARITA, S., and K. OKUDA: Unit cell dimensions of polyvinylidene chloride and polyvinylidene bromide. J. Polymer Sci. 38, 270-272 (1959).

144. - S. Ichinohe, and S. Exомото: Infrared spectrum of polyvinyl chloride. I. J. Polymer Sci. 37, 273-280 (1959).

145. - - Infrared spectrum of polyvinyl chloride. II. J. Polymer Sci. 37, $281-294$ (1959).

146. - - - Infrared spectra of vinylidene chloride - vinyl chloride copolymer. J. Polymer Sci. 36, 389_-405 (1959).

147. - - - Infrared spectrum of polyvinylidene chloride, I. J. Polymer Sci. 37, $251-261$ (1959).

148. - - - Infrared spectrum of polyvinylidene chloride II. J. Polymer Sci. 37, 263-271 (1959). 
149. Natta, G.: Une nouvelle classe de polymères d' $\alpha$-oléfines ayant une régularité de structure exceptionnelle. J. Polymer Sci. 16, 143-154 (1955).

150. - Progress in five years of research on stereospecific catalysis. SPE J. 15, $373-382$ (1959).

151. - and P. Corradini: Sulla struttura cristallina di un nuovo tipo di polipropilene. Atti Accad. naz. Lincei, Mem. Cl. Sci. fisiche mat. natur. Sez. Ir, (8) $4,73-80(1955)$.

152. - - Sulla struttura cristallina del polistirolo isotattico. Atti Accad. naz. Lincei Rend., Cl. Sci. fisiche mat. natur. 18, 19-27 (1955).

153. - - Kristallstruktur des isotaktischen Polystyrols. Makromolek. Chem. 16, $77-80$ (1955).

154. - - The structure of crystalline 1,2-polybutadiene and of other "syndyotactic polymers". J. Polymer Sci. 20, 251-266 (1956).

154a. - - Structure and properties of isotactic polypropylene. Nuovo Cimento Supplemento 15, Ser. 10, 40-51 (1960).

154b. - - and I. W. Bassi: Crystal structure of isotactic polystyrene. Nuovo Cimento Supplemento 15, Ser. 10, 68-82 (1960).

155. - - and M. CESARI: Sulla struttura cristallina del polipropilene isotattico. Atti Accad. naz. Lincei Rend., Cl. Sci. fisiche mat. natur. 21, 365-372 (1956).

156. Newman, R., and R. S. Halford: An efficient, convenient polarizer for infrared radiation. Rev. Sci. Inst. 19, 270-271 (1948).

157. - - Motions of molecules in condensed systems. VII. The infrared spectra for single crystals of ammonium nitrate and thallous nitrate in polarized radiation. J. chem. Phys. 18, 1276-1290 (1950).

158. Nichols, J. B.: X-ray and infrared studies on the extent of crystallization of polymers. J. appl. Phys. 25, 840-847 (1954).

159. Nielsen, J. R.: Private communication, 1959.

160. - and A. H. WoolletT: Vibrational spectra of polyethylenes and related substances. J. chem. Phys. 26, 1391-1400 (1957).

161. - and R. F. Holland: The infrared active methylene wagging fundamental in crystalline polyethylene. Symposium on molecular structure and spectroscopy. Columbus, Ohio, 1959.

162. Oetjen, R. A., W. H. Haynie, W. M. Ward, R. L. Hansler, H. E. SchauWECKER, and E. E. BELL: An infrared spectrograph for the use in the 40 to 150 micron spectral region. J. opt. Soc. Amer. 42, 559-566 (1952).

163. Palm, A.: Raman spectrum of polystyrene. J. phys. Chem. 55, 1320—1324 (1951).

164. Pauling, L.: Factors affecting the structure of hemoglobins and other proteins. Symposium on protein structure, ed. A. Neuberger. p. 17. New York: John Wiley 1958.

165. - and E. B. Wilson JR.: Introduction to quantum mechanics. Chap. X. New York: McGraw-Hill Book Co., 1935.

166. Parodi, M.: Calcul des frequences propres des chaînes aliphatiques normales. Application à la structure des paraffines. J. phys. radium 2, 58-62 (1941).

167. Peraldo, M.: Interpretazione dello spettro infrarosso del polipropilene isotattico. Gazz. chim. ital. 89, $798-808$ (1959).

168. Person, W. B., and G. C. Pimentel: Thermodynamic properties and the characteristic $\mathrm{CH}_{2}$ frequencies of n-paraffins. J. Amer. chem. Soc. 75, 532-538 (1953).

169. Peters, C. W., T. F. Zipf, and P. V. Deibel: Microwave measurements of the intensity distribution of echelette diffraction gratings. J. opt. Soc. Amer. 43, 816 (1953).

170. PFund, A. H. : Improved infrared polarizer. J. opt. Soc. Amer. 37, 558-559 (1947). 
171. Pierce, R. H. H., JR., E. S. Clark, J. F. Whitney, and W. M. D. Bryant: Crystal structure of polytetrafluoroethylene. Abstracts of Amer. chem. Soc., 130 th meeting, September 1956.

172. Pimentel, G. C., and W. A. Klemperer: Infrared spectrum of solid n-octane$1,1,1,8,8,8-d_{\mathfrak{c}}$. J. chem. Phys. 23, 376-378 (1955).

172a. - and A. L. McClellan: The hydrogen bond. San Francisco: W. H. Freeman and Co., 1960.

173. - A. L. McClellan, W. B. Person, and O. Schnepp: Interpretation of the infrared spectrum of a molecular crystal: naphthalene. J. chem. Phys. 23, 234-237 (1955).

174. Pitzer, K. S.: The vibrational frequencies and thermodynamic functions of long chain hydrocarbons. J. chem. Phys. 8, 711-720 (1940).

175. Plyler, E. K., and N. Acguista: Infrared measurements from 50 to $125 \mathrm{mi}-$ crons. J. Res, nat. Bur. Standards 56, 149-153 (1956).

176. - - and L. R. BLAINE: Infrared measurements with a small grating from 100 to 300 microns. J. Res. nat. Bur. Standards 60, 55-57 (1958).

177. Pokrovsky, E. I., and I. P. Kotova: Investigation of crystallinity of some polymers by infrared spectroscopy. Zh. tekh. Fiz. 26, 1456-1460 (1956) [see Soviet Physics, Tech. Physics 1, 1417-1421 (1957)].

178. - and M. V. VOLKENSHTEIN: Investigation of isotactic polypropylene by means of infrared spectra. Dokl. Akad. Nauk SSSR 115, 552-553 (1957) [see Proc. Acad. Sci. USSR, Sect. Phys. Chem. 115, 517-518 (1957)].

179. Primas, H., und Hs. H. GUNTHARD: Die Infrarotspektren von Kettenmolekeln der Formel $\mathrm{R}^{\prime} \mathrm{CO}\left(\mathrm{CH}_{2} \mathrm{CH}_{2}\right)_{n} \mathrm{COR}^{\prime \prime}$. I. Rocking- und Twisting-Grundtöne. Helv, chim. Acta 36, 1659-1670 (1953).

180. - - Die Infrarotspektren von Kettenmolekeln der Formel $\mathrm{R}^{\prime} \mathrm{CO}\left(\mathrm{CH}_{2} \mathrm{CH}_{2}\right)_{n}$ $\mathrm{COR}^{\prime \prime}$. II. Die Normalschwingungen des Symmetrietypus $B_{u}$. Helv, chim. Acta 36, 1791-1803 (1953).

181. Randali, H. M.: The spectroscopy of the far infrared. Rev. mod. Phys. 10, $72-85$ (1938).

182. - R. G. Fowler, N. Fuson, and J. R. DANGL: Infrared determination of organic structures. New York: D. Van Nostrand Co., 1949.

183. RANDLE, R. R., and D. H. WhIFFEN: The characteristic vibration frequencies of substituted benzenes, in Molecular spectroscopy. London: The Institute of Petroleum, 1955, pp. 111-128.

184. Rasmussen, R. S.: Vibrational frequency assignments for paraffin hydrocarbons; infrared absorption spectra of the butanes and pentanes. $\mathrm{J}$. chem. Phys. 16, 712-727 (1948).

185. Reinhardt, R. C. : Vinylidene chloride polymers. Ind. Eng. Chem. 35, 422-428 (1943).

186. Robinson, T. S.: Optical constants by reflection. Proc. Phys. Soc. B65, 910-911 (1952).

187. - and W. C. PRICE: The determination of infrared absorption spectra from reflection measurements. Proc. Phys. Soc. B 66, 969 - 974 (1953).

188. - - The interpretation and use of infrared reflection spectra, in Molecular spectroscopy. London: The Institute of Petroleum, 1955, pp. 211-223.

189. Rosenthal, J. E., and G. M. Murphy: Group theory and the vibrations of polyatomic molecules. Rev, mod. Phys. 8, 317-346 (1936).

190. RUGG, F. M., J. J. SMIth, and L. H. WARTMAN: Infrared spectrophotometric studies on polyethylene. I. Structure. J. Polymer Sci. 11, 1-20 (1953).

191. - - and R. C. BACON : Infrared spectrophotometric studies on polyethylene. II. Oxidation. J. Polymer Sci. 13, 535-547 (1954).

192. SANds, J. D., and G. S. Turner: New development in solid phase infrared spectroscopy. Anal. Chem. 24, 791-793 (1952). 
193. SchnelL, GG.: Neuere physikalische Untersuchungen an Hochpolymeren. III. Ultrarotspektroskopische Untersuchungsergebnisse. Ergebn. exakt. Naturwiss. 31, 270-330 (1959).

194. SEIDEL, B.: Spektroskopische Beobachtungen an linearen und zyklischen Oligoestern aus Terephthalsäure und Glykol. Z. Elektrochem. 62, 214-219 (1958).

194a. Sheppard, N.: Rotational isomerism about $\mathrm{C}-\mathrm{C}$ bonds in saturated molecules as studied by vibrational spectroscopy. Advances in Spectroscopy 1, $288-353$ (1959).

195. Sheppard, N., and D. M. Simpson: The infrared and Raman spectra of hydrocarbons, Part II. Paraffins. Quart. Rev. 7, 19-55 (1953).

195a. Shimanouchi, T., and M. Kawano: Measurement of infrared dichroism for the crystal of lauric acid in the CsBr region. Bull. Chem. Soc. Japan 32, 890 (1959).

196. Shimanouchi, T., S. Tsuchiya, and S. Mizushima: Infrared spectra and molecular structure of polyvinyl chloride. Kobunshi 8, 202-207 (1959).

197. - - - $\mathrm{C}-\mathrm{Cl}$ stretching frequencies and the molecular structure of polyvinyl chloride. J. chem. Phys. 30, 1365 - 1366 (1959).

198. Signer, R., und J. WeILER: Raman-Spektrum und Konstitution hochmolekularer Stoffe. Helv. chim. Acta 15, 649-657 (1932).

199. Simanouti, T.: The normal vibrations of polyatomic molecules as calculated by Urey-Bradley field. II. Vibrations of polythene, ethane, and their deuterium compounds. J. chem. Phys. 17, 734-737 (1949).

200. - and S. Mizushima: The constant frequency Raman lines of n-paraffins. J. chem. Phys. 17, 1102-1106 (1949).

201. Simon, A., M. MǗкLICh, D. Kunath, u. G. Heintz: Über raman-and ultrarotspektroskopische Untersuchungen an Hochpolymeren. J. Polymer. Sci. 30. $201-226$ (1958).

202. Slowinski, E. J., JR., H. WaLter, and R. L. Miller: On the determination of methyl content in polyethylenes. J. Polymer Sci. 19, 353--358 (1956).

203. Smith, R. A., F. E. Jones, and R. P. Chasmar: The detection and measurement of infrared radiation. Oxford University Press, 1957.

204. SNYDER, R. G.: Intramolecular and intermolecular coupling of $\mathrm{CH}_{2}$ rocking modes in n-paraffins. J. chem. Phys. 27, 969-970 (1957).

205. - Private communication, 1959.

206. Stein, R. S.: Interaction of methylene deformation frequencies in paraffin crystals. II. A theoretical treatment of the interaction of vibrations. J. chem. Phys. 23, 734-736 (1955).

207. - and G. B. B. M. SUtherLAND: Interaction of the methylene deformation frequencies in paraffin crystals. J. chem. Phys. 21, 370-371 (1953).

208. - - Effect of intermolecular interactions between $\mathrm{CH}$ frequencies on the infrared spectra of n-paraffins and polythene. J. chem. Phys. 22, 1993 1999 (1954).

209. Stepanov, B.: A theory of vibrational spectra of polyatomic molecules. $\mathrm{V}$. Calculation and interpretation of spectra of normal hydrocarbons. Acta physicochim. USSR 22, 238-262 (1947).

210. Stewart, J.E.: Use of partially oriented solid samples and unpolarized radiant energy in the analysis of infrared absorption spectra. J. chem. Phys. 23, 986-987 (1955).

211. Stuart, H. A.: Die Struktur des freien Moleküls. Berlin: Springer-Verlag 1952.

212. Stuart, A. V., and G. B. B. M. Sutherdand: The effect of hydrogen bonding on the hindered rotation of the hydroxyl group in alcohols. J. chem. Phys. 20, 1977 (1956).

212a. - - Effect of hydrogen bonding on the deformation frequencies of the hydroxyl group in alcohols. J. chem. Phys. 24, 559-570 (1956). 
213. SutherLAND, G. B. B. M.: Some problems in the interpretation of the infrared spectra of large molecules. Disc. Faraday Soc. 9, 274-281 (1950).

214. - Infrared analysis of the structure of amino acids, polypeptides and proteins. Advanc. Protein. Chem. 7, 291-318 (1952).

215. - and D. M. Dennison: The potential functions of polyatomic molecules. Proc. roy. Soc. A 148, 250-271 (1935).

215a. TADOKORO, H.: Infrared studies of polyvinyl alcohol by deuteration of its $\mathrm{OH}$ groups. Bull. Chem. Soc. Japan 32, 1252-1257 (1959).

215b. - Crystallization sensitive band of polyvinyl alcohol. Bull. Chem. Soc. Japan $32,1334-1339$ (1959).

216. TADokoro, H., S. SEKI, and I. Nitra: The crystallinity of solid high polymers. I. The crystallinity of polyvinyl alcohol film. Bull. chem. Soc. Japan 28, $559-564(1955)$.

217. - - - Infrared absorption spectrum of deuterated polyvinyl alcohol film. J. chem. Phys. 23, 1351-1352 (1955).

218. - - - Some information on the infrared absorption spectrum of polyvinyl alcohol from deuteration and pleochroism. J. Polymer Sci. 22, 563-566 (1956).

219. - K. KôzaI, S. SEKI, and I. NitTa: On the crystalline band in the infrared absorption spectrum of polyvinyl alcohol. J. Polymer Sci. 26, 379-382 (1957).

220. - S. Seki, I. Nitta, and R. Yamadera: X-ray and infrared-microspectroscopic measurements on a highly double-oriented specimen of polyvinyl alcohol. J. Polymer Sci. 28, 244-247 (1958).

221. - N. Nishiyama, S. Nozakura, and S. Murahashi: Infrared spectra of isotactic polystyrene and isotactic poly- $\alpha, \beta, \beta$-trideuterostyrene. J. Polymer Sci. 36, 553-556 (1959).

222. - S. Nozakura, T. Kirazawa, Y. Yasuhara, and S. Murahashi: Infrared absorption bands possibly associated with the helical structure of polystyrene, poly-p-methylstyrene, and poly-m-methylstyrene prepared with Ziegler catalyst. Bull. chem. Soc. Japan 32, 313-314 (1959).

222a. TAKEDA, M., K. IIMURA, A. YAMADA, and Y. IMAMURA: Infrared spectra of crystalline and amorphous polystyrene. Bull. chem. Soc. Japan 32, 1151-1153 (1959).

223. Tanaka, C., K. Kuratani, and S. Mizushima: In-plane normal vibrations of methanol. Spectrochim. Acta 9, 265-269 (1957).

224. TARTe, P., and R. Deponthiere: Assignment of the $\mathrm{OH}$ bending frequency in alcohols. J. chem. Phys. 26, 962 (1957).

225. Teller, E.: Theorie der langwelligen Molekülspektren. Hand- u. Jahrb. chem. Physik 9 (II), 43-160 (1934).

226. Theimer, O. H.: Raman and infrared intensities in the vibrational spectra of hydrocarbons. I. Skeletal vibrations of straight zigzag chains. J. chem. Phys. 27, 408-416 (1957).

227. - Raman and infrared intensities in the vibrational spectra of hydrocarbons. II. The $\mathrm{CH}$ vibrations of straight n-paraffins. J. chem. Phys. 27, 1041-1048 (1957).

228. Thompson, H. W., and P. Torkington: The infrared spectra of compounds of high molecular weight. Trans. Faraday Soc. 41, 246-260 (1945).

229. ToBIN, M. C.: The infrared spectra of polymers. II. The infrared spectra of polyethylene terephthalate. J. phys. Chem. 61, 1392-1400 (1957).

230. - Selection rules for normal modes of chain molecules. J. chem. Phys. 23, 891-896 (1955).

231. - A simple crystal powder source for Raman spectroscopy. J. opt. Soc. Amer. 49, 850-855 (1959). 
232. Tobin, M. C., and M. J. Carrano: Infrared spectra of polymers. I. Effect of crystallinity on the infrared spectrum of polyethylene and on the infrared spectra of nylon 6 and nylon 11. J. chem. Phys. 25, 1044-1052 (1956).

233. TschamLer, H.: Frequency assignments for normal aliphatic compounds: $I$. The normal paraffins in the range $1450 \mathrm{~cm}^{-1}$ to $650 \mathrm{~cm}^{-1}$. J. chem. Phys. 22, 1845-1854 (1954).

234. Urey, H. C., and C. A. Bradley: The vibrations of pentatomic tetrahedral molecules. Phys. Rev. 38, 1969-1978 (1931).

235. Walnut, T. H.: A study of selection rules for vibrational spectra of complex crystals. J. chem. Phys. 20, 58-62 (1952).

236. WALTER, E. R., and F. P. REDING : Variations in unit cell dimensions in polyethylene. J. polymer Sci. 21, 561-562 (1956).

237. WARD, I. M.: Rotational isomerism in polyethylene terephthalate. Chem. and Ind. $905-906$ (1956).

238. - Configurational changes in polyethylene terephthalate. Chem. and Ind. 1102 (1957).

239. WARD, J. C.: Measurements on ultraviolet dichroism. Proc. roy. Soc. A 228, 205-219 (1955).

240. Whitcomb, S. E., H. H. Nielsen, and L. H. Thomas: Normal vibrations of chains of similar and similarly situated dynamical systems and the infrared spectrum of undecane. J. chem. Phys. 8, 143-152 (1940).

241. Wiener, O.: Formdoppelbrechung bei Absorption. Kolloidchem. Beih. 23, 189-198 (1926).

242. Wigner, E. P.: Group theory. New York: Academic Press, 1958.

243. Willbourn, A. H.: Polymethylene and the structure of polyethylene: study of short-chain branching, its nature and effects. J. Polymer Sci. 34, 569-597 (1959).

244. WILmshurst, J. K. : Sensitive vibrational frequencies. Part 2. The methylene and methine frequencies and their relation to electronegativity. Canad. J. Chem. 35, 937-941 (1957).

245. Wilson, E. B., JR., J. C. Decius, and P. C. Cross: Molecular vibrations. New York: McGraw-Hill Book Co., 1955.

246. Winston, H., and R. S. HALFORD : Motions of molecular in condensed systems. $V$. Classification of motions and selection rules for spectra according to space symmetry. J. chem. Phys. 17, 607-616 (1949).

247. Wood, D. L.: Small sample infrared spectrophotometry. Rev. Sci. Instr. 26, 787 (1955).

248. - Infrared spectrophotometry, in Methods in enzymology.Vol. IV, pp. 104-126. New York: Academic Press, 1957.

249. - and S. S. Mitra: Effect of convergence on the infrared spectra of anisotropic substances. J. opt. Soc. Amer. 48, 537-542 (1958).

250. WRIGHT, N. : A transmitting polarizer for infrared radiation. J. opt. Soc. Amer. $38,69(1948)$.

251. Yamaguchi, A., I. Ichishima, and S. Mizushima: Polarization of reflected infrared radiation from an echelette grating. Spectrochim. Acta 12, 294—298 (1958).

252. Yoshinaga, H., S. Fujita, S. Minami, A. Mirsuishi, R. A. Oetjen, and Y. YAMADA: Far infrared spectrograph for use from the prism spectral region to about 1-mm wavelength. J. opt. Soc. Amer. 48, 315-323 (1958).

253. Zietlow, J. P., F. F. Cleveland, and A. G. Metster: Substituted methanes. III. Raman spectra, assignments, and force constants for some trichloromethanes. J. chem. Phys. 18, 1076-1080 (1950). 